Annika Wilmers,

Carolin Anda, Carolin Keller, Marc Rittberger (Hrsg.)

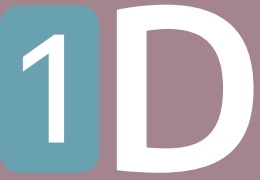

Digitalisierung in der Bildung

\title{
Bildung im digitalen Wandel
}

Die Bedeutung für das pädagogische Personal und für die Aus- und Fortbildung 


\section{Digitalisierung in der Bildung}

Forschungsstand und -perspektiven

herausgegeben von

Michael Kerres, Sybille Stöbe-Blossey,

Ulrike Creß, Marc Rittberger, Josef Schrader

Band 1 
Annika Wilmers, Carolin Anda,

Carolin Keller, Marc Rittberger (Hrsg.)

\section{Bildung im digitalen Wandel}

Die Bedeutung für das pädagogische Personal und für die Aus- und Fortbildung

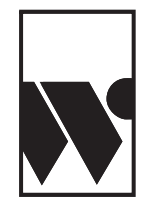

Waxmann 2020

Münster • New York 
GEFÖRDERT VOM

Bundesministerium

für Bildung

und Forschung
Dieses Projekt wird aus Mitteln des Bundesministeriums für Bildung und Forschung unter dem Förderkennzeichen 01JD1800B gefördert. Die Verantwortung für den Inhalt dieser Veröffentlichung liegt bei den Autorinnen und Autoren.

Bibliografische Informationen der Deutschen Nationalbibliothek Die Deutsche Nationalbibliothek verzeichnet diese Publikation in der Deutschen Nationalbibliografie; detaillierte bibliografische Daten sind im Internet über http://dnb.dnb.de abrufbar.

Digitalisierung in der Bildung, Band 1

Print-ISBN 978-3-8309-4199-6

E-Book-ISBN 978-3-8309-9199-1

doi: https://doi.org/10.31244/9783830991991

(C) Waxmann Verlag GmbH, Münster 2020

www.waxmann.com

info@waxmann.com

Umschlaggestaltung: Anne Breitenbach, Münster

Satz: Roger Stoddart, Münster

CC BY-SA 4.0

This work is licensed under a Creative Commons Attribution-ShareAlike 4.0 International License.

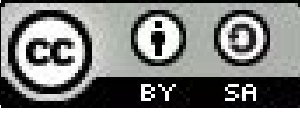




\section{Inhalt}

Annika Wilmers, Carolin Anda, Carolin Keller, Michael Kerres \& Barbara Getto

Reviews zur Bildung im digitalen Wandel:

Eine Einführung in Kontext und Methodik

Iris Nieding \& E. Katharina Klaudy

Digitalisierung in der frühen Bildung

Der Umgang mit digitalen Medien im Spannungsfeld

zwischen Schutzraum und Schlüsselkompetenz

Bettina Waffner

Unterrichtspraktiken, Erfahrungen und Einstellungen

von Lehrpersonen zu digitalen Medien in der Schule. .57

Marcel Capparozza \& Gabriele Irle

Lehrerausbildende als Akteure für die Digitalisierung

in der Lehrerbildung: Ein Review

Katharina Hähn \& Monique Ratermann-Busse

Digitale Medien in der Berufsbildung -

Eine Herausforderung für Lehrkräfte und Ausbildungspersonal?

.129

Jan Koschorreck \& Angelika Gundermann

Die Implikationen der Digitalisierung für das Lehrpersonal

in der Erwachsenen- und Weiterbildung

Ein Review ausgewählter empirischer Ergebnisse und weiterer

theoriebildender Literatur. .159 

Annika Wilmers, Carolin Anda, Carolin Keller,

Michael Kerres \& Barbara Getto

\title{
Reviews zur Bildung im digitalen Wandel: Eine Einführung in Kontext und Methodik
}

\begin{abstract}
Bei der Anwendung von digitalen Medien und der Wissensvermittlung über digitale Technologien kommt dem pädagogischen Personal aller Bildungsbereiche eine Schlüsselposition zu. Die Frage nach der Rolle des pädagogischen Personals in diesem Prozess berührt unterschiedliche Aspekte von der Haltung und Einstellung der Lehrenden über ihre Kompetenzen hin zu allgemeinen Entwicklungen in den Bildungsbereichen sowie zu Maßnahmen, die zur Aus- und Fortbildung von Lehrenden umgesetzt werden. Dieser Frage und ihren Unterthemen wird im vorliegenden Sammelband durch ein Reviewformat nachgegangen, das sich am Verfahren von Critical Reviews orientiert und entsprechend den von Grant und Booth festgelegten Kriterien (Grant \& Booth, 2009) die wichtigste Literatur im Feld identifiziert, die Qualität der recherchierten Literatur systematisch erfasst und in Anlehnung an den jeweiligen Fokus der Fragestellung auswertet. Das Kapitel bietet zunächst eine allgemeine Einführung in den bildungspolitischen und forschungsbezogenen Kontext des Themas. Danach werden die Strategie der systematischen Literatursuche und die Kodierung erläutert. Die Literatursuche erfolgte für den Zeitraum Januar 2010 bis Mai 2019 mit festgelegten deutschen und englischen Schlagwörtern in den Datenbanken des Fachportals Pädagogik (FIS Bildung Literaturdatenbank), ERIC, DNB und Google Scholar.

Schlüsselwörter: Reviewverfahren, Critical Review, Methodik, Einführung, Pädagogisches Personal, Digitalisierung
\end{abstract}

\section{Reviews of education in an era of digital change: An introduction to context and methodology}

Pedagogical staff across all educational sectors play a key role in implementing digital devices and in transferring knowledge with the help of digital technologies. Questions concerning the role of pedagogical staff in this process affect different aspects which range from the attitudes and mind-sets of teachers and instructors to their competences and to general developments in the educational fields as well as measures taken to enhance the teachers' education and training. This anthology presents reviews focusing on such questions, which are based on the method of a critical review. According to the criteria set by Grant and Booth (Grant \& Booth, 2009), the reviews identify the relevant literature, capture the quality of researched literature systematically and evaluate the findings with a focus on the research questions. First, this chapter presents a general introduction to the education policy and the research-oriented context of the topic. Thereafter, strategies of a systematic literature search and the coding process are explained. The literature retrieval was run in the databases Fachportal Pädagogik (Education Research Portal), ERIC, DNB

Bildung im digitalen Wandel

Die Bedeutung für das pädagogische Personal und für die Aus- und Fortbildung

doi: https://doi.org/10.31244/9783830991991.01 
(German National Library) and Google Scholar with pre-defined German and English search terms for the period January 2010 until May 2019.

Keywords: review process, critical review, method, introduction, pedagogical staff, digitisation

\section{Einführung in den Reviewband zur Bedeutung der Digitalisierung für das pädagogische Personal}

Das pädagogische Personal - hier verstanden als Oberbegriff für Pädagoginnen und Pädagogen, Erzieherinnen und Erzieher, Lehrkräfte sowie Lehrerausbildende, die in den verschiedensten Bildungsstufen von der Frühen Bildung bis zur Erwachsenenbildung tätig sind - stellt einen zentralen Akteur im Prozess der Digitalisierung im Bildungswesen dar. Ohne Frage sind die Herausforderungen an diese Berufsgruppe in allen Bildungsbereichen mit Blick auf Digitalisierung vielfältig, kommt ihr doch eine Schlüsselposition bei der Anwendung von digitalen Medien und Vermittlung von Wissen über digitale Medien zu. Die Frage nach der Rolle des pädagogischen Personals in diesem Prozess berührt ganz unterschiedliche Aspekte von der Haltung und Einstellung der Lehrenden über ihre Kompetenzen hin zu allgemeinen Entwicklungen auf dem Gebiet sowie zu Maßnahmen, die zur Ausund Fortbildung von Lehrkräften umgesetzt werden. Mit der strategischen Kompetenzentwicklung von Lehrenden und Lernenden beschäftigen sich entsprechend verschiedene politische Papiere der EU und der Bundesregierung. So definierte die Europäische Kommission 2018 digitale Kompetenz als souveräne und kritische Nutzung digitaler Technologien. Diese Nutzung „umfasst die Kenntnisse, Fertigkeiten und Einstellungen, die alle Bürgerinnen und Bürger in einer sich rasant veränderten Gesellschaft brauchen“ (Europäische Kommission, 2018, S. 8). Die Auseinandersetzung mit Digitalität wird als ein lebenslanger Prozess verstanden: „Der Erwerb digitaler Kompetenzen muss im frühen Kindesalter beginnen und ein Leben lang fortgesetzt werden“ (ebd., S. 9).

Der vorliegende Sammelband fragt nach der Bedeutung der Digitalisierung für das pädagogische Personal und für dessen Aus- und Fortbildung und möchte damit der bedeutenden Rolle dieser Gruppe für die Umsetzung von Digitalisierung in den verschiedenen Bildungsinstitutionen Rechnung tragen. Wie ist die Einstellung von Erziehenden und Lehrkräften zur Digitalisierung, welchen Einfluss hat diese Entwicklung auf ihre fachlichen Kompetenzen und welche Auswirkungen haben die zunehmende Digitalisierung und die Umsetzung digitaler Maßnahmen auf ihre Aus- und Fortbildung? Dieser Sammelband ist der erste Teil einer mehrteiligen Reviewserie zur Digitalisierung in der Bildung, deren Anliegen es ist, aktuelles Forschungswissen $\mathrm{zu}$ verschiedenen inhaltlichen Fragestellungen der digitalen Bildung zu synthetisieren und zu strukturieren. Folglich reflektieren die Reviews den 
Forschungsstand, fassen wesentliche Erkenntnisse zusammen und identifizieren offene Forschungsfragen sowie unerforschte Themenfelder.

Die Reviews entstehen als Teil des Metavorhabens „Digitalisierung im Bildungsbereich" (Digi-EBF), welches innerhalb des BMBF Rahmenprogramms empirische Bildungsforschung (Rahmenprogramm II) Förderprojekte in der Förderlinie „Digitalisierung im Bildungsbereich - Grundsatzfragen und Gelingensbedingungen“ begleitet. $\mathrm{Zu}$ diesem Zweck setzt es eigenständige Forschungsvorhaben aus einer übergeordneten Perspektive um, führt unterstützende Maßnahmen durch, beispielsweise zur Vernetzung der Projekte, und bietet Veranstaltungen zum Fachaustausch und Wissenstransfer an. ${ }^{1}$ Im Reviewprozess kooperieren das DIPF | Leibniz-Institut für Bildungsforschung und Bildungsinformation, an welchem die Recherche und Koordination des Projekts angesiedelt ist, und die Universität Duisburg-Essen (UDE), das Deutsche Institut für Erwachsenenbildung - Leibniz-Zentrum für Lebenslanges Lernen (DIE) und das Leibniz-Institut für Wissensmedien (IWM), die die jeweilige fachspezifische Kompetenz stellen. Die inhaltliche Aufschlüsselung der Bildungsbereiche erfolgt in die Sektoren Bildung in Kindheit, Jugend und Familie, Schulische Bildung, Berufliche Bildung, Lehrerbildung und Erwachsenenbildung. Damit spiegelt die Struktur die Zuordnung der geförderten Projekte zu den entsprechenden Bildungsbereichen wider. ${ }^{2}$

Im Folgenden wird zunächst der bildungspolitische und forschungsbezogene Kontext des Themas aufgezeigt. Danach wird die Methodik erläutert, die diesem dezentral angelegten Reviewverfahren zu Grunde liegt, bevor abschließend in die einzelnen Kapitel aus den Bildungssektoren eingeführt wird.

\section{Bildungspolitischer und forschungsbezogener Kontext}

\subsection{Digitale Kompetenzen des pädagogischen Personals}

Seit über 10 Jahren werden die Auswirkungen, Herausforderungen und Chancen der Digitalisierung in der Bildung auf allen politischen Ebenen, der europäischen, der Bundes- und der Landesebene, diskutiert. Auf europäischer Ebene finden sich Vorschläge zum allgemeinen Themenfeld Digitale Gesellschaft in der Strategie Europa 2020. Diese trat im Juni 2010 in Kraft und zielt auf ein nachhaltiges und integratives Wachstum der jeweiligen Wirtschaftssysteme der Mitgliedsstaaten ab. Die Strategie gründet sich auf sieben Flaggschiffinitiativen, wovon drei Initiativen explizit bildungspolitische Handlungsfelder beschreiben, sich jedoch nur eine mit

1 Vgl. zu Digi-EBF und den aktuellen Projekten im BMBF-Rahmenprogramm EBF, „Digitalisierung im Bildungsbereich“ https://digi-ebf.de/ und https://www.empirische-bildungsforschung-bmbf.de/ de/2563.php (zuletzt aufgerufen am 30.01.2020).

2 Der Hochschulbereich ist hingegen in der Förderlinie „Digitale Hochschulbildung" angesiedelt und hier nicht vertreten. https://www.bmbf.de/de/digitale-hochschullehre-2417.html (zuletzt aufgerufen am 27.01.2020) 
dem Thema Digitalisierung befasst. Eine in der Strategie Europa 2020 spezifizierte Digitale Agenda flankiert die Initiativen durch den Ausbau des Breitband-Internets, die Förderung eines gemeinsamen Marktes für internetbezogene Dienstleistungen und schnellere Netzzugänge. Daneben fokussiert die Initiative Jugend in Bewegung die Verbesserung der Bildungssysteme und die Förderung der internationalen Attraktivität der höheren Bildung in Europa. Die Agenda für neue Fähigkeiten und Jobs zielt auf eine Modernisierung des Arbeitsmarkts durch die Förderung von Arbeitsmobilität und lebenslangem Lernen ab, um die Arbeitsnachfrage und -angebote besser aufeinander abstimmen zu können.

Während diese Initiativen also vor allem um technologische und wirtschaftliche Herausforderungen kreisen, befasst sich der EU-Aktionsplan Digitale Bildung aus dem Jahr 2018 des Rats für Bildung, Jugend, Kultur und Sport mit konkreteren inhaltlichen Zielen. Diese sollen im Zuge der Digitalisierung eine kontinuierliche Verbesserung der Bildung in der Schul-, Berufs- und Hochschulbildung herbeiführen. Der Aktionsplan stellt die bessere Nutzung digitaler Technologien im Unterricht und zu Lernzwecken, die Entwicklung relevanter digitaler Fertigkeiten und Kompetenzen für den digitalen Wandel sowie eine qualitativ hochwertige Bildung durch aussagekräftigere Datenanalysen und Prognosen heraus (EU Kommission, 2018, S. 5). Der Aktionsplan setzt des Weiteren auf eine stärkere Vernetzung der pädagogischen Fachkräfte durch Programme wie Erasmus+ und eigens dafür eingerichtete digitale Plattformen und das Messinstrument SELFIE ${ }^{3}$ zur Selbsteinschätzung der digitalen Fähigkeiten. Die jüngst veröffentlichte EU-Strategie für Daten sieht eine Überarbeitung des EU-Aktionsplans Digitale Bildung hinsichtlich einer verbesserten Datennutzung und Erhebung durch Learning Analytics vor (Europäische Kommission, 2020, S. 22).

Bildungssektoren übergreifend wird auch die Verbesserung digitaler Kompetenzen von Lernenden und Lehrenden angestrebt. Der EU-Aktionsplan Digitale Bildung benennt fünf digitale Kompetenzbereiche: (1) Informations- und Datenkompetenz, (2) Kommunikation und Zusammenarbeit, (3) Erstellung digitaler Inhalte, (4) Sicherheit und Wohlergehen und (5) Problemlösung (Europäische Kommission, 2018, S. 8). Anknüpfend an die Kompetenzmodelle für lebenslanges Lernen aller EU-Bürger und Bürgerinnen aus der Agenda 2020 wurde speziell für Lehrkräfte ein Referenzrahmen für digitale Kompetenzen $(D i g C o m p E d u)^{4}$ entwickelt, der auf die systematische Förderung digitaler Kompetenzen von Lehrenden abzielt. Das Modell umfasst sechs Bereiche und beschreibt 22 Kompetenzen. Für die Umsetzung des Modells werden die kontinuierliche Fort- und Weiterbildung der Lehrkräfte fokussiert sowie die Verankerung digitaler Kompetenzen in deren Ausbildung (Redecker \& Punie, 2017). Abbildung 1 zeigt das Modell und die jeweiligen Kompetenzbereiche.

3 https://ec.europa.eu/education/schools-go-digital_de (zuletzt aufgerufen am 20.01.2020)

4 https://ec.europa.eu/jrc/en/digcompedu (zuletzt aufgerufen am 20.01.2020) 


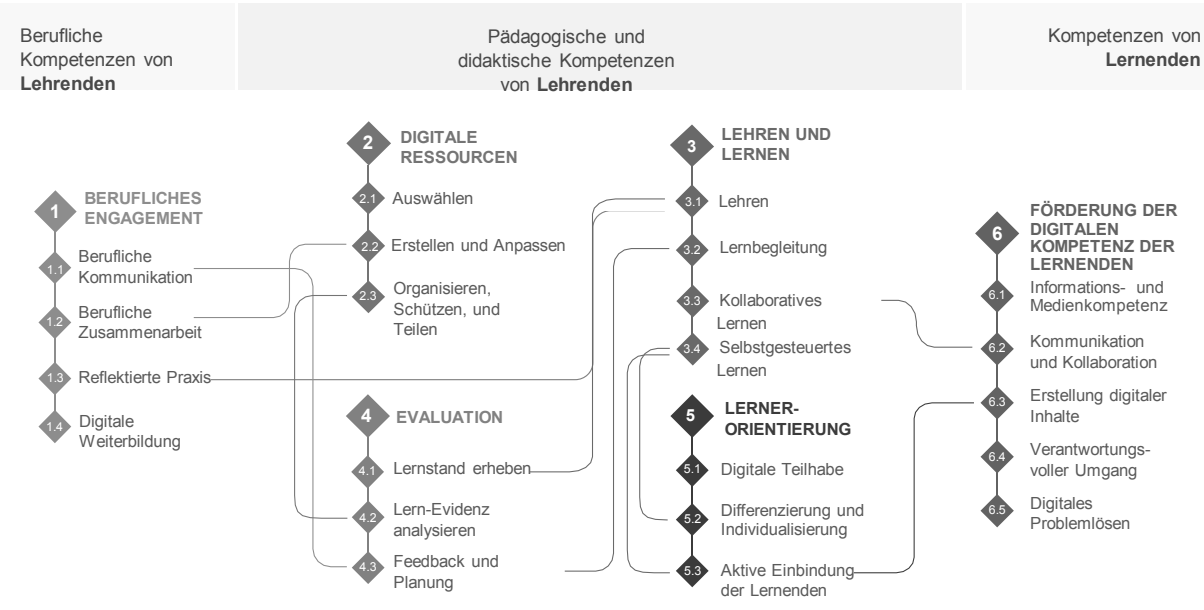

Abbildung 1: Europäisches Kompetenzmodell für Lehrkräfte und Lehrende DigCompEdu Quelle: Redecker \& Punie, 2017, S. 8, dt. Übersetzung Goethe-Institut

Ähnlich wie die Agenden und Aktionspläne der EU-Kommission entwickelte die Bundesregierung ihre Strategie Bildungsoffensive für die digitale Wissensgesellschaft als Erweiterung ihrer Digitalen Agenda 2014-2017 im Jahr 2016. Die Strategie stellt einen „systematischen Handlungsrahmen dar, um die digitale Transformation des Bildungssystems voranzutreiben" (BMBF, 2016, S. 5). Sie fokussiert eine strategische Organisationsentwicklung mit digitaler Bildungsverwaltung, eine verbesserte bildungspolitische Steuerung, den Einsatz von Learning Analytics und ein zukunftsfähiges Bildungsmanagement. Die allgemeine Datensicherung und die Datensicherheit bilden innerhalb der Strategie ebenfalls wichtige Bestandteile. Alle Bildungsbereiche werden dabei in den Blick genommen, jedoch sind für jeden Bereich eigene Maßnahmen angesetzt. Der Schutz der Daten soll durch die Schaffung von Rechtsrahmen erzielt werden, die die Produktion und die Nutzung digitaler Bildungsangebote regeln und das Urheberrecht achten. Die Umsetzung der Strategie erfolgt durch Kooperationen zwischen Bund und Ländern und mit Hilfe länderübergreifender Förderprogramme.

In der Schulpolitik war bisher die Stärkung der digitalen Infrastruktur und der technischen Ausstattung prioritär (Blossfeld et al., 2018; Burow, 2019; Deutscher Bundestag, 2016). Die Umsetzung erfolgte durch den Digitalpakt\#D, der 2018 beschlossen wurde und im Jahr 2019 mit fünf Milliarden Euro in Kraft trat (BMBF, 2016, S. 22). Um die Potenziale der angehenden Lehrkräfte zu stärken, wurde die Qualitätsoffensive Lehrerbildung 5 initiiert. Diese bildet Synergien mit der Strategie der Kultusministerkonferenz (KMK) Bildung in der digitalen Welt ${ }^{6}$ und dem Kompetenzrahmen DigCompEdu der EU. Die Qualitätsoffensive hat zum Ziel, die me-

5 https://www.qualitaetsoffensive-lehrerbildung.de/ (zuletzt aufgerufen am 27.01.2020)

6 https://www.kmk.org/fileadmin/Dateien/veroeffentlichungen_beschluesse/2018/Strategie_Bildung_ in_der_digitalen_Welt_idF._vom_07.12.2017.pdf (zuletzt aufgerufen am 22.01.2020) 
dienpädagogischen Kompetenzen von Lehrenden und Lernenden zu stärken und die Anforderungen einer digitalen Welt in die Aus- und Fortbildung von Lehrkräften zu integrieren. Dies sieht im Rahmen der digitalen Hochschulbildung zudem die Anpassung der Curricula der beruflichen und akademischen Bildung sowie eine Modernisierung der Ausbildungsordnungen und Studiengänge vor (KMK, 2017, S. 46-53).

Auch über die Sektoren Schule und der Lehrerbildung hinaus finden strategische Umsetzungen für den Sektor berufliche Bildung und Weiterbildung statt. Im Rahmen der Bildungsoffensive für die digitale Wissensgesellschaft wurde 2016 die Dachinitiative Berufsbildung $4.0^{7}$ gegründet, um die berufliche Aus- und Weiterbildung auf die Anforderungen einer digitalen Arbeitswelt auszurichten. Dies soll durch die Verankerung digitaler Medien in der beruflichen Bildung und durch die Schaffung von Strukturen für die Implementierung digitalen Lernens in die Ausbildung erreicht werden. Die benötigten Kompetenzen der Facharbeitenden und Ausbildenden sollen dabei durch passende Qualifizierungsangebote gefördert werden. So unterstützt z.B. das Programm Jobstarterplus die Entwicklung von kleinen und mittelständigen Unternehmen, um die personellen Anforderungen, die im Zuge von Automatisierung und Digitalisierung eintreten, zu meistern. Die Qualifizierungsinitiative Digitaler Wandel - Q $4.0^{8}$ setzt des Weiteren auf Weiterbildungskonzepte, mit denen Ausbildende passgenaue Medien- und IT-Kompetenzen erwerben können. Ebenso wird der Ausbau der Infrastruktur überbetrieblicher Berufsbildungsstätten (ÜBS) und Kompetenzzentren durch das Sonderprogramm ÜBS-Digitalisierung bis 2023 vorangetrieben, um eine modernisierte Ausbildung für Fachkräfte zu gewährleisten.

Für den Bereich frühkindliche Bildung sieht die BMBF Strategie Bildungsoffensive für die digitale Wissensgesellschaft (BMBF, 2016, S. 15) vor, die Verbreitung handlungsorientierten Fachwissens für Elementarpädagoginnen und -pädagogen durch Weiterbildungsinitiativen (WiFF) zu unterstützen. In der Fachkräfteoffensive Erzieherinnen/Erzieher des Bundesministeriums für Familie, Senioren, Frauen und Jugend von 2019 ist die praxisnahe Anleitung und Qualifizierung der Auszubildenden ein wichtiger Bestandteil, um die Qualität in der frühen Bildung zu gewährleisten und den Fachkräftemangel einzudämmen. ${ }^{9}$ Eine explizite Ausführung zur Digitalisierung mit Blick auf die Kompetenzen des pädagogischen Personals im Bereich der frühkindlichen Bildung wird darin allerdings nicht getroffen.

Es lässt sich festhalten, dass bestimmte Anforderungen, wie z.B. technische Infrastrukturen und Medien- und IT-Kompetenzen, die im Zuge der Digitalisierung auf die Sektoren des Bildungssystems zukommen, auf den politischen Ebenen erkannt wurden. Dennoch kann trotz einiger konkreter Modelle und Maßnahmen

7 https://www.bmbf.de/upload_filestore/pub/Berufsbildung_4.0.pdf (zuletzt aufgerufen am 22.01.2020)

8 https://www.bmbf.de/de/qualifizierungsinitiative-digitaler-wandel---q-4-0-10065.html (zuletzt aufgerufen am 22.01.2020)

9 https://www.bmfsfi.de/blob/131404/18d38040fe0b1661dc0550d1db189349/fachkraefteoffensiveerzieherinnen-erieher-giffey-data.pdf (zuletzt aufgerufen am 22.01.2020) 
eine systematische Verankerung von Qualifizierungsmaßnahmen für das pädagogische Personal noch nicht für alle Bildungssektoren beschrieben werden. Die EU hat die Förderung einer solchen Verankerung für das nächste europäische Rahmenprogramm Horizont Europa ${ }^{10}$ von 2021-2027 angekündigt. Die technische Ausstattung und Modernisierung der Bildungsinstitutionen sowie zukunftstaugliche Aus- und Weiterbildungsprogramme bilden eine Grundlage, deren erfolgreiche Umsetzung sich erst in den kommenden Jahren zeigen wird. Eine enge Zusammenarbeit zwischen der Bildungspraxis und der Bildungsforschung kann hier unterstützend wirken. Das BMBF-Rahmenprogramm Empirische Bildungsforschung, und dessen Forschungsschwerpunkt Digitalisierung im Bildungsbereich, in dessen Rahmen der vorliegende Sammelband entstanden ist, fokussiert erstens eine praxisnahe Forschung und lösungsorientierte Ansätze durch die Förderung der Grundlagenforschung zu aktuellen Fragestellungen zur Digitalisierung und zweitens eine Schwerpunktsetzung auf den Transfer von Forschungsergebnissen in Politik, Bildungspraxis und Gesellschaft. Das im Folgenden vorgestellte Reviewverfahren bewegt sich an der Schnittstelle beider Anliegen.

\subsection{Zur Rolle von Reviews zur Digitalisierung in der Bildungsforschung}

Im Rahmen des BMBF-Metavorhabens Digitalisierung im Bildungsbereich (Digi$E B F$ ) kommt der Auswertung und Zusammenführung vorliegender Ergebnisse - sei es aus BMBF-geförderten Projekten oder anderen Vorhaben - eine große Bedeutung zu. Der Stand der Forschung zu zentralen Fragen des digitalen Lernens wird in einer Serie von Reviews erarbeitet, über verschiedene Portale ${ }^{11}$ sektorenspezifisch kommuniziert und laufenden Fragestellungen und Ergebnissen der Förderprojekte gegenübergestellt.

Die Anzahl der publizierten Befunde aus den unterschiedlich angelegten Entwicklungs- und Forschungsprojekten steigt und damit auch die Unübersichtlichkeit über die Befundlage. In vielen Vorhaben wird den Potenzialen der Digitalisierung für die Bildung nachgegangen. Dabei beschäftigt sich die Forschung mit ganz unterschiedlichen Bildungsinstitutionen und -kontexten entlang des lebenslangen Lernens. Gängige Fragen wären beispielsweise: Wie ist der Stand der Bildungsforschung zum Lernen mit digitalen Medien? Welche grundlegenden Erkenntnisse liegen zum Lernen mit digitalen Medien vor? Welche Annahmen lassen sich klar belegen oder widerlegen? Welche Fragen sind weiterhin offen und sind weiter zu bearbeiten? Welchen Einfluss hat der Bildungskontext und gibt es Unterschiede in Abhängigkeit von der institutionellen Rahmung und individuellen Einflussfaktoren?

10 https:/ec.europa.eu/info/horizon-europe-next-research-and-innovation-framework-programme_ de (zuletzt aufgerufen am 22.01.2020)

11 Wie bspw. e-teaching.org für den Hochschulbereich, bildungsserver.de für alle Bildungsbereiche oder wb-web.de für den Weiterbildungsbereich. 
Gibt es Abweichungen in der frühen Bildung, beim Lernen in allgemein- und berufsbildenden Schulen ebenso wie in anderen, außerschulischen Kontexten, in der Hochschule, der Erwachsenenbildung, der beruflichen Aus- und Weiterbildung sowie der weiteren betrieblichen Bildungsarbeit, bis hin zum Lernen in der Freizeit, in non-formalen oder gar informellen Kontexten? Die Digitalisierung hat nicht nur für das Lernen und für die Arbeit in bestehenden Bildungsinstitutionen Bedeutung, sondern es entwickeln sich neue Lernorte und -möglichkeiten jenseits traditioneller Strukturen. Da die Forschung zu diesen Fragen international angelegt ist, bleibt schließlich die Frage, welche kulturellen und politischen Rahmungen sie haben oder ob sie unabhängig hiervon zu rezipieren sind?

Damit wird deutlich, wie komplex Forschungsfragen zur Digitalisierung in der Bildung angelegt sind und warum es wichtig ist, bestehende Forschungsergebnisse zu systematisieren, zu synthetisieren und der weiteren Diskussion zugänglich zu machen. Denn manche Studien zeigen große Vorteile des Einsatzes digitaler Medien beim Lernerfolg, andere belegen keine signifikanten Unterschiede, und wieder andere Untersuchungen verweisen auf geringere Lernerfolge. Mit dem statistischen Verfahren der Metaanalyse, das in der internationalen Forschung zu Educational Technology eine große Bedeutung hat, werden Effektgrößen von Einzelstudien zusammengeführt. Um den Effekt des „Treatments“ vergleichbar zu machen, werden alle weiteren Variablen, die den Zusammenhang beeinflussen könnten, ausgeblendet. Seit der ersten Publikation von Kulik, Kulik und Cohen (1980) werden in diesem Feld seit nunmehr 40 Jahren Metaanalysen durchgeführt: angefangen vom computer based training bis hin zu game based learning, social learning und virtual learning environments. Dabei zeigt sich ein bemerkenswert konstanter Befund, der auch in den mittlerweile durchgeführten Meta-Metaanalysen von bspw. Tamim, Bernard, Borokhovski, Abrami und Schmid (2011) sichtbar wird: Die Studien verweisen übereinstimmend auf einen kleinen bis moderaten Vorteil (vgl. ebd.) des Einsatzes digitaler Medien. Damit lässt sich einerseits konstatieren, dass die Digitalisierung im Ganzen nicht zu schlechteren Lernleistungen beiträgt. Andererseits ist ernüchternd festzuhalten, dass die vielfach behaupteten Durchbrüche und Revolutionen des Lernens durch die Digitalisierung nicht zu erwarten sind.

Gleichzeitig mehren sich die Stimmen, die die Anlage entsprechender Metaanalysen für die Bildungsforschung infrage stellen. Randomisierte kontrollierte Studien mögen zudem für die medizinische Forschung das beste Studiendesign sein, ob dieses Design auf Bildungskontexte allerdings übertragbar ist, wird durchaus kritisch diskutiert. Ist es zielführend, E-Learning und die Digitalisierung als ein Treatment aufzufassen, das den traditionellen Lernformen gegenüberzustellen ist, so wie die Behandlung von Krankheitssymptomen mit einem neuen Medikament, das mit einer Placebo-Gruppe verglichen wird? Aus Sicht der Mediendidaktik wirken digitale Medien und Technologien nicht als solche im Lehr-Lerngeschehen, sondern erst in Handlungen von Lehrenden und Lernenden, die sich immer in gegebenen Situationen und sozialen Kontexten vollziehen (Kerres, 2003). Die situative und 
kulturelle Einbettung ist aus mediendidaktischer Perspektive entscheidend, um die Erfolgsfaktoren und Gelingensbedingungen des Lernens mit digitalen Medien identifizieren zu können: nicht ob das Lernen mit digitalen Medien zum Lernerfolg beiträgt, sondern unter welchen Bedingungen dies möglich wird und welche Faktoren sich hierbei regelmäßig als zuträglich erweisen. Gibt es spezifische Konstellationen des Lernens mit digitalen Medien, die sich im Vergleich zu anderen Settings als vorteilhaft erweisen? Eine angemessene Einordnung entsprechender Befunde bedarf einer mehrdimensionalen Betrachtung von didaktischen Konfigurationen, die sich der einfachen Logik des A oder B entziehen. Hinzu kommt die grundlegende Erkenntnis, dass unterschiedliche didaktische Ansätze und Konzepte grundsätzlich auf unterschiedliche Zieldimensionen einzahlen und insofern schwer zu vergleichen sind: „Direct instruction“ kann die Vermittlung und Wiedererinnerung von Wissen besser unterstützen als „problembasierte Ansätze“, die wiederum eher zum Erwerb von Problemlösekompetenzen, Selbstregulation und Überblickswissen beitragen (Kirschner, Sweller \& Clark, 2006).

Es stellt sich damit die Frage, wie solche komplexeren Befunde und Konstellationen aus Einzelstudien zusammengeführt werden können, ohne sich erneut in der Vielfalt der Befundlage zu verlieren. Reviews sind das klassische Verfahren, um Befunde einzuordnen und verfügbar zu machen. Für die Bildungspraxis sind viele Publikationsorgane schlicht nicht zugänglich und die Rezeption von Einzelstudien kann vielfach nicht erwartet werden. Aus Sicht der Bildungsforschung sind Reviews lange Zeit als reine Zusammenfassung vorliegender Ergebnisse eingeordnet worden, denen ein eigener Erkenntniswert und damit auch eine eigene wissenschaftliche Erkenntnisleistung im Wissenschaftsbetrieb abgesprochen wurde (Newman \& Gough, 2020). Reviews haben sich in den letzten Jahren in ihrer Anlage und in ihrem Anspruch aber deutlich weiterentwickelt. Es hat sich eine eigenständige Methodologie der Review-Verfahren entwickelt, die Befunde von Einzelstudien als Datenmaterial betrachtet. Mithilfe genau beschriebener, dokumentierter und nachvollziehbarer methodischer Arbeitsschritte wird damit ein Beitrag zur Verdichtung von Aussagen oder auch zur Prüfung von Hypothesen geleistet. Auf diese Weise können Reviews eine eigenständige wissenschaftliche Erkenntnisleistung generieren, da sie über die bloße Aggregation hinausgehen.

\section{Zum Verfahren und zur Methodik in diesem Reviewband}

\subsection{Methodische Verortung der Reviews}

Das Spektrum an zur Verfügung stehenden Reviewformaten ist breit gefächert und reicht von weit gefassten Literaturübersichten über Forschungssynthesen, die ihren Schwerpunkt auf bestimmte Arbeitsschritte setzen, bis zu Verfahren mit genau definierter Vorgehensweise beispielsweise für metaanalytische Verfahren (vgl. Grant 
\& Booth, 2009 sowie Fink, 2005). Eine systematische Vorgehensweise ist für jede Form eines Reviews Voraussetzung, gleichwohl liegt einem Systematic Review methodisch die umfassendste Art der Ausarbeitung und Darstellung zugrunde. Für das in diesem Buch dargestellte Vorhaben wurde ein Reviewformat gewählt, das sich am Verfahren für Systematic Reviews orientiert, aber an klar definierten Schnittstellen eine im Vergleich zu Systematic Reviews methodisch abgeschwächte Vorgehensweise aufweist.

Systematic Reviews kennzeichnet ein hohes Maß an Transparenz, ein systematisches, iteratives Vorgehen und eine weitgehend vollständige Recherche sowie eine umfassende Auswertung und Analyse der recherchierten Literatur. Die einzelnen Recherche-, Auswertungs- und Analyseschritte sind auch zu einem späteren Zeitpunkt reproduzierbar und aktualisierbar. Während Systematic Reviews zunächst in der Medizin Anwendung fanden, wird das Verfahren seit dem ausgehenden 20. Jahrhundert auch in andere wissenschaftliche Bereiche wie die Sozialwissenschaften, die Bildungsforschung, die Kriminologie oder die Politikwissenschaft übertragen (vgl. zum Themenfeld aus Perspektive der Bildungsforschung bzw. den Sozialwissenschaften Gough, Oliver \& Thomas, 2017; Petticrew \& Roberts, 2006; Zawacki-Richter, Kerres, Bedenlier, Bond \& Buntins, 2020). Die ersten Institutionen, die sich der Produktion und Bereitstellung von Systematic Reviews in den Sozialwissenschaften, dem Gesundheitssektor und dem Bildungswesen verschrieben haben, waren seit den 1990er Jahren das Evidence for Policy and Practice Information and Co-ordinating Centre in London (EPPI-Centre) und die internationale Campbell Collaboration mit derzeitigem Sitz in Oslo. ${ }^{12}$ Spätestens mit der Ausweitung der Diskussion über den angloamerikanischen Sprachraum hinaus, der Gründung neuerer Educational Clearinghouses in einigen europäischen Ländern ${ }^{13}$ sowie dem Ausbau entsprechender wissenschaftlicher Verfahren, hat die Diskussion um die Methodenwahl für Systematic Reviews zusätzlichen Auftrieb erhalten.

Bei vielen Fragestellungen zeigt sich, dass die Datengrundlage für eine Einengung auf Metaanalyseverfahren oder eine Bevorzugung bestimmter Studiendesigns wie randomisierte, kontrollierte Studien nicht gegeben ist. Das EPPI-Centre hat sich bereits früh dafür ausgesprochen, Systematic-Review-Verfahren als einen methodisch vielfältigen Ansatz zu verstehen (Gough, 2009). Jüngere Literatur betrachtet die Frage nach dem Umgang mit Review-Verfahren zunehmend als einen eigenen Forschungsgegenstand, wie die Studie von Polanin, Maynard und Dell aufzeigt, die im englischsprachigen Raum 25 Synthesen $\mathrm{zu}$ Reviews mit Bildungsthemen identifizierten (Overviews), deren Vorgehensweise verglichen und eine weitere Ausdifferenzierung der Methodik fordern (Polanin et al., 2017; vgl. auch Dixon-Woods et al., 2006; Gough \& Thomas, 2016). Methodenvielfalt zuzulassen und gleichzeitig

12 Die EU hat das Thema des evidenzbasierten Transfers nach der Jahrtausendwende vermehrt auf die Agenda gesetzt. Vgl. hierzu BMBF, 2008; Davies, 2004 u. Oakley et al., 2005. Vgl. auch die Gründung des What Works Clearinghouse in den USA im Jahr 2002.

13 Z. B. das Danish Clearinghouse for Educational Research (2006-2019) oder das Norwegian Knowledge Center for Education als Teil des Research Council Norway (gegründet 2013). 
in der Auswertung der Daten nach transparenten und definierten Kriterien vorzugehen, scheint ein für viele Fragestellungen gangbares Verfahren zu sein. Der Fokus richtet sich dabei gleichermaßen auf eine mögliche Beantwortung von für Bildungspolitik und Bildungspraxis relevanten Fragen wie auf den Verweis von wenig erforschten Themenfeldern und Datenlücken.

Im Folgenden wurde ein Reviewformat gewählt, das im Vergleich zu Systematic Reviews eine komprimiertere Vorgehensweise aufweist. Diese Anpassung ermöglicht es gleichermaßen, über den für das Metavorhaben vorgegebenen Projektzeitraum von fünf Jahren in jährlichem Rhythmus Reviews zu erstellen und an einem systematischen und transparenten Vorgehen nach festgelegten Kriterien für jeden Arbeitsschritt festzuhalten. Die gewählte Methodik orientiert sich an den von Grant und Booth beschriebenen Kriterien für Critical Reviews (Grant \& Booth, 2009, siehe auch Booth, Sutton \& Papaioannou, 2016). Critical Reviews zeichnen sich nach Grant und Booth (2009) durch eine umfangreiche Literatursuche aus, die zur Aufgabe hat, die wichtigste Literatur im Feld zu identifizieren. Die Qualität der recherchierten Literatur wird systematisch erfasst und evaluiert. Allerdings geschieht dies nicht nach einer im Vorfeld festgelegten Methodik, sondern in Anlehnung an den jeweiligen Fokus der Fragestellung. Damit beschreiben Critical Reviews ein eingegrenztes Themengebiet und können zur Theorie- oder Modellbildung im Feld beitragen. Entsprechend konzentriert sich dieses Format inhaltlich vor allem darauf, einen konzeptionellen Beitrag zur Erfassung des Forschungsfeldes zu leisten, während sich Systematic Reviews in der Regel an der Beantwortung einer sehr konkreten Fragestellung orientieren. Mit der gewählten Vorgehensweise lehnt sich das Vorhaben zudem an verkürzte Reviewverfahren an, die auch unter dem Begriff „Rapid Review“ diskutiert werden (Thomas, Newman \& Oliver, 2013). Ein solches Vorgehen weist zwar Abstriche in der Datenauswertung auf, hat gegenüber länger angelegten Verfahren aber den Vorteil, vergleichsweise zügig auf dynamische Forschungsfelder reagieren zu können. Entscheidender Faktor bleibt indes, dass die jeweiligen Stellschrauben im Verfahren transparent und damit aktualisierbar und für spätere Untersuchungen weiter ausbaufähig sind.

\subsection{Suchstrategie}

Im hier zugrunde liegenden Verfahren verständigten sich die Projektmitarbeitenden zunächst auf einen gemeinsamen Rahmen für die Suche (Auswahl der Datenbanken, Festlegung der einbezogenen Sprachen und des maximalen Suchzeitraums insgesamt). Die Ausgestaltung der Suchstrategie erfolgte in Absprache mit den Autorinnen und Autoren der fünf Bildungssektoren und ließ sich so der jeweiligen Ausgangslage, zum Beispiel den sehr unterschiedlich ausfallenden bereits vorhandenen Forschungsständen und fachbezogenen Zielsetzungen anpassen. Im Folgenden 
werden die übergeordneten Suchkriterien sowie sektorspezifische Adaptionen bei der Literatursuche vorgestellt. ${ }^{14}$

Der Rechercheprozess für ein Critical Review zeichnet sich vor allem durch eine umfassende Suche nach Literatur und der Dokumentation der einzelnen Arbeitsschritte aus, um signifikante Forschungsarbeiten zu identifizieren (Booth et al., 2016, S. 2; Grant \& Booth, 2009, S. 93). Dabei beugt eine systematische Herangehensweise Publikations- und Inhaltsverzerrungen (Bias) vor und vereinfacht die Darstellung des Review-Prozess und damit seine Nachvollziehbarkeit. Es sollte vermieden werden, eine umfassende Recherche mit einer sehr ergiebigen Treffermenge bei der Datenbankrecherche gleichzusetzen. Zwar haben alle Forschungssynthesen die Zielsetzung, ein übersichtliches Bild der aktuellen Forschung darzustellen, aber zum einen ist es beispielsweise aufgrund eigener sprachlicher Einschränkungen nicht möglich alle (weltweit) vorhandenen Studien zu einem Themenfeld zu finden (Booth et al., 2016). Ebenso können Studien (noch) nicht in einer Datenbank erfasst sein. Zum anderen kann eine zu hohe Treffermenge bei geringer Präzision den Bias sogar begünstigen, da durch eine situationsbedingte Relevanzbewertung auch viele thematisch entfernte oder qualitativ schlechtere Quellen mit in den Recall einfließen (Booth, 2010, S. 432; Petticrew, 2015, S. 3). Ein qualitativ hochwertiges Review zeichnet sich somit nicht nur durch eine möglichst umfangreiche Recherche aus, sondern vor allem durch eine gezielte und der Forschungsfrage angepassten Literaturrecherche. Darüber hinaus fließen Rahmenbedingungen wie Zeitumfang, Personalkapazitäten und Eingrenzung der Forschungsfrage in die Gestaltung der Recherche mit ein. Es ist daher wichtig, den Suchaufwand ins Verhältnis zu KostenNutzen-Gesichtspunkten zu setzen (Beelmann, 2014, S. 64) und die Gestaltung der Suchstrategie individuell an das Projekt eines Reviews anzupassen.

Die Suchstrategie für das vorliegende Review orientierte sich an der übergreifenden Fragestellung unabhängig der Bildungssektoren und wurde dann in Zusammenarbeit mit den einzelnen Sektoren für deren Zielstellung spezifiziert. Entsprechend beinhaltete dieser mehrstufige Prozess folgende Arbeitsschritte: Zunächst wurde eine Testsuche (Scoping Search) durchgeführt, um Suchterme in den ausgewählten Datenbanken zu überprüfen und festzulegen. Die Recherche erfolgte in den zwei Fachdatenbanken FIS Bildung Literaturdatenbank und ERIC - Education Resources Information Center mit jeweils deutschen und englischen Suchwörtern sowie in einem späteren Schritt in Google Scholar und dem Katalog der Deutschen Nationalbibliothek. Der Zugang zur Datenbank FIS Bildung erfolgte über das Fachportal Pädagogik wodurch zugleich eine Suche in ERIC, der Katalog der Library of Congress, Casalini libri und EBSCOhost ebooks abgedeckt wurde. Die relevante Literatur zu den einzelnen Bereichen erscheint zwar hauptsächlich in den zentralen Fachdatenbanken der Bildungsforschung FIS und ERIC. Jedoch erfassen die-

14 Eine detaillierte Auflistung der Suchabläufe findet sich in der online zugänglichen Dokumentensammlung zum Band: http://dx.doi.org/10.7477/414:1:0. Vgl. zudem die einzelnen Kapitel in diesem Band. 
se Datenbanken nicht alle Sammelwerke oder Zeitschriften, welche zum Beispiel den Bereich der Medienpädagogik abdecken. Durch die Recherche über Google Scholar wurde diese Lücke geschlossen. Der Bibliothekskatalog der Deutschen Nationalbibliothek diente ebenfalls als ergänzende Informationsquelle. Um eine gezielte Recherche durchführen zu können, muss vor allem der Aufbau der Datenbankstruktur bekannt sein und welche Daten darin recherchierbar sind. Nach Durchführung der Testsuchen begannen die umfassende Recherche sowie die erneute Anpassung der Suchstrategie nach Rücksprache mit den Autorinnen und Autoren der Bildungssektoren. Parallel hierzu wurden alle Arbeitsschritte dokumentiert. Eine Herausforderung für die Recherche dieses Reviews war vor allem, eine systematische sowie einheitliche Herangehensweise für die jeweiligen Bildungssektoren zu gewährleisten und dennoch auf ihre besonderen Anforderungen an die Recherche einzugehen.

Aus den inhaltlichen Schwerpunkten der Fragestellung ergaben sich vier Wortgruppen, welche zur Recherche in den Datenbanken kombiniert wurden: (1) Themenschwerpunkt (2) Digitalisierung (3) Bildungssektor (4) Pädagogisches Personal (siehe Abbildung 2).

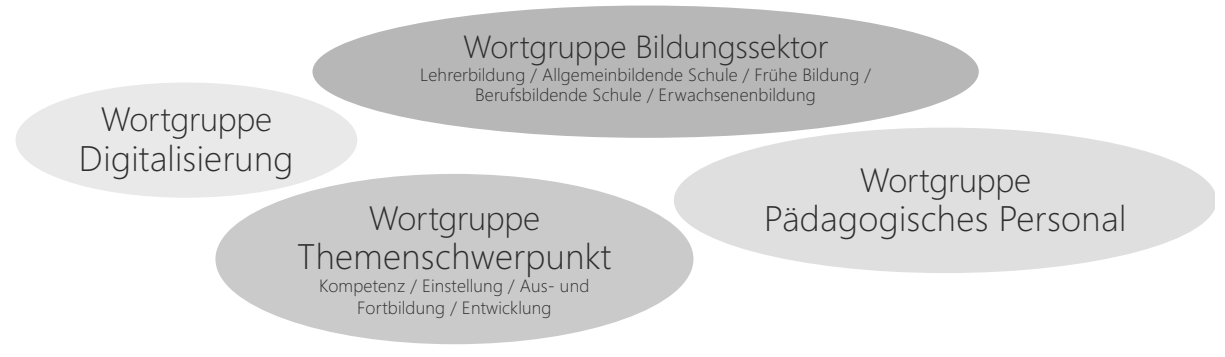

Abbildung 2: Wortgruppen Rechercheschwerpunkte

Zur Differenzierung der Frage nach der Bedeutung der Digitalisierung für das pädagogische Personal wurde die erste Wortgruppe „Themenschwerpunkt“ zudem in vier Bereiche unterteilt, auf welche sich die weitere Recherche fokussierte. Diese sind zum einen (a) die Kompetenz des pädagogischen Personals bezüglich digitaler Technologien, (b) die Einstellung und Haltung des Personals zur Digitalisierung, (c) Aus- und Fortbildung des pädagogischen Personals und (d) aktuelle Entwicklungen zu diesem Thema. Um eine umfassende Recherchestrategie zu diesen Themenblöcken zu entwickeln, wurden passende Suchwörter gesammelt und strukturiert. Hieraus bildete sich ein Pool an Begriffen, welche sich auf die unterschiedlichen Rechercheanfragen für die Themenschwerpunkte und Bildungssektoren übertragen ließen. Als erstes wurden zur zweiten Wortgruppe „Digitalisierung“ sowie zu den vier Themenschwerpunkten der Kompetenz, Einstellung, Aus- und Fortbildung und Entwicklung die wesentlichen Suchwörter identifiziert. Die Suchbegriffe der zu- 
sammengestellten Wortgruppen ließen sich auf die verschiedenen Bildungssektoren übertragen und mussten gegebenenfalls minimal angepasst werden. Da die für dieses Review verwendeten Datenbanken Bildungsforschung allgemein bedienen, wurde die Recherche um eine dritte Wortgruppe erweitert, die den jeweiligen Bildungssektor beschreibt. Beispielsweise wurde für die Erwachsenenbildung eine Wortgruppe erstellt, um Inhalte spezifisch für diesen Bildungssektor zu erhalten (z. B. Weiterbildung und Lebenslanges Lernen). Die vierte Gruppe „Pädagogisches Personal“ umfasst die wesentlichen Begriffe zu den Berufs- und Personalbezeichnungen der Bildungssektoren.

Die Recherche für das Dossier berücksichtigte den Zeitraum Januar 2010 bis Mai 2019 und wurde auf Deutsch und Englisch durchgeführt. In den Bildungssektoren bestand die Möglichkeit, den Zeitraum weiter einzugrenzen, wenn dies aufgrund inhaltlicher Überlegungen und bildungssektorenspezifischen Begebenheiten angezeigt war. Die Suchwörter wurden sowohl als Schlagwort als auch in Stichwortsuchen kombiniert und passend trunkiert. Für die Suche auf Englisch ließen sich die Begriffe entsprechend übertragen oder treffende Schlagwörter aus den Thesauri der Fachdatenbanken finden, wobei bei der Übersetzung gegebenenfalls eine unterschiedliche Verwendung von Termen in Bezug auf die Bildungssysteme verschiedener Länder berücksichtigt werden muss. Die verwendeten Suchbegriffe sind tabellarisch in der online zugänglichen Dokumentensammlung zum Band aufgeführt.

Die zu Beginn durchgeführten Testsuchen in den Datenbanken halfen einschätzen zu können, wie sinnvoll der Einsatz verschiedener Suchbegriffe und deren Kombinationsmöglichkeiten jeweils ist. Dabei wurden verschiedene Suchterme ausgeschlossen und neue aufgenommen. Beispielsweise eignet sich das Suchwort "Haltung" des pädagogischen Personals nicht für die Datenbankrecherche, da es häufig auch im Kontext von ergonomischen Themen verstanden wird. Deshalb wurde „Haltung" in den meisten Fällen durch den Begriff „Einstellung“ und im Kontext des Thesaurus der FIS Bildung Literaturdatenbank durch den Term "Einstellung" $<$ Psy $>$ ersetzt. Des Weiteren sind nicht alle Suchbegriffe zu digitalen Medien auf jeden Bildungssektor übertragbar. So spielen Begriffe wie "Webinare“ sowie „Blended Learning Kurse“ in der frühkindlichen Bildung im Vergleich zur Erwachsenenbildung in diesem Suchzeitraum keine Rolle. Ebenso wie die unterschiedlichen Sektoren verschiedene Begrifflichkeiten benutzen, muss auch die einheitliche Anwendung und Übertragung der Suchwörter auf die verschiedenen Datenbanken Berücksichtigung finden. So waren die englischen Schlagwörter aus dem Thesaurus von FIS Bildung nicht dieselben wie die Schlagwörter aus dem Thesaurus der Datenbank ERIC und mussten dem damit verknüpften Inhalt entsprechend angepasst werden. Solche Einzelschritte können einen erheblichen zeitlichen Mehraufwand bedeuten.

Für die Recherche in den Datenbanken wurden zu einem Themenschwerpunkt jeweils mehrere Suchanfragen gestellt. Dabei standen die drei Wortgruppen Digitalisierung, Bildungssektor und Pädagogisches Personal immer mit dem jeweiligen 
Themenschwerpunkt in Kombination (z.B. neue Medien UND Kindertagesstätte UND Erzieher UND Fortbildung). Um möglichst viele passende Treffer zu erhalten, beinhalteten die Anfragen sowohl die Suchbegriffe als Schlagwort als auch als Stichwort. Daraus ergaben sich mehrere Suchkombinationen, die jeweils zu den vier Themenschwerpunkten in den fünf Bildungssektoren durchgeführt wurden. Die verschiedenen Suchkombinationen können ebenso in der online verfügbaren Dokumentensammlung nachvollzogen werden. Abbildung 3 zeigt ein Beispiel eines Suchstrings im Fachportal Pädagogik zur beruflichen Bildung zum Themenblock Kompetenz.

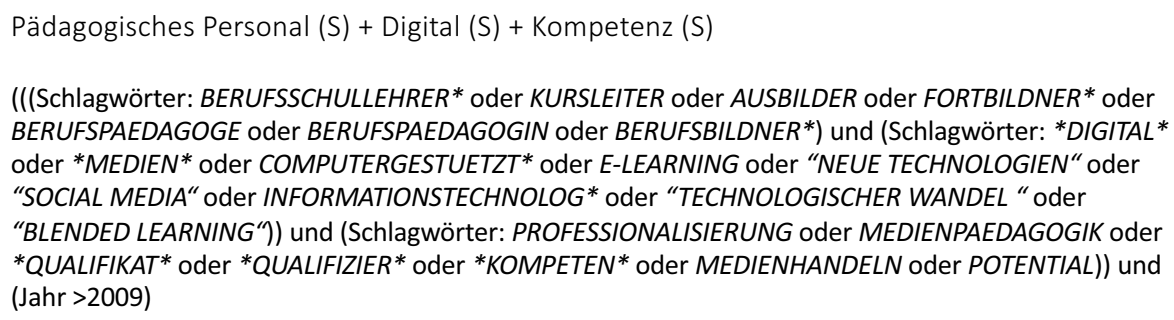

Abbildung 3: Beispiel Suchstring berufliche Bildung in FIS

Nach dem ersten Suchdurchlauf erfolgte in jedem Bildungssektor ein Screening, in welchem die jeweiligen Autorinnen und Autoren die Eignung des Titels für die Fragestellung anhand der Prüfung des Titels und Abstracts bewerteten. Fälle, die sich auf Basis des Abstracts und des Titels nicht eindeutig als passend oder unpassend einordnen ließen, wurden zur weiteren Prüfung im Literaturkorpus belassen. Die Recherchestrategie wurde in Absprache mit den Autorinnen und Autoren der jeweiligen Bildungssektoren weiterentwickelt und die Suchbegriffe sowie deren Kombinationen für einen zweiten Suchdurchlauf angepasst. Zum Beispiel konnte festgestellt werden, dass Suchbegriffe zu Einzelgeräten wie Smartphones oder Tablets ebenso relevant sind wie Überbegriffe bzw. Schlagworte wie Digitalisierung und Handheld Devices. Das liegt vor allem daran, dass die Verschlagwortung der einzelnen Artikel sehr unterschiedlich ausfiel und von der liefernden Institution abhängt. Aus diesem Grund wurde über den Rechercheprozess hinweg kleinteiliger recherchiert und Suchbegriffe wurden ebenso als Schlagwort wie auch in der Freitextsuche verwendet.

Die neuen Titel aus der zweiten Suche wurden den Trefferlisten der ersten Suche ergänzend hinzugefügt. Daraus ergab sich das Literaturkorpus, welches jeweils vom Bildungssektor geprüft und im weiteren Verfahren ausgewertet wurde. Die Treffermengen zu den Recherchen der einzelnen Bildungssektoren können in Tabelle 1 nachvollzogen werden. 
Tabelle 1: Gesamttreffermengen aus der Recherche 2010-2019 nach zwei Suchdurchläufen

\begin{tabular}{c|ccc|c|c|}
\hline Rechercheprozess: & $\begin{array}{c}\text { Frühe } \\
\text { Bildung }\end{array}$ & $\begin{array}{c}\text { Allgemeinbildende } \\
\text { Schule }\end{array}$ & $\begin{array}{c}\text { Berufliche } \\
\text { Bildung }\end{array}$ & $\begin{array}{c}\text { Erwachsenen- } \\
\text { bildung }\end{array}$ & $\begin{array}{c}\text { Lehrer- } \\
\text { bildung }\end{array}$ \\
\hline \hline $\begin{array}{c}\text { Treffermengen aus } \\
\text { Recherchen }\end{array}$ & 1777 & 3380 & 1620 & 2185 & 2636 \\
\hline
\end{tabular}

Der Recherchezeitraum umfasste Februar bis Mai 2019. Da die Datenbanken stets aktualisiert werden, führt die Anwendung der Suchabfrage zu einem späteren Zeitpunkt zu abweichenden Treffermengen. Die Organisation der Treffermengen wie die Prüfung von Dubletten und die Aufbereitung der Daten erfolgte mit dem Literaturverwaltungsprogramm Citavi und dem Tabellenkalkulationsprogramm Excel.

\subsection{Kodierung und Analyse}

Die Kodierung der erfassten Literatur dient in einem ersten Schritt der Sortierung des Feldes nach inhaltlichen und methodischen Schwerpunkten. In einem zweiten Schritt hilft die Kodierung dabei, Forschungsergebnisse zu bündeln, „key concepts“ (Oliver \& Sutcliffe, 2012, S. 139) sowie unterforschte Bereiche zu erfassen und damit die Grundlage für die Beurteilung des untersuchten Forschungsgegenstandes zu schaffen. Für dieses Review wurde ein vergleichsweise offenes Bewertungsraster gewählt, welches ein differenziertes Vorgehen in den Sektoren mit Blick auf Schwerpunktsetzungen und Ausschlussverfahren ermöglichte. Nachdem jedem Sektor eine Literaturliste vorlag, in der eine erste Prüfung anhand der Bewertung von Titel und Abstract bereits stattgefunden hatte, wurde im weiteren Verlauf mit einem dreiteiligen Kodierschema gearbeitet, das sich in Aufbau und Abfrage am methodischen Vorgehen bei Systematic Reviews anlehnt, aber entsprechend der Ausrichtung eines Critical Reviews einen offeneren Umgang mit der Literatur und insbesondere ihrer Auswertung ermöglicht (vgl. Booth, Sutton \& Papaioannou, 2016, S. 24). Kriterien für den Kodierprozess und die sich hieraus ableitende Qualitätsbeurteilung für Systematic Reviews in den Sozialwissenschaften sind zum Beispiel bei Sutcliffe, Oliver und Richardson (2017, S. 123-143), Harden und Gough (2012, S. 153-178) sowie Petticrew und Roberts (2006, S. 125-163) beschrieben. Das EPPI-Centre stellt zudem Guidelines zur umfassenden Beschreibung und Analyse von Studien in der Bildungsforschung als Tool auf seiner Website zur Verfügung (EPPI-Centre, 2003). Die Schwierigkeit, die Methodenvielfalt in der Bildungsforschung adäquat abzubilden, wird in der Literatur reflektiert, indem verschiedene Raster für unterschiedliche Forschungsdesigns wie quantitative, qualitative und Mixed-Methods-Studien und Ausrichtungen der Forschungsarbeiten (z. B. theorieorientiert, praxisnah) diskutiert werden (vgl. Gough, Oliver \& Thomas, 2017; Furlong \& Oancea, 2005). 
Das für dieses Projekt verwendete Kodierschema setzt sich aus den Blöcken Beschreibung der Literatur, Zusammenfassung der Ziele und des Inhalts sowie einem Analyseteil zusammen. Die Verwendung derselben Beurteilungskriterien auf das gesamte Literaturkorpus innerhalb eines Sektors ermöglichte eine systematische Einordnung und Beurteilung der Befunde, auch wenn die Durchführung in einer komprimierten Form stattfand. Beispielsweise war es nicht möglich, die gesamte Literatur von zwei unabhängig voneinander arbeitenden Personen zu prüfen. Ohne Zweifel stärkt eine doppelte Prüfung die Objektivität der Bewertung, ist aber in vielen Fällen aufgrund der vorhandenen Kapazitäten weder umsetzbar noch in jedem Fall nötig (Booth et al., 2016, S. 163; Thomas et al., 2013). Insgesamt konzentrierte sich die Kodierung auf bestimmte im Folgenden vorgestellte zentrale Merkmale, die zur Sortierung und zur Beurteilung der Literatur vom Projektteam sektorenübergreifend als besonders relevant eingestuft wurden.

Der erste Block diente der vollständigen und systematischen Beschreibung der Literatur nach den Kriterien Publikationstyp, Art der Studie und Forschungsdesign sowie einer ersten inhaltlichen Zuordnung nach geographischem Fokus und Kontext der Studie. Daneben wurde für statistische Zwecke auch die Art der Literaturbeschaffung dokumentiert. Im ersten Block bestand erneut die Möglichkeit, Literatur auszuschließen. Gründe hierfür konnten sein, dass die Qualität des Publikationstyps als mangelhaft eingestuft wurde, dass Literatur nicht zugänglich war oder dass durch die Bestimmung des geographischen Bezugs und des Kontexts eine Situation gegeben war, die eine Übertragung in den deutschen Kontext gänzlich ausschloss. Letzteres war zum Beispiel der Fall, wenn die in der Literatur beschriebene Situation weder auf den deutschen Kontext übertragbar war noch einen modellhaften Charakter innehatte. Ein solcher Ausschluss traf vor allem englischsprachige Literatur, die nach dem ersten Screening nach Titel und Abstract noch nicht verworfen werden konnte, sich aber in der Volltextprüfung als eindeutig unpassend kennzeichnen ließ. Da die Studienlage je nach Bildungssektor sehr unterschiedlich ausfiel und auch innerhalb eines Sektors sehr heterogen gelagert sein konnte, wurde im ersten Block keine Beschränkung nur auf bestimmte Studientypen oder Forschungsdesigns vorgenommen, um nicht vor der eigentlichen Qualitätsbeurteilung bestimmte Studiengruppen auszusortieren. Gleichzeitig wurde aber die Möglichkeit eingeräumt, Literatur als kontextbildend einzustufen und somit aus dem Kodierprozess herauszunehmen, wenn sie Hintergründe lieferte, die für die Betrachtung und Rahmung des Themas insgesamt zwar von Interesse waren, sich aber für die weitere inhaltliche Auswertung im Review als ungeeignet erwiesen. Letzteres konnte beispielsweise bei politischen Papieren oder einzelnen Interviews der Fall sein.

Der zweite Block der Inhaltszusammenfassung fragte die Ziele der Studie, Fragestellung und Hypothese sowie Ergebnisse der Literatur ab. In einem weiteren Schritt wurden diese in Bezug zu den thematischen Schwerpunkten des Reviews wie Kompetenz des Personals, Aus- und Fortbildung oder aktuelle Entwicklungen 
gesetzt. Der dritte Block des Kodierschemas diente der Qualitätserfassung und -bewertung unter Berücksichtigung der verschiedenen Studientypen und Ausgangsituationen im Forschungsfeld pro Bildungssektor. Für diesen Analyseteil wurde als Grundlage der Vergleichbarkeit die Kriterien Validität, Reliabilität sowie Generalisierbarkeit und Anwendbarkeit der Studie angelegt (Booth et al., 2016, S. 151-155). Diesen Kategorien wurden wiederum mehrere untergeordnete Fragestellungen zugeordnet, deren Beantwortung jeweils eine Einstufung der Qualität mit den Bewertungen hoch, mittel oder niedrig ermöglichte. $\mathrm{Zu}$ diesen untergeordneten Fragestellungen zählten beispielsweise Fragen zur Nachvollziehbarkeit des Vorgehens, zur Berücksichtigung des Forschungsstandes und zum Bezug zum theoretischen Themenfeld, zum Studiendesign und den jeweiligen Messinstrumenten oder zur Übertragbarkeit allgemein oder speziell zur Anwendung in Politik und Praxis. Die Flexibilität des Bewertungssystems ermöglichte die Berücksichtigung unterschiedlicher Studientypen. Zusätzlich ließ sich festhalten, welche methodisch-spezifischen Indikatoren die Bewertung von quantitativen, qualitativen oder MixedMethods-Studien jeweils beeinflussten. Tabelle 2 stellt den Auswahlprozess nach Sektoren unterteilt dar.

Tabelle 2: Screening-Prozess zur Studienauswahl

\begin{tabular}{|c|c|c|c|c|c|c|}
\hline Screening-Prozess: & & $\begin{array}{l}\text { Frühe } \\
\text { Bildung }\end{array}$ & $\begin{array}{l}\text { Allgemeinbildende } \\
\text { Schule }\end{array}$ & $\begin{array}{l}\text { Berufliche } \\
\text { Bildung }\end{array}$ & $\begin{array}{l}\text { Erwachsenen- } \\
\text { bildung }\end{array}$ & $\begin{array}{l}\text { Lehrer- } \\
\text { bildung }\end{array}$ \\
\hline $\begin{array}{l}\text { Treffermengen aus } \\
\text { Recherchen }\end{array}$ & $\rightarrow$ & 1777 & 3380 & 1620 & 2185 & 2636 \\
\hline \multicolumn{7}{|l|}{$\downarrow$} \\
\hline $\begin{array}{l}\text { Screening basierend auf Titel, } \\
\text { Abstract und Volltext }\end{array}$ & \multirow[t]{2}{*}{$\rightarrow$} & 740 & 2078 & 664 & \multirow[t]{2}{*}{805} & \multirow[t]{2}{*}{1193} \\
\hline$\downarrow$ & & & & & & \\
\hline Ausgeschlossene Titel & $\rightarrow$ & 714 & 1733 & 551 & 746 & 1119 \\
\hline$\downarrow$ & & & & & & \\
\hline Verwendete Titel & \multirow[t]{2}{*}{$\rightarrow$} & 26 & 345 & 113 & 59 & 74 \\
\hline$\downarrow$ & & & & & & \\
\hline Ausgewählte Studien & $\rightarrow$ & 10 & 125 & 14 & 41 & 15 \\
\hline
\end{tabular}

An die Kodierung schließt sich die Darstellung und Analyse der ausgewählten Literatur an. Neben der Generierung von Aussagen zur Be- und Unterforschung bestimmter Themenfelder lassen sich die Ergebnisse miteinander in Verbindung setzen und synthetisieren, um übergeordnete Erkenntnisse zu gewinnen (Thomas, O'Mara-Eves, Kneale \& Shermilt, 2017). Analyseverfahren in narrativ angelegten Reviews können nach Rumrill und Fitzgerald (2001, S. 166) unter anderem zu folgenden Zielsetzungen Auskunft geben: Um theoretische Modelle zu konzipieren oder weiterzuentwickeln, um verschiedene Perspektiven zu kontroversen und kom- 
plexen Fragestellungen zu identifizieren und zu erklären, um Informationen zu BestPractice-Beispielen bereitzustellen und um Perspektiven zu neuen und zunehmend relevanten Themen aufzuzeigen. Ebenso betonen Grant und Booth den konzeptionellen Beitrag von Critical Reviews, denn ein solches „seeks to identify conceptual contribution to embody existing or derive new literature" (Grant \& Booth, 2009, S. 94). Über die Beschreibung der einzelnen Literatur hinaus können diese analytischen Schritte zur Hypothesen- und/oder Modellbildung im Feld beitragen. Je nach Befund kann dies vorrangig bereits bestehende Theorien stärken oder neue Interpretationen anregen (ebd., S. 93). Entsprechend dieser Zielsetzung bietet sich eine nach Themenschwerpunkten gegliederte Darstellungsweise an. ${ }^{15}$

\section{4. Überleitung zu den Reviews der Bildungssektoren}

Die Forschungslage gestaltet sich in jedem der untersuchten Bildungssektoren sehr unterschiedlich gemessen an der Anzahl und Art der vorhandenen Studien wie auch mit Blick auf ihren Entstehungsraum im deutschen oder internationalen Kontext. Insbesondere mit Blick auf Deutschland fällt in mehreren Sektoren eine häufig noch dünne Forschungslage auf. Die Übertragbarkeit auf das deutsche Bildungssystem von Studien, die außerhalb Deutschlands entstanden sind, unterscheidet sich indes je nach Bildungssektor. Beispielsweise fällt dies im Bereich der schulischen Bildung leichter als in der beruflichen Bildung. Sektorenübergreifend zeigt sich, dass individuell geprägte Faktoren den Umgang des pädagogischen Personals mit digitalen Medien mitbestimmen, aber nicht alleine ausschlaggebend sind. Für den Einsatz digitaler Medien spielt die Unterstützung durch die jeweiligen Institutionen ebenfalls eine entscheidende Rolle. In erster Linie gilt dies für einen technischen Support, aber auch für Unterstützung fachspezifischer und methodisch-didaktischer sowie ideeller Art. Zudem kommt der Aus- und Fortbildung eine große Bedeutung zu, wobei idealerweise $\mathrm{zu}$ berücksichtigen ist, welche Formate sich für die Vermittlung digitaler Kompetenzen als besonders vorteilhaft erweisen und auch Möglichkeiten über traditionelle Fortbildungen hinaus mitgedacht werden sollten. Im Sektor Schule hebt Bettina Waffner beispielsweise die größere Bedeutung hervor, die Massive Open Online Courses (MOOCs) und Twitter schon jetzt im nichtdeutschen Bereich für die Fortbildung besitzen. Neben dem Schulsektor verweisen zum Beispiel auch die Reviews zur Lehrerausbildung und zur Erwachsenen- und Weiterbildung auf den erfolgreichen Einsatz von langfristig angelegten Mentoren- und Peer-Learning Programmen.

Für den Bereich der frühen Bildung können Iris Nieding und E. Katharina Klaudy aufzeigen, dass die Aspekte Haltung, Einstellung und Kompetenz eng an die Aus- und Fortbildung von Erzieherinnen und Erziehern geknüpft sind. Die im

15 Vgl. den inhaltlich orientierten Aufbau beim Critical Review von Kulviwat, Guo \& Engchanil, 2004 oder auch im Review von Uerz, Volman \& Kral, 2018. 
Review rezipierten empirischen Studien weisen darauf hin, dass Medienbildung und Medienkompetenzen bei frühpädagogischen Fachkräften noch unzureichend vorhanden sind. Obwohl eine theoretische Medienbildung in manchen Bundesländern in die Curricula der Ausbildung verankert ist, fehlt es an praktischen Modellen und Umsetzungen in der Praxis. Im Sektor Schule kann Bettina Waffner nachzeichnen, dass Lehrkräfte digitale Medien im Berufsalltag bereits verwenden, um Inhalte zu präsentieren und somit traditionelle Unterrichtsformen zu unterstützen oder zu ergänzen und weniger in Form von pädagogisch-didaktischen Konzepten der Unterrichtsgestaltung. Entsprechend verweist Waffner auf die Notwendigkeit einer Kompetenzschulung, die über das technische Wissen hinausgeht und Didaktik und neue Kommunikationsmöglichkeiten berücksichtigt. In den Studien, die Marcel Capparozza und Gabriele Irle für den Sektor Lehrerbildung auswerten, werden die Kompetenzen von Lehrerausbildenden in Bezug auf digitale Medien deutlich stärker thematisiert als die Frage nach ihrer Einstellung zu digitalen Medien. Capparozza und Irle können anhand der erfassten Kompetenzmodelle ebenfalls nachzeichnen, wie wichtig die Kombination von pädagogischen und technischen Kompetenzen für den Einsatz digitaler Medien ist.

Aufgrund der Arbeitsmarktnähe sind die institutionellen Anpassungsanforderungen an die Berufsbildungspraxis, die durch eine digitalisierte Arbeitswelt gestellt werden, besonders hoch. Ausbildungspersonal und Lehrkräfte sind hierbei als Schlüsselakteure zu verstehen. Die Studien, die für diesen Bereich von Katharina Hähn und Monique Ratermann-Busse ausgewertet wurden, verweisen auf Unterschiede in der technischen Ausstattung der Betriebe, der Berufsschulen und Ausbildungsstätten sowie bei personellen Ressourcen. Die Autorinnen stellen heraus, dass es an flächendeckenden Qualifizierungsangeboten und an einer flächendeckenden strukturellen Verankerung von Fort- und Weiterbildungsformaten zum Einsatz digitaler Medien in den Bildungsinstitutionen und Ausbildungsbetrieben fehlt. Auch die mangelnde bzw. nicht geprüfte Qualität, die begrenzte Verbreitung von speziellen Lernprogrammen sowie die fehlende Entwicklung fach-didaktischer Szenarien hindern den Einsatz digitaler Medien durch das Lehr- und Ausbildungspersonal. Schließlich zeigt auch der Sektor Erwachsenenbildung und Weiterbildung eine große Heterogenität, die sich in der Vielfältigkeit der Anbieter und Programme widerspiegelt. Trotz einer allgemein hohen Fortbildungsaffinität in diesem Sektor verweisen Jan Koschorrek und Angelika Gundermann auf die noch nicht ausreichenden digitalen Kompetenzen vieler Lehrender. Die Berücksichtigung vorhandener Kompetenzmodelle wie Greta oder DigCompEdu kann die Umsetzung von Qualifizierungsmaßnahmen in der Erwachsenen- und Weiterbildung unterstützen. Medienpädagogische Kompetenzen der Lehrenden lassen sich auch in diesem Sektor als Gelingensbedingungen für digitales Lernen und Lehren identifizieren, wobei sich die Rolle der Lehrenden von Wissensvermittelnden zu Lernbegleitenden wandelt.

Die Reviews der einzelnen Bildungssektoren bieten einen Einblick in die jeweils aktuellen Forschungsdebatten, verweisen auf Forschungsbedarfe und mögliche 
Implikationen für die Praxis. Bei der Frage nach der Bedeutung von Digitalisierung für das pädagogische Personal in unterschiedlichen Bildungsbereichen ist zu erwarten, dass sich die Forschung im Themenfeld dynamisch weiterentwickeln wird. Die hier identifizierten Forschungsstränge und benannten Forschungslücken können dazu beitragen, das Feld weiter auszugestalten.

\section{Literatur}

Beelmann, A. (2014). Möglichkeiten und Grenzen systematischer Evidenzkumulation durch Forschungssynthesen in der Bildungsforschung. Zeitschrift für Erziehungswissenschaft, 17(S4), 55-78. https://doi.org/10.1007/s11618-014-0509-2

Blossfeld, H.-P., Bos, W., Daniel, H.-D., Hannover, B., Köller, O., Lenzen, D. et al. (2018). Digitale Souveränität und Bildung. Gutachten (1. Auflage). Münster: Waxmann.

$\mathrm{BMBF}=$ Bundesministerium für Bildung und Forschung (Hrsg.). (2008). Wissen für Handeln - Forschungsstrategien für eine evidenzbasierte Bildungspolitik. Fachtagung im Rahmen der deutschen EU-Ratspräsidentschaft. Bonn, Berlin.

$\mathrm{BMBF}=$ Bundesministerium für Bildung und Forschung (Hrsg.). (2016). Bildungsoffensive für die digitale Wissensgesellschaft. Strategie des Bundesministeriums für Bildung und Forschung. Berlin.

Booth, A. (2010). How much searching is enough? Comprehensive versus optimal retrieval for technology assessments. International journal of technology assessment in health care, 26(4), 431-435. https://doi.org/10.1017/S0266462310000966

Booth, A., Sutton, A. \& Papaioannou, D. (2016). Systematic Approaches to a Successful Literature Review (2. Auflage). Los Angeles: SAGE.

Burow, O.-A. (2019). Schule digital - Wie geht das? Wie die digitale Revolution uns und die Schule verändert. Weinheim, Basel: Beltz.

Davies, P. (2004). Systematic Reviews and the Campbell Collaboration. In G. Thomas \& R. Pring (Hrsg.), Evidence-based practice in education (S. 21-33). Maidenhead: Open University Press.

Deutscher Bundestag / Ausschuss für Bildung, Wissenschaft, Forschung, Technologie und Technikfolgenabschätzung (Hrsg.). (2016). Technikfolgenabschätzung (TA): Digitale Medien in der Bildung. Bericht des Ausschusses für Bildung, Forschung und Technikfolgenabschätzung (18. Ausschuss) gemäß $\$ 56 a$ der Geschäftsordnung. (Drucksache / Deutscher Bundestag. 18/9606 v. 08.09.2016). Berlin.

Dixon-Woods, M., Bonas, S., Booth, A., Jones, D. R., Miller, T., Sutton, A. J. et al. (2006). How can systematic reviews incorporate qualitative research? A critical perspective. Qualitative Research, 6(1), 27-44. https://doi.org/10.1177/1468794106058867

EPPI-Centre. (2003). Review Guidelines for Extracting Data and Quality Assessing Primary Studies in Educational Research. Version 0.9.7, EPPI-Centre. https://eppi.ioe.ac.uk/ CMS/Portals/0/PDF\%20reviews\%20and\%20summaries/Guidelines\%20for\%20extracting\%20data\%20and\%20quality\%20assessing\%20primary\%20studies\%20in\%20educational\%20research\%20Version\%200.97_2.doc

Europäische Kommission (Hrsg.). (2018). Mitteilung der Kommission an das Europäische Parlament, den Rat, den Europäischen Wirtschafts- und Sozialausschuss und den Ausschuss der Nationen zum Aktionsplan für digitale Bildung (Rat für Bildung, Jugend, Kultur und Sport). Brüssel. https://eur-lex.europa.eu/legal-content/DE/TXT/PDF/?uri= CELEX:52018DC0022\&from=EN 
Europäische Kommission (Hrsg.). (2020). Communication from the European Mission to the European Parliament, the Council, the European Economic and Social Commitee of the Regions: A European Strategy for Data. Brüssel. https://ec.europa.eu/info/sites/info/ files/communication-european-strategy-data-19feb2020_en.pdf

Fink, A. (2005). Conducting research literature reviews. From the Internet to paper (2. Auflage). Thousand Oaks, California: Sage Publications.

Furlong, J. \& Oancea, A. (2005). Assessing quality in applied and practice-based educational research: A framework for discussion. Australian Educational Researcher, 6, 89-104.

Gough, D. (2009). Qualitative, Quantitative and Mixed Methods Systematic Reviews to Support Professional Decision Making in Education. In W. Böttcher, J. N. Dicke \& H. Ziegler (Hrsg.), Evidenzbasierte Bildung. Wirkungsevaluation in Bildungspolitik und pädagogischer Praxis (S. 23-33). Münster: Waxmann.

Gough, D., Oliver, S. \& Thomas, J. (Hrsg.). (2012). An Introduction to Systematic Reviews. Los Angeles: SAGE.

Gough, D., Oliver, S. \& Thomas, J. (Hrsg.). (2017). An Introduction to Systematic Reviews (2. Auflage). Los Angeles: SAGE.

Gough, D. \& Thomas, J. (2016). Systematic reviews of research in education. Aims, myths and multiple methods. Review of Education, 4(1), 84-102. https://doi.org/10.1002/ rev3.3068

Grant, M. J. \& Booth, A. (2009). A typology of reviews. An analysis of 14 review types and associated methodologies. Health information and libraries journal, 26(2), 91-108. https://doi.org/10.1111/j.1471-1842.2009.00848.x

Harden, A. \& Gough, D. (2012). Quality and relevance appraisal. In D. Gough, S. Oliver \& J. Thomas (Hrsg.), An Introduction to Systematic Reviews (S. 153-178). Los Angeles: SAGE.

Kerres, M. (2003). Zu Wirkungen und Risiken neuer Medien in der Bildung. Warum Medien keine Arznei für die Bildung sind. In A. Schlüter (Hrsg.), Aktuelles und Querliegendes zur Didaktik und Curriculumentwicklung. Festschrift für Werner Habel (S. 261-278). Bielefeld: Janus Verlag.

Kirscher, P. A., Sweller, J. \& Clark, R. E. (2006). Why minimal guidance during instruction does not work: An analysis of the failure of constructivist, discovery, problembased, experiential, and inquiry based teaching. Educational Psychologist, 41(2), 75-86. https://doi.org/10.1207/s15326985ep4102_1

KMK = Ständige Konferenz der Kultusminister der Länder in der Bundesrepublik Deutschland (Hrsg.). (2017). Strategie der Kultusministerkonferenz „Bildung in der digitalen Welt“. Berlin.

Kulik, J. A., Kulik, C.-L. C. \& Cohen, P. A. (1980). Effectiveness of computer-based college teaching. A meta-analysis of findings. Review of Educational Research, 50(4), 525-544. https://doi.org/10.3102/00346543050004525

Kulviwat, S., Guo, C. \& Engchanil, N. (2004). Determinants of online information search. A critical review and assessment. Internet Research, 14(3), 245-253. https://doi.org/ $10.1108 / 10662240410542670$

Newman, M. \& Gough, D. (2020). Systematic Reviews in Educational Research: Methodology, Perspectives and Application. In O. Zawacki-Richter, M. Kerres, S. Bedenlier, M. Bond \& K. Buntins (Hrsg.), Systematic Reviews in Educational Research (S. 3-22). Wiesbaden: Springer Fachmedien Wiesbaden.

Oakley, A., Gough, D., Oliver, S. \& Thomas, J. (2005). The politics of evidence and methodology. Lessons from the EPPI-Centre. Evidence \& Policy: A Journal of Research, Debate and Practice, 1(1), 5-32. https://doi.org/10.1332/1744264052703168 
Oliver, S. \& Sutcliffe, K. (2012). Describing and analysing studies. In D. Gough, S. Oliver \& J. Thomas (Hrsg.), An Introduction to Systematic Reviews (S. 135-152). Los Angeles: SAGE.

Petticrew, M. (2015). Time to rethink the systematic review catechism? Moving from 'what works' to 'what happens.' Systematic Reviews, 4, 36. https://doi.org/10.1186/s13643-0150027-1

Petticrew, M. \& Roberts, H. (2006). Systematic reviews in the social sciences. A practical guide. Oxford: Wiley. https://doi.org/10.1002/9780470754887

Polanin, J. R., Maynard, B. R. \& Dell, N. A. (2017). Overviews in Education Research. Review of Educational Research, 87(1), 172-203. https://doi.org/10.3102/0034654316631117

Redecker, C. \& Punie, Y. (2017). European Framework for the Digital Competences of Educators. DigCompEdu. Brüssel: Publication Office of the European Union. (Dt. Übersetzung vom Goethe Institut 2019).

Rumrill, P. D. \& Fitzgerald, S. M. (2001). Using narrative literature reviews to build a scientific knowledge base. Work (Reading, Mass.), 16(2), 165-170.

Sutcliffe, K., Oliver, S. \& Richardson, M. (2017). Describing and Analysing Studies. In D. Gough, S. Oliver \& J. Thomas (Hrsg.), An Introduction to Systematic Reviews (2. Auflage, S. 123-143). Los Angeles: SAGE.

Tamim, R. M., Bernard, R. M., Borokhovski, E., Abrami, P. C. \& Schmid, R. F. (2011). What forty years of research says about the impact of technology on learning. A second-order meta-analysis and validation study. Review of Educational Research, 81(1), 4-28. https://doi.org/10.3102/0034654310393361

Thomas, J., Newman, M. \& Oliver, S. (2013). Rapid evidence assessments of research to inform social policy. Taking stock and moving forward. Evidence \& Policy: A Journal of Research, Debate and Practice, 9(1), 5-27. https://doi.org/10.1332/174426413X662572

Thomas, J., O’Mara-Eves, A., Kneale, D. \& Shemilt, I. (2017). Synthesis Methods for Combining and Configuring Textual or Mixed Methods Data. In D. Gough, S. Oliver \& J. Thomas (Hrsg.), An Introduction to Systematic Reviews (2. Auflage, S. 181-209). Los Angeles: SAGE.

Uerz, D., Volman, M. \& Kral, M. (2018). Teacher educators' competences in fostering student teachers' proficiency in teaching and learning with technology. An overview of relevant research literature. Teaching and Teacher Education, 70, 12-23. https://doi. org/10.1016/j.tate.2017.11.005

Zawacki-Richter, O., Kerres, M., Bedenlier, S., Bond, M. \& Buntins, K. (Hrsg.). (2020). Systematic Reviews in Educational Research. Wiesbaden: Springer Fachmedien Wiesbaden. https://doi.org/10.1007/978-3-658-27602-7 

Iris Nieding \& E. Katharina Klaudy

\title{
Digitalisierung in der frühen Bildung \\ Der Umgang mit digitalen Medien im Spannungsfeld zwischen Schutzraum und Schlüsselkompetenz
}

\begin{abstract}
Das erste Dossier zum Thema „Bildung im Digitalen Wandel“ beschäftigt sich mit der Bedeutung der Digitalisierung für das pädagogische Personal, in diesem Kapitel mit Schwerpunkt im Bereich der frühkindlichen Bildung. Dabei spielen besonders die Themen der Haltung und Kompetenz der Fachkräfte in Kindertageseinrichtungen eine wichtige Rolle und stehen gleichzeitig in einem engen Verhältnis zu der Aus- und Weiterbildung der Erzieher und Erzieherinnen. Wie sich diese Zusammenhänge genauer ausgestalten, wird mittels einer narrativen Synthese von zehn empirischen Studien der vergangenen Jahre differenziert dargelegt. Die Studien wurden anhand festgelegter Kriterien kodiert, bewertet und abschließend nach übergeordneten inhaltlichen Aspekten (Haltung, Kompetenz, Aus- und Fortbildung) aufbereitet.

Schlüsselwörter: frühe Bildung, Digitalisierung, Medienerziehung, Medienbildung, Kindertageseinrichtung, Fortbildung, Ausbildung, Kompetenz, Haltung, pädagogische Fachkraft
\end{abstract}

\section{Digitisation in early education. Dealing with digital media in the conflict between protected space and key competence}

The first review of "Education in an era of digital change" deals with the importance of digitisation for educational staff. This chapter focuses on early childhood education. The issues of attitude and competence of professionals in day-care facilities play a particularly important role in this context, and they are also closely related to the initial and continuing training of educators. A narrative synthesis of ten empirical studies from recent years provides a differentiated account of how these interrelationships developed in detail. Criteria were defined to code and evaluate the studies, which were finally processed according to superordinate content aspects (attitude, competence, training and further education).

Keywords: early education, digitisation, media education, digital literacy, day care centre, training, vocational education, competence, attitude, kindergarten

\section{Einführung: Aktuelle Entwicklungen}

Das seit den 2000er Jahren in Deutschland genutzte Leitbild der „Bildung von Anfang an" verläuft im Sinne der Selbstbildung begleitend in verschiedenen Lebensphasen. Im Kontext institutioneller Bildung stellen Kindertageseinrichtungen die 
erste Station der Bildungskette junger Menschen dar. Im Gegensatz zu anderen Bildungsbereichen wird der Einsatz digitaler Medien in Kindertageseinrichtungen kontrovers diskutiert und ist im Vergleich zu nachfolgenden Bildungsinstitutionen (wie bspw. der Schule) nur wenig beforscht, obwohl die Lebenswelt sehr junger Kinder längst digital geprägt ist. Nolte (2014) und Theunert (2007) sprechen in diesem Zusammenhang von einer "Quasi-Omnipräsenz", da sich die Mediatisierung nicht nur in Form von technischen Geräten mit der Welt der Kinder verwebt, sondern sich auch über die konsumierten Inhalte und daraus resultierenden Interessen manifestiert (Nolte, 2014, S. 214). Eine ähnliche Entwicklung belegt die „miniKIM Studie, Kleinkinder und Medien, Basisuntersuchung zum Medienumgang 2- bis 5-Jähriger in Deutschland", die das Mediennutzungsverhalten von Kindern im Alter von zwei bis fünf Jahren untersucht (Feierabend et al., 2015). Kinder wachsen unabhängig von der Ausstattung und ihres sozialen Hintergrunds nicht medienfern auf und haben in ihrer Lebenswelt häufigen Kontakt mit digitalen Medien und Geräten (Feierabend, Plankenhorn \& Rathgeb, 2015, S. 32).

Der Einsatz digitaler Medien vollzieht sich in unterschiedlichen Handlungsfeldern der Frühpädagogik, z. B. in der musikalischen Erziehung, beim forschenden Lernen und in der Medienerziehung und steht vor der Herausforderung, Kompetenzen zu fördern und Kinder zu befähigen, sich in einer zunehmend komplexen Welt zurechtzufinden. Ein kompetenter Medienumgang sollte demnach bereits in der frühen Kindheit geschaffen werden, wobei die Begleitung durch Erzieher und Erzieherinnen und Eltern eine wesentliche Rolle spielt. Der Auftrag von pädagogischen Fachkräften besteht darin, Kinder an einen verantwortungsbewussten und reflexiven Umgang mit diesen Medien heranzuführen und ihre Fähigkeiten zu stärken, sich vor möglichen Gefahren zu schützen und die Vorteile für sich selbst zu nutzen. Diese Haltung vertreten auch die Initiative „Keine Bildung ohne Medien“ (KBoM, Büsch \& Demmler, 2017) und die Gesellschaft für Medienpädagogik und Kommunikationskultur (GMKFachgruppe Kita, Eder, Brüggemann \& Kratzsch, 2017) in ihren Positionspapieren zur Integration von Medienpädagogik in die frühkindliche Bildung.

Auch Stimmen aus Wissenschaft und Forschung äußern sich in Fachartikeln und Beiträgen zu der Notwendigkeit, Medienbildung nicht zu ignorieren, sondern kompetent zu begleiten. Bostelmann betont die nötige kritische Reflexivität der Fachkräfte und die Nutzung digitaler Medien als Unterstützungsinstrument für die Umsetzung des Bildungsauftrages im pädagogischen Alltag (Bostelmann, 2018). In der Praxis gibt es bereits Stiftungen und Vereine, die sich den Herausforderungen durch die Digitalisierung widmen: Die Stiftung „Haus der kleinen Forscher"1 bietet z. B. Fortbildungs- und Zertifizierungsangebote für Fachkräfte in Deutschland im MINT-Bereich an (Graube et al., 2015, S. 43).

Neben der Medienerziehung im pädagogischen Alltag bildet der steigende Informations- und Beratungsbedarf von Familien zur Nutzung digitaler Medien ein weiteres Arbeitsfeld der Fachkräfte. Für die Einrichtungen selbst stellen sich zu-

1 https://www.haus-der-kleinen-forscher.de/ 
sätzlich Fragen der Qualitätsentwicklung und des rechtlichen Rahmens von Kitas ${ }^{2}$ und Erzieher und Erzieherinnen im Umgang mit persönlichen Daten und dem Datenschutz. Reichert-Garschhammer prognostiziert einen umfassenden digitalen Transformationsprozess, der sich über alle Handlungsfelder einer Kita erstreckt und sich bereits seit mehreren Jahren mit immer schneller werdender Geschwindigkeit vollzieht (Reichert-Garschhammer, 2019, S. 26).

Unabhängig davon, wie intensiv digitale Medien von Kindern genutzt werden, stellt sich auf Seiten der Fachkräfte heraus, dass diese als Störfaktoren oder negative Einflüsse wahrgenommen werden. Die oft thematisierte "bewahrpädagogische“ Haltung der Fachkräfte ist auf den negativ geführten gesellschaftlichen Diskurs zurückzuführen, der sich vor allem durch Negativschlagzeilen und Risikofokussierung auszeichnet (Neuß \& Wiechmann, 2017, S. 223).

Auch der Aus- und Weiterbildung kommt hier eine wichtige Rolle zu: In ihr wird ein zentrales Element für den Erwerb von informatischer, technischer oder medienpädagogischer Bildung der Fachkräfte gesehen, die sowohl Auswirkungen auf deren Kompetenzen als auch auf deren Einstellungsmuster haben (Bergner et al., 2018, S. 210). Die Faktoren Haltung gegenüber digitalen Medien und Kompetenzen sowie Fortbildung zu neuen Medien sind in diesem Feld eng miteinander verwoben und beeinflussen sich gegenseitig. Das Dossier geht den Fragen nach, wie sich Haltung und Kompetenzen der Fachkräfte darstellen und welche Implikationen sich daraus für die Aus- und Fortbildung der Fachkräfte ergeben.

\section{Methodik}

Der erste Rechercheprozess durch das Leibniz-Institut für Bildungsforschung und Bildungsinformation (siehe Kapitel 1 in diesem Band) führte für den Sektor der frühen Bildung zu 1.777 Literaturtiteln aus den Jahren 2010 bis 2019. Das Screening machte deutlich, dass besonders viele englischsprachige Treffer dem schulischen Bereich zuzuordnen waren und dadurch ausgeschlossen werden konnten. Dies ist der eingeschränkten Übertragbarkeit auf die unterschiedlichen Systeme und Strukturen der frühen Bildung, Betreuung und Erziehung in den OECDLändern geschuldet. Während deutschsprachige Literatureinträge mit den Begriffen Erzieher und Erzieherinnen und pädagogische Fachkräfte verschlagwortet sind, werden sie in den englischen Datenbanken unter dem Begriff teacher subsummiert. Weitere Kollisionen entstanden mit den Bezeichnungen und der Einordnung der Bildungsinstitutionen in die Bildungssysteme der Länder. In den „International Standard Classification of Education" besteht der Bildungsbereich 0 (early childhood education) neben der formalen frühkindlichen Bildung für Kinder ab drei Jahren

2 Nachfolgend werden Kindertageseinrichtungen, Kindertagesstätten, Kindergärten und Kinderkrippen als „Kita" bezeichnet. 
bis zum regulären Schuleintritt auch aus dem frühkindlichen Bildungsbereich für Kinder bis zu drei Jahren (BIBB, 2012, S. 18).

\subsection{Screening}

Die hohe Zahl der gefundenen Titel der letzten zehn Jahre machte für den Arbeitsprozess eine weitere Einschränkung nötig. So wurde der zu analysierende Zeitraum auf die Jahre 2016-2019 beschränkt. Ein Rückgriff auf frühere Veröffentlichungen war weiterhin möglich, wenn es sich dabei um bspw. häufig zitierte Literatur oder Grundlagenwerke in dem Sektor handelte. Für den Zeitraum 2016-2019 wurden 740 Literatureinträge inklusive Abstracts gesichtet. Davon erwiesen sich insgesamt 688 als nicht relevant für das Dossier (siehe Abbildung 1) und 185 Einträge wurden aufgrund fehlender Passung zum Bildungssektor aussortiert. Ein weit größerer Teil, mit 395 Literatureinträgen, wurde aussortiert, weil sie zwar dem richtigen Bildungssektor zugehörig waren, sich aber thematisch mit anderen Schwerpunkten oder Zielgruppen befassten, wie bspw. mit den Eltern oder der Mediennutzung der Kinder. Nicht berücksichtigt wurde auch Literatur, die sich mit der Erprobung bestimmter Lernsoftwares oder digitaler Instrumente in den Einrichtungen beschäftigte. Bei 70 Literatureinträgen führte, trotz thematischer und sektorspezifischer Passung, der geografische Fokus zum Ausschluss, da sich das System der frühen Bildung in Deutschland nur schwer mit dem unterschiedlich aufgebauten Elementarbereich im internationalen Kontext vergleichen lässt. Unter dem Kriterium Dokumententyp wurden unter anderem Abschlussarbeiten und Internetseiten aussortiert. Von den 740 Treffern ab 2016 wurden somit 26 Veröffentlichungen für das Dossier berücksichtigt. Um die Grundlage für das Dossier mit weiteren Publikationen anzureichern, wurden aus dem Zeitraum 2010-2016 sieben weitere Veröffentlichungen hinzugenommen und vier Publikationen nachträglich ergänzt, die von den Autorinnen als relevant für das Dossier erachtet wurden, aufgrund der Verschlagwortung aber in dem Screening-Prozess nicht auffindbar waren und zum Teil sowohl vor als auch nach dem Suchzeitraum veröffentlicht wurden. In die Bearbeitung flossen somit 37 Texte ein. 


\begin{tabular}{|c|c|c|c|c|c|}
\hline \multicolumn{6}{|c|}{ Recherche 2010-2019 } \\
\hline \multicolumn{6}{|c|}{ 1.777 Treffer } \\
\hline \multicolumn{6}{|c|}{ Fokus 2016-2019 } \\
\hline \multicolumn{6}{|c|}{740 Titel waren vorhanden und wurden anhand der Abstracts geprüft } \\
\hline \multicolumn{6}{|c|}{ Ausschlusskriterien } \\
\hline $\begin{array}{l}\text { passt nicht zum } \\
\text { Bildungssektor } \\
\text { (185 Titel) }\end{array}$ & $\begin{array}{l}\text { thematisch un- } \\
\text { passend } \\
\text { (395 Titel) }\end{array}$ & $\begin{array}{l}\text { geografischer } \\
\text { Fokus nicht } \\
\text { übertragbar } \\
\text { (70 Titel) }\end{array}$ & \multicolumn{2}{|c|}{$\begin{array}{l}\text { Sonstiges } \\
\text { (58 Titel) }\end{array}$} & $\begin{array}{l}\text { Qualität zu gering } \\
\text { (6 Titel) }\end{array}$ \\
\hline \multicolumn{6}{|c|}{ Auswahl } \\
\hline \multicolumn{2}{|c|}{ Aus dem Rechercheprozess } & \multicolumn{4}{|c|}{ Ergänzung } \\
\hline \multicolumn{2}{|c|}{26 Titel } & \multicolumn{2}{|c|}{$\begin{array}{l}\text { Aus dem Zeitraum 2010- } \\
2015 \text { wurden } 7 \text { Titel basie- } \\
\text { rend auf ihrer Bedeutung } \\
\text { in späteren Werken hinzu- } \\
\text { genommen }\end{array}$} & \multicolumn{2}{|c|}{$\begin{array}{c}\text { 4 Titel waren Ergebnis des } \\
\text { Rechercheprozesses und } \\
\text { wurden von den Autorinnen } \\
\text { hinzugefügt }\end{array}$} \\
\hline \multicolumn{6}{|c|}{ Finale Auswahl für die Kodierung } \\
\hline & & 37 Titel & & & \\
\hline
\end{tabular}

Abbildung 1: Screening-Prozess

Quelle: eigene Darstellung

\subsection{Kodierung}

In Anlehnung an ein Critical Review wurde die zugrunde gelegte Literatur anhand des in Kapitel 1 festgelegten Kodierschemas nach verschiedenen Kriterien bewertet (siehe Kapitel 1 in diesem Band von Wilmers et al.). Im ersten Schritt der Kodierung wurden alle relevanten Publikationen für eine allgemeine Beschreibung einbezogen, während im zweiten Schritt ausschließlich empirische Studien berücksichtigt und anhand inhaltlicher Kriterien bewertet wurden. Im ersten Arbeitsschritt der Kodierung wurde die Literatur genauer spezifiziert. Die Kriterien dafür bildeten der Publikationstyp, die Art der Studie, der geografische Fokus und Themenschwerpunkte. Die grobe Gliederung der Themenschwerpunkte war für alle am Prozess beteiligten Bildungssektoren identisch und untergliederte sich in Haltung und Rolle, Kompetenz sowie Aus- und Fortbildung. 
Thematisch lassen sich acht Veröffentlichungen dem Bereich „Persönliche Haltung/Rolle" zuordnen, 15 Publikationen haben unter anderem die Kompetenzen des pädagogischen Personals zum Thema und weitere 14 Titel beschäftigen sich mit der Aus- und Fortbildung der frühpädagogischen Fachkräfte. Es gibt selten Literatur, die ausschließlich einen Bereich thematisiert, ohne die beiden anderen Kernthemen einzubeziehen.

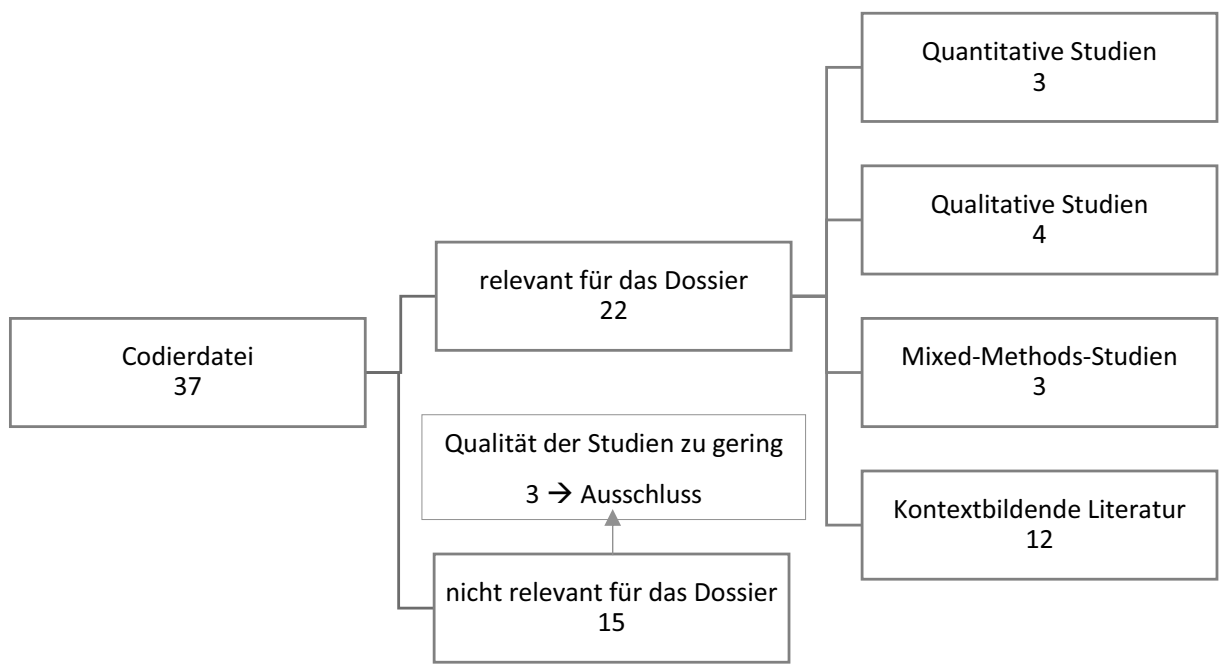

Abbildung 2: Kodierung

Quelle: eigene Darstellung

Der zweite Schritt im Ablauf des Kodierens beinhaltete eine nähere Beschäftigung mit den inhaltlichen Aspekten der empirischen Studien, deren konzeptioneller Aufbau, Fragestellungen, methodische Herangehensweise sowie der Qualitätserfassung wissenschaftlicher Kriterien, wie bspw. der Validität des Vorgehens oder die Anwendbarkeit der Studienergebnisse auf das Forschungsfeld des Bildungssektors frühe Bildung. Die Bewertungskriterien, die nach der Kodierung für jede Studie vorlagen, bilden die Grundlage für einen zentralen Überblick über die Studienlandschaft zum Thema Digitalisierung in Kitas in Hinblick auf die Herausforderungen für das pädagogische Personal. Von den 37 ausgewählten Literaturtiteln erwiesen sich bei einer erneuten Prüfung 15 als nicht relevant für das Dossier. Dies lag größtenteils an einer zunächst nicht sichtbaren, aber nach Kodierung des gesamten Volltextest festgestellten fehlenden inhaltlichen Passung der Titel. Drei Studien wurden aufgrund mangelnder Qualität ausgeschlossen. 22 Veröffentlichungen wurden als relevant für das Dossier eingestuft. Davon bildeten zwölf Literatureinträge einen inhaltlichen Rahmen für die thematische Einordnung (kontextbildende Literatur). Als Ergebnis des Bewertungsprozesses wiesen zehn Studien eine ausreichende Qualität auf, um in das Dossier aufgenommen werden zu können. 


\subsection{Beschreibung der Studien}

Die zugrunde gelegten Studien (siehe Tabelle 1) enthalten größtenteils mindestens zwei der drei Schwerpunktthemen Haltung, Kompetenz oder Aus- und Weiterbildung. Dass fünf Studien alle drei Kernbereiche in ihrer Fragestellung berücksichtigen, unterstreicht, wie eng diese Aspekte inhaltlich miteinander verwoben sind. Bei den quantitativen Studien werden überwiegend Fragebogenerhebungen eingesetzt, während bei qualitativen Studien Interviews und Gruppendiskussionen genutzt werden. Die Mixed-Methods Studien kennzeichnen sich dadurch, dass zusätzlich zu einer Fragebogenerhebung entweder Gruppendiskussionen geführt oder Dokumente inhaltsanalytisch untersucht werden. Das Vorgehen der Studien ist größtenteils explorativer Natur und versucht für den bundeslandspezifischen Fokus eine Bestandsaufnahme zu bestimmten Punkten zu generieren. Die forschungsleitenden Fragestellungen zielen darauf ab, einen Überblick über Ausstattungen, Kompetenzwahrnehmungen, Qualifizierungen der Fachkräfte (durch die Abfrage von Ausbildungsinhalten oder Inanspruchnahme von Fortbildungen) oder einrichtungs-, bzw. trägerspezifische Rahmenbedingungen zu liefern. Dabei wird der Fokus häufig auf Nordrhein-Westfalen gelegt und vereinzelt auf die Bundesländer Berlin, Bayern, Niedersachsen, Baden-Württemberg und Sachsen-Anhalt. Lediglich eine Studie hat eine bundesweite Fragebogenerhebung im Rahmen eines größeren Projektes (Weiterbildungsinitiative Frühpädagogische Fachkräfte, WiFF-Befragung) durchgeführt (Gessler \& Gruber, 2018) und drei Studien untersuchten durch Dokumentenanalysen Ausbildungs- und Bildungspläne in ganz Deutschland (FriedrichsLiesenkötter, 2019; Meister, Friedrichs, Keller, Pielsticker \& Temps 2012; Six \& Gimmler, 2007).

Die Fallzahlen, die den Studien aus den vergangenen Jahren zugrunde liegen, weisen eine hohe Spannweite auf. Die niedrigste Fallzahl fand sich in einer Dissertation (Friedrichs-Liesenkötter, 2016). Dies mindert jedoch nicht die methodische Qualität der Studie, da besonders auch qualitativ angelegte Einzelfallstudien im wenig erforschten Feld der frühen Bildung wertvolle Hinweise für vorhandene Bedingungsgefüge liefern können. Den Fallzahlen entsprechend setzen die Autoren und Autorinnen überwiegend deskriptive Verfahren ein, um die Bestandsaufnahmen abzubilden. Die qualitativen Forschungsarbeiten nutzen dafür inhaltsanalytische Auswertungsmethoden. Lediglich zwei Studien, eine mit vergleichsweiser hoher Fallzahl, setzen neben deskriptiven Verfahren multivariate Berechnungen ein, um Zusammenhänge und Einflussgrößen von Mediennutzung oder Medieneinsatz, medienpädagogischer Kompetenz und Qualifizierung der Fachkräfte zu berechnen (Gessler \& Gruber, 2018; Nolte, 2014). Ein weiteres wichtiges Element der Forschung zu den Ausgangslagen und -bedingungen in Kitas stellen Dokumentenanalysen dar. 
| Iris Nieding \& E. Katharina Klaudy

\begin{tabular}{|c|c|c|c|c|c|c|c|c|c|c|}
\hline $\begin{array}{r}\text { 6unp!!q } \\
-\lrcorner \partial \jmath ! \partial M \text { pun }-\imath, 10 \mathrm{y}\end{array}$ & $x$ & & $\times$ & $\times$ & $\times$ & & $\times$ & & & $\times$ \\
\hline 6unpI!qsny & $\times$ & $\times$ & & $x$ & & $\times$ & & & & $\times$ \\
\hline zuәjədwoy & $x$ & & & $x$ & $\times$ & $\times$ & $\times$ & $\times$ & $\times$ & $x$ \\
\hline 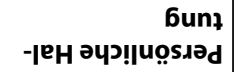 & $\times$ & & & $\times$ & & $\times$ & $\times$ & & $\times$ & $\times$ \\
\hline snyo\} גәزеuo!̣бәy & $\underset{\substack{\frac{1}{z} \\
z}}{3}$ & $\stackrel{\varrho}{\bar{\varpi}}$ & $\stackrel{\varrho}{\bar{\alpha}} \vec{\infty}$ & 岗 & $\begin{array}{l}\sum_{0} \\
\text { in } \\
i=\frac{m}{2}\end{array}$ & $\sum_{\substack{\text { on } \\
z}}^{*}$ & 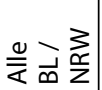 & $\Sigma$ & ঠे & 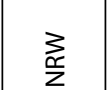 \\
\hline 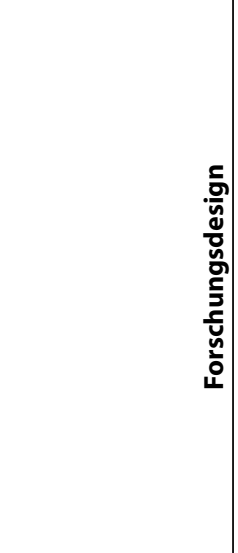 & 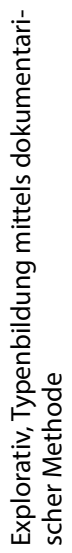 & 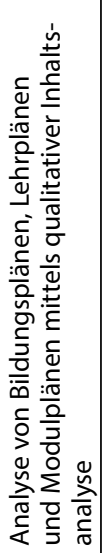 & 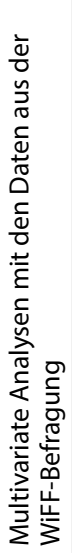 & 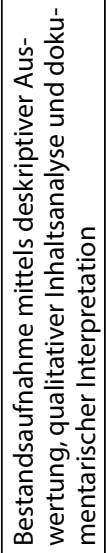 & 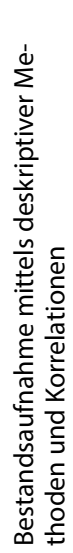 & 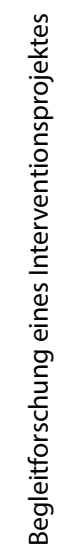 & 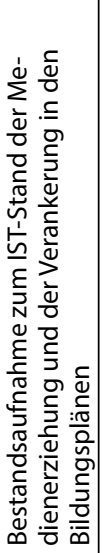 & 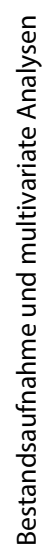 & 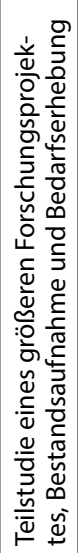 & 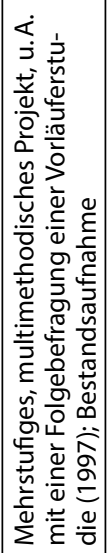 \\
\hline $\mathbf{z}$ & $\frac{\infty}{0}$ & $\Sigma$ & $\begin{array}{l}\stackrel{\omega}{\infty} \\
\stackrel{\infty}{n} \\
\end{array}$ & ผ & 8 & $\stackrel{2}{2}$ & 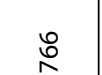 & $\overline{\tilde{N}}$ & $\infty$ & $\stackrel{2}{0}$ \\
\hline $\begin{array}{l}\frac{0}{0} \\
\vdots \\
\frac{ \pm}{0} \\
\Sigma\end{array}$ & 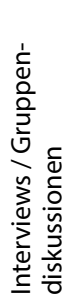 & 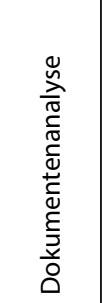 & 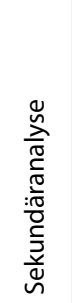 & 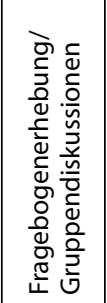 & 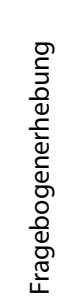 & 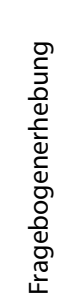 & 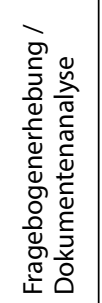 & 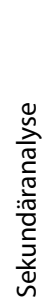 & 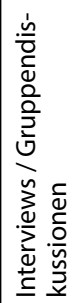 & 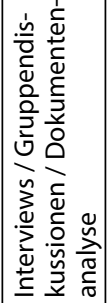 \\
\hline sрочдәW-рәх!W & & & & $x$ & & $\times$ & $\times$ & & & \\
\hline A!де?!ןeno & $\times$ & $\times$ & & & & & & & $\times$ & $\times$ \\
\hline s!pe?!zueno & & & $\times$ & & $\times$ & & & $x$ & & \\
\hline 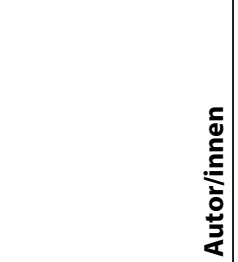 & 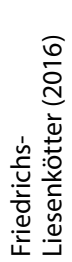 & 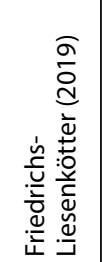 & 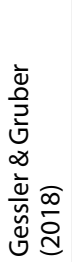 & 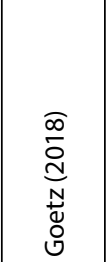 & 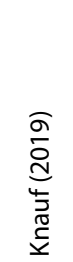 & 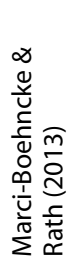 & 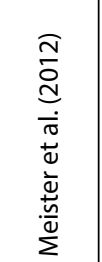 & 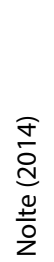 & 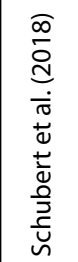 & 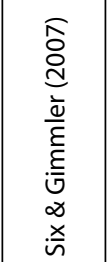 \\
\hline
\end{tabular}


Im Bereich der frühen Bildung finden sich nicht viele Studien, die pädagogische Fachkräfte mit ihren Kompetenzen und Fort- und Weiterbildungsbedarfen in den Fokus ihrer Arbeit rücken. Es scheint vielmehr so, als würden kleiner angelegte Studien im Rahmen ihrer Möglichkeiten, d.h. meist mit regionaler oder bundeslandspezifischer Eingrenzung, den „Flickenteppich“ der Forschung über Digitalisierung in Kindertageseinrichtungen weiter mit aktuellen Erkenntnissen anreichern. Wird jedoch bedacht, dass eine der Studien aus diesem Dossier bereits aus dem Jahr 2007 (Six \& Gimmler) stammt und dabei eine Nachfolgestudie zu der ersten aus dem Jahr 1997 darstellt, verwundert es, warum die Studien aus den vergangenen Jahren weiterhin einen eher explorativen Charakter aufweisen.

\section{Ergebnisse der empirischen Studien}

\subsection{Haltung der pädagogischen Fachkraft}

Im Gegensatz zur Bildungsinstitution Schule stellt der Einsatz von Computern als Bildungsmedium in Kindertageseinrichtungen eher die Ausnahme dar. Gründe dafür werden in den häufig bestehenden negativen Wirkannahmen auf die Entwicklung der Kinder gesehen. Aus Sicht von Erziehern und Erzieherinnen werden z.B. andere Bildungsbereiche als relevanter für Kinder angesehen und es besteht vielfach die Meinung, dass Kinder vor einer Überforderung durch Informationsüberflutung geschützt werden müssten. Die Vermutung, dass es durch einen erhöhten Fernsehkonsum zu einer Steigerung aggressiver Verhaltensweisen komme, stellt einen Grund für eine Ablehnung des Einsatzes von digitalen Medien in Kindertageseinrichtungen dar (Friedrichs-Liesenkötter, 2016, S. 23 f.). Andere Gründe beziehen sich auf ein begrenztes Wissen der Erzieher und Erzieherinnen im Bereich der Medienerziehung und über medienpädagogische Umsetzungsmöglichkeiten, die zu Unsicherheiten führen (Six \& Gimmler, 2007, S. 192 ff.). Die Haltung der pädagogischen Fachkräfte ist damit ein ausschlaggebender Faktor für die Umsetzung und Verankerung medienerzieherischer Inhalte in den Einrichtungen. Insgesamt sechs der zehn vorliegenden Studien berücksichtigen in ihren Analysen die Haltung und Einstellungsmuster der Erzieher und Erzieherinnen vorrangig qualitativ oder in Kombination in Form eines Mixed-Methods Ansatzes (Friedrichs-Liesenkötter, 2016; Goetz, 2018; MarciBoehncke \& Rath, 2013; Meister et al., 2012; Schubert et al., 2018; Six \& Gimmler, 2007). Dass dieser Aspekt in dem Bedingungsgefüge äußerst komplex ist, zeigt sich insbesondere in den Gruppendiskussionen und Interviews, die auch Unsicherheiten der Fachkräfte hervorheben (Friedrichs-Liesenkötter, 2016; Goetz, 2018; Schubert et al., 2018; Six \& Gimmler, 2007).

Nach Nolte (2014) existiert kein einheitlich grundlegendes Verständnis darüber, was unter Medien in der Früherziehung verstanden wird. Dies zeichnet sich bereits in der Medienforschung ab. So wird der Medienbegriff bzw. Digitalisierung 
in Kindertageseinrichtungen abhängig von den jeweiligen Forschungsfragen der Studien definiert. Als Grundproblem der Medienforschung wird z. B. betont, dass in den meisten neueren Studien unter Medien Geräte verstanden werden, die über einen Stecker oder Bildschirm verfügen. In einer Studie zur Erfassung der Medienkompetenz in niedersächsischen Kindertageseinrichtungen wird beispielsweise zwischen "neueren“ Medien (in der Studie vertreten durch Computer) und „traditionellen" Medien (Büchern) zur Strukturierung des Forschungsprozesses unterschieden (Nolte, 2014, S. 215). In der Studie zu „Mobile Medien und Internet im Kindesalter - Fokus Kindertageseinrichtungen" im Rahmen der Gesamtstudie „MoFam - Mobile Medien in der Familie“ wird über einen qualitativen Zugang die Bedeutung von digitalen Medien für den Alltag der pädagogischen Fachkräfte untersucht. Im Zentrum steht die Beantwortung der Fragen, auf welche Ressourcen die Fachkräfte bei der Umsetzung zurückgreifen können und welche Bedarfe sie äußern. Diesen Fragen wird über leitfadengestützte Interviews mit Leitungskräften und Gruppendiskussionen mit Erziehenden in 33 bayerischen Kitas nachgegangen. Innerhalb der Studie wird zwischen mobilen und digitalen Medien und dem Internet unterschieden (Schubert et al., 2018). Auch wird der Medienbegriff im Sinne eines Sammelbegriffs für apersonale Trägermedien, wie Buch, Computer oder Internet verstanden. Eine andere Definition bezieht sich nur auf elektronische Medien, und häufig wird unter digitalen Medien nur der Einsatz von Kameras, Musikplayern etc. gefasst, die im Rahmen der pädagogischen Abläufe seit Jahren selbstverständlich eingesetzt werden (Nolte, 2014, S. 214). Betont wird zudem ein mangelndes medienpädagogisches Engagement von Erziehern und Erzieherinnen, welches damit erklärt wird, dass ein erheblicher Teil der Fachkräfte unter Medienerziehung den bloßen Einsatz von Medien assoziieren (Six \& Gimmler, 2007, S. 276).

Ebenso wie zum Medienbegriff werden auch keine eindeutigen Aussagen zur generellen Haltung der Fachkräfte zum Einsatz von digitalen Medien in den Studien getroffen. Der Einsatz in der Kita ist erschwerend von einer kontroversen Diskussion über die Einführung und Sinnhaftigkeit in der frühen Bildung begleitet. Dies führt dazu, dass die Integration digitaler Medien sowohl bejubelt als auch verteufelt wird. Als Gründe werden u.a. die Einstellung der Fachkräfte für eine eher ablehnende Haltung vermutet, die von persönlichen Einstellungs- und Wissenskriterien abhänge (Marci-Boehncke \& Rath, 2013, S. 234). Ihre individuelle Perspektive ist sowohl durch selbstselektive Mechanismen als auch durch negative Meinungsbilder außerhalb der Kindertageseinrichtung gekennzeichnet. Diese erhalten häufig mehr Gewichtung als eigene Erfahrungen und wissenschaftliche Erkenntnisse (Sälzer, 2017, S. 75). Zudem äußern sich pädagogische Fachkräfte häufig über negative Aspekte des Medienumgangs in Familien. Themen sind dabei kaum aktivierende Nutzungsweisen, geringe Begleitung der Kinder bei der Mediennutzung sowie der unreflektierte Umgang mit Medien, der den Kindern vorgelebt wird (Schubert et al., 2018, S. 2). Diese Aussagen werden durch die Feststellung ergänzt, dass pädagogische Fachkräfte über kein differenziertes Bild verfügen, welche Bedeutung Medien 
in der Lebenswelt der Kinder einnehmen (Marci-Boehncke \& Rath, 2013, S. 233 ff.; Sälzer, 2017, S. 73 f.).

Darüber hinaus werden Einstellungen zu und Nutzung von digitalen Medien auch durch in eigenen Nutzungserfahrungen erworbenen Barrieren und Ängsten beeinflusst. Diese lösen sich nahezu auf, sobald positive Erfahrungen mit dem Einsatz digitaler Medien gesammelt werden. Das Projekt „Medienkompetent zum Schulübergang" hatte zum Ziel, über einen Zeitraum von 2010 bis 2014, die teilnehmenden Erzieher und Erzieherinnen regelmäßig mit ihren Einstellungen über digitale Medien zu konfrontieren, während sie zur Steigerung ihrer Medienkompetenz von Mediencoaches unterstützend begleitet wurden (Marci-Boehncke \& Rath, 2013, S. 14f.). Andere Aussagen belegen ein großes Interesse der Fachkräfte an dem Medieneinsatz in Kitas. So zeigt sich bei einer quantitativen Erhebung in 766 Einrichtungen aus Nordrhein-Westfalen, dass ein großes Interesse für die Medienerziehung vorhanden ist und sich Fachkräfte neben dem Elternhaus als verantwortlich für die Medienerziehung der Kinder sehen (Meister et al., 2012, S. 46 ff.). Dieses Ergebnis bestätigt sich im Rahmen der quantitativen Fragebogenerhebung von 104 Kitas in Berlin über Gelingensbedingungen der frühen Medienbildung, in der $60 \%$ der Erziehenden Medienerziehung als ein wichtiges Thema für ihre pädagogische Arbeit ansehen und ein Großteil der Befragten einem medienfreien "Schonraum“ ablehnend gegenübersteht (Goetz, 2018, S. 267 ff.).

Die Ergebnisse einer qualitativen Studie zur Förderung der Medienkompetenz in Kitas aus NRW belegen die unterschiedlichen Haltungen von Fachkräften: Manche Erzieher und Erzieherinnen sehen es als wichtig an, den als zu hoch und schädlich eingeschätzten Medienkonsum von Kindern durch reflektive Medienerziehung zu reduzieren. Andere wiederum lehnen digitale Medien in der Kita gänzlich ab, um den Medienkonsum durch die Schaffung eines Schonraumes zu kompensieren und wieder andere schätzen den Einsatz als wichtig ein, ohne dies weiter zu reflektieren. Nur einige wenige der befragten Erzieher und Erzieherinnen sehen eine Notwendigkeit der Medienbildung in Kindertageseinrichtungen und können diesen Förderbereich auch begründen (Six \& Gimmler, 2007, S. 282f.). Die Ergebnisse entstammen einem mehrstufigen multimethodischen Projekt, in dem insgesamt 605 pädagogische Fachkräfte über telefonische und persönliche Interviews, zum Teil erneut nach einer zehn Jahre zuvor stattgefundenen Vorläuferbefragung, über ihre Kenntnisse, Einstellungen und Problemwahrnehmungen in der medienpädagogischen Praxis sowie über Rahmenbedingungen der Ausbildung und Inanspruchnahme von Fortbildungen befragt wurden (Six \& Gimmler, 2007, S. 13 f.).

Die Haltung des pädagogischen Personals zur Medienerziehung steht folglich in einem engen Zusammenhang mit ihrem Medieneinsatz im pädagogischen Alltag, da dieser vom persönlichen Engagement der Fachkräfte geprägt ist. Die Ergebnisse einer Studie zur Medienerziehung in Kindertageseinrichtungen zeigen ebenfalls die sich wiedersprechenden Haltungen auf. Dafür wurden angehende Erzieher und Erzieherinnen aus NRW qualitativ über die eigene und die kind- 
liche Mediennutzung, ihre Schwerpunkte und Zielsetzungen, ihr Verständnis von Medienerziehung und die Umsetzung in der Praxis befragt, um daraus eine Rekonstruktion der „medienerzieherischen Habitusformationen“ der Erzieher und Erzieherinnen zu erstellen (Friedrichs-Liesenkötter, 2016, S. 133 ff.).

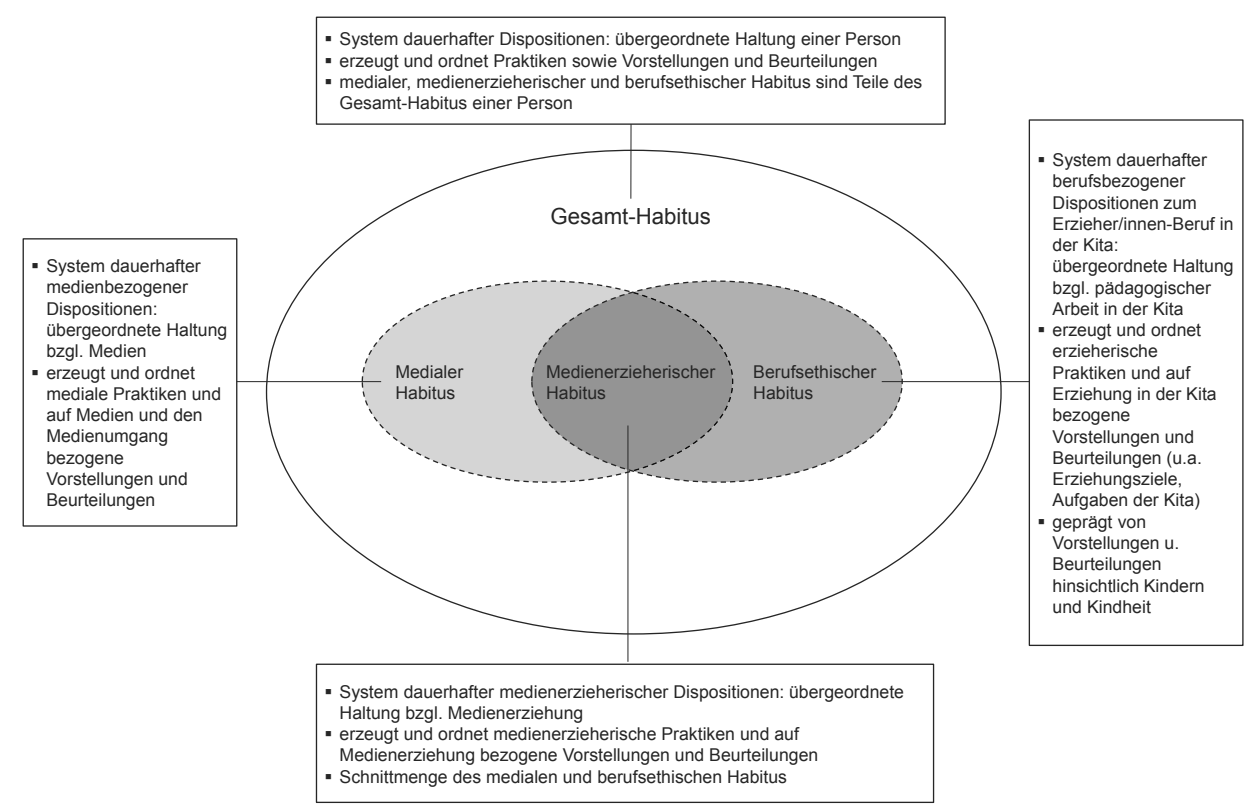

Abbildung 3: Medienerzieherischer Habitus

Quelle: Friedrichs-Liesenkötter, 2016, S. 130

Ausgehend vom Habitus-Konzept von Bourdieu werden einzelne Aspekte des Gesamt-Habitus im Kontext der Medienerziehung in Kindertagesstätten betrachtet. Differenziert wird zwischen medialen, berufsethischen und medienerzieherischen Habitus (siehe Abbildung 3). Als Ausgangspunkt für eine später erfolgte Differenzierung in zwei medienerzieherische Handlungsformen wurde die Typisierung verwendet, die die Schnittmenge des medialen (z.B. Vorstellungen und Beurteilungen bzgl. Medienwirkungen auf Kinder) und berufsethischen Habitus (berufsbezogene Dispositionen erzieherischer Praktiken sowie Vorstellungen und Beurteilungen zur erzieherischen Arbeit in der Kindertageseinrichtung) darstellt (FriedrichsLiesenkötter, 2016, S. 290). Der mediale Habitus wird dabei als ein zentraler Teil des kulturellen Kapitals von Erziehern und Erzieherinnen durch ihre medienpädagogischen Fähigkeiten geprägt (Marci-Boehnke \& Rath, 2013, S. 233 ff.). Die Ergebnisse der in dieser Studie eingesetzten komparativen Gruppendiskussion verweisen auf zwei medienerzieherische Habitustypen in den Kitas:

- Typ I unterscheidet zwischen „guten“ (z. B. Bücher, Hörspiele und Digitalkamera) und „schlechten“ (z.B. Fernseher, Computer und Spielekonsole) Medien. Um 
Kinder vor schlechten Medien zu schützen, wird die Kita als medialer Schutzraum betrachtet, da bereits eine hohe Nutzung in der Familie erfolgt (FriedrichsLiesenkötter, 2016, S. 340).

- Typ II geht ebenfalls von einer hohen Präsenz von Medien in der Familie aus, vertritt jedoch die Haltung, dass Medienerziehung mit dem Einsatz elektronischer Medien zum Aufgabenbereich der Kita zählt, um Kinder frühzeitig an eine verantwortungs- und risikobewusste Mediennutzung heranzuführen (FriedrichsLiesenkötter, 2016, S. 347).

Weitere Ergebnisse der Studie deuten darauf hin, dass ein befürwortender Habitus (Typ II) für Medienerziehung und den Einsatz elektronischer Medien keineswegs ausreiche, um medienpädagogische Aktivitäten in der Kita zu sichern. Obwohl elektronische Medien im Alltag der an der Studie beteiligten angehenden Fachkräfte eine zentrale Rolle spielen, ist die berufliche Nutzung vorrangig rezeptiv und kommunikationsorientiert und trägt nicht zu einem proaktiven Einsatz in der frühpädagogischen Praxis bei. Weiter zeigt sich z. B., dass insbesondere auf Seiten älterer Erzieher und Erzieherinnen Defizite im Umgang mit Mediengeräten bestehen und knappe Zeit- und Personalressourcen die Umsetzung pädagogischer Alltagsanforderungen und Ziele insgesamt behindern (Friedrichs-Liesenkötter, 2016, S. 353).

Projekte mit Interventionscharakter zeigen auf, dass sich der mediale Habitus durch regelmäßige Reflexionen und Konfrontation mit der eigenen Einstellung anpassen lässt. Dies sei eine effektivere Möglichkeit, nachhaltige Ergebnisse und Kompetenzsteigerungen zu erzielen als durch kurzweilige Fortbildungen, die keinen Nachklang in der pädagogischen Praxis haben (Marci-Boehncke \& Rath, 2013, S. 15). Während zu Projektbeginn noch $17 \%$ der teilnehmenden Erzieher und Erzieherinnen eine bewahrpädagogische Einstellung aufwiesen, konnte diese bei allen Personen im Verlauf des Projektes in eine aufgeschlossene Position umgewandelt werden. Als weiterer positiver Effekt lässt sich die Kompetenzsteigerung der Teilnehmenden feststellen, die sich vor Projektbeginn zu $63 \%$ sicher im Umgang mit digitalen Medien fühlten und nach Ablauf des Projektes zu 77\% (Marci-Boehncke \& Rath, 2013, S. 74).

Die Vielschichtigkeit der Erkenntnisse veranlasst Six und Gimmler (2007) zu dem Fazit, dass dem zugrunde gelegten Verständnis von Medienerziehung eine Schlüsselrolle für die Umsetzung und Anwendung zukommt. Einstellung und Umfang der diesbezüglichen Aktivitäten von Fachkräften „hängt in erheblichem Maße davon ab, - was sie unter Medienerziehung verstehen, - welche individuelle Vorstellung sie davon haben, was Medienerziehung auf der operationalen Ebene ist oder bedeuten kann und - unter welchen Prämissen sie welche Ziele mit Medienerziehung assoziieren." (Six \& Gimmler, 2007, S. 283) 


\subsection{Kompetenzen des pädagogischen Personals}

Eine möglichst umfassende Medienkompetenz wird als Schlüsselkompetenz für gesellschaftliche Teilhabe im Sinne von Partizipation, sowie zur Bewältigung z. B. schulischer, beruflicher und privater Anforderungen angesehen (Fthenakis \& Walbiner, 2018). Ein Problem der wissenschaftlichen Forschung besteht darin, dass sich in den vergangenen Jahren verschiedene Definitionen zum Begriff Medienkompetenz entwickelt haben. Fthenakis \& Walbiner stellen eine grundlegende Übersicht zusammen, wie digitale Kompetenz zu fassen sei:

- als „neuer Begriff zur Beschreibung technologiebezogener Fertigkeiten“, der sich gemäß der technologischen Transformation stetig weiterentwickeln wird (Fthenakis \& Walbiner, 2018, S. 54)

- oder im Sinne von „Digital Literacy“, als „Fertigkeiten, Wissen und Einstellungen beim Gebrauch digitaler Medien, um befähigt zu sein, die Herausforderungen in der lernenden Gesellschaft zu bewältigen" (Fthenakis \& Walbiner, 2018, S. 55) und auch „die Auswirkungen der digitalen Technologien auf die Gesellschaft zu verstehen und $\mathrm{zu}$ erkennen, wie sich digitale von traditioneller Kommunikation unterscheidet" (Fthenakis \& Walbiner, 2018, S. 90).

Die medienpädagogische Kompetenz von Erziehern und Erzieherinnen ist ausschlaggebend dafür, in welchem Maße eine konstruktive Auseinandersetzung mit dem Medienalltag von Kindern zentraler Bestandteil der Kita-Arbeit in Kindertageseinrichtungen wird. Der Stand der Forschung hinsichtlich der Kompetenzen des pädagogischen Personals wird als Forschungsdesiderat bezeichnet (Graube et al., 2015, S. 134).

Eine weitere Lücke in der Forschungslandschaft stellen Kompetenzmodelle für pädagogische Fachkräfte im Elementarbereich dar, die sich auf Voraussetzungen für eine medienpädagogische Praxis beziehen. Bergner et al. (2018) versuchen dem mit theoretischen Überlegungen entgegenzuwirken, indem sie ein generisches Modell der professionellen Kompetenz von Lehrkräften (Kunter \& Baumert, 2011) nutzen und es an die Rahmenbedingungen von Kindertageseinrichtungen anpassen. Diese Überlegungen basieren auf Ansätzen aus unterschiedlichen Bundesländern und Praxisprojekten wie der Stiftung „Haus der kleinen Forscher“, die zum Ziel haben, Kinder im Rahmen der frühen Bildung in den MINT-Bildungsbereichen zu stärken und zu einem nachhaltigen Handeln zu befähigen (Bergner et al., 2018, S. 166 f.). Die jüngste quantitative Fragebogenstudie mit 190 Kitas deutet darauf hin, dass eine professionelle Medienkompetenz in den Einrichtungsteams noch nicht vorhanden ist. Etwa ein Drittel der befragten Leitungen stuft die Kompetenzen der Fachkräfte für den Medienumgang als gering oder nicht vorhanden ein (Knauf, 2019, S. 17).

Im Hinblick auf das eigene Vermögen, Kindern Medienkompetenz zu vermitteln, wird oftmals auf die eigene Selbstwirksamkeitserwartung der Erzieher und Erzieherinnen verwiesen. Unter Selbstwirksamkeit wird die subjektive Bewertung 
der befragten Erzieher und Erzieherinnen bezüglich ihrer „Einschätzung, mit dem eigenen Erziehungshandeln etwas bei den Kindern bewirken zu können" verstanden (Six \& Gimmler, 2007, S. 114). Eine schlechte Selbsteinschätzung geht dabei häufig mit einer niedrig eingeschätzten eigenen Medien- sowie Technikkompetenzen einher (Graube et al., 2015, S. 44 f.; Marci-Boehncke \& Rath, 2013, S. 68). Die subjektive Bewertung der Befragten bezüglich ihrer Selbstwirksamkeit zu Bedingungen und Handlungsformen der Medienerziehung fiel im Durchschnitt nicht optimistisch aus: Ein großer Teil glaubt nicht daran, einen negativen Medienumgang der Kinder mittels der eigenen Kompetenzen verbessern zu können (Six \& Gimmler, 2007, S. $255 \mathrm{ff}$.). Zu einer Stärkung der Selbstwirksamkeitserwartung bei jüngeren Fachkräften kann ein einrichtungs- oder trägerspezifisches Konzept zu mehr Sicherheit im Umgang mit Medien beitragen, was durch geeignete Vorbilder in der Einrichtung weiter gefördert werden kann (Goetz, 2018, S. 314).

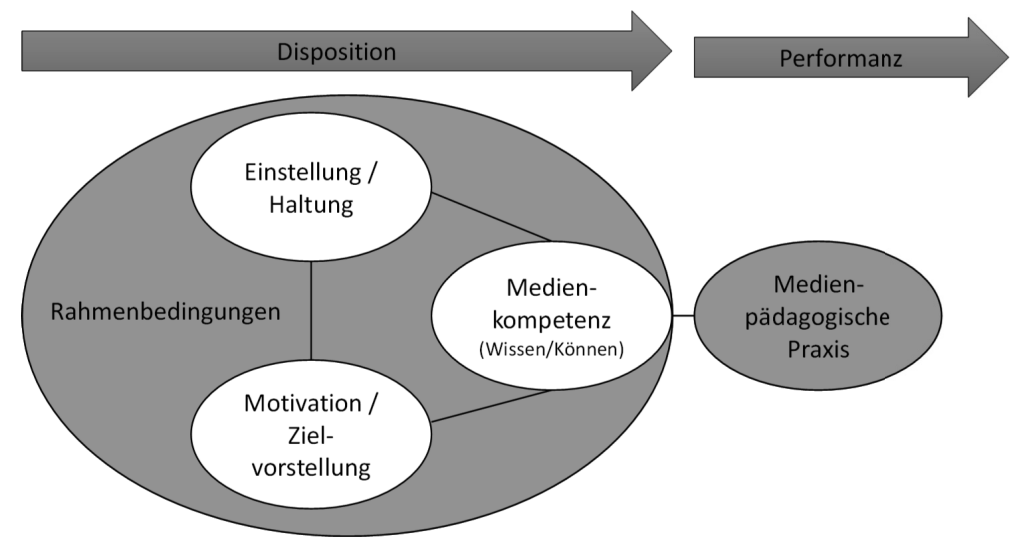

Abbildung 4: Bedingungsgefüge medienpädagogischer Praxis

Quelle: eigene Darstellung nach Nolte, 2014, S. 216

In einer 2012 durchgeführten Fragebogenstudie wurden 67 Kitas aus Niedersachsen $\mathrm{zu}$ den Bedingungen ihrer medienpädagogischen Praxis befragt. Erfasst wurde eine Selbsteinschätzung der Fachkräfte mit Fokus auf ihr Wissen und Können bei ihrer eigenen Computernutzung. Die Ergebnisse wurden in ein Bedingungsgefüge (siehe Abbildung 4) mit Einflussfaktoren auf die medienpädagogische Praxis eingefügt (Nolte, 2014, S. 215). Welche verschiedenen Faktoren einen Einfluss auf die tatsächliche medienpädagogische Praxis ausüben, wird mittels eines komplexitätsreduzierten Kompetenzmodells abgebildet, das dem gängigen Kompetenzmodell für pädagogische Fachkräfte nach Fröhlich-Gildhoff, Nentwig-Gesemann und Pietsch (2011, S. 17) folgt. Die medienpädagogische Kompetenz, dargestellt als Disposition (Rahmenbedingungen sowie die Kombination aus Haltung, Medienkompetenz, Motivation und Zielvorstellung), äußert sich in der tatsächlich erbrachten Leistung der medienpädagogischen Praxis (Performanz). Es zeigte sich, dass je kompetenter 
sich die Fachkräfte im Umgang mit Computern einschätzten, diese auch umso häufiger in der medienpädagogischen Arbeit zum Einsatz kommen. Computerbezogene Kompetenzen hatten somit einen deutlich direkten Einfluss auf die Mediennutzung in der medienpädagogischen Praxis der Fachkräfte (Goetz, 2018, S. 186; Nolte, 2014, S. 218 f.). Vergleichbare Ergebnisse zur Selbsteinschätzung betreffen auch Leitungskräfte. Bei der Einschätzung zum eigenen Medienhandeln stehen die Leitungskräfte digitalen Medien jedoch aufgeschlossener gegenüber, zeigen mehr Selbstvertrauen im Umgang mit Medien und messen ihnen eine größere Bedeutung für die pädagogische Praxis zu. Auch fühlen sie sich nicht oder selten vom technischen Fortschritt überfordert (Goetz, 2018, S. 191; Meister et al., 2012, S. 46 ff.).

Die Darlegungen verweisen auf die Relevanz eines umfassenden Verständnisses von Medien sowie auf die Notwendigkeit in der beruflichen Praxis mit ihnen umzugehen. Im Kontext des Medieneinsatzes bilden Schubert et al. (2018) die Medienkompetenz von pädagogischen Fachkräften über drei Idealformen des situationsorientieren Medieneinsatzes ab, wobei sie sich an den Ausführungen von Friedrichs-Liesenkötter (2016) orientieren (siehe Abbildung 5).

\begin{tabular}{|l|}
\hline $\begin{array}{l}\text { Handlungs- / produktions- } \\
\text { orientierte Medienarbeit }\end{array}$ \\
\hline - eigenständiges Schaffen \\
von Medienprodukten \\
- Medien als Gestaltungs- \\
und Ausdrucksmittel \\
- z.B. einen Trickfilm pro- \\
duzieren \\
\hline
\end{tabular}

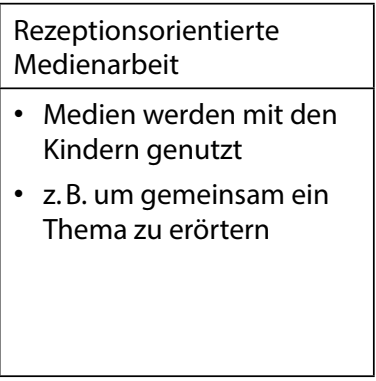

Abbildung 5: Idealtypische Formen der Medienerziehung Quelle: eigene Darstellung, nach Friedrichs-Liesenkötter, 2016, S. $73 \mathrm{ff}$.

\begin{tabular}{|l|}
\hline $\begin{array}{l}\text { Reproduktionsorientierte / } \\
\text { reflexive Medienarbeit }\end{array}$ \\
\hline - kein Medieneinsatz nötig \\
- Aufgreifen und reflexives \\
Aufarbeiten von Medien- \\
erlebnissen der Kinder \\
- z.B. medienbezogene \\
Gespräche, Rollenspiele, \\
gemalte Bilder
\end{tabular}

Reproduktionsorientierte / reflexive Medienarbeit

- kein Medieneinsatz nötig Aufarbeiten von Mediender Kinder Gespräche, Rollenspiele

Im Sinne der handlungs- und produktionsorientierten Medienarbeit werden Medien vor allem für Aktionen eingesetzt, die sich z. B. mit Fotografieren, Malen und Filmaufnahmen beschäftigen. Eine rezeptionsorientierte Medienarbeit gemeinsam mit den Kindern, z. B. Ansehen von Inhalten auf Computern oder Laptops, findet selten statt. Eine klassische rezeptionsorientierte Arbeit in der pädagogischen Praxis ist das Anhören und Mitsingen oder Tanzen von Hörspielen und Musik. Dies erfolgt nicht mehr nur über traditionelle Medien wie CDs, sondern vermehrt auch über Musik- und Videoportale, wie bspw. YouTube. Auch Elemente einer reproduktionsorientierten und reflexiven Medienarbeit konnten in den befragten Einrichtungen ermittelt werden. Dabei geht es vor allem um das Sprechen mit Kindern über Medienerlebnisse oder das Einbauen defekter Geräte (z.B. Mobiltelefone oder Tastaturen) in das Spiel der Kinder, wodurch eine Verknüpfung der digitalen und der analogen Welt hergestellt wird (Schubert et al., 2018, S. 22 ff.). Die Ergebnisse dieser Studie verweisen darauf, dass Kombinationen der Arten von Medienerziehung 
häufiger vorkommen als die oben abgebildeten Reinformen. So ist die rezeptionsorientierte Medienarbeit z.B. häufig mit einem kommunikativen Austausch und digital vermittelte Informationen mit realen Rahmenbedingungen aus dem eigenen Lebenskontext verknüpft. Ergebnisse weiterer Studien verweisen ebenfalls darauf, dass rezeptive Kompetenzen stärker ausgeprägt sind als vermittlungsorientierte (Marci-Boehncke \& Rath, 2013, S. 233 ff.).

Der Einfluss medienpädagogischer Fortbildungen sowie der medienpädagogische Ausbildungsanteil zeigten keinen direkten Einfluss auf das Nutzungsverhalten in der Praxis, im Gegensatz zum Einfluss von Berufserfahrung (Nolte, 2014, S. 218). Ausgehend von der Annahme, dass eine längere Berufserfahrung zu einem sicheren Umgang mit schwierigen, pädagogischen Themen führt, wenn diese als relevant angesehen werden, lässt sich diese auch auf die medienpädagogische Praxis übertragen. Wenn der Medieneinsatz als wichtig angesehen wird, findet er nicht nur vermehrt statt, sondern auch entgegen kritischer Stimmen (Nolte, 2014, S. 219). Diese Aussage steht im Widerspruch zu den Ergebnissen, die einen negativen Einfluss des Alters der Erzieher und Erzieherinnen auf den Medieneinsatz sehen. Der Faktor Berufserfahrung sollte in zukünftigen Studien stärker berücksichtigt werden, um diese Zusammenhänge weiter aufzudecken.

\subsection{Aus- und Fortbildung im Kontext der (Aus-)Bildungspläne}

Frühpädagogische Fachkräfte gelten in der Praxis als eine Gruppe mit einer besonders hohen Weiterbildungsaffinität (Buschle \& König, 2018). Dies können auch Teilnahmequoten an berufsbezogenen Weiterbildungen belegen. Laut Gessler und Gruber (2018, S. 156) liegt deren Quote bei 85,8\%, während sie bei allen Erwerbstätigen in Deutschland bei $56 \%$ liegt (Bilger, Behringer, Kuper \& Schrader, 2017). Dadurch kann davon ausgegangen werden, dass pädagogische Fachkräfte ein großes Interesse daran haben, ihre Arbeitsinhalte und -prozesse gemäß aktuellen Entwicklungen umzusetzen. Anders als bei anderen Berufsgruppen hängt die Weiterbildungsbereitschaft nicht von den soziodemografischen Merkmalen der Fachkraft ab, sondern von ihren persönlichen Einstellungen zu der Wichtigkeit von Fortbildungen bzw. von spezifischen Inhalten (Gessler \& Gruber, 2018). Den Nachweis konnten die Autorinnen basierend auf einer großen Datenlage (1.585 Einrichtungsleitungen und Mitarbeitende aus der WiFF-Studie) mittels binär logistischer Regressionen erbringen, indem sie Einflussfaktoren auf die Weiterbildungschance von frühpädagogischen Fachkräften errechneten. Ausschlaggebend ist laut diesen Untersuchungsergebnissen auch der Grad der Informiertheit der Fachkräfte. Das Wissen über Fortbildungsangebote oder über bestimmte Portale, die Fortbildungsangebote für spezielle Bereiche auflisten, erhöht die Chance an einer Teilnahme um ein Vielfaches. Dies setzt die Bereitschaft voraus, aktiv nach diesen Angeboten zu suchen. Die Autorinnen merken dazu an, dass dieses Ergebnis 
für Trägerverantwortliche von großem Interesse sein kann, da sich hier ein Gestaltungsspielraum eröffnet, Informationen über Fortbildungsangebote transparent an die Mitarbeitenden in den Einrichtungen weiterzugeben (Gessler \& Gruber, 2018, S. 157).

\subsubsection{Ausbildung und Studium}

Das medienerzieherische Handeln wird maßgeblich durch die Einstellungen und Kompetenzen der Fachkräfte bestimmt (vgl. Kapitel 3.2). Eine zentrale Stellschraube für Einstellungsmuster und Kompetenzen ist die erzieherische Ausbildung: „Wer bereits in der Ausbildung medienpädagogisch relevante Kenntnisse, Fähigkeiten und Fertigkeiten erworben hat und dabei auch erfahren hat, was Medienerziehung umfasst und weshalb sie notwendig ist, wird später mit anderen Voraussetzungen und vermutlich in entsprechend anderer Weise Medienerziehung praktizieren." (Six \& Gimmler, 2007, S. 30) Da in Deutschland für den Bereich der Elementarbildung kein einheitlich vorgeschriebener Lehrplan existiert, an den sich alle Institutionen und Beteiligte orientieren müssen, kann die inhaltliche Ausrichtung einer Ausbildung je nach Berufsschule und Curricula stark variieren. Der länderübergreifende Lehrplan aus dem Jahr 2012 beinhaltet Angaben über das Berufsbild der Erziehenden sowie Anforderungen an ein kompetenzorientiertes Qualifikationsprofil. Es stellt eine gestalterische Orientierungshilfe für die Bundesländer und Fachschulen dar, ist jedoch keine verbindliche Vorgabe in der Auslegung der einzelnen Lernfelder (KMK, 2012). Dieser Lehrplan begreift die Vermittlung von Medienkompetenz als eine Querschnittsaufgabe innerhalb von fünf Aufgaben, die durch den gesellschaftlichen Wandel an Bedeutung gewonnen haben. Darin heißt es, „sozialpädagogische Fachkräfte unterstützen Kinder, Jugendliche und junge Erwachsene bei der Entwicklung ihrer Medienkompetenz" (KMK, 2012, S. 5).

Friedrichs-Liesenkötter (2019) betrachtet in einer Dokumentenanalyse den länderübergreifenden Lehrplan, die bundeslandspezifischen Lehrpläne für die fachschulische Ausbildung, die Modulpläne für frühkindliche Studiengänge sowie die 16 Bildungspläne der Bundesländer. Sie geht damit der Frage nach, welchen Stellenwert Medienerziehung oder Medienbildung in diesen Dokumenten einnehmen, die maßgeblichen Einfluss auf die medienpädagogische Praxis ausüben (FriedrichsLiesenkötter, 2019, S. 5 f.). Die Analyse der fachschulischen Ausbildungspläne zeigt zwar eine Orientierung an dem länderübergreifenden Lehrplan und der darin beschriebenen Medienkompetenz, jedoch in unterschiedlicher Ausdifferenzierung und Verankerung in den Curricula. Zum Teil werden in den Ausbildungsplänen die groben Vorgaben zur Querschnittsaufgabe Medienerziehung wortgetreu übernommen, wodurch ein Defizit an konkreten Ausführungen, Beispielen und Umsetzungsmöglichkeiten entsteht (Friedrichs-Liesenkötter, 2019, S. 19 f.). 
Das Erlernen medienpädagogischer und -erzieherischer Inhalte innerhalb der Ausbildung bildet die Hauptfragestellung mehrerer Studien, deren sowohl quantitativen als auch qualitativen Ergebnisse sich stark ähneln (Friedrichs-Liesenkötter, 2016; Goetz, 2018; Marci-Boehncke \& Rath, 2013; Six \& Gimmler, 2007). In den meisten Fällen wird die Ausbildungssituation im Rahmen einer Fragebogenerhebung ermittelt, die sich vornehmlich auf das reine Vorhandensein medienerzieherischer oder medienpädagogischer Inhalte konzentriert. So zeigt sich, dass bei $53 \%$ der befragten Erzieher und Erzieherinnen aus Berliner Kitas jenseits der Leseförderung keine Inhalte der Medienerziehung während der Ausbildung thematisiert wurden (Goetz, 2018, S. 254). Friedrichs-Liesenkötter (2016) erfasst medienpädagogische Inhalte mit Hilfe qualitativer Methoden in Form von Interviews und Gruppendiskussionen angehender Erzieher und Erzieherinnen und analysiert so die Ausbildungsinhalte an Fachschulen des Sozialwesens in NRW. Durch diese Kombination konnte sie herausfinden, dass zwar medienpädagogische Inhalte in den Ausbildungsplänen verankert sind, diese in der Realität aufgrund mangelnder Zeitressourcen aber nur theoretisch behandelt werden, ohne diese praktisch einzuüben (Friedrichs-Liesenkötter, 2016, S. 357). Damit fehlen den Fachkräften nach der Ausbildung das Wissen über Handlungs- und Umsetzungsmöglichkeiten in der pädagogischen Praxis sowie konkrete Erfahrungen mit der Durchführung medienpädagogischer Aktivitäten (Six \& Gimmler, 2007, S. 279f.).

Im Kohortenvergleich zeigt sich, dass medienerzieherische Inhalte stärker in der heutigen Ausbildung thematisiert werden. 77,8\% der Fachkräfte mit über 30 Jahren Berufserfahrung hatten keine medienpädagogischen Ausbildungsinhalte, während es bei jüngeren Fachkräften mit ein bis fünf Jahren Berufserfahrung 15\% waren (Marci-Boehncke \& Rath, 2013, S. 71). Auch bei Goetz (2018) sind unter den $47 \%$ Erziehern und Erzieherinnen mit medienpädagogischen Inhalten in der Ausbildung vermehrt jüngere Mitarbeitende vertreten (Marci-Boehncke \& Rath, 2013, S. 254). Dies bestätigen auch die Ergebnisse von Six und Gimmler aus dem Jahr 2007 (S. 278f.) Die Ausbildungsinhalte scheinen sich den gesellschaftlichen Anforderungen entsprechend in den vergangenen Jahren erweitert zu haben. Auch von den ausbildenden Lehrkräften wird die Medienpädagogik stärker thematisiert. Bedenklich ist jedoch der festgestellte fehlende Zusammenhang zwischen ausgebildeten Medienkompetenzen und der Umsetzung in die Alltagspraxis der Einrichtungen. Es lassen sich folglich Hinweise darauf finden, dass eine theoretische Ausbildung kein Garant für eine medienpädagogische Praxis darstellt (Six \& Gimmler, 2007, S. 278f.).

Auch in den Studiengängen der frühkindlichen Bildung oder Kindheitspädagogik herrscht eine hohe Diversität hinsichtlich der Verankerung medienpädagogischer Inhalte. In $60 \%$ der Studiengänge sind sie nicht vorhanden oder nur randständig als Querschnittsthema integriert. Medienpädagogische Module, die für alle Studierenden verpflichtend belegt werden müssen, finden sich in $21 \%$ der Studiengangspläne wieder und in weiteren $14 \%$ tauchen sie als Wahlfach zum obligatorischen Besuch auf (Friedrichs-Liesenkötter, 2019, S. 24). 


\subsubsection{Fort- und Weiterbildung}

Fortbildungsmaßnahmen können in der Praxis dazu eingesetzt werden, fehlende medienpädagogische Inhalte in der Ausbildung oder mangelnde Medienkompetenzen sowie Unsicherheiten der Fachkräfte auszugleichen oder vorhandene Ansätze in der Praxis zu verfestigen. Die Studien von Gessler und Gruber (2018), Goetz (2018), Knauf (2019), Meister et al. (2012) und Six und Gimmler (2007) beschäftigen sich mit Fragestellungen rund um die Nutzung von Fortbildungsangeboten oder -inhalten. Gessler und Gruber (2018) nutzen die Daten der WiFF-Fachkräftebefragung aus dem Jahr 2016, um anhand einer binär logistischen Regression die Einflussfaktoren auf die Weiterbildungschance von pädagogischen Fachkräften zu analysieren. Durch die große Datengrundlage $(\mathrm{N}=1.585)$ sind sowohl Leitungen als auch Mitarbeitende in der Studie abgedeckt und es können Aussagen über das Weiterbildungsverhalten der Erzieher und Erzieherinnen in ganz Deutschland getroffen werden. Goetz (2018) berücksichtigt in der Befragung ebenfalls insgesamt 795 Leitungs- sowie Fachkräfte, evaluiert jedoch mit einem Mixed-Methods Forschungsdesign Gelingensbedingungen für eine erfolgreiche Umsetzung von Medienbildung in Berliner Kitas. Die quantitative Fragebogenerhebung von Knauf (2019) nimmt die Bundesländer Sachsen, Baden-Württemberg und NRW in den Blick und befragte 190 Kitas zu nötigen Rahmenbedingungen für die Umsetzung von Digitalisierungskonzepten sowie zum aktuellen Stand zur Nutzung digitaler Medien in der Einrichtung aus Sicht der Leitungskräfte. Die Umsetzung von Medienerziehung in Kitas untersuchen Meister und Friedrichs-Liesenkötter (2012) für das Bundesland Nordrhein-Westfalen anhand von 766 ausgefüllten Fragebögen von Einrichtungsleitungen. In einer älteren Studie werden neben verschiedenen Fragestellungen zur Ausbildungssituation und den Rahmenbedingungen der Einrichtungen auch die Inanspruchnahme bzw. Kenntnis von und über Fortbildungen in NRW behandelt (Six \& Gimmler, 2007). Zugrunde gelegt werden dabei die qualitativen Daten aus 605 Interviews mit Erziehern und Erzieherinnen.

Formate von Fortbildungskonzepten fallen im Bereich der frühen Bildung sehr unterschiedlich aus. Es gibt Präsenzformen über ein bis mehrere Tage, InHouse Schulungen, an denen das gesamte Einrichtungsteam teilnimmt oder geförderte (Interventions- oder Modell-) Projekte, die über einen längeren Zeitraum Schulungsangebote in Einrichtungen durchführen und diese evaluieren. Webbasierte Fortbildungsmöglichkeiten wie E-Learning oder Blended Learning spielen für Fachkräfte in der frühen Bildung und in der Forschung bislang eine untergeordnete Rolle (Buschle \& König, 2018, S. 52). Die geringe Bedeutung lässt sich - ähnlich wie bei den Weiterbildungsformaten in Präsenzform - auf fehlende Transparenz und Wissen über diese Möglichkeit zurückführen, obwohl diese Formate das Argument des Personalmangels als Begründung für eine niedrige Weiterbildungsbeteiligung aushebeln könnten (Buschle \& König, 2018, S. 66). 
In der Fragebogenerhebung von Goetz (2018) geben die Leitungskräfte an, Fortbildungen als einen wichtigen und notwendigen Gegenstand für die Arbeit in Kitas anzusehen. 63 dieser Leitungskräfte sehen dabei für die Medienbildung in der Einrichtung einen Fortbildungsbedarf - vor allem hinsichtlich der Nutzung und des Umgangs mit bestimmten digitalen Medien wie bspw. Fotografie, Präsentationen oder Laptops, Computer und Internetnutzung. Fragen zur Medienerziehung spielen dabei eine untergeordnete Rolle. Dementsprechend geben auch $75 \%$ an, dass Fortbildungen und Fachtagungen wichtige Maßnahmen zur Sicherung der medienpädagogischen Kompetenz im Team seien. In $52 \%$ der Fälle werden die Inhalte in Teamsitzungen oder Dienstbesprechungen gemeinsam ausgewertet (Goetz, 2018, S. 219 ff.). Von Seiten der Erziehenden haben $40 \%$ bereits an Fortbildungen zu medienpädagogischen Themen teilgenommen (ebd., S. 257). Dieser Anteil ist etwas höher als in der Studie von Six und Gimmler (2007), in der $85 \%$ der Fachkräfte in den fünf Jahren vor der Befragung keine medienpädagogischen Fortbildungen besuchten (Six \& Gimmler, 2007, S. 277).

Die Studien stellen eine klare Schlüsselrolle der Einrichtungsleitungen heraus (Goetz, 2018, 240). Die Teilnahme an Fort- und Weiterbildungen wird nicht nur von dem Engagement der Leitungen und Fachkräfte bestimmt, sondern auch von den Vorkenntnissen über medienpädagogische Themen im Laufe der Ausbildung. Einige Studien belegen, dass dies eine Affinität für die Wahrnehmung medienpädagogischer Fortbildungen hervorruft. In der wissenschaftlichen Forschung wird diesbezüglich von der „Heilung der Gesunden“ gesprochen: Fortbildungsangebote und Projekte erreichen in der Praxis nicht jene pädagogischen Fachkräfte, die besonders von diesen Angeboten profitieren würden, sondern vermehrt jene, die bereits medienpädagogische Kompetenzen und ein Interesse an den Inhalten aufweisen (Six \& Gimmler, 2007, S. 276; Marci-Boehncke \& Rath, 2013, S. 75 f.). Dies zeigt sich insbesondere in der Differenz der Anteile der Erzieher und Erzieherinnen, die allgemein Interesse an medienpädagogischen Inhalten und Umsetzungen äußern (84\%) und derjenigen, die diese Aussage tätigen und gleichzeitig entsprechende Fortbildungsangebote in Anspruch nehmen (95\%) (Marci-Boehncke \& Rath, 2013, S. 77).

Als Herausforderungen für die Fortbildungen werden die schnelle Transformation digitaler Medien und die daraus resultierende Notwendigkeit einer reflektiven Nachverfolgung durch passende Angebote genannt (Goetz, 2018, S. 243). Fortbildungen können nicht greifen, solange Ausstattungsfragen die Umsetzung von Medienerziehung hemmen und dadurch das Verständnis von Medienerziehung auf handlungs- und produktionsorientierte Medienarbeit beschränken. Ein Umdenken hin zu reproduktionsorientierter und reflexiver Medienarbeit ohne den Einsatz physischer Geräte senkt die Barrieren deutlich (Goetz, 2018, S. 318). Diese Tendenz lässt sich unter anderem durch die Teilnahme an Projekten wie bspw. dem Modellprojekt der Landesanstalt für Medien Nordrhein-Westfalen (LfM) nachweisen, bei dem durch den Einsatz von Medien Coaches eine deutliche Haltungsveränderung bei den Teilnehmenden erzielt werden konnte (Eder et al., 2013, S. 13). 
Abschließend ist erneut auf die Rolle der Träger, Leitungen und der Fortbildungsanbietenden hinzuweisen. Ein transparenter Informationsfluss über Fortbildungsmöglichkeiten, Informationsportale und Anbietende ist ein wesentlicher Faktor, um die Teilnahme an den Angeboten zu erhöhen. Fortbildungen zu medienpädagogischen Inhalten werden deutlich seltener besucht als zu anderen pädagogische Themen, was zum Teil auf die mangelnde Transparenz zurückgeführt werden kann. Für Träger und Kitaverantwortliche ergibt sich daraus ein Ansatzpunkt dafür, solche Angebote mehr in den Blick der Fachkräfte zu rücken. Eine unterstützende und aufgeschlossene Haltung gegenüber der Bedeutung von Medienbildung in der Kita seitens der Leitung kann unter diesen Voraussetzungen eine förderliche Wirkung entfalten.

\subsubsection{Länderspezifische Bildungspläne}

Andere Untersuchungen analysieren die Verankerung von medienpädagogischen Inhalten in den Bildungsplänen der Bundesländer in Deutschland (Meister \& Friedrichs-Liesenkötter, 2012; Friedrichs-Liesenkötter, 2019). Der Vergleich zeigt, dass Medienerziehung in den meisten Bundesländern - in unterschiedlicher Gewichtung - Beachtung findet. Während 2012 (Meister \& Friedrichs-Liesenkötter) nur drei Mal ein eigener Bildungsbereich „Medien“ existierte und acht Bundesländer den Bereich im Rahmen von Kommunikation, Schrift und Sprache behandelten, hebt Friedrichs-Liesenkötter in ihrer Dokumentenanalyse von 2019 zehn Bundesländer hervor, die Medien mittlerweile als expliziten Bildungsbereich benennen. Dazu zählen Hessen, Nordrhein-Westfalen, Rheinland-Pfalz, Thüringen und Bayern sowie Berlin, Hamburg, Schleswig-Holstein, Saarland und Sachsen, wobei die letzten fünf genannten den Bereich mit anderen Bildungsbereichen verknüpfen. In den vier Bundesländern Baden-Württemberg, Mecklenburg-Vorpommern, Niedersachsen sowie Bremen werden Medien dagegen unter andere Bereiche, wie bspw. Sprache, subsummiert und finden dort eine randständige Erwähnung. In Sachsen-Anhalt und Brandenburg finden Medien in den Bildungsplänen keine Berücksichtigung. Das Hervorheben von Medienbildung als eigenen Bildungsbereich ist zunächst positiv $\mathrm{zu}$ bewerten und lässt erste Rückschlüsse darauf zu, welchen Stellenwert Medien in den Bildungsplänen der Länder einnehmen. Die Autorin weist aber ausdrücklich darauf hin, dass die inhaltliche Ausrichtung sehr divers sei (Friedrichs-Liesenkötter, 2019, S. 8). Dies hat eine nähere Untersuchung des vermittelten Medienbildes in Bildungsplänen in drei Kategorien zur Folge: Es werden erstens Chancen und Risiken mit Blick auf das Bildungspotenzial von Medien fokussiert und Medien werden als expliziter Bildungsbereich benannt, es werden zweitens eher ablehnende Haltungen in den Bildungsplänen vertreten ohne weiter auf deren Inhalte einzugehen oder das Thema Medien wird drittens nicht benannt (Friedrichs-Liesenkötter, 2019, S. 10 ff.). 
Während in der wissenschaftlichen Debatte oftmals die Haltung der Fachkräfte und Leitungen fokussiert wird, sollte hinsichtlich der Vorgaben (in Form von Bildungsplänen) auch die politische Haltung und Ausrichtung der Länder und verantwortlichen Politikern und Politikerinnen berücksichtigt werden. Sie ist ausschlaggebend und richtungsweisend für den Kurs, den Bildungsinstitutionen einschlagen und der Gewichtung der Medienpädagogik/-erziehung in den Ausbildungsplänen. Die Bildungspläne der einzelnen Bundesländer sind ein ausschlaggebender Faktor dafür, wie sich Haltungen und Kompetenzen der Fachkräfte hinsichtlich digitaler Medien entwickeln und in der pädagogischen Praxis ausgestaltet werden. In Ermangelung positiver Erfahrungen und Wissen über den Einsatz digitaler Medien in Kombination mit anderen Aktivitäten, wird Medienerziehung von pädagogischen Fachkräften als zeitliche Konkurrenz zu anderen Bildungsbereichen wahrgenommen, die in dem Wertemuster der Fachkräfte einen höheren Stellenwert einnehmen (Friedrichs-Liesenkötter, 2016, S. 354 f.). Eine künftige Aufgabe besteht darin, durch praktische Erfahrungen Möglichkeiten aufzuzeigen, wie Medienerziehung als Querschnittsaufgabe vollzogen werden kann, ohne dass Konkurrenzen zu anderen Bildungsbereichen entstehen, sondern diese vielmehr durch digitale Medien unterstützend vermittelt werden.

\section{Fazit}

Die Forschungslandschaft im Bereich der frühen Bildung zum Umgang mit digitalen Medien verweist auf ein gespaltenes Bild in der Praxis. Wie der Titel des vorliegenden Dossiers bereits besagt, bewegt sich dieses zwischen einer bewahrpädagogischen Haltung zur Schaffung eines Schutzraums und der Förderung einer reflexiven Mediennutzung für Kinder in einer durch die Digitalisierung geprägten Gesellschaft, in der Medienkompetenz als „neue“ Schlüsselkompetenz gilt. Die Ergebnisse der vorgelegten Studien haben gemeinsam, dass die medienpädagogische Praxis von unterschiedlichen, stark ineinander verwobenen Aspekten abhängt. Als besonders starke Faktoren gelten die Haltung der Fachkräfte und ihre Kompetenz im Umgang mit digitalen Medien, die durch ein Bedingungsgefüge schwer voneinander zu trennen sind. Im Zusammenspiel von Haltung und Kompetenz liefern die Studien Hinweise darauf, dass die Förderung der medienpädagogischen Praxis in Kindertageseinrichtungen an beiden Faktoren gleichermaßen ansetzen muss. Modell- und Interventionsprojekte lieferten dafür wichtige Anhaltspunkte. Auch zeigt sich, dass eine aufgeschlossene Haltung gegenüber dem Einsatz in Kindertageseinrichtungen sowie Basiskompetenzen noch keine Umsetzung in der Praxis garantieren. Als weitere Bedingung wurde in den Studien die positive Grundhaltung von Leitungskräften identifiziert. Trotz negativer Stimmen seitens der Gesellschaft, wirkt sich diese positiv sowohl auf die Fortbildungsbereitschaft der Erzieher und Erzieherinnen zu medienpädagogischen Themen aus, als auch auf den Medieneinsatz 
im Alltag. Betont wird zudem, dass die Medienbildung als Querschnittthema zu anderen Bildungsbereichen verstanden werden sollte. Digitale Medien können demzufolge eingesetzt werden, um die Bildungsarbeit in anderen Bereichen zu unterstützen und dabei gleichzeitig Medienkompetenzen aufbauen. Es wurde zudem deutlich, dass auch ohne den Einsatz von technischen Geräten ein Verständnis von Medien, ihren Chancen und Risiken sowie ein kritischer Umgang bei den Kindern geschaffen werden kann. Solche Kenntnisse werden derzeit nur vereinzelt oder lückenhaft im Rahmen der Ausbildung an zukünftige Erzieher und Erzieherinnen vermittelt. In Ermangelung angemessener Zeitressourcen werden zudem erlernte medienpädagogische Inhalte nicht praxisnah eingeübt. Obwohl dies widersprüchlich erscheint zeigt sich vielfach, dass berufserfahrenere Fachkräfte häufiger medienpädagogische Inhalte in ihrer Arbeit aufgreifen als ihre jüngeren Kollegen und Kolleginnen.

Die Studien, die sich mit der Ausbildungssituation beschäftigen, fordern eine stärkere Verankerung medienpädagogischer Inhalte in die Ausbildungscurricula. Neben der Ausbildung gilt die Fort- und Weiterbildung der pädagogischen Fachkräfte als ein wichtiger Baustein der Kompetenzförderung. Obwohl Erzieher und Erzieherinnen häufig Fortbildungen besuchen, werden medienpädagogische Fortbildungsthemen nur selten wahrgenommen. Eine wesentliche Erkenntnis der Studien diesbezüglich ist das Phänomen der „Heilung der Gesunden“, da diese besonders von medienaffinen und aufgeschlossenen Fachkräften genutzt werden. Auch hier kann eine stärkere Verankerung in der Ausbildung zu einer Sensibilisierung der Fachkräfte beitragen.

Inhaltlich liefern die vorliegenden Studien wichtige Ansatzpunkte darüber, wie sich das Feld der Kindertageseinrichtungen bezüglich der digitalen Entwicklungen verhält. Eine wesentliche Forschungslücke ergibt sich aus einer umfassenden, im Idealfall multivariaten Analyse des komplexen Bedingungsgefüges ohne regionale Einschränkungen. So wurden in den Studien Einflussfaktoren für eine rezeptionsorientierte Medienarbeit identifiziert. Dazu zählen z.B. die Haltung der Fachkräfte, ihre Berufserfahrung, ihre selbst eingeschätzte Medienkompetenz, das Vorwissen durch die Ausbildung und der Besuch von Fortbildungen sowie die Unterstützung durch Leitungskräfte und Träger. Hinderlich erweist sich zudem die geringere Bedeutung von Medienpädagogik im Bereich der frühen Bildung im Vergleich zu anderen Bildungsbereichen sowie die meist unzureichenden Bedingungen für die Arbeit mit digitalen Geräten in den Kindertageseirichtungen. Gänzlich fehlt in diesem Bereich bislang die Evaluation bestehender Kompetenzmodelle, wie bspw. das Modell nach Nolte (2014) und dem TPACK-Kompetenzmodell von Koehler und Mishra (2006, siehe auch Bergner et al., 2018), das besonders stark in der schulischen Bildung, Lehrerbildung und Erwachsenenbildung eingesetzt wird. 


\section{Literatur}

* durch Reviewprozess ausgewählte Studie

Bergner, N., Hubwieser, P., Köster, H., Magenheim, J., Müller, K., Romeike, R., Schroeder, U. \& Schulte, C. (2018). Frühe informatische Bildung - Ziele und Gelingensbedingungen für den Elementar- und Primarbereich. In N. Bergner, P. Hubwieser, H. Köster, J. Magenheim, K. Müller, R. Romeike, U. Schroeder \& C. Schulte (Hrsg.), Frühe informatische Bildung - Ziele und Gelingensbedingungen für den Elementar- und Primarbereich. Wissenschaftliche Untersuchungen zur Arbeit der Stiftung „Haus der kleinen Forscher" (Band 9). Opladen, Berlin: Barbara Budrich. https://doi.org/10.3224/8474 2107

BIBB. (2012). Internationale Standardklassifikation im Bildungswesen. BIBB BWP 4/2012, S. 18.

Bilger, F., Behringer, F., Kuper, H. \& Schrader, J. (Hrsg.). (2017). Weiterbildungsverhalten in Deutschland 2016. Ergebnisse des Adult Education Survey (AES). Bielefeld: wbv.

Bostelmann, A. (2018). Die Verantwortung des Kindergartens für die Zukunft. In S. Ladel, J. Knopf \& A. Weinberger (Hrsg.), Digitalisierung und Bildung (S. 179-189). Wiesbaden: Springer. https://doi.org/10.1007/978-3-658-18333-2_10

Büsch, A. \& Demmler, K. (2017). Keine Bildung ohne Medien! (KBoM)-Positionspapier. Medienpädagogik in die frühkindliche Bildung integrieren. Medienimpulse, 55(4), 1-4. https://doi.org/10.21240/mpaed/30/2018.03.01.X

Buschle, C. \& König, A. (2018). E-Learning und Blended-Learning-Angebote. Möglichkeiten beruflicher Weiterbildung für Kita-Fachkräfte. MedienPädagogik, 30, 50-72.

Eder, S., Brüggemann, M. \& Kratzsch, J. (2017). Kinder im Mittelpunkt: Frühe Bildung und Medien gehören zusammen. Positionspapier der GMK-Fachgruppe Kita.

Eder, S., Lehmann, A., Lenich, A., Roboom, S., Seiler, G. \& Wentzel, J. (2013). Medienkompetenz-Kitas NRW. Ein Modellprojekt der Landesanstalt für Medien NordrheinWestfalen. Düsseldorf: LfM.

Feierabend, S., Plankenhorn, T. \& Rathgeb, T. (2015). miniKIM 2014. Kleinkinder und Medien. Basisuntersuchung zum Medienumgang 2- bis 5-Jähriger in Deutschland. Stuttgart: Medienpädagogischer Forschungsverbund Südwest.

${ }^{\star}$ Friedrichs-Liesenkötter, H. (2016). Medienerziehung in Kindertagesstätten: Habitusformationen angehender ErzieherInnen. Wiesbaden: Springer Fachmedien Wiesbaden. https://doi.org/10.1007/978-3-658-12307-9

*Friedrichs-Liesenkötter, H. (2019): ,Wo Medienbildung draufsteht, steckt nicht unbedingt Medienbildung drin'. Medienimpulse, 57(1), 1-47.

Fröhlich-Gildhoff, K., Nentwig-Gesemann, I. \& Pietsch, S. (2011). Kompetenzorientierung in der Qualifizierung frühpädagogischer Fachkräfte. Eine Expertise der Weiterbildungsinitiative Frühpädagogische Fachkräfte (WiFF). München.

Fthenakis, W. E. \& Walbiner, W. (2018). Bildung braucht digitale Kompetenz. 1. Der Einsatz neuer Technologien in der frühen Bildung. Herausforderungen und Perspektiven. Darmstadt: Didacta Verband e.V.

${ }^{*}$ Gessler, A. \& Gruber, V. (2018). Einflussfaktoren auf die Weiterbildungsbeteiligung frühpädagogischer Fachkräfte. Eine empirische Analyse. Der pädagogische Blick, 26(3), $150-162$.

${ }^{*}$ Goetz, I. (2018). Steuerungsmechanismen zur Sicherstellung der Medienbildung in Kindertagesstätten. Dortmund: Universitätsbibliothek Dortmund. 
Graube, G., Jeretin-Kopf, M., Kosack, W., Mammes, I., Renn, O. \& Wiesmüller, C. (2015). Wissenschaftliche Untersuchungen zur Arbeit der Stiftung "Haus der kleinen Forscher" (Band 7). Schaffhausen: SCHUBI Lernmedien AG.

KMK. (2012). Länderübergreifender Lehrplan Erzieherin/Erzieher. Entwurf Stand 01.07.2012.

${ }^{\star}$ Knauf, H. (2019). Digitalisierung in Kindertageseinrichtungen. Ergebnisse einer Fragebogenerhebung zum aktuellen Stand der Nutzung digitaler Medien. Bielefeld working paper, 3, 1-23.

Kunter, M. \& Baumert, J. (2011). Professionelle Kompetenz von Lehrkräften. Ergebnisse des Forschungsprogramms COACTIV. Münster: Waxmann. DOI: 10.1007/978-3-658-009083_13

${ }^{*}$ Marci-Boehncke, G. \& Rath, M. (2013). Kinder - Medien - Bildung. Eine Studie zu Medienkompetenz und vernetzter Educational Governance in der Frühen Bildung. München: Kopäd.

${ }^{\star}$ Meister, D. M., Friedrichs, H., Keller, K., Pielsticker, A. \& Temps, T. T. (2012). Chancen und Potenziale digitaler Medien zur Umsetzung des Bildungsauftrags in Kindertageseinrichtungen in NRW. Paderborn: Universität Paderborn; GMK.

Neuß, N. \& Wiechmann, L. (2017). Medien und Medienpädagogik. In: Hilde von Balluseck (Hrsg.), Professionalisierung der Frühpädagogik. Perspektiven, Entwicklungen, Herausforderungen (2., aktualisierte und überarbeitete Auflage). Opladen, Berlin, Toronto: Barbara Budrich. https://doi.org/10.2307/j.ctvddzfi2.18

*Nolte, D. (2014). Eine Frage der Medienkompetenz? Bedingungen medienpädagogischer Praxis in der Kindertageseinrichtung. Frühe Bildung, 3(4), 214-221. https://doi.org/ $10.1026 / 2191-9186 / \mathrm{a} 000176$

Reichert-Garschhammer, E. (2019). Digitale Transformation im Bildungssystem Kita. In J. Heider-Lang \& A. Merkert (Hrsg.), Digitale Transformation in der Bildungslandschaft den analogen Stecker ziehen? (S. 26-51). Augsburg: Rainer Hampp Verlag. https://doi. org/10.5771/9783957103406-25

Sälzer, H. (2017). Digitale Medienbildung in der frühen Kindheit. Mediennutzungsverhalten in Berliner Kindertagesstätten. Medien + Erziehung, 61(3), 72-77.

${ }^{\star}$ Schubert, G., Eggert, S., Lohr, A., Oberlinner, A., Jochim, V. \& Brüggen, N. (2018). Digitale Medien in Kindertageseinrichtungen. Medienerzieherisches Handeln und Erziehungspartnerschaft. Perspektiven des pädagogischen Personals. Zweiter Bericht der Teilstudie "Mobile Medien und Internet im Kindesalter - Fokus Kindertageseinrichtungen" im Rahmen von MoFam - Mobile Medien in der Familie. München: JFF.

${ }^{*}$ Six, U. \& Gimmler, R. (2007). Die Förderung von Medienkompetenz im Kindergarten. Eine empirische Studie zu Bedingungen und Handlungsformen der Medienerziehung. Berlin: Vistas.

Theunert, H. (2007). Medienkinder von Geburt an. Medienaneignung in den ersten sechs Lebensjahren. München: kopaed. 
Bettina Waffner

\title{
Unterrichtspraktiken, Erfahrungen und Einstellungen von Lehrpersonen zu digitalen Medien in der Schule
}

\begin{abstract}
Die Integration digitaler Medien in die schulische Unterrichtspraxis gewinnt unter Bedingungen des digitalen Wandels zunehmend an Bedeutung. In diesem Zusammenhang bietet das Critical Review der internationalen Forschungsliteratur ein vertieftes Verständnis über Digitalisierungsprozesse in der Schule aus Sicht der Lehrpersonen und die Implikationen für Fort- und Weiterbildungen. Die Synthese der Forschungsergebnisse aggregiert das aktuell verfügbare, evidenzbasierte Wissen über Unterrichtspraktiken, Erfahrungen und Einstellungen von Lehrpersonen zu digitalen Medien in der Schule.

Der metaanalytische Ansatz basiert auf 125 systematisch ermittelten Studien, die in der Zeit von Januar 2010 bis Mai 2019 publiziert worden sind. Im Ergebnis können fünf Synthesis Statements identifiziert werden: (1) Digitale Medien sind bereits im beruflichen Alltag als Kommunikations- und Präsentationsmedien etabliert, wohingegen (2) digitalgestützte pädagogisch-didaktische Unterrichtsformate selten eingesetzt werden. Als Gelingensbedingungen für die Medienintegration werden, (3) neben personalen Faktoren wie extrovertierte, intrinsisch motivierte Charaktereigenschaften der Lehrperson, zeitliche Ressourcen, eine geeignete technische Ausstattung und technischer sowie administrativer Support identifiziert. Lehrpersonen benötigen darüber hinaus (4) neben methodisch-technischen auch pädagogisch-didaktische Kompetenzen für einen Medieneinsatz in der Unterrichtspraxis sowie Kompetenzen, um die erweiterten Möglichkeiten der Kommunikation und Zusammenarbeit nutzen zu können. Als zielführend werden in der aktuellen Forschungsdebatte (5) situierte, digitalgestützte und langfristig angelegte Fortund Weiterbildungen zu medientechnischen und pädagogischen Grundlagen diskutiert, die von Reflexions- und Coachingphasen sowie durch einen Peer-Austausch begleitet werden. Abschließend können auf der Basis des Reviews Forschungsperspektiven identifiziert werden, die bislang unterbelichtet blieben.
\end{abstract}

Schlüsselwörter: Schule, Digitalisierung, Kompetenzen, Weiterbildung, Einstellung, Lehrperson, Critical Review

\section{Teaching practices, experiences and attitudes of teachers towards digital media at school}

The integration of digital media into teaching practices at school is becoming increasingly important under conditions of digital change. In this context, the critical review of the international research literature offers a deeper understanding of the digitisation process from an in-service teachers' perspective. Furthermore, a scientific discussion of implications for teacher professional development is outlined. The synthesis of the research results integrates the currently available, evidence-based knowledge about teaching practices, experiences, and attitudes of teachers towards digital media at school. 
The meta-analytic approach is based on 125 systematically determined studies published between January 2010 and May 2019. As a result, five synthesis statements can be identified: (1) digital media are already integrated in everyday professional life supporting communication and presentation, whereas (2) technology-enhanced teaching is rarely practiced. (3) Significant predictors of teachers' successful use of technology are explored. The findings show that personality factors such as extroverted, intrinsically motivated teacher character traits, time resources as well as technical infrastructure and techni$\mathrm{cal}$ and administrative support are needed as predictors for integrating digital media into teaching practices. In addition to technological competences, teachers also need (4) instructional strategies and methods for media use in teaching practices as well as skills enabling them to use the expanded possibilities of communication and cooperation associated with digital media. The research debate considers (5) technology-enhanced, long-term and teacher professional development focusing on technological and pedagogical principles, which are accompanied by reflection and coaching phases as well as by peer exchange, to promote technology-enhanced innovative teaching practices.

Finally, on the basis of this review, research perspectives can be identified that have so far remained underexposed.

Keywords: school, digitisation, competences, continuing education, attitude, teacher, critical review

\section{Schule in der digital geprägten Welt - Handlungsrahmen von Lehrpersonen}

Vor noch nicht langer Zeit beschäftigten sich nur einige wenige Lehrpersonen an Schulen damit, wie und mit welchem Gewinn digitale Medien in die Unterrichtspraxis integriert werden können. Heute wird diese Frage zunehmend als ein strategisches Thema von Schulentwicklung verstanden, das neben der Unterrichtspraxis von Lehrerinnen und Lehrern ebenso Schulleitungen, kommunale Schulträger und die Landespolitik mit ihren Kultusministerien berührt. In dieser Reichweite stellt das Thema für die Bildungspolitik, für Schulen als Institutionen sowie für Lehrpersonen eine große Herausforderung dar.

Bildungspolitische Reformen müssen auf Veränderung von Unterrichtspraktiken abzielen, die der transformativen Kraft einer pädagogisch-didaktischen Nutzung digitaler Medien gerecht werden. Das berührt auch pädagogische Grundhaltungen von Lehrpersonen, neue Lehr-Lernmedien und Lehrstrategien oder ganz neue Artefakte zum Lernen. Ebenso geht es um Veränderungen der Unterrichtsplanung und um Möglichkeiten der Förderung neuer sozialer Strukturen wie Peer-Interaktionen oder Entscheidungsfindungsprozesse sowohl unter Lehrpersonen als auch in der Unterrichtspraxis unter Schülerinnen und Schülern. Damit wird die Komplexität angedeutet, in der Akteurinnen und Akteure unterschiedlicher Ebenen in den Transformationsprozess involviert sind, um Schule in einer digital geprägten Welt zukunftsfähig zu gestalten. 
Die Implementation und Institutionalisierung digitalgestützter Innovation im Bildungsbereich ist eine "practice to change practices“ (Fullan \& Stiegelbauer, 1991, S. 9), die weit über die Bereitstellung von Technik und einer geeigneten Infrastruktur sowie Lehrerfortbildungen hinaus geht (Kerres \& Waffner, 2019, S. 227). In diesem Verständnis kann Digitalisierung ein Katalysator für Veränderungsprozesse sein (Sipilä, 2014, S. 8), der alle Bereiche der Schulentwicklung von der Unterrichtsentwicklung über die Personalentwicklung und Organisationsentwicklung (Rolff, 2016, S. 20) mit weitreichenden Auswirkungen berührt.

Dabei stehen Schulen in einem Spannungsfeld zwischen dem Anspruch, sich als lernende Organisation (BMBF \& KMK, 2019, S. 3) zu verstehen und gleichwohl Stabilität zu bieten. Nur so können sie ihren gesellschaftlichen Aufgaben gerecht werden, professionelle Bildungsangebote durch kompetentes Lehrpersonal anzubieten und ein verlässliches Lernfeld zu schaffen, das Schülerinnen und Schülern Struktur bietet (Oelkers, 2016, S. 15). Bei dieser herausfordernden Aufgabe arbeiten Schulen unter strukturellen, inhaltlichen und politischen Rahmenbedingungen, in denen die Zuständigkeiten auf verschiedene Akteure verteilt sind. In den Verantwortungsbereich der Kultusministerien fällt die Schulaufsicht, die Ziele und Inhalte des Unterrichts sowie die Struktur des Schulsystems festlegt. Kosten für das Lehrpersonal werden ebenfalls von den Bundesländern übernommen, die auch für deren Fort- und Weiterbildung zuständig sind. Kommunen übernehmen die Schulträgerschaft staatlicher Schulen und Sachkosten für Gebäude und deren Ausstattung. Dazu zählt auch die technische Infrastruktur. Darüber hinaus sind Schulträger für eine langfristige Schulentwicklungsplanung zuständig. Aktuell haben Bund und Länder mit dem DigitalPakt nach einer Grundgesetzänderung, die durch das deutsche föderale Bildungssystem notwendig war, und der Errichtung eines Digitalinfrastrukturfonds die Möglichkeit geschaffen, finanzielle Bundesmittel für die Ausstattung von Schulen mit digitaler Technik zur Verfügung zu stellen. So sinnvoll und notwendig finanzielle Ressourcen für eine zeitgemäße Ausstattung von Schulen sind, so wenig zielführend können innovative Prozesse der Schulentwicklung auf diese Weise erfolgen, denn jede Schule ist durch soziale Unterschiede in der Schülerschaft, durch die Heterogenität des jeweiligen Lehrerkollegiums sowie die baulichen und technischen Gegebenheiten einzigartig trotz der Einbindung in einen gemeinsamen formalen und politischen Rahmen. Daher müssen Schulen diesen Prozess innerhalb des Rahmens selbstständig und selbstverantwortlich gestalten (Rolff, 2011).

Aus diesem Grunde wurden in den vergangenen Jahren politische Reformen umgesetzt, um Schulen mehr Autonomie für Entwicklungsräume zu ermöglichen. Diese können sie nutzen, um den notwendigen Schulentwicklungsprozess individuell zu gestalten. Wie der Bildungsauftrag in einer digitalisierten und mediatisierten Gesellschaft erfüllt werden kann, ist eine komplexe Herausforderung. Normative Konzepte, wie z.B. der Bildungsanspruch, jedem Menschen Teilhabe an den kulturellen Errungenschaften der Gesellschaft zu ermöglichen, müssen mit den empirisch 
erfassten Wirkungen bildungspolitischer Instrumente und Implementations- sowie Institutionalisierungswegen in der Bildungspraxis konfrontiert werden.

Hattie (2009) hat bereits vor über 10 Jahren in seiner vielbeachteten Meta-Studie empirisch nachgewiesen, dass Lehrpersonen die wichtigsten Garanten eines erfolgreichen und wirksamen Unterrichts seien. Der vorliegende Text stellt als Critical Review die wichtigsten empirischen Forschungsergebnisse und theoretisch-konzeptionellen Überlegungen hinsichtlich der Bedeutung der Digitalisierung für das pädagogische Personal in einer Synthese dar.

Wie ist die persönliche Haltung oder Einstellung zur Digitalisierung in der pädagogischen Arbeit und was bedeutet diese für die Nutzung digitaler Medien? Welche Kompetenzen benötigen Lehrpersonen in einer digital geprägten Welt? Wie sind Fort- und Weiterbildungen im Konnex der Digitalisierung gestaltet und welche Entwicklungen können schließlich im schulischen Bereich im Unterricht und der pädagogischen Arbeit beobachtet werden?

Abschließend werden Forschungsdesiderata identifiziert und die Implikationen der Analyse für die Schulpraxis sowie mögliche methodische und politische Implikationen diskutiert.

\section{Recherche und systematische Auswahl der Studien}

Eine ausführliche Darstellung des Vorgehens bei der Recherche findet sich zu Beginn dieses Bandes in einem eigenen Beitrag. Im Folgenden werden die Spezifika des Bildungssektors Schule bei der Recherche sowie das systematische Vorgehen bei der Auswahl der Titel dargestellt, die in das Critical Review eingingen.

\subsection{Sektorenspezifische Suchbegriffe der Recherche}

Die Suchbegriffe für die Recherche orientieren sich (1) an der Frage der Bedeutung der Digitalisierung im Bildungssektor Schule, der hier allgemeinbildende Schulen umfasst. Das Thema des Critical Reviews lässt sich (2) in die Teilthemen persönliche Haltung und Einstellungen sowie notwendige Kompetenzen von Lehrpersonen und Fort- und Weiterbildung untergliedern und (3) umfasst die Recherche sowohl deutschsprachige als auch englischsprachige Literatur. Insofern werden Suchbegriffe sowohl in deutscher als auch in englischer Sprache verwendet.

Bei der Beschäftigung mit dem Thema digitale Medien in der Schule kann sowohl in der Bildungsforschung als auch in der Bildungspraxis festgestellt werden, dass Begriffe nicht einheitlich verwendet werden, so dass in die Recherche verschiedene Suchbegriffe einflossen wie neue Technologien, neue Medien, computergestützter Unterricht, Multimedia und Tablet-PC. Im englischsprachigen Bereich werden häufig 
Begriffe wie information and communication technology (ICT), educational technology, blended learning, e-learning oder social media verwendet.

Das pädagogische Personal in der Schule wird in der Forschung meist als Lehrer oder Lehrerin bezeichnet. Häufig wird auch die Partizipkonstruktion oder der Begriff Lehrkraft genutzt. In der englischen Form sind teacher die Personengruppe, die hier betrachtet werden. Teilweise wird die Schulform, wie z. B. secondary school teachers hinzugefügt.

\subsection{Auswahl und Kategorisierung der Studien in drei Analysesträngen}

Die Recherche in den Datenbanken für den Suchzeitraum zwischen Januar 2010 und Mai 2019 ergibt eine Trefferanzahl von 3380 Titeln. Ziel der systematischen Auswahl, der in das Critical Review eingehenden Titel, ist es, diejenigen zu identifizieren, die die aktuelle internationale und deutsche Forschungsdebatte möglichst vollständig erfassen. Dadurch wird eine systematische Forschungssynthese ermöglicht, die drei Ziele verfolgt. Zum einen (1) wird die bildungswissenschaftliche Debatte über die Bedeutung der Digitalisierung für das Lehrpersonal an allgemeinbildenden Schulen erfasst. Das ermöglicht (2) Forschungsdesiderata zu erkennen, um vertiefte Analysen daran anschließen zu können. Zum anderen kann (3) die Forschungssynthese auch eine Grundlage für Forschungsstrategien im Feld (Tippelt \& Schmidt-Hertha, 2018, S. 1424) oder für bildungspolitische und bildungspraktische Entscheidungen bieten.

Um das Literaturkorpus systematisch und begründet zu reduzieren, wird in einem ersten Schritt auf die Wege der Wissenschaftskommunikation rekurriert und der Ort in den Blick genommen, an dem neue wissenschaftliche Erkenntnisse publiziert werden. Dieses geschieht im Wesentlichen auf wissenschaftlichen Tagungen und in Fachzeitschriften. In Monographien und Sammelwerken erfolgt häufig eine Vertiefung der bereits veröffentlichten Forschungsergebnisse. So kann davon ausgegangen werden, dass der wissenschaftliche Diskurs durch Zeitschriftenartikel nahezu vollständig erfasst werden kann. Das Korpus wird durch die Konzentration auf den Dokumententyp Zeitschriftenartikel auf 2078 Titel reduziert.

Im weiteren Vorgehen werden aus dem Korpus drei verschiedene Analysestränge identifiziert, die zunächst getrennt voneinander betrachtet werden. Das scheint deshalb sinnvoll zu sein, da die deutsche Debatte um diese Frage Spezifika aufweisen kann, die im Bildungssystem wie dem Föderalismus und der bildungspraktischen Tradition begründet sein können. Gleichzeitig finden sich im internationalen Kontext nur wenige wissenschaftliche Beiträge aus dem deutschen Sprachraum. Die separate Betrachtung der ausschließlich deutschsprachigen Beiträge ermöglicht, Gemeinsamkeiten und Unterschiede zur internationalen Debatte zu identifizieren.

Der Analysestrang 1 umfasst die Forschungsliteratur, die am meisten Beachtung durch Zitationen findet. Da zwischen dem Erscheinen einer Publikation und einer messbaren Anzahl von Zitationen ein Zeitverzug liegt, werden für diesen 
Analysestrang Titel ausgewählt, die bis 2017 erschienen sind. Damit liegen zwischen dem Erscheinen und der Ermittlung der Zitationshäufigkeit mindestens eineinhalb Jahre.

Die Zitationen werden bei Google Scholar ermittelt, wohlwissend, dass diese weder zwingend durch wissenschaftliche Publikationen erfolgen noch durch ein wissenschaftlich kuratiertes System wie beispielsweise bei Web of Science oder Scopus ermittelt werden. Allerdings hat das automatische System von Google Scholar erhebliche Vorteile wie Martín-Martín, Orduna-Malea, Thelwall und López-Cózar kürzlich nachgewiesen haben:

„[...] this study gives the first systematic evidence to confirm [...] that citation data in GS has reached a high level of comprehensiveness [...]. It surpasses WoS and Scopus numerically in all areas of research and is greatly superior in the areas where WoS and Scopus have a poor coverage, including the Social Sciences and Humanities“ (Martín-Martín et al., 2018, S. 21).

Bei Google Scholar sind 1566 Titel von den 1803 Titeln auffindbar. Davon werden 103 Titel 15-mal und häufiger pro Jahr zitiert. Die Volltexte dieser Titel werden mittels eines Schemas kodiert, das eine systematische Bewertung der Artikel hinsichtlich formaler und inhaltlicher Kriterien sowie qualitativ hinsichtlich wissenschaftlicher Gütekriterien ermöglicht. Es besteht aus drei Teilen: (1) Beschreibung (Publikationstyp, Weg der Literaturbeschaffung, Art der Studie, Forschungsdesign, Geographischer Fokus, Passung zum Kontext des Dossiers), (2) Inhalt (Ziele der Studie, Fragestellung und/oder Hypothese, Ergebnisse der Studie, Passung zur Fragestellung des Dossiers), (3) Wissenschaftliche Bewertung (Validität, Reliabilität/ Plausibilität, Generalisierbarkeit, Zusammenfassende Bewertung). In jedem Teil des Kodierschemas gibt es Kriterien, die einen Titel aus dem Literaturkorpus ausschließen lassen. Wenn eine Studie nicht in den Kontext des Critical Reviews passt, wenn sie z.B. explizit auf einen anderen nationalen Kontext oder einen anderen Bildungssektor abzielt, dann wird sie ausgeschlossen. Das gleiche gilt für Studien, deren Forschungsfragen keine Anknüpfungspunkte an die hier zugrundeliegende Fragestellung aufweisen. Ein drittes Kriterium rekurriert auf die wissenschaftlichen Gütekriterien Validität, Reliabilität und Generalisierbarkeit, die auf einer Dreierskala bewertet und zusammenfassend ein Ein- oder Ausschlusskriterium darstellen. ${ }^{1}$

Von den 103 Titeln, die mindestens 15 Mal pro Jahr zitiert wurden, werden 69 Titel nach der Kodierung ausgeschlossen, so dass 35 Titel die Basis der Analyse und Synthese des Analysestrangs 1 bilden. Wie zu erwarten, sind die im internationalen Kontext meist beachteten Titel alle englischsprachig.

Um die Forschungsdebatte im deutschen Kontext $\mathrm{zu}$ ermitteln, werden im Analysestrang 2 alle 319 deutschsprachigen Titel des Korpus einer ersten Durchsicht der Titel und Abstracts hinsichtlich ihrer inhaltlichen Relevanz für die Fragestellung

1 Eine genauere Darstellung des Kodierschemas findet sich in der Einleitung dieses Bandes. 
des Critical Reviews und des wissenschaftlichen Anspruches unterzogen. In dieser ersten Durchsicht werden bereits 244 Titel ausgeschlossen. Die Volltexte von 75 Titel werden mittels des Kodierschemas bewertet. 16 Titel finden Eingang in den Analysestrang 2.

Der Analysestrang 3 ermittelt die Ergebnisse der Literatur von 2018 bis Mai 2019. Hier ist interessant, inwiefern aktuelle Entwicklungen zu beobachten sind. Alle 275 Titel aus der Recherche werden in diesem Analysestrang vollständig erfasst und anhand der Titel und Abstracts ähnlich geprüft wie diejenigen im Analysestrang 2. Es ist $\mathrm{zu}$ beachten, dass sich unter den 275 Titeln 21 deutschsprachige befinden, die auch bereits in den Analysestrang 2 eingingen. Soweit diese Titel inhaltlich in die Analyse und Synthese des Analysestrangs eingegangen sind, wurde dieses explizit gemacht. Ein überraschend hoher Anteil konnte mit 176 Titeln in die Volltextprüfung und Kodierung übernommen werden. Insgesamt gingen 74 Titel in den Analysestrang 3 ein.

Durch diese systematische Vorgehensweise, die den Anspruch erhebt, die aktuelle, breit beachtete Forschung $\mathrm{zu}$ dem Thema $\mathrm{zu}$ erfassen, konnten insgesamt 125 Titel der recherchierten 3380 Titel ermittelt werden, die in die folgende Forschungssynthese eingehen.

\section{Analyse aktueller internationaler und deutscher Forschung}

Im Folgenden wird eine Beschreibung der Studien sowie eine Synthese der Forschungsergebnisse erfolgen. Dieses Vorgehen ermöglicht „[...] meaning, structure, and order to data [...]" (Anfara, Brown \& Mangione, 2002, S. 31) vorzunehmen, um Kategorien und Muster zu erkennen, die weiterführende Erkenntnisse ermöglichen.

Es werden die 125 Studien in den Blick genommen, die im internationalen und im deutschen Raum zum einen viel beachtet sind, zum anderen eine hohe Relevanz für die Fragestellung dieses Critical Reviews haben und die von hoher wissenschaftlicher Qualität sind. Ziel ist es, die Agenda zu identifizieren, die den Forschungsdiskurs prägt. $\mathrm{Zu}$ diesem Zweck werden nach einer Beschreibung des Forschungsdesigns der Studien, die Forschungsthemen in den Blick genommen und in Kategorien verdichtet. Das ermöglicht, Rückschlüsse auf die Forschungsagenda zu ziehen. In einem nächsten Schritt werden die relevanten Ergebnisse verdichtet dargestellt. In der Forschungssynthese werden ausschließlich die Forschungsergebnisse berücksichtigt, deren Fokus explizit auf der Bedeutung der Digitalisierung für Lehrpersonen liegt. $^{2}$

2 Aus Gründen der Transparenz soll Erwähnung finden, dass die Studien Klemm (2018), Palkowitsch-Kühl (2018) und Swertz (2018) sowohl in dem Analysestrang 2 als auch 3 berücksichtigt werden. 


\subsection{Forschungsdesign der Studien}

Die Studien werden hinsichtlich des Entstehungsortes, des Forschungsdesigns und der Art der Datenerhebung sowie der Samplegröße (n) näher betrachtet. Eine detaillierte tabellarische Beschreibung der Studien befindet sich im Anhang. ${ }^{3}$

Über $90 \%$ der Studien des Analysestrangs 1 sind empirische Untersuchungen, die das gesamte Spektrum der Forschungsdesigns von quantitativen, qualitativen und Mixed Method Studien umfassen. Die überwiegende Mehrzahl dieser Studien stammt aus den USA oder Kanada (14 Studien), während asiatische/türkische Studien mit sieben Studien ebenfalls stark vertreten sind. Hier fällt auf, dass Letztere ausschließlich quantitativ angelegt sind. Die einzige aus Deutschland stammende Studie legen Drossel, Eickelmann und Gerick (2017) vor. Sie ermitteln länderübergreifend begünstigende Faktoren für Mediennutzung im Klassenraum auf der Basis von ICILS-Personendaten (Bos et al., 2014).

Bei den deutschsprachigen Studien des Analysestrangs 2 ist auffallend, dass ein Viertel der Studien theoretisch-konzeptionell angelegt sind, was einen erheblich höheren Anteil ausmacht als in den Studien, die im internationalen Kontext am meisten Beachtung fanden.

Bei den Studien von 2018 bis Mai 2019 des Analysestrangs 3 kann festgestellt werden, dass es im Vergleich zu der Literatur, die am häufigsten zitiert wurde, einen erheblich höheren Anteil asiatischer/türkischer Studien gibt und auch afrikanische Studien für das Thema relevant sind. Unter den asiatischen Studien befinden sich anders als im Analysestrang 1 auch indische Studien. Es liegt die Vermutung nahe, dass auch in den Jahren 2010 bis 2017 Studien in diesen Regionen entstanden, diese aber international weniger Beachtung fanden, sodass sie hier aus den dargestellten forschungspraktischen Gründen ausgeschlossen wurden. Ein weiterer Befund ist erwähnenswert. Europäische Studien sind im internationalen Vergleich häufiger qualitativ angelegt, was damit korrespondiert, dass deutschsprachige Studien öfter theoretischkonzeptionell angelegt sind.

Insgesamt werden die Daten in einem großen Teil der Studien durch Befragungen erhoben, in denen 20 bis 2500 Personen befragt wurden. Den überwiegenden Anteil der Befragten bilden Lehrpersonen. Auch Interviews werden neben Beobachtungen besonders häufig genutzt. Insbesondere in den neuesten Studien werden die Daten aber auch auf andere Weise erhoben z. B. durch Fokusgruppen, Videos, Posts in sozialen Netzwerken oder durch Unterrichtskonzepte.

Die Datenbasis der Studien ist bezüglich der Samplegröße und der Art der Datenerhebung vielfältig und verweist nicht zwingend auf die Qualität der jeweiligen Studien. Vielmehr zeigt sich die große Bandbreite bei der Anlage der Studien und die Vielzahl von Perspektiven auf den Untersuchungsgegenstand.

3 Tabellen 1 bis 3 des Anhangs 


\subsection{Forschungsthemen der Studien}

Die Forschungsthemen der Studien sind weniger heterogen als die Bandbreite der Forschungsdesigns erwarten lässt und liegt verständlicherweise nahe an den Fragestellungen der Recherche. Gleichwohl erfolgt die Kategorienbildung in einem offenen Kodierverfahren, bei dem Mehrfachzuordnungen möglich sind, da bisweilen in den Studien mehr als ein Untersuchungsgegenstand in den Blick genommen wird. Es lassen sich sieben Kategorien unterscheiden: (1) Kompetenzen und berufliche Entwicklung von Lehrpersonen, (2) Einstellungen und Haltung von Lehrpersonen, (3) Art der Mediennutzung, (4) Wirkung mobiler Mediennutzung in der Wahrnehmung der Lehrpersonen, (5) Theorie-Praxis-Beziehung, (6) Gelingensbedingungen und Hindernisse für Mediennutzung in der Unterrichtspraxis und (7) Korrelationen zwischen Mediennutzung und einer weiteren Variable. Im Folgenden werden diejenigen Forschungsthemen skizziert, die besonders häufig vertreten sind. ${ }^{4}$

Ein signifikant hoher Anteil der meistzitierten Studien aus den Jahren 2010 bis 2017 (Analysestrang 1) untersucht Korrelationen. Dabei kann keine geographische Präferenz für diese Art der Untersuchung festgestellt werden. Allerdings liegt ein inhaltlicher Schwerpunkt auf einer Untersuchung der Korrelation zwischen Mediennutzung in der Unterrichtspraxis als abhängige Variable und verschiedenen unabhängigen Variablen wie der Einstellung, den personalen Eigenschaften und Kompetenzen von Lehrpersonen, die Besonderheit eines Schulfaches sowie Rahmenbedingungen der einzelnen Schule wie die technische Infrastruktur. In einigen Studien ist die Mediennutzung die unabhängige Variable und es wird untersucht, ob beispielsweise eine häufigere Nutzung von Medien zu einer Einstellungsänderung bei Lehrpersonen führt oder ob es Zeitersparnisse gibt. Andere Studien untersuchen den Mehrwert digitaler Medien in Abhängigkeit von individuellen, institutionellen oder systemischen Faktoren.

Neben Korrelationen beschäftigt sich ein Großteil der Studien mit Kompetenzen. Dabei wird deutlich, dass die Frage, was unter Kompetenzen von Lehrpersonen in einer digital geprägten Welt $\mathrm{zu}$ fassen ist, sehr unterschiedlich verstanden wird. Aus diesem Grund werden zwei Kategorien von Kompetenzen unterschieden. (1) Kompetenzen, die sich an dem TPACK-Modell (Mishra \& Köhler, 2006) orientieren (2) Kompetenzen, die auf die sogenannten „21st century skills" (Soffel, 2016) rekurrieren. Neben der Frage, welche Art von Kompetenzen in einer digital geprägten Welt für Lehrpersonen notwendig seien, werden auch Fragen zur Kompetenzentwicklung untersucht. Dabei steht im Vordergrund wie berufliche Fort- und Weiterbildung in der digitalen Welt konzipiert sein sollten, welche neuen Anforderungen an Lehrpersonen in der digital geprägten Welt gestellt werden und wie „TPACKing“ (Olofson, Swallow \& Meredith, 2016) erfolgt. Einige Studien behandeln explizit die Frage, inwieweit digitale Medien, digitale Tools oder soziale Netz-

4 Tabellen 4 bis 6 des Anhangs 
werke in der beruflichen Fort- und Weiterbildung eingesetzt werden. Interessant ist, dass die Hälfte der deutschsprachigen Studien Kompetenzen oder die Kompetenzentwicklung thematisieren.

In allen drei Analysesträngen werden Gelingensbedingungen für Medienintegration in der Unterrichtspraxis untersucht. In den neuesten Studien werden auch Hindernisse auf dem Weg der Medienintegration in der Regel als Fehlen der Gelingensbedingungen identifiziert. Wenn beispielsweise in einigen Studien ${ }^{5}$ eine geeignete technische Infrastruktur als Gelingensbedingung ermittelt wird, so wird in anderen Studien ${ }^{6}$ das Fehlen geeigneter Technik als Hindernis identifiziert. Aus diesem Grund können hier beide Seiten der Medaille gemeinsam betrachtet werden.

Die Einstellung und Haltung von Lehrpersonen zu digitalen Medien ist in allen Analysesträngen ein wichtiger Untersuchungsgegenstand. Einige Studien ${ }^{7}$ richten den Fokus weniger auf digitale Medien als stärker auf die pädagogische Grundhaltung oder die Konstruktion von Begründungen für die Mediennutzung.

In neuesten Studien erfolgt eine Untersuchung der Beziehung zwischen Theorie und Praxis.

Es könnte ein interessanter Hinweis sein, dass der Schwerpunkt der Forschungsthemen der meistbeachteten, internationalen Studien auf begünstigende Faktoren der Medienintegration in die Unterrichtspraxis liegt, während in deutschsprachigen Studien stärker Einstellungen und Kompetenzen von Lehrpersonen thematisiert werden. In diesem Zusammenhang fällt auf, dass Kompetenzentwicklung ausschließlich in Form traditioneller Lehrerfortbildungen gedacht wird.

\subsection{Synthese der Studienbefunde}

\subsubsection{Kompetenzen und berufliche Entwicklung von Lehrpersonen}

Die klassischen grundlegenden Anforderungen an die pädagogische Arbeit in der Schule haben auch in der digital geprägten, vernetzten Welt Bestand. „Schule soll einerseits die optimale Entfaltung der Möglichkeiten und Fähigkeiten des Individuums sowie andererseits die selbstständige Teilhabe und Partizipation an Gesellschaft und Kultur unterstützen und fördern" (Ministerium für Schule und Weiterbildung des Landes Nordrhein-Westfalen, 2014). Gleichwohl ändern sich aber der Zugang zu Informationen und Wege der Kommunikation, wodurch neue Erwartungen an das Lehrpersonal sowohl im Umgang mit digitalen Medien in einer technisch gestützten Unterrichtspraxis gestellt werden als auch in der Zusammenarbeit unter Lehrpersonen. Neben Fachwissen und pädagogisch-fachdidaktischen Kenntnissen sind ebenso auch methodisch-technische Kenntnisse zur Nutzung di-

\footnotetext{
Sauers \& McLeod, 2018; Pima, 2019; Pareja et al., 2018; Khlaif, 2018

Kearney et al., 2018; Degirmenci, 2018; Kilinc et al., 2018

Holmberg et al., 2018; Lynch et al., 2014
} 
gitaler Medien in der Unterrichtspraxis erforderlich. Es ist empirisch nachweisbar, dass zwar Letzteres bei Lehrpersonen weniger stark ausgeprägt ist, ${ }^{8}$ aber insgesamt können dem Lehrpersonal durchaus moderate bis gute technische Kompetenzen bescheinigt werden. ${ }^{9}$ Die Integration digitaler Medien in die Unterrichtspraxis könne allerdings nur dann erfolgreich gelingen, wenn sich der technologische zu einem stärker pädagogischen Fokus verschiebe.

Darüber hinaus brauche es Kompetenzen, um Regeln und Muster neuartiger Kommunikation z.B. in sozialen Netzwerken zu verstehen und zu reflektieren, damit diese gewinnbringend in der Praxis eingesetzt werden können (Klemm, 2018). In dem Zusammenhang wird darauf verwiesen, dass eine veränderte Raumwahrnehmung in der digital geprägten Welt das Konzept einer mündigen, bürgerschaftlichen Teilhabe an der Gesellschaft verändere. Da diese eng verknüpft sei mit den „[...] Geographien des Alltags [...]“" (Schulze et al., 2015, S. 140), die heute digital organisiert seien, brauche es Kompetenzen zur geomedialen Informationsbearbeitung und die Fähigkeit, Geomedien sinnvoll nutzen zu können. ${ }^{10}$

Es können drei Kompetenzkategorien auf Basis aktueller Kompetenzmodelle für Lehrpersonen identifiziert werden, die in einer digital geprägten Welt an Bedeutung gewinnen: (1) humanistic knowledge rekurriert auf Alltagskompetenzen und berufliche Fähigkeiten aber auch auf kulturelle Kompetenzen und auf Werte wie ethische und emotionale Aufmerksamkeit, (2) foundational knowledge umfasst neben Fachkenntnissen auch interdisziplinäre Kenntnisse und digital literacy. Zu (3) meta knowledge zählen Aspekte wie Kreativität und Innovation, Problemlösungskompetenz und die Fähigkeit zu kritischem Denken sowie Kommunikation und Kollaboration. Dieses beinhaltet, sich über alle Medien hinweg - mündlich, schriftlich, nonverbal und digital - klar artikulieren und respektvoll zuhören zu können (Kereluik, Mishra, Fahnoe \& Terry, 2013, S. 130-131). Das Lehrpersonal sei mit erweiterten Möglichkeiten der Kommunikation über Ländergrenzen hinweg und mit weltweiter Vernetzung durch Globalisierungsprozesse konfrontiert. Das erfordere ein hohes Maß an kultureller Kompetenz und emotionaler Aufmerksamkeit sowie Führungsqualitäten, „[...] to facilitate not just interactions, but meaningful interactions and relationships [...]" (Hervorhebungen im Original, ebd., S. 133).

Es stellt sich die Frage, wie die in der digitalen Welt zunehmend wichtiger werdenden Kompetenzen von Lehrpersonen erworben werden. Insbesondere im deutschsprachigen Raum fällt auf, dass selten über klassische Lehrerfortbildungen hinausgedacht wird. Derzeit werden die mehrheitlich hochspezialisierten Fortbildungsprogramme als wenig praxisnah und transferorientiert wahrgenommen. Sie gingen vorbei an den Bedarfen des Lehrpersonals, das eher medientechnische und

8 Mtebe \& Raphael, 2018; Ozdamli \& Uzunboylu, 2015

9 Mesfin, Ghinea, Grønli \& Hwang, 2018; Mtebe \& Raphael, 2018; Muhaimin et al., 2019; Reichert \& Mouza, 2018; Yildiz, 2018

10 Der spatial-citizenship-Ansatz (Gryl \& Jekel, 2012) verweist auf diesen Aspekt und umfasst die bürgerschaftliche Teilhabe, mittels digitaler Medien, gesellschaftliche Diskurse zu räumlichen Konstruktionen zu initiieren und zu hinterfragen (Schulze et al., 2015). 
pädagogische Grundlagen vertiefen will. ${ }^{11}$ In der internationalen Debatte werden in diesem Kontext auch Möglichkeiten eines Einsatzes mobiler digitaler Medien, sozialer Netzwerke und MOOCs diskutiert. ${ }^{12}$

In der neuesten Forschung wird hervorgehoben, dass die Potenziale digitaler Medien für das Lernen in der Schule besonders in lernerzentrierten Unterrichtsszenarien zum Tragen kämen, die mit einer konstruktivistischen pädagogischen Grundhaltung der Lehrperson einhergingen. ${ }^{13}$ Um diese zu fördern, könnten in langfristig angelegten beruflichen Fort- und Weiterbildungen traditionelle, stärker lehrerzentrierte, behavioristische pädagogische Grundhaltungen reflektiert und Lehrpersonen mit konstruktivistisch angelegten Unterrichtsformaten vertraut werden. Das setze aber voraus, dass Fort- und Weiterbildungen Reflexions- und Coachingelemente sowie die Möglichkeiten des Peer-Austausches enthielten. ${ }^{14}$ Sie müssten auf drei zentralen Säulen aufgebaut sein: (1) Es brauche zum einen eine gemeinsame Vision über den Sinn digitalgestützter Lernsettings, zum anderen (2) Netzwerke und Communities für den gegenseitigen Erfahrungs- und Informationsaustausch und zum dritten (3) einen wissenschaftlichen Forschungsansatz, der Innovation ermögliche sowie Praxis und Wissenschaft stärker miteinander verzahne, wie es ein DesignBased-Research-Ansatz leistet (Albion et al., 2015, S. 660).

\subsubsection{Pädagogische Haltung von Lehrpersonen und Einstellungen zu digitalen Medien}

Die Einstellung des Lehrpersonals zu digitalen Medien und die pädagogische Grundhaltung scheinen als „[...] main predictors of teachers' classroom technology use [...]" (Hsu, 2016 zitiert nach Miller et al., 2003, S. 37) gelten zu können. Die meisten befragten Lehrpersonen gaben an, digitalen Medien positiv gegenüber zu stehen und diese häufig in der Unterrichtspraxis und zur Unterrichtsvorbereitung zu nutzen. ${ }^{15}$ Allerdings sei erwähnt, dass nicht ganz klar ist, was sich genau hinter der Formulierung positive Einstellung verbirgt. ${ }^{16}$ Trotz dieser Einschränkung kann festge-

11 Interessant ist der Befund, dass obwohl der Wunsch des Lehrpersonals bestehe, an Lehrerfortbildungen teilzunehmen, $40 \%$ der Befragten in den vergangenen drei Jahren keine Fortbildung besucht haben (Hankmann, 2014, S. 22). Zu dem Thema ebenfalls Knaus, 2012; Ludewig, Knaus \& Döring, 2013; Palkowitsch-Kühl, 2018

12 Abubakar \& Salmanu, 2018; Carpenter \& Krutka, 2014; Carpenter \& Krutka, 2015; Castaño-Muñoz, Kalz, Kreijns \& Punie, 2018; Koukis \& Jimoyiannis, 2019; Rosell-Aguilar, 2018 Yildiz, 2018

13 Daher, Bayàa \& Anabousy, 2018; Frumin, 2018; Koh, 2019; Kul, 2018; Looi, Sun, Kim \& Wen, 2018; Nugent et al., 2018

14 Daher et al., 2018; Frumin et al., 2019; Kali, Levy, Levin-Peled \& Tal, 2018; Kul, 2018; Looi et al., 2018; Mavrotheris, 2019; Meletiou-Mavrotheris, Paparistodemou \& Christou, 2019; Nugent et al., 2018; Tsouccas \& Meletiou-Mavrotheris, 2019

15 Hankmann, 2014; Ludewig et al., 2013

16 Tsouccas und Meletiou-Mavrotheris resümieren, Lehrpersonen hätten „[...] positive attitudes towards the adoption of mobile apps [...]" (2019, S. 15), auch Jong, Grundmeyer und Anderson beziehen sich auf mobile Medien: „[...] teachers feel positive about the uses and the importance of mobile technology in the classroom [...]" $(2018$, S. 12), ebenso wie Howlett und Waemusa „[...] 
stellt werden, dass eher eine wohlwollende als ablehnende Haltung des Lehrpersonals gegenüber digitalen Medien im Unterricht vorzuherrschen scheint. Das beziehe sich im Wesentlichen auf die Nutzung von Computern und Smartboards im Unterricht. Die Skepsis gegenüber eines Einsatzes des Internets ist erheblich größer und steigert sich noch bei der Beurteilung sozialer Netzwerke für das Lernen (Sarac, 2018).

Was aber durchaus verwundert, ist die „[...] Diskrepanz zwischen kommunizierter Haltung und der mangelnden handlungspraktischen Umsetzung [...]" (Brüggemann, 2014, S. 65) eines Medieneinsatzes in der Unterrichtspraxis. Zahlreiche Studien untersuchen, warum eine positive Haltung gegenüber digitalen Medien zwar eine notwendige, jedoch keine hinreichende Bedingung für eine pädagogischdidaktisch sinnvolle Integration in die Unterrichtspraxis darstelle: Erstens (1) fehle Lehrpersonen „[...] the competencies of using mobile devices [...] to pass into m[obile]-learning applications“ (Ozdamli \& Uzunboylu, 2015, S. 169), zweitens (2) zeichnen sich Lehrpersonen häufig „[...] durch medienkritische und bewahrende Haltungen aus, während ein anderer Teil sich vornehmlich an der Vermittlung technisch-instrumenteller Nutzungskompetenzen orientiert. [...]“ (Brüggemann, 2014, S. 71). Bei der Nutzung digitaler Medien würde daher selten eine Orientierung an (medien-)pädagogischen Zielen erfolgen. ${ }^{17}$ Drittens (3) wird auf eine Paradoxie verwiesen: Auf der einen Seite bestehe der normative Anspruch, Schülerinnen und Schülern eine verantwortliche Nutzung digitaler Medien zu vermitteln. Auf der anderen Seite werde ihnen im Unterricht eine eigenverantwortliche Nutzung häufig nicht zugestanden (Brüggemann, 2014). Ein vierter Aspekt (4) bezieht sich auf die fälschliche Annahme, die Nutzung von Medien durch Lehrpersonen trügen per se zu beruflicher Professionalisierung bei. Diese erfolge jedoch nur dann, wenn der Indikator nicht ist, ob, sondern wie Medien eingesetzt werden (Fransson, Holmberg, Lindberg \& Olofsson, 2019).

Die pädagogische Grundhaltung der Lehrperson scheint insbesondere auf die Art der Mediennutzung einen Einfluss zu haben. Lehrpersonen, die einem behavioristischen Paradigma folgen und Lernende durch den Lernstoff hindurchführen, würden insbesondere Präsentationsmedien und sogenannte closed Apps bevorzugen, die gut in die traditionelle vertraute Unterrichtspraxis passen. Open Apps fokussieren auf Lernartefakte von Schülerinnen und Schülern sowie auf Wissenskommunikation, die sich auf eine stärker konstruktivistische pädagogische Haltung zurückführen lassen (Fransson et al., 2019).

Abschließend kann festgehalten werden, dass die Wirkung digitaler Medien in einer sich verändernden Welt für das Lernen in der Schule in seiner Bandbreite von dem Lehrpersonal mehrheitlich nicht erfasst wird, da Medien meist nicht als inte-

all teachers [...] agreed on the benefits and promotion of mobile devices [...]“ (2018, S. 374). Muslem, Yusuf und Juliana rekurrieren allgemeiner auf information and communication technology (ICT) und formulieren, es herrschten „[...] positive perceptions [...]“ (2018, S. 18), während Mwila von „[...] positive attitudes [...]“ $(2018$, S. 223) und Biçak von „[...] positive opinions [...]“ (2019, S. 15) sprechen.

17 Brüggemann, 2014; Lynch \& Redpath, 2014 
graler Bestandteil des Lernprozesses verstanden werden. Vielmehr werden sie eher als ein modernes Tool zur Gestaltung des Unterrichts wahrgenommen (DeCoito \& Richardson, 2018, S. 362).

\subsubsection{Art der Mediennutzung durch Lehrpersonen}

Aktuell wird in der wissenschaftlichen Forschung nicht nur die Quantität in der Unterrichtspraxis empirisch untersucht, sondern auch die Art der Mediennutzung und damit ihre Qualität. Es kann nachgewiesen werden, dass digitale Medien durchaus bereits einen Platz im schulischen Alltag des Lehrpersonals einnehmen. Sie werden mehrheitlich in der Unterstützung der Kommunikation und Präsentation von Inhalten und ebenso als Ersatz für traditionelle Medien wie etwa Arbeitsblätter eingesetzt. ${ }^{18}$ „ICT practices largely tend to be a replica of habitual teaching practices but using digital devices in the place of textbooks" (Rana, Greenwood, Fox-Turnbull \& Wise, 2018, S. 162). Dabei bevorzugen Lehrpersonen eher fest installierte Geräte beispielsweise in extra eingerichteten Computerräumen als mobile Endgeräte in den Klassenzimmern (Pérez-Paredes, Ordoñana Guillamón \& Aguado Jiménez, 2018).

Aber es können durchaus auch andere Arten der Mediennutzung beobachtet werden. So setzen einige Lehrpersonen digitale Medien auch in lernerzentrierten Unterrichtsformaten ein, um „[...] socializing and peer instruction [...]" (Nami, Marandi \& Sotoudehnama, 2018, S. 375) sowie den Wissensaufbau zu fördern (dazu ebenfalls Herro, Quigley \& Jacques, 2018).

\subsubsection{Wirkung mobiler Mediennutzung in der Wahrnehmung des Lehrpersonals}

In der Forschung seit 2018 wird auch thematisiert, inwiefern das Lehrpersonal Wirkungen eines Einsatzes mobiler Endgeräte in der Unterrichtspraxis sowohl bei dem Lehrpersonal als auch bei Schülerinnen und Schülern wahrnimmt. Beim Lehrpersonal kann gezeigt werden, dass die Nutzung mobiler Medien dazu führen kann, dass sich ihre berufliche Rolle verändere und sie sich über Expertinnen und Experten ihres Faches hinaus auch als Technikexpertinnen und -experten sowie als Designerinnen und Designer verstehen (Schuck \& Mahler, 2018; Yildiz, 2018). Ebenso konnten sie mehr Autonomie und Kollaboration zwischen Schülerinnen und Schülern und positive Wirkungen der Smartphone-Nutzung auf deren soziales Leben beobachten. Insgesamt wird auch kritisch angemerkt, dass durchaus die Gefahr einer Überlastung sowohl des Lehrpersonals als auch der Schülerinnen und Schüler bestehe (Yildiz, 2018, S. 1).

18 Karaseva et al., 2018; Palkowitsch-Kühl, 2018; Tsouccas \& Meletiou-Mavrotheris, 2019 


\subsubsection{Theorie-Praxis-Beziehung}

Die Beziehung zwischen Bildungspraxis und Bildungsforschung wird in der Literatur ebenfalls thematisiert. Die Reflexion eines digitalgestützten Unterrichtsformates sei ein metakognitiver in Zyklen verlaufender Prozess (Michos, Hernández-Leo \& Albó, 2018, S. 1092). Lehrpersonen können in diesem Prozess von Wissenschaftlerinnen und Wissenschaftlern begleitet und unterstützt werden. Ein Design Based Research Ansatz ermögliche systematische Forschung, indem die Konzeption von Unterrichtsformaten mit Learning Analytics verknüpft werden (ebd.). Wenn das Lehrpersonal in bestimmten Phasen der Forschung einbezogen werde, dann führe das dazu, dass Reflexionsprozesse über Unterrichtsformate stärker formalisiert und sichtbarer gemacht werden können (ebd., S. 1093). „[...] the model of professional development [...] contributed to the integration of mobile devices into the school setting“ (Meletiou-Mavrotheris et al., 2019, S. 19) biete auch im Rahmen eines Action Research Ansatzes eine Möglichkeit für berufliche Weiterentwicklung des Lehrpersonals.

\subsubsection{Gelingensbedingungen und Hindernisse für Mediennutzung in der Unterrichtspraxis}

Zahlreiche Studien untersuchen Gelingensbedingungen und Hindernisse für eine digitalgestützte Unterrichtspraxis. Besonders in der neuesten Forschung wird die Bedeutung einer geeigneten technischen Infrastruktur betont. ${ }^{19} \mathrm{Zu}$ Beginn eines Prozesses der Medienintegration könne die Motivation des Lehrpersonals dadurch erheblich gesteigert werden, sich mit Medien im Unterricht zu beschäftigen. Wenn digitale Medien zur Verfügung stünden, hätten Lehrpersonen die Möglichkeit, sich damit vertraut $\mathrm{zu}$ machen und eigene Erfahrungen $\mathrm{zu}$ sammeln, was als „[...] most significant impact across all selected countries [...]“ (Drossel et al., 2017, S. 568) gelten kann. ${ }^{20}$ Die Bedeutung des Peer-Austausches könne für eine gelingende Medienintegration kaum hoch genug eingeschätzt werden. ${ }^{21}$

Darüber hinaus habe ein Raum des Ausprobierens und des Sammelns eigener Erfahrungen eine positive Wirkung auf die Entwicklung von Kompetenzen in der Nutzung digitaler Medien (Sauers \& McLeod, 2018, S. 907). Digitale Kompetenzen sind für eine digitalgestützte Unterrichtspraxis zwingend erforderlich und können durch berufliche Fort- und Weiterbildung vertieft und ausgebaut werden. Lern- und Anwendungsprozesse sollten miteinander verzahnt in Form situierten Lernens er-

19 Degirmenci, 2018; Kearney, Schuck, Aubusson \& Burke, 2018; Khlaif, 2018; Kilinc, Tarman \& Aydin, 2018; Pareja, 2018; Pima, 2019; Sauers \& McLeod, 2018

20 Siehe auch Howley, Wood \& Hough, 2011; Hsu, 2016; Kopcha, 2012; Willis, Lynch, Fradale \& Yeigh, 2019

21 Degirmenci, 2018; Kearney et al., 2018; Ntemngwa \& Oliver, 2018; Pima, 2019; Swertz, 2018 
folgen. ${ }^{22}$ Dabei sei wichtig, dass die Kompetenzentwicklung von Lehrpersonen über die Bedienung der Technik hinaus auch die pädagogisch-fachdidaktische Dimension umfasse. ${ }^{23}$ Es müsse für Lehrpersonen ein Zusammenhang zwischen Mediennutzung und pädagogischen Zielen erkennbar werden, die sich in einer digital geprägten Welt möglicherweise ändern: ${ }^{24}$ „[...] new technologies require new teacher roles, new pedagogies and new approaches to teacher training [...]" (Sipilä, 2014, S. 24 zitiert nach Makrakis, 2005) Eine der zentralen Gelingensbedingungen sei die individuelle Einschätzung der Lehrperson, wie souverän sie mit digitalen Medien umgehen könne und wie nützlich der Medieneinsatz für das Erreichen der eigenen Ziele sei. ${ }^{25}$ Insbesondere Lehrpersonen, die im Unterricht einen Schwerpunkt auf Problemlösungskompetenzen, Kreativität und selbstgesteuertes Lernen legen, sehen hier Potenziale und entscheiden sich häufiger für einen Medieneinsatz. ${ }^{26}$

Für diese Prozesse brauche es dreierlei: Zum einen bedarf es zeitlicher Ressourcen, um Lehrpersonen Möglichkeiten zu geben, digitale Medien auszuprobieren und technologische Entwicklungen zu verfolgen, mit Kolleginnen und Kollegen kollaborativ zu arbeiten und regelmäßige Evaluationszyklen durchführen zu können. ${ }^{27}$ Zum anderen sei es wichtig, Lehrpersonen über einen längeren Zeitraum administrativ und technisch durch eine eigens dafür ausgebildete Fachkraft oder eine digital-affine Lehrperson in dem Vorhaben zu unterstützen, digitalgestützte Unterrichtsformate zu entwickeln und durchzuführen. ${ }^{28}$ Schließlich bedarf es eines gewissen Grades an Schulautonomie, um Lehrpersonen den nötigen Freiraum zu schaffen, diese Veränderungsprozesse auf den Weg zu bringen (Eickelmann, 2011).

Mit Blick auf den Gesamtprozess wird für den Erfolg der Medienintegration ein innerer Zusammenhang zwischen folgenden Aspekten angenommen: „[...] erfolgreiches Handeln in Schule und Unterricht [erfordert] nicht nur Wissen und Können (in diesem Sinne Exzellenz), sondern auch Wollen [...] und Werte [... “" (Zierer, 2015, S. 10). Hier ist die Bedeutung einer gemeinsamen Policy sichtbar, der eine klar definierte Vision für eine Medienintegration in den Unterricht vorausgehe. ${ }^{29}$

Zusammenfassend können folgende zentrale Gelingensbedingungen in der untersuchten Literatur identifiziert werden: (1) Zugang zu einer geeigneten technischen Infrastruktur, (2) Möglichkeiten, Erfahrungen in der Nutzung digitaler Medien zu sammeln, (3) Peer-Austausch, (4) Kompetenz des Lehrpersonals in der technischen Nutzung und dem didaktischen Einsatz digitaler Medien, (5) Kompetenzentwicklung

22 Camilleri, 2018; Ekberg \& Gao, 2018; Khlaif, 2018; Kopcha, 2012; Li, Garza, Keicher \& Popov, 2018b; Voogt et al., 2011

23 Curtis, 2019; Degirmenci, 2018; Erduran \& Ince, 2018; Maisamari, Adikwu, Ogwuche \& Ikwoche, 2018; Pareja et al., 2018; Willis et al., 2019

24 Sipilä, 2014; Starkey, 2011

25 Eickelmann, 2011; Gregorcic, Etkina \& Planinsic, 2018; Pareja et al., 2018; Pima, 2019; Stockless, 2018; Willies et al., 2018

26 Pareja et al., 2018; Li, Yamaguchi \& Takada, 2018a

27 Hsu, 2016; Kale \& Grote, 2014; Kopcha, 2012

28 Erduran \& Ince, 2018; Khlaif, 2018; Kilinc et al., 2018; Ntemngwa \& Oliver, 2018

29 Ekberg \& Gao, 2018; Pareja et al., 2018 
bei Lehrpersonen durch situiertes Lernen, (6) zeitliche Ressourcen für Lehrpersonen sowie (7) administrativer und technischer Support.

\subsubsection{Korrelationen im Kontext der Medienintegration}

Einige Studien untersuchen Korrelationen der Mediennutzung, personalen Faktoren und Einstellungen sowie der Haltung von Lehrpersonen. Dabei können sowohl starke positive und auch negative Korrelationen zwischen den untersuchten Variablen festgestellt werden. ${ }^{30}$

In den Befunden kann nachgewiesen werden, dass ein Zusammenhang zwischen einer intensiven Mediennutzung und einer positiven Grundeinstellung der Lehrperson gegenüber digitalen Medien besteht. ${ }^{31}$

„Die Effekte ergeben sich für die wahrgenommenen Potenziale hinsichtlich (a) der Hilfe digitaler Medien bei der wirksameren Vertiefung und Verarbeitung von Informationen, (b) die Ermöglichung des Arbeitens auf einem den individuellen Lernbedürfnissen entsprechenden Niveau sowie (c) der Verbesserung der schulischen Leistungen durch den Einsatz digitaler Medien im Unterricht" (Lorenz \& Endberg, 2016, S. 222).

Einige interessante Befunde können auch bei der Untersuchung eines Zusammenhangs zwischen der pädagogischen Haltung der Lehrperson und dem Einsatz digitaler Medien ermittelt werden. (1) Abhängigkeiten zwischen pädagogischen Grundannahmen und einem Medieneinsatz wirken in beide Richtungen. (2) Der Einsatz digitaler Medien kann durch behavioristische pädagogische Grundhaltungen, Zeitdruck sowie durch die Sorge vor Kontrollverlust behindert oder ganz verhindert werden. Es kann angenommen werden, dass (3) ein Zusammenhang zwischen spezifischen Haltungen und der Art des Einsatzes digitaler Medien bestehe. Es gibt begründete Hinweise darauf, dass in lehrerzentrierten Szenarien digitale Medien stärker zum Erwerb von Qualifikationen eigesetzt werden. In Szenarien, die auf konstruktivistisch-pädagogischen Annahmen basieren, dienen sie eher als Tool zur Problemlösung. Gleichwohl bedarf es eines multidimensionalen Ansatzes und weiterer Forschung, um diesen Zusammenhang genauer zu untersuchen (Howley et al., 2011; Tondeur et al., 2017).

Der Befund, dass in schülerzentrierten Unterrichtspraktiken verstärkt digitale Medien zur Förderung zukunftsweisender Kompetenzen eingesetzt werden

30 Einige Studien haben auch schwache Korrelationen nachgewiesen, die aber hier nur dann Erwähnung finden, wenn sie eine besonders wichtige Bedeutung für das Lehrpersonal einnehmen.

31 Lee, Cerreto und Lee kommen in ihrer Studie über salient beliefs von Lehrpersonen auf Basis der Theory of Planned Behavior (Ajzen, 1985) zu dem Schluss, dass die Einstellung einer Lehrperson einen doppelt so hohen Einfluss auf die Mediennutzung hat wie die subjektive Wertehaltung und gar einen dreifach hohen Einfluss wie die Annahme, sein Verhalten und Handeln unter Kontrolle zu haben (2010, S. 158); Lorenz \& Endberg, 2016. 
wird nicht in allen Studien gleichermaßen ermittelt. ${ }^{32}$ Es kann auch nachgewiesen werden, dass die Überzeugung der Lehrperson, digitale Medien förderten den Lernprozess von Schülerinnen und Schülern, signifikanter für den Medieneinsatz sei. Ebenso wie ein hohes Selbstwirksamkeitsempfinden (Kaya \& Yazici, 2018) und Motivation sowie ein ausgeprägtes Selbstbewusstsein und eine gewisse Form von Extrovertiertheit der Lehrperson..$^{33}$ Auch stehe „[...] die Selbsteinschätzung der medienbezogenen Kompetenz in einem direkten Zusammenhang mit der unterrichtlichen Computernutzungshäufigkeit der Lehrkräfte [...]" (Drossel et al., 2018, S. 492). Ob Lehrpersonen über Kompetenzen im Sinne des TPACK verfügen, korreliere mit der Einstellung zu digitalen Medien sowie mit einigen demographischen Faktoren. Es scheint eine negative Korrelation zwischen digitalen Kompetenzen und hohem Alter sowie weiblichem Geschlecht zu bestehen. ${ }^{34}$

Es kann auch nachgewiesen werden, dass digitale Medien verstärkt eingesetzt werden, wenn es um allgemeine pädagogische Arbeit im Unterricht geht, wie Recherchen im Internet, die Erstellung multimedialer Präsentationen oder kollaboratives Arbeiten mit Mitschülerinnen und Mitschülern. Seltener werden digitale Medien in fachspezifischem Unterricht eingesetzt (Ward \& Parr, 2010). Die häufige Annahme, der Einsatz digitaler Medien führe zu Zeitersparnis, wird in einer Studie widerlegt, denn beide Variablen korrelieren signifikant negativ miteinander (Schweizer \& Horn, 2014).

Insgesamt kann mit einiger Vorsicht festgestellt werden, dass eine positive Haltung gegenüber digitalen Medien, lernerzentrierte Unterrichtspraktiken sowie ein hoher Grad an Technology Literacy, intrinsische Motivation und ein hohes Selbstwirksamkeitsempfinden in der Nutzung von Technik eine höhere Mediennutzung bewirken kann.

32 Liu, 2011; Petko, 2012; Ruggiero \& Mong, 2015

33 Camadan et al., 2018; Chang, 2012; Cheng \& Jang, 2018; Ciampa, 2014; Drossel et al., 2018; Efiliti \& Çoklar, 2019; Gil-Flores et al., 2017; Howard, 2013; Kaya \& Yazici, 2018; Schweizer \& Horn, 2014; Simsek \& Sarar, 2019; Suárez-Rodríguez, Almerich, Orellana \& Díaz-García, 2018. Einschränkend sei hier erwähnt, dass Zhang (2019) in den USA keine signifikante Korrelation zwischen Mediennutzung und Selbstwirksamkeitsempfinden ermitteln konnte; die Nutzung digitaler Medien werde verstärkt, wenn die Lehrperson nicht nur sporadisch in einer Klasse unterrichtet, sondern die Verantwortung z.B. in Form einer Klassenleitung übernommen hat (Petko, 2012, S. 1357).

34 Karaseva et al., 2018; Lai \& Lin, 2018; Lin, Tsai, Chai \& Lee, 2013; Semerci \& Aydin, 2018; Bingimlas (2018) stellt in seiner saudi-arabischen Studie fest, dass sowohl das Geschlecht als auch das Unterrichtsfach und die Berufserfahrung mit TPACK-Kompetenzen korrelieren. Dabei ist es wichtig zu erwähnen, dass sich der Unterschied zwischen Lehrerinnen und Lehrern nur in Bezug auf technische Kompetenzen beziehe. Insbesondere könne ein Unterschied zwischen beiden Geschlechtern zwischen technischen Kompetenzen und Berufserfahrung festgestellt werden. 


\section{Resümee}

\subsection{Aktuelle internationale Forschungsagenda zur Bedeutung der Digitalisierung für Lehrpersonen in der Schule}

Wenn digitale Medien in der Unterrichtspraxis Eingang finden, dann kann das auf sehr unterschiedliche Weise erfolgen. Das Spektrum erstreckt sich über einen Einsatz als Präsentationsmedium und als Ersatz klassischer analoger Materialien, um traditionelle Unterrichtsformate zu unterstützen und zu modernisieren. Es reicht bis zu pädagogisch-didaktischen Innovationen, die durch neue Formen des Wissenserwerbs und des kooperativen Arbeitens zu stärker selbstgesteuertem Lernen hinführen. In diesem Fall wird sich Lernen in der Schule verändern und einer zunehmend digital geprägten, globalisierten Welt Rechnung tragen, die erweiterte Formen der Kommunikation und der Zusammenarbeit über Klassenräume, Schulen und Staaten hinweg ermöglicht.

Zusammenfassend kann auf der Basis der vorangegangenen Untersuchung festgehalten werden, dass empirisch nachgewiesen ist, dass digitale Medien im beruflichen Alltag des Lehrpersonals bereits in der Kommunikation und als Präsentationswerkzeuge etabliert sind. Die Nutzung in der Unterrichtspraxis orientiert sich dabei allerdings selten an (medien-)pädagogischen Zielen, sodass digitale Medien eher als Tool und weniger als integraler Bestandteil des Lernens verstanden werden. Das korrespondiert mit dem Befund, dass das Lehrpersonal Computern und Smartboards mehrheitlich wohlwollend gegenübersteht. Dem World Wide Web des Internets oder sozialen Netzwerken wie Twitter und Facebook, die über den Klassenraum und die eigene Schule hinausweisen, jedoch mit Skepsis begegnet wird. Das scheint zu erklären, warum moderate bis gute technisch-instrumentelle Nutzungskompetenzen bei Lehrpersonen vorhanden sind, obwohl gleichzeitig selten digitalgestützte pädagogisch-didaktische Unterrichtsformate entwickelt und eingesetzt werden.

Spezifische personale Faktoren begünstigen innovative Transformationsprozesse in der Unterrichtspraxis. Lehrpersonen, die eher offene, extrovertierte, in gewisser Hinsicht auch risikofreudige Charaktereigenschaften besitzen und intrinsisch motiviert sind, werden in ihrem Unterricht eher technische und pädagogische Innovationen erproben als Lehrpersonen, die ein stärkeres Sicherheitsempfinden haben und deren Selbstbewusstsein schwächer ausgeprägt ist. Es kann empirisch nachgewiesen werden, dass Veränderungsprozesse dadurch unterstützt werden können, dass zeitliche Ressourcen geschaffen werden, die Möglichkeiten bieten, Erfahrungen zu sammeln und technische Entwicklungen mit verfolgen zu können. Dieses erhöht das Selbstwirksamkeitsempfinden von Lehrpersonen erheblich. Eine geeignete technische Ausstattung sowie technischer und administrativer Support sind wesentliche Erfolgsbedingungen für die Konzeption und Implementation digitalgestützter innovativer Unterrichtsformate. 
Mit dieser Entwicklung geht die Frage einher, über welche Kompetenzen Lehrpersonen verfügen müssen, um digitale Medien in die pädagogisch-didaktischen Überlegungen darüber einzubeziehen, wie ein Lehr-Lernziel erreicht werden kann. Ebenso stellt sich die Frage, wie die erweiterten Möglichkeiten der Kommunikation, Kooperation und Kollaboration genutzt werden können. An dieser Stelle kann es schwerlich ein zusammenfassendes Ergebnis geben, denn die Kompetenzmodelle weisen auf unterschiedliche normative Schwerpunktsetzungen hin, wie unsere zukünftige Welt beschaffen sein soll.

In der aktuellen Forschungsdebatte wird im Konnex der Kompetenzentwicklung von Lehrpersonen eine Alternative $\mathrm{zu}$ klassischen Lehrerfortbildungen diskutiert. Situierte Fort- und Weiterbildungsformate ermöglichen, Lern- und Anwendungsprozesse miteinander zu verzahnen, um medientechnische und pädagogische Grundlagen zu digitalgestützten Unterrichtsformaten zu erwerben. Sie sind langfristig angelegt, integrieren digitale Formate wie MOOCs oder Twitter und erfordern Reflexions- und Coachingphasen, die den individuellen Lernprozess ebenso fördern wie ein lebendiger Peer-Austausch.

\subsection{Fazit mit besonderem Blick auf die spezifisch deutsche Forschungsdebatte}

Ob und wie digitale Medien in der Unterrichtspraxis genutzt werden, hängt in hohem Maße von der einzelnen Lehrperson ab. Insofern ist es von besonderem Interesse, die bildungswissenschaftliche Forschung $\mathrm{zu}$ Einstellungen, der pädagogischen Haltung des Lehrpersonals, vorhandenen und noch notwendigen Kompetenzen sowie die Frage des Kompetenzerwerbs in Fort- und Weiterbildungen zu ermitteln. Das bietet eine empirisch gesicherte Basis für Entscheidungen, die in dieser sich verändernden Welt in der schulischen Bildungspraxis und im Rahmen bildungspolitischer Reformen notwendig sind.

Der Fokus auf neue Kompetenzen, die sich unmittelbar durch die Bedienung technischer Geräte ergeben, verkennt, dass die Digitalisierung die bisherige Unterrichtspraxis „[...] durchdringt und irritiert [...]“ (Kerres, 2020, S. 8). Daher lässt sich mit Blick auf aktuelle Forschungsergebnisse festhalten, dass eine Verschiebung von einem technischen $\mathrm{zu}$ einem pädagogischen Fokus in der Diskussion über Kompetenzen erforderlich ist. Nur auf diese Weise wird es möglich sein, in einen gesellschaftlichen, auch normativ geprägten, Verständigungsprozess zu treten, der weniger von einem Technikdeterminismus beherrscht ist, als von der Frage, welche Bedeutung Digitalisierungsprozesse für die Unterrichtspraxis und die Rolle der Lehrperson einnehmen sollen.

Bei deutschsprachigen Studien ist auffallend, dass ein Großteil keinen wissenschaftlichen Anspruch erhebt, sondern als Erfahrungsberichte, Good-Practice-Beispiele oder Handlungsempfehlungen angelegt sind. Lediglich $24 \%$ der Studien 
genügen wissenschaftlichen Gütekriterien. Mehrheitlich werden darin Einstellungen und Kompetenzen des Lehrpersonals untersucht, während in internationalen Studien die Untersuchungsfrage häufig breiter gefasst ist und nach Gelingensbedingungen für Medienintegration geforscht wird. Auffallend ist in diesem Zusammenhang auch, dass im deutschsprachigen Raum zur Kompetenzentwicklung traditionelle Lehrerfortbildungen das Mittel der Wahl sind. Im internationalen Kontext erfahren soziale Netzwerke wie Twitter oder Formate wie MOOCs in der Kompetenzentwicklung des Lehrpersonals größere Beachtung. Wird nur die Forschung seit 2018 in den Blick genommen, so kann festgestellt werden, dass häufiger als in den anderen Analysesträngen auf die Notwendigkeit einer geeigneten technischen Infrastruktur verwiesen wird.

\subsection{Perspektiven für weitere Forschung}

Es könnte ein interessanter Hinweis sein, dass in Studien, die im internationalen Kontext höchste Beachtung erfahren, unterbelichtet bleibt, welche Bedeutung gesellschaftliche Veränderungsprozesse für die Schule als Institution und das Lehrpersonal haben. Der Schwerpunkt aktueller Forschung liegt auf der Frage, was die Nutzung digitaler Medien in der Unterrichtspraxis begünstige. Dabei bleibt größtenteils unbeachtet, wie Lehrpersonen digitale Medien einsetzen und, ob durch deren Integration Implikationen für Unterrichtsformate und pädagogische Handlungspraktiken erkennbar sind.

Mehrheitlich wird in der aktuellen Forschung auf Kompetenzen und Fragen der Kompetenzentwicklung des Lehrpersonals abgehoben. Dabei verbleibt die Diskussion an der Stelle, Kompetenzen zu identifizieren, die additiv zu den bereits bestehenden Kompetenzen der Fachdisziplin und der Didaktik erworben werden sollen. Insbesondere in der deutschsprachigen Debatte wird auf sogenannte Medienkompetenzen Bezug genommen, die in Lehrerfortbildungen vermittelt werden. Die Engführung auf zusätzliche Kompetenzen verkennt, dass das Digitale unsere Lebenswelt durchdringt und Einfluss nimmt auf die Institution Schule, auf Bildungsinhalte und auf Lehrpersonen. Insofern sollte das Digitale integral gedacht und reflektierend betrachtet werden, welche Implikationen für Lehrpersonen damit verbunden sind.

„Neue, sogenannte digitale Kompetenzen sind [...] auffallend schwer zu begründen. Bei genauerer Betrachtung finden sich grundlegende Anforderungen, die mit einem Verweis auf den Bildungsbegriff und die Idee einer allgemeinen Bildung ebenso wie die grundlegenden Kompetenzen im Umgang mit Informationen und Medien gut beantwortet werden können“" (Kerres, 2020, S. 15). 
Weitere Forschung kann daran anschließen, Medienintegration als einen Schulentwicklungsprozess zu verstehen, der auf strategischen Überlegungen einer spezifischen Schulpolicy basiert. Wege der Teilhabe des Lehrpersonals an der Entwicklung einer Digitalstrategie sind bislang wenig thematisiert.

Es bleibt die Frage, wie Schule in einer digital geprägten Welt zukunftsfähig ist und welche Rolle Lehrpersonen darin einnehmen. Das wird in einer gemeinsamen Anstrengung von Bildungspraxis und Bildungswissenschaft zu beantworten sein. 
Unterrichtspraktiken, Erfahrungen und Einstellungen |

\section{Anhang}

\section{a. Forschungsdesign der Studien}

Tabelle 1: Analysestrang 1 (34 Studien)

\begin{tabular}{|c|c|c|c|c|c|c|c|c|c|c|c|c|c|c|c|}
\hline \multirow[t]{2}{*}{ Autor/in } & \multirow[t]{2}{*}{ Ort } & \multicolumn{5}{|c|}{ Forschungsdesign } & \multicolumn{8}{|c|}{ Art der Datenerhebung } & \multirow[t]{2}{*}{ Sample (n) } \\
\hline & & 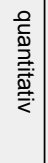 & 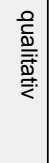 & 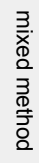 & 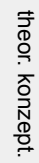 & 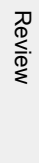 & 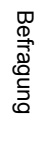 & 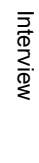 & 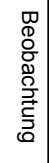 & 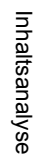 & 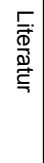 & $\begin{array}{l}\frac{3}{0} \\
\frac{0}{D} \\
\frac{D}{\bar{D}}\end{array}$ & 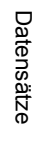 & 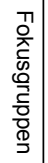 & \\
\hline Albion et al., 2015 & USA/Kanada & & $x$ & & & & & & & $\mathrm{x}$ & & & & & 4 Fallstudien \\
\hline $\begin{array}{l}\text { Alzaidiyeen et al., } \\
2010\end{array}$ & Asien & & $x$ & & & & $x$ & & & & & & & & $\begin{array}{l}50 \text { Lehr- } \\
\text { personen }\end{array}$ \\
\hline Anthony, 2012 & USA/Kanada & & $x$ & & & & & & $x$ & & & & & & $\begin{array}{l}2 \text { Lehr- } \\
\text { personen }\end{array}$ \\
\hline $\begin{array}{l}\text { Carpenter \& Krutka, } \\
2014\end{array}$ & USA/Kanada & & & $x$ & & & $x$ & & & & & & & & $\begin{array}{l}755 \text { Lehr- } \\
\text { personen }\end{array}$ \\
\hline $\begin{array}{l}\text { Carpenter \& Krutka, } \\
2015\end{array}$ & International & & & $\mathrm{x}$ & & & $x$ & & & & & & & & $\begin{array}{l}494 \text { Schul- } \\
\text { akteure }\end{array}$ \\
\hline Cassidy et al., 2012 & USA/Kanada & & $x$ & & & & & $x$ & & & & & & & $\begin{array}{l}17 \text { Schul- } \\
\text { akteure }\end{array}$ \\
\hline Chang, 2012 & Asien & $x$ & & & & & $\mathrm{x}$ & & & & & & & & $\begin{array}{l}1000 \\
\text { Lehrpersonen }\end{array}$ \\
\hline $\begin{array}{l}\text { Chiu \& Churchill, } \\
2016\end{array}$ & Asien & $x$ & & & & & $x$ & & & & & & & & $\begin{array}{l}62 \text { Lehr- } \\
\text { personen }\end{array}$ \\
\hline Ciampa, 2014 & USA/Kanada & & $x$ & & & & & & & $x$ & & & & & $1 \mathrm{Blog}$ \\
\hline Drossel et al., 2017 & International & $x$ & & & & & & & & & & & $x$ & & $\begin{array}{l}8920 \text { Pers. } \\
\text { Daten }\end{array}$ \\
\hline $\begin{array}{l}\text { Gil-Flores et al., } \\
2017\end{array}$ & Europa & $x$ & & & & & & & & & & & $x$ & & $\begin{array}{l}3339 \text { Pers. } \\
\text { Daten }\end{array}$ \\
\hline $\begin{array}{l}\text { Henriksen et al., } \\
2016\end{array}$ & Europa & & & & $x$ & & & & & & $x$ & & & & Literatur \\
\hline Howard, 2013 & $\begin{array}{l}\text { Australien/ } \\
\text { Neuseeland }\end{array}$ & & $x$ & & & & & $x$ & & & & & & & $\begin{array}{l}8 \text { Lehr- } \\
\text { personen }\end{array}$ \\
\hline Howley et al., 2011 & USA/Kanada & $x$ & & & & & $x$ & & & & & & & & $\begin{array}{l}514 \text { Lehr- } \\
\text { personen }\end{array}$ \\
\hline Hsu, 2016 & USA/Kanada & & & $\mathrm{x}$ & & & $x$ & $x$ & $x$ & & & & & & $\begin{array}{l}\text { 152/8/8 } \\
\text { Lehrpersonen }\end{array}$ \\
\hline Kale \& Goh, 2014 & USA/Kanada & $x$ & & & & & $x$ & & & & & & & & $\begin{array}{l}161 \text { Lehr- } \\
\text { personen }\end{array}$ \\
\hline Kereluik et al., 2013 & USA/Kanada & & & & & $x$ & & & & & & $x$ & & & 15 Modelle \\
\hline Kopcha, 2012 & USA/Kanada & & & $x$ & & & $x$ & $x$ & & & & & & & $\begin{array}{l}18 \text { Lehr- } \\
\text { personen }\end{array}$ \\
\hline Lee et al., 2010 & Asien & $x$ & & & & & $x$ & & & & & & & & $\begin{array}{l}34 \text { Lehr- } \\
\text { personen }\end{array}$ \\
\hline Lin et al., 2013 & Asien & $x$ & & & & & $x$ & & & & & & & & $\begin{array}{l}156 \text { Lehr- } \\
\text { personen }\end{array}$ \\
\hline Liu, 2011 & Asien & $x$ & & & & & $x$ & & & & & & & & $\begin{array}{l}1139 \text { Lehr- } \\
\text { personen }\end{array}$ \\
\hline
\end{tabular}


Bettina Waffner

\begin{tabular}{|c|c|c|c|c|c|c|c|c|c|c|c|c|c|c|c|}
\hline \multirow[t]{2}{*}{ Autor/in } & \multirow[t]{2}{*}{ Ort } & \multicolumn{5}{|c|}{ Forschungsdesign } & \multicolumn{8}{|c|}{ Art der Datenerhebung } & \multirow[t]{2}{*}{ Sample (n) } \\
\hline & & 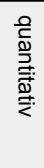 & 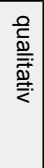 & 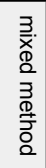 & 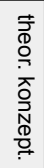 & 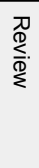 & 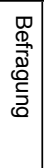 & 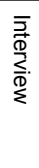 & 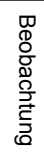 & 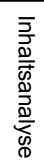 & 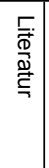 & $\begin{array}{l}3 \\
\frac{2}{0} \\
\frac{0}{\bar{D}}\end{array}$ & 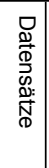 & 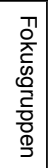 & \\
\hline $\begin{array}{l}\text { Lynch \& Redpath, } \\
2014\end{array}$ & $\begin{array}{l}\text { Australien/ } \\
\text { Neuseeland }\end{array}$ & & $x$ & & & & & $x$ & $x$ & & & & & & 1 Lehrperson \\
\hline Niess, 2011 & USA/Kanada & & & & $x$ & & & & & & $x$ & & & & Literatur \\
\hline Olofson et al., 2016 & USA/Kanada & & $x$ & & & & & $x$ & & & & & & & $\begin{array}{l}4 \text { Lehr- } \\
\text { personen }\end{array}$ \\
\hline $\begin{array}{l}\text { Ozdamli \& Uzunboy- } \\
\text { lu, } 2015\end{array}$ & Asien & $x$ & & & & & $x$ & & & & & & & & $\begin{array}{l}757 \text { Lehr- } \\
\text { personen }\end{array}$ \\
\hline Perrotta, 2013 & Europa & $x$ & & & & & $x$ & & & & & & & & $\begin{array}{l}683 \text { Lehr- } \\
\text { personen }\end{array}$ \\
\hline Petko, 2012 & Europa & $x$ & & & & & $x$ & & & & & & & & $\begin{array}{l}357 \text { Lehr- } \\
\text { personen }\end{array}$ \\
\hline $\begin{array}{l}\text { Ruggiero \& Mong, } \\
2015\end{array}$ & USA/Kanada & & & $x$ & & & $x$ & $x$ & & & & & & & $\begin{array}{l}\text { 1048/111 } \\
\text { Lehrpersonen }\end{array}$ \\
\hline Sipilä, 2014 & Europa & & & $x$ & & & $x$ & & & & & & & & $\begin{array}{l}292 \text { Lehr- } \\
\text { personen }\end{array}$ \\
\hline Starkey, 2011 & $\begin{array}{l}\text { Australien/ } \\
\text { Neuseeland }\end{array}$ & & $x$ & & & & & $x$ & & & & & & & $\begin{array}{l}6 \text { Lehr- } \\
\text { personen }\end{array}$ \\
\hline Tondeur et al., 2017 & International & & & & & $x$ & & & & $x$ & & & & & 14 Studien \\
\hline Voogt et al., 2011 & Europa & & $x$ & & & & & & & $x$ & & & & & $\begin{array}{l}\text { Tagungsdoku- } \\
\text { mentation }\end{array}$ \\
\hline Vrasidas, 2015 & Europa & & & $x$ & & & $x$ & $x$ & & & & & & & $\begin{array}{l}\text { 531/12 Lehr- } \\
\text { personen }\end{array}$ \\
\hline Wang et al., 2014 & USA/Kanada & & & $x$ & & & $x$ & $x$ & & & & & & & $\begin{array}{l}24 \text { Lehrper- } \\
\text { sonen }\end{array}$ \\
\hline Ward \& Parr, 2010 & $\begin{array}{l}\text { Australien/ } \\
\text { Neuseeland }\end{array}$ & $x$ & & & & & $x$ & & & & & & & & $\begin{array}{l}158 \text { Lehrper- } \\
\text { sonen }\end{array}$ \\
\hline
\end{tabular}


Unterrichtspraktiken, Erfahrungen und Einstellungen |

Tabelle 2: Analysestrang 2 (16 Studien)

\begin{tabular}{|c|c|c|c|c|c|c|c|c|c|c|c|c|c|}
\hline \multirow[t]{2}{*}{ Autor/in } & \multirow[t]{2}{*}{ Ort } & \multicolumn{4}{|c|}{$\begin{array}{l}\text { Forschungs- } \\
\text { design }\end{array}$} & \multicolumn{7}{|c|}{ Art der Datenerhebung } & \multirow[t]{2}{*}{ Sample (n) } \\
\hline & & 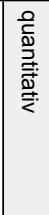 & 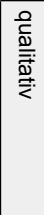 & 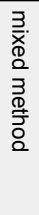 & 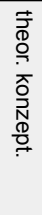 & 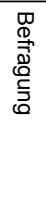 & 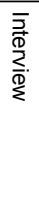 & 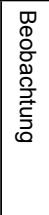 & 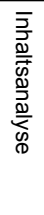 & 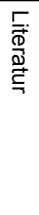 & 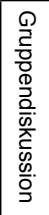 & 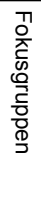 & \\
\hline Averbeck, 2014 & Deutschland & $x$ & & & & $x$ & & & & & & & 2431 Lehrpersonen \\
\hline Brüggemann, 2014 & Deutschland & & $x$ & & & & & & & & $x$ & & $\begin{array}{l}10 \text { Gruppen von } \\
\text { Lehrpersonen }\end{array}$ \\
\hline Drossel, 2018 & Deutschland & & $x$ & & & & $x$ & & & & & & 1210 Lehrpersonen \\
\hline Eickelmann, 2011 & Deutschland & & & $x$ & & $x$ & $x$ & & $x$ & & & & $\begin{array}{l}\text { 180/30 Lehr- } \\
\text { personen } \\
\text { Dokumente aus } 6 \\
\text { Schulen }\end{array}$ \\
\hline Hankmann, 2014 & Deutschland & $x$ & & & & $x$ & & & & & & & 502 Lehrpersonen \\
\hline Klemm, 2018 & Deutschland & & & & $x$ & & & & & $x$ & & & - \\
\hline Knaus, 2012 & Deutschland & $x$ & & & & & & & $x$ & & & & $\begin{array}{l}250 \text { Dokumenta- } \\
\text { tionen }\end{array}$ \\
\hline $\begin{array}{l}\text { Lorenz \& Endberg, } \\
2016\end{array}$ & Deutschland & $x$ & & & & $x$ & & & & & & & 1250 Lehrpersonen \\
\hline Ludewig et al., 2013 & Deutschland & & & $x$ & & $x$ & $x$ & & & & & $x$ & $\begin{array}{l}\text { 194/21/3 Lehr- } \\
\text { personen und } \\
\text { Schulleitungen }\end{array}$ \\
\hline $\begin{array}{l}\text { Palkowitsch-Kühl, } \\
2018\end{array}$ & Deutschland & $x$ & & & & $x$ & & & & & & & 132 Lehrpersonen \\
\hline $\begin{array}{l}\text { Schiefner-Rohs, } \\
2014\end{array}$ & Deutschland & & $x$ & & & & & & $x$ & & & & $\begin{array}{l}3 \text { Blogs von Lehr- } \\
\text { personen }\end{array}$ \\
\hline Schrammel, 2010 & Deutschland & & & & $x$ & & & & & $x$ & & & - \\
\hline Schulze, 2015 & Deutschland & & & & $x$ & & & & & $x$ & & & - \\
\hline $\begin{array}{l}\text { Schweizer \& Horn, } \\
2014\end{array}$ & Deutschland & $x$ & & & & $x$ & & & & & & & 99 Lehrpersonen \\
\hline Swertz, 2018 & Österreich & & & $x$ & & & $x$ & $x$ & & & & & 15 Schulen \\
\hline Zierer, 2015 & Deutschland & & & & $x$ & & & & & $x$ & & & - \\
\hline
\end{tabular}


Bettina Waffner

Tabelle 3: Analysestrang 3 (74 Studien)

\begin{tabular}{|c|c|c|c|c|c|c|c|c|c|c|c|c|c|c|c|c|c|c|}
\hline \multirow[t]{2}{*}{ Autor/in } & \multirow[t]{2}{*}{ Ort } & \multicolumn{4}{|c|}{$\begin{array}{l}\text { Forschungs- } \\
\text { design }\end{array}$} & \multicolumn{12}{|c|}{ Art der Datenerhebung } & \multirow[t]{2}{*}{ Sample (n) } \\
\hline & & 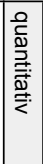 & 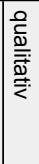 & 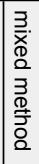 & 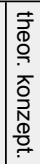 & 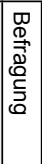 & 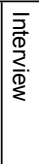 & 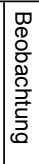 & 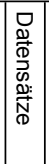 & 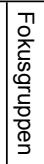 & 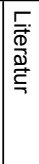 & 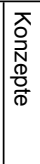 & 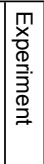 & 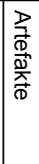 & 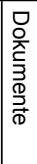 & $\begin{array}{l}0 \\
0 \\
\frac{0}{\infty} \\
\omega\end{array}$ & \begin{tabular}{|l}
$\frac{2}{8}$ \\
$\frac{1}{0}$
\end{tabular} & \\
\hline $\begin{array}{l}\text { Abubakar \& Salmanu, } \\
2018\end{array}$ & Afrika & $x$ & & & & $x$ & & & & & & & & & & & & $\begin{array}{l}300 \text { Lehr- } \\
\text { personen }\end{array}$ \\
\hline Andic et al., 2018 & Europa & & $x$ & & & & $x$ & & & & $x$ & & $x$ & & & & & $\begin{array}{l}270 \text { Lehr- } \\
\text { personen }\end{array}$ \\
\hline Biçak, 2019 & Asien/Türkei & & $x$ & & & & $x$ & & & & & & & & & & & $\begin{array}{l}10 \text { Lehr- } \\
\text { personen }\end{array}$ \\
\hline Bingimlas, 2019 & Asien/Türkei & $x$ & & & & $x$ & & & $x$ & & & & & & & & & $\begin{array}{l}243 \text { Lehr- } \\
\text { personen }\end{array}$ \\
\hline Buliva, 2018 & Afrika & $x$ & & & & $x$ & & & & & & & & & & & & $\begin{array}{l}100 \text { Lehr- } \\
\text { personen }\end{array}$ \\
\hline Camadan et al., 2018 & Asien/Türkei & $x$ & & & & $x$ & & & & & & & & & & & & $\begin{array}{l}425 \text { Lehr- } \\
\text { personen }\end{array}$ \\
\hline Camilleri, 2018 & Europa & & $x$ & & & & $x$ & & & & & & & & & & & $\begin{array}{l}31 \text { Lehr- } \\
\text { personen }\end{array}$ \\
\hline $\begin{array}{l}\text { Castaño-Muñoz et } \\
\text { al., } 2018\end{array}$ & Europa & $x$ & & & & $x$ & & & & & & & & & & & & $\begin{array}{l}2486 \text { (Nicht-) } \\
\text { Lehrpersonen }\end{array}$ \\
\hline Chen \& Jang, 2018 & Asien/Türkei & $x$ & & & & $x$ & & & & & & & & & & & & \begin{tabular}{|l|}
386 Lehr- \\
personen \\
\end{tabular} \\
\hline Curtis, 2019 & USA/Kanada & & & $x$ & & $x$ & $x$ & & & & & & & & & & & $\begin{array}{l}\text { 78/14 Lehr- } \\
\text { personen }\end{array}$ \\
\hline Daher et al., 2018 & USA/Kanada & & $x$ & & & & $x$ & $x$ & & & & & & & & & & 5 Lehrpersonen \\
\hline $\begin{array}{l}\text { DeCoito \& Richard- } \\
\text { son, } 2018\end{array}$ & USA/Kanada & & & $x$ & & $x$ & $x$ & & & & & & & & & & & \begin{tabular}{|l|}
74 Lehr- \\
personen
\end{tabular} \\
\hline Degirmenci, 2018 & Asien/Türkei & & $x$ & & & & $x$ & & & & & & & & & & & \begin{tabular}{|l|}
15 Lehr- \\
personen
\end{tabular} \\
\hline Drossel et al., 2018 & USA/Kanada & $x$ & & & & $x$ & & & & & & & & & & & & \begin{tabular}{|l}
1210 Lehr- \\
personen
\end{tabular} \\
\hline Efilti \& Çoklar, 2019 & Asien/Türkei & $x$ & & & & $x$ & & & & & & & & & & & & $\begin{array}{l}228 \text { Lehr- } \\
\text { personen }\end{array}$ \\
\hline Ekberg \& Gao, 2018 & Europa & & $x$ & & & & $x$ & & & & & & & & & & & 4 Lehrpersonen \\
\hline Erduran \& Ince, 2018 & Asien/Türkei & & $x$ & & & & $x$ & & & $x$ & & $x$ & & & & & & 5 Lehrpersonen \\
\hline $\begin{array}{l}\text { Forkosh-Baruch \& } \\
\text { Hershkovitz, } 2019 \\
\end{array}$ & Asien/Türkei & & $x$ & & & $x$ & & & & & & & & & & & & \begin{tabular}{|l|}
180 Lehr- \\
personen \\
\end{tabular} \\
\hline Fransson et al., 2019 & Europa & & $x$ & & & & $x$ & $x$ & & & & & & & & & & 2 Lehrpersonen \\
\hline Frumin et al., 2018 & USA/Kanada & & & $x$ & & $x$ & $x$ & & & & & & & & & & & $\begin{array}{l}34 \text { Lehr- } \\
\text { personen }\end{array}$ \\
\hline Gregorcic et al., 2018 & Europa & & $x$ & & & & $x$ & $x$ & & & & & & & & & $\mathrm{x}$ & 2 Lehrpersonen \\
\hline Herro et al., 2018 & USA/Kanada & & $x$ & & & & & $x$ & & & & & & & $x$ & & & \begin{tabular}{|l|}
21 Lehr- \\
personen \\
\end{tabular} \\
\hline Holmberg et al., 2018 & Europa & & $x$ & & & & $x$ & & & $x$ & & & & & $x$ & & & 8 Lehrpersonen \\
\hline $\begin{array}{l}\text { Howlett \& Waemusa, } \\
2018\end{array}$ & Asien/Türkei & $x$ & & & & $x$ & & & & & & & & & & & & \begin{tabular}{|l}
55 Lehr- \\
personen
\end{tabular} \\
\hline Islahi \& Nasrin, 2019 & Indien & $x$ & & & & $x$ & & & & & & & & & & & & \begin{tabular}{|l}
482 Lehr- \\
personen
\end{tabular} \\
\hline Jong et al., 2018 & USA/Kanada & $x$ & & & & $x$ & & & & & & & & & & & & \begin{tabular}{|l|}
273 Lehr- \\
personen
\end{tabular} \\
\hline Kali et al., 2018 & Asien/Türkei & & $x$ & & & & $x$ & & & & & & & $x$ & $x$ & & & $\begin{array}{l}41 \text { Lehr- } \\
\text { personen }\end{array}$ \\
\hline
\end{tabular}


Unterrichtspraktiken, Erfahrungen und Einstellungen |

\begin{tabular}{|c|c|c|c|c|c|c|c|c|c|c|c|c|c|c|c|c|c|c|}
\hline \multirow[t]{2}{*}{ Autor/in } & \multirow[t]{2}{*}{ Ort } & \multicolumn{4}{|c|}{$\begin{array}{l}\text { Forschungs- } \\
\text { design }\end{array}$} & \multicolumn{12}{|c|}{ Art der Datenerhebung } & \multirow[t]{2}{*}{ Sample (n) } \\
\hline & & 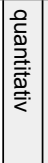 & 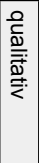 & 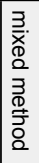 & 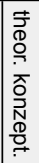 & 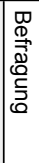 & 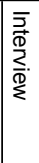 & 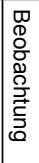 & 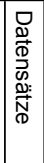 & 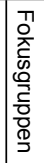 & 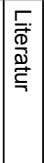 & 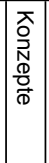 & 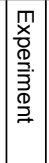 & 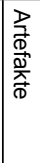 & 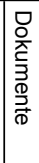 & 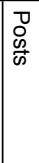 & $\frac{\widehat{\alpha}}{\mathrm{d}}$ & \\
\hline Karaseva et al., 2018 & Europa & & $x$ & & & & $x$ & & & & & & & & & & & \begin{tabular}{|l|}
26 Lehr- \\
personen
\end{tabular} \\
\hline Kaya \& Yazici, 2018 & Asien/Türkei & $\mathrm{x}$ & & & & $x$ & & & & & & & & & & & & $\begin{array}{l}101 \text { Lehr- } \\
\text { personen }\end{array}$ \\
\hline Kearney et al., 2018 & Australien & & & $x$ & & $x$ & & & & $x$ & & & & & & & & $\begin{array}{l}200 \text { Lehr- } \\
\text { personen }\end{array}$ \\
\hline Khlaif, 2018 & Asien/Türkei & & $x$ & & & & $x$ & & & & & $x$ & & & & & & $\begin{array}{l}15 \text { Lehr- } \\
\text { personen }\end{array}$ \\
\hline Kilinc et al., 2018 & Asien/Türkei & $x$ & & & & $x$ & & & & & & & & & & & & $\begin{array}{l}171 \text { Lehr- } \\
\text { personen }\end{array}$ \\
\hline Klemm, 2018 & USA/Kanada & & & & $x$ & & & & & & $x$ & & & & & & & - \\
\hline Koh, 2019 & Asien/Türkei & & $x$ & & & $x$ & & & & & & & & & & & & $\begin{array}{l}47 \text { Lehr- } \\
\text { personen }\end{array}$ \\
\hline $\begin{array}{l}\text { Koukis \& Jimoyiannis, } \\
2019\end{array}$ & Europa & & & $x$ & & $x$ & & & $x$ & & & & & & & & & $\begin{array}{l}327 \text { Lehr- } \\
\text { personen }\end{array}$ \\
\hline Kul, 2018 & Asien/Türkei & & $x$ & & & & $x$ & $x$ & & & & & & & & & & 6 Lehrpersonen \\
\hline Lai \& Lin, 2018 & Asien/Türkei & $x$ & & & & $x$ & & & & & & & & & & & & $\begin{array}{l}408 \text { Lehr- } \\
\text { personen }\end{array}$ \\
\hline Leem \& Sung, 2019 & Asien/Türkei & $x$ & & & & $x$ & & & & & & & & & & & & $\begin{array}{l}768 \text { Lehr- } \\
\text { personen }\end{array}$ \\
\hline Li et al., 2018a & Asien/Türkei & $x$ & & & & & & & $x$ & & & & & & & & & $\begin{array}{l}838 \text { Lehr- } \\
\text { personen }\end{array}$ \\
\hline Li et al., 2018b & USA/Kanada & $x$ & & & & $x$ & & & & & & & & & & & & \begin{tabular}{|l|}
928 Lehr- \\
personen \\
\end{tabular} \\
\hline Looi et al., 2018 & Afrika & & & $x$ & & & $x$ & $x$ & & & & & & & $x$ & & & 2 Lehrpersonen \\
\hline $\begin{array}{l}\text { Maisamari et al., } \\
2018\end{array}$ & Afrika & $x$ & & & & $x$ & & & & & & & & & & & & \begin{tabular}{|l|}
140 Lehr- \\
personen \\
\end{tabular} \\
\hline $\begin{array}{l}\text { Meletiou-Mavrotheris } \\
\text { et al., } 2019\end{array}$ & Europa & & & $x$ & & $x$ & $x$ & & & & & & & & & & & 6 Lehrpersonen \\
\hline Mesfin et al., 2018 & Afrika & $x$ & & & & $x$ & & & & & & & & & & & & \begin{tabular}{|l|}
14 Lehr- \\
personen
\end{tabular} \\
\hline Michos et al., 2018 & Europa & & & $x$ & & $x$ & $x$ & & & $x$ & & & & & $x$ & & & \begin{tabular}{|l|}
33 Lehr- \\
personen
\end{tabular} \\
\hline $\begin{array}{l}\text { Mtebe \& Raphael, } \\
2018\end{array}$ & Afrika & & & $x$ & & $x$ & & $x$ & & & & & & & & & & $\begin{array}{l}132 \text { Lehr- } \\
\text { personen }\end{array}$ \\
\hline Muhaimin et al., 2019 & Asien/Türkei & & & $x$ & & $x$ & $x$ & & & & & & & & & & & \begin{tabular}{|l|}
$356 / 8$ Lehr- \\
personen
\end{tabular} \\
\hline Muslem et al., 2018 & Asien/Türkei & & & $x$ & & $x$ & $x$ & & & & & & & & & & & $\begin{array}{l}26 \text { Lehr- } \\
\text { personen }\end{array}$ \\
\hline Mwila, 2018 & Afrika & $x$ & & & & $x$ & $x$ & $x$ & & & & & & & & & & $\begin{array}{l}100 \text { Lehr- } \\
\text { personen }\end{array}$ \\
\hline Nami et al., 2018 & Asien/Türkei & & $x$ & & & & & & & & & & & & & $x$ & & 5 Lehrpersonen \\
\hline $\begin{array}{l}\text { Ntemngwa \& Oliver, } \\
2018\end{array}$ & USA/Kanada & & $x$ & & & & $x$ & $x$ & & & & & & & & & & 5 Lehrpersonen \\
\hline Nugent et al., 2018 & USA/Kanada & $x$ & & & & $x$ & & & & & & & & & & & & $\begin{array}{l}124 \text { Lehr- } \\
\text { personen }\end{array}$ \\
\hline $\begin{array}{l}\text { Palkowitsch-Kühl, } \\
2018\end{array}$ & Europa & $x$ & & & & $x$ & & & & & & & & & & & & \begin{tabular}{|l|}
132 Lehr- \\
personen
\end{tabular} \\
\hline $\begin{array}{l}\text { Pareja Roblin et al., } \\
2018\end{array}$ & Europa & & $x$ & & & & $x$ & $x$ & & & & & & & & & & 9 Lehrpersonen \\
\hline
\end{tabular}


Bettina Waffner

\begin{tabular}{|c|c|c|c|c|c|c|c|c|c|c|c|c|c|c|c|c|c|c|}
\hline \multirow[t]{2}{*}{ Autor/in } & \multirow[t]{2}{*}{ Ort } & \multicolumn{4}{|c|}{$\begin{array}{l}\text { Forschungs- } \\
\text { design }\end{array}$} & \multicolumn{12}{|c|}{ Art der Datenerhebung } & \multirow[t]{2}{*}{ Sample (n) } \\
\hline & & 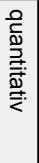 & 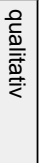 & 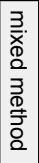 & 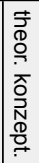 & 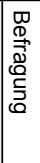 & 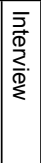 & 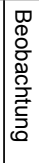 & 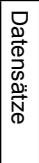 & 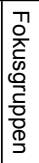 & 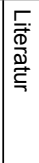 & 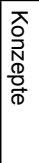 & 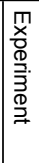 & 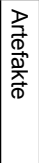 & 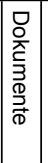 & $\begin{array}{l}0 \\
0 \\
\frac{0}{\omega} \\
\omega\end{array}$ & $\begin{array}{l}\widehat{\alpha} \\
\frac{\alpha}{0} \\
\end{array}$ & \\
\hline $\begin{array}{l}\text { Pérez-Paredes et al., } \\
2018\end{array}$ & Europa & $x$ & & & & $x$ & & & & & & & & & & & & $\begin{array}{l}230 \text { Lehr- } \\
\text { personen }\end{array}$ \\
\hline Pima, 2019 & Afrika & $x$ & & & & $x$ & & & & & & & & & & & & $\begin{array}{l}111 \text { Lehr- } \\
\text { personen }\end{array}$ \\
\hline Rana et al., 2018 & Indien & & $x$ & & & & $x$ & $x$ & & & & & & & & & & $\begin{array}{l}\text { 16/7 Lehr- } \\
\text { personen }\end{array}$ \\
\hline $\begin{array}{l}\text { Reichert \& Mouza, } \\
2018\end{array}$ & USA/Kanada & & $x$ & & & & $\mathrm{X}$ & $x$ & & & & $x$ & & & & & & 9 Lehrpersonen \\
\hline Rosell-Aguilar, 2018 & Europa & & $x$ & & & $\mathrm{x}$ & $\mathrm{x}$ & & & & & & & & & & & $\begin{array}{l}116 \text { Lehr- } \\
\text { personen }\end{array}$ \\
\hline Sarac, 2018 & Asien/Türkei & & $x$ & & & $\mathrm{x}$ & & & & & & & & & & & & $\begin{array}{l}32 \text { Lehr- } \\
\text { personen }\end{array}$ \\
\hline $\begin{array}{l}\text { Sauers \& McLeod, } \\
2018\end{array}$ & USA/Kanada & $x$ & & & & $x$ & & & $x$ & & & & & & & & & $\begin{array}{l}922 \text { Lehr- } \\
\text { personen }\end{array}$ \\
\hline $\begin{array}{l}\text { Schuck \& Maher, } \\
2018\end{array}$ & Australien & & $x$ & & & & $x$ & $x$ & & & & & & & $x$ & & & $\begin{array}{l}11 \text { Lehr- } \\
\text { personen }\end{array}$ \\
\hline $\begin{array}{l}\text { Semerci \& Aydin, } \\
2018\end{array}$ & Asien/Türkei & $x$ & & & & $x$ & & & & & & & & & & & & $\begin{array}{l}353 \text { Lehr- } \\
\text { personen }\end{array}$ \\
\hline Sheffield et al., 2018 & Australien & & $x$ & & & $x$ & & & & & & & & & & & & $\begin{array}{l}28 \text { Lehr- } \\
\text { personen }\end{array}$ \\
\hline $\begin{array}{l}\text { Simsek \& Sarsar, } \\
2019\end{array}$ & Asien/Türkei & $x$ & & & & $x$ & & & & & & & & & & & & $\begin{array}{l}387 \text { Lehr- } \\
\text { personen }\end{array}$ \\
\hline Stockless, 2018 & USA/Kanada & $x$ & & & & $x$ & & & & & & & & & & & & $\begin{array}{l}105 \text { Lehr- } \\
\text { personen }\end{array}$ \\
\hline $\begin{array}{l}\text { Suárez-Rodríguez et } \\
\text { al., } 2018\end{array}$ & Europa & $x$ & & & & & & & $x$ & & & & & & & & & $\begin{array}{l}1095 \text { Lehr- } \\
\text { personen }\end{array}$ \\
\hline Swertz, 2018 & Afrika & & & $x$ & & & $x$ & $x$ & & & & & & & & & & 15 Schulen \\
\hline $\begin{array}{l}\text { Tsouccas \& Meletiou- } \\
\text { Mavrotheris, } 2019\end{array}$ & Europa & & $x$ & & & $\mathrm{x}$ & $x$ & $x$ & & & & & & $x$ & & & & 6 Lehrpersonen \\
\hline $\begin{array}{l}\text { van de Oudeweete- } \\
\text { ring \& Voogt, } 2018\end{array}$ & Europa & $x$ & & & & & & & $x$ & & & & & & & & & $\begin{array}{l}2804 \text { Lehr- } \\
\text { personen }\end{array}$ \\
\hline Wake \& Mills, 2018 & USA/Kanada & & & $x$ & & $x$ & & & & & & & & & & & & $\begin{array}{l}57 \text { Lehr- } \\
\text { personen }\end{array}$ \\
\hline Willis et al., 2019 & Australien & $x$ & & & & $x$ & & & & & & & & & & & & $\begin{array}{l}45 \text { Lehr- } \\
\text { personen }\end{array}$ \\
\hline Yildiz, 2018 & Asien/Türkei & $x$ & & & & $x$ & & & & & & & & & & & & $\begin{array}{l}105 \text { Lehr- } \\
\text { personen }\end{array}$ \\
\hline Zhang, 2019 & USA/Kanada & & & $x$ & & $x$ & & & & & & & & & & & & $\begin{array}{l}286 \text { Lehr- } \\
\text { personen }\end{array}$ \\
\hline
\end{tabular}


Unterrichtspraktiken, Erfahrungen und Einstellungen |

\section{b. Forschungsthemen der Studien}

Tabelle 4: Untersuchungsgegenstand des Analysestranges 1 (34 Studien)

\begin{tabular}{|c|c|c|c|c|c|c|c|c|c|c|c|c|c|c|}
\hline \multirow[t]{3}{*}{ Autor/in } & \multicolumn{14}{|c|}{ Untersuchungsgegenstand } \\
\hline & \multicolumn{9}{|c|}{ Korrelationen } & \multicolumn{2}{|c|}{$\begin{array}{l}\text { Kompeten- } \\
\text { zen in der } \\
\text { digital ge- } \\
\text { prägten Welt }\end{array}$} & \multirow[t]{2}{*}{$\begin{array}{l}\text { Gelingens- } \\
\text { bedingun- } \\
\text { gen für } \\
\text { Medien- } \\
\text { integration }\end{array}$} & \multicolumn{2}{|c|}{$\begin{array}{l}\text { Einstellung } \\
\text { und Haltung } \\
\text { des Lehrper- } \\
\text { sonals }\end{array}$} \\
\hline & 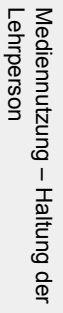 & 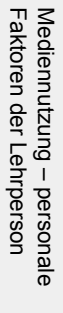 & 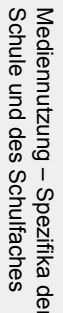 & 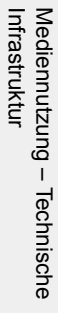 & 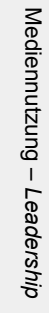 & 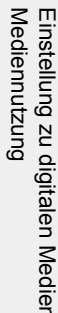 & 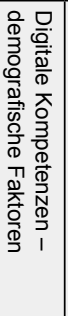 & 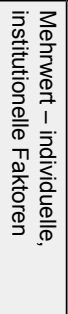 & 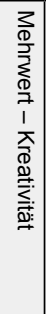 & 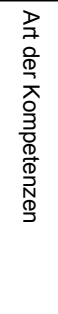 & 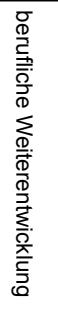 & & 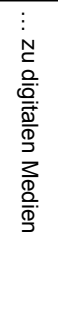 & 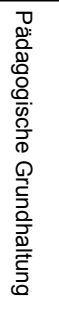 \\
\hline $\begin{array}{l}\text { Albion et al., } \\
2015\end{array}$ & & & & & & & & & & & $x$ & & & \\
\hline $\begin{array}{l}\text { Alzaidiyeen } \\
\text { et al., } 2010\end{array}$ & & & & & & & & & & & & & $x$ & \\
\hline Anthony, 2012 & & & & & & & & & & & & $x$ & & \\
\hline $\begin{array}{l}\text { Carpenter \& } \\
\text { Krutka, } 2014\end{array}$ & & & & & & & & & & & $x$ & & & \\
\hline $\begin{array}{l}\text { Carpenter \& } \\
\text { Krutka, } 2015\end{array}$ & & & & & & & & & & & $x$ & & & \\
\hline $\begin{array}{l}\text { Cassidy et al., } \\
2012\end{array}$ & & & & & & & & & & $\mathrm{X}$ & & & & \\
\hline Chang, 2012 & & $x$ & & & $x$ & & & & & & & & & \\
\hline $\begin{array}{l}\text { Chiu \& } \\
\text { Churchill, } \\
2016\end{array}$ & & & $\mathrm{x}$ & & & $\mathrm{X}$ & & & & & & & & \\
\hline Ciampa, 2014 & $\mathrm{x}$ & & & & & & & & & & & & & \\
\hline $\begin{array}{l}\text { Drossel et al., } \\
2017\end{array}$ & & & & & & & & & & & & $x$ & & \\
\hline $\begin{array}{l}\text { Gil-Flores et } \\
\text { al., } 2017\end{array}$ & & $x$ & & $x$ & & & & & & & & & & \\
\hline $\begin{array}{l}\text { Henriksen et } \\
\text { al., } 2016\end{array}$ & & & & & & & & & $x$ & & & & & \\
\hline Howard, 2013 & & $x$ & & & & & & & & & & & & \\
\hline $\begin{array}{l}\text { Howley et al., } \\
2011\end{array}$ & $x$ & & & & & & & & & & & $x$ & & \\
\hline Hsu, 2016 & & & & & & & & & & & & $x$ & $x$ & \\
\hline $\begin{array}{l}\text { Kale \& Goh, } \\
2014\end{array}$ & & & & & & & & & & & & $x$ & & \\
\hline $\begin{array}{l}\text { Kereluik et al., } \\
2013\end{array}$ & & & & & & & & & & $x$ & & & & \\
\hline Kopcha, 2012 & & & & & & & & & & & & $x$ & & \\
\hline $\begin{array}{l}\text { Lee et al., } \\
2010\end{array}$ & $x$ & & & & & & & & & & & & & \\
\hline $\begin{array}{l}\text { Lin et al., } \\
2013\end{array}$ & & & & & & & $x$ & & & & & & & \\
\hline Liu, 2011 & $x$ & & & & & & & & & & & & & \\
\hline
\end{tabular}




\begin{tabular}{|c|c|c|c|c|c|c|c|c|c|c|c|c|c|c|}
\hline \multirow[t]{3}{*}{ Autor/in } & \multicolumn{14}{|c|}{ Untersuchungsgegenstand } \\
\hline & \multicolumn{9}{|c|}{ Korrelationen } & \multicolumn{2}{|c|}{$\begin{array}{l}\text { Kompeten- } \\
\text { zen in der } \\
\text { digital ge- } \\
\text { prägten Welt }\end{array}$} & \multirow[t]{2}{*}{$\begin{array}{l}\text { Gelingens- } \\
\text { bedingun- } \\
\text { gen für } \\
\text { Medien- } \\
\text { integration }\end{array}$} & \multicolumn{2}{|c|}{$\begin{array}{l}\text { Einstellung } \\
\text { und Haltung } \\
\text { des Lehrper- } \\
\text { sonals }\end{array}$} \\
\hline & 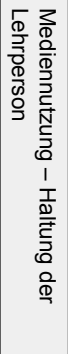 & 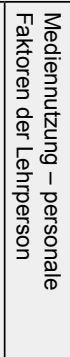 & 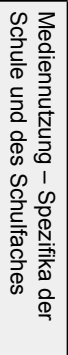 & 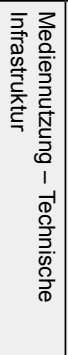 & 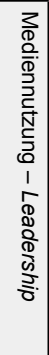 & 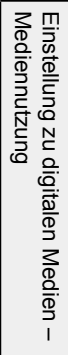 & 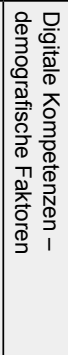 & 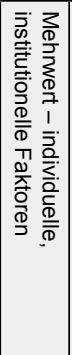 & 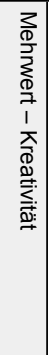 & 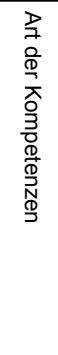 & 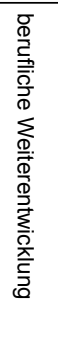 & & 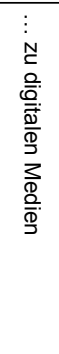 & 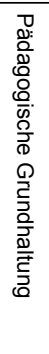 \\
\hline $\begin{array}{l}\text { Lynch \& Red- } \\
\text { path, } 2014\end{array}$ & & & & & & & & & & & & & & $x$ \\
\hline Niess, 2011 & & & & & & & & & & & $\mathrm{X}$ & & & \\
\hline $\begin{array}{l}\text { Olofson et al., } \\
2016\end{array}$ & & & & & & & & & & & $x$ & & & \\
\hline $\begin{array}{l}\text { Ozdamli \& } \\
\text { Uzunboylu, } \\
2015\end{array}$ & & & & & & & & & & $x$ & & & $x$ & \\
\hline Perrotta, 2013 & & & & & & & & $x$ & & & & & & \\
\hline Petko, 2012 & $x$ & $x$ & & $x$ & & & & & & & & & & \\
\hline $\begin{array}{l}\text { Ruggiero \& } \\
\text { Mong, } 2015\end{array}$ & $\mathrm{x}$ & & & & & & & & & & & & & \\
\hline Sipilä, 2014 & & & & & & & & & & $x$ & & $x$ & & \\
\hline Starkey, 2011 & & & & & & & & & & & & $x$ & & \\
\hline $\begin{array}{l}\text { Tondeur et al., } \\
2017\end{array}$ & $\mathrm{x}$ & & & & & & & & & & & & & \\
\hline $\begin{array}{l}\text { Voogt et al., } \\
2011\end{array}$ & & & & & & & & & & & & $x$ & & \\
\hline $\begin{array}{l}\text { Vrasidas, } \\
2015\end{array}$ & & & & & & & & & & & & $x$ & & \\
\hline $\begin{array}{l}\text { Wang et al., } \\
2014\end{array}$ & & & & & & & & & & & $\mathrm{x}$ & & & \\
\hline $\begin{array}{l}\text { Ward \& Parr, } \\
2010\end{array}$ & & & $x$ & & & & & & & & & & & \\
\hline
\end{tabular}


Unterrichtspraktiken, Erfahrungen und Einstellungen

Tabelle 5: Untersuchungsgegenstand des Analysestranges 2 (16 Studien)

\begin{tabular}{|c|c|c|c|c|c|c|c|c|}
\hline \multirow[t]{2}{*}{ Autor/in } & \multicolumn{2}{|c|}{$\begin{array}{l}\text { Kompetenzen in } \\
\text { der digital ge- } \\
\text { prägten Welt }\end{array}$} & \multicolumn{3}{|c|}{ Korrelationen } & \multirow{2}{*}{ 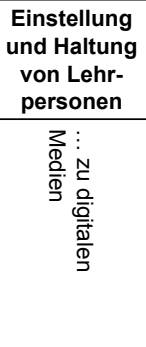 } & \multirow[t]{2}{*}{$\begin{array}{l}\text { Gelingensbe- } \\
\text { dingungen für } \\
\text { Medien- } \\
\text { integration }\end{array}$} & \multirow[t]{2}{*}{$\begin{array}{l}\text { Art der } \\
\text { Medien- } \\
\text { nutzung }\end{array}$} \\
\hline & 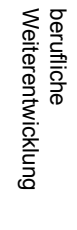 & 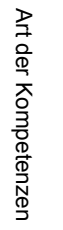 & 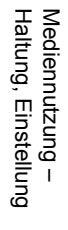 & 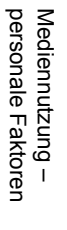 & 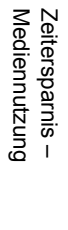 & & & \\
\hline Averbeck, 2014 & & & & & & $\mathrm{x}$ & & \\
\hline Brüggemann, 2014 & & & & & & $x$ & & \\
\hline Drossel, 2018 & & & & $x$ & & & & \\
\hline Eickelmann, 2011 & & & & & & & $x$ & \\
\hline Hankmann, 2014 & $x$ & & & & & $\mathrm{x}$ & & \\
\hline Klemm, 2018 & & $x$ & & & & & & \\
\hline Knaus, 2012 & $x$ & & & & & & & \\
\hline Lorenz \& Endberg, 2016 & & & $x$ & & & & & \\
\hline Ludewig et al., 2013 & $x$ & & & & & $\mathrm{x}$ & & \\
\hline Palkowitsch-Kühl, 2018 & $\mathrm{x}$ & & & & & & & $x$ \\
\hline Schiefner-Rohs, 2014 & & & $x$ & & & & & \\
\hline Schrammel, 2010 & $x$ & $x$ & & & & & & \\
\hline Schulze, 2015 & & $x$ & & & & & & \\
\hline Schweizer \& Horn, 2014 & & & $x$ & $x$ & $x$ & & & \\
\hline Swertz, 2018 & & & & & & & $x$ & \\
\hline Zierer, 2015 & $x$ & & & & & & $x$ & $x$ \\
\hline
\end{tabular}


Bettina Waffner

Tabelle 6: Untersuchungsgegenstand des Analysestranges 3 (74 Studien)

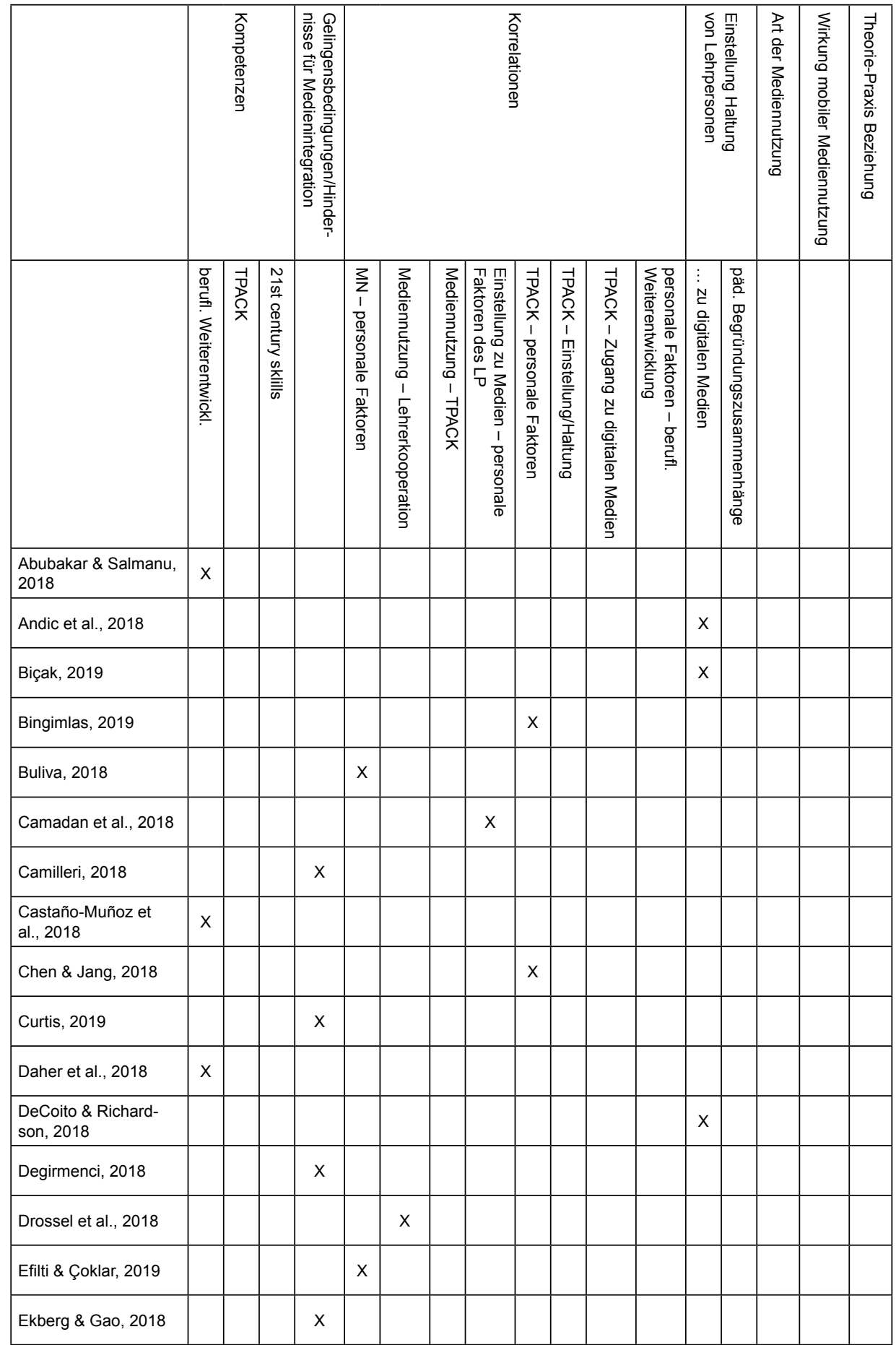


Unterrichtspraktiken, Erfahrungen und Einstellungen |

\begin{tabular}{|c|c|c|c|c|c|c|c|c|c|c|c|c|c|c|c|c|c|}
\hline & & $\begin{array}{l}\text { T. } \\
\stackrel{0}{3} \\
\frac{0}{0} \\
\mathbb{D} \\
\mathbb{1} \\
\mathbb{N} \\
\stackrel{\mathbb{N}}{J}\end{array}$ & & 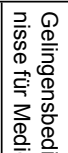 & & & & $\begin{array}{l}7 \\
\frac{\pi}{0} \\
\frac{\pi}{9} \\
\frac{0}{9}\end{array}$ & & & & & & 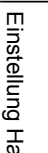 & 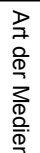 & 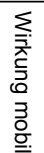 & 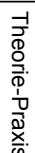 \\
\hline & 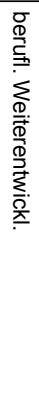 & $\begin{array}{l}-1 \\
\text { गे } \\
\stackrel{\partial}{\lambda}\end{array}$ & 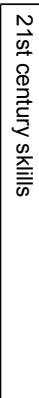 & & 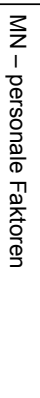 & 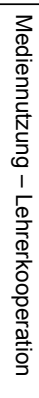 & 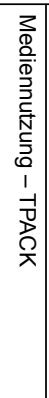 & 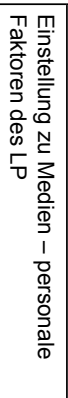 & 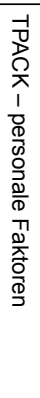 & 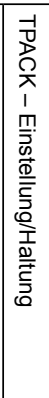 & 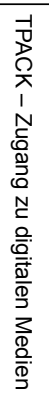 & 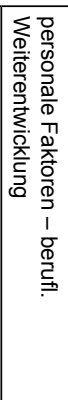 & 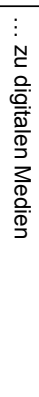 & 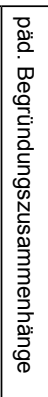 & & & \\
\hline Erduran \& Ince, 2018 & & & & $x$ & & & & & & & & & & & & & \\
\hline $\begin{array}{l}\text { Forkosh-Baruch \& } \\
\text { Hershkovitz, } 2019\end{array}$ & $x$ & & & & & & & & & & & & & & & & \\
\hline Fransson et al., 2019 & & & & & & & & & & & & & $x$ & & & & \\
\hline Frumin et al., 2018 & $x$ & & & & & & & & & & & & & & & & \\
\hline Gregorcic et al., 2018 & & & & $x$ & & & & & & & & & & & & & \\
\hline Herro et al. 2018 & & & & & & & & & & & & & & & $x$ & & \\
\hline Holmberg et al., 2018 & & & & & & & & & & & & & & $x$ & & & \\
\hline $\begin{array}{l}\text { Howlett \& Waemusa, } \\
2018\end{array}$ & & & & & & & & & & & & & $x$ & & & & \\
\hline Islahi \& Nasrin, 2019 & & & & & $\mathrm{x}$ & & & & & & & & & & & & \\
\hline Jong et al., 2018 & & & & & & & & & & & & & $x$ & & & & \\
\hline Kali et al., 2018 & $x$ & & & & & & & & & & & & & & & & \\
\hline Karaseva et al., 2018 & & & & & $x$ & & & & & & & & & & $x$ & & \\
\hline Kaya \& Yazici, 2018 & & & & & & & & & & & & $x$ & & & & & \\
\hline Kearney et al., 2018 & & & & $x$ & $x$ & & & & & & & & & & & & \\
\hline Khlaif, 2018 & & & & $\mathrm{x}$ & & & & & & & & & & & & & \\
\hline Kilinc et al., 2018 & & & & $x$ & & & & & & & & & & & & & \\
\hline Klemm, 2018 & & & $\mathrm{x}$ & & & & & & & & & & & & & & \\
\hline
\end{tabular}




\begin{tabular}{|c|c|c|c|c|c|c|c|c|c|c|c|c|c|c|c|c|c|}
\hline & & 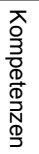 & & 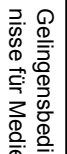 & & & & 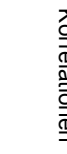 & & & & & 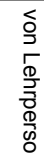 & 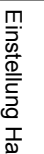 & $\begin{array}{l}\frac{1}{7} \\
\frac{0}{0} \\
\frac{0}{1} \\
3 \\
\frac{1}{0} \\
\frac{2}{0}\end{array}$ & 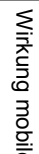 & 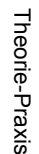 \\
\hline & 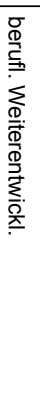 & $\begin{array}{l}\text { ने } \\
\text { गे } \\
\text { م }\end{array}$ & 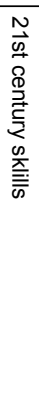 & & 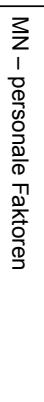 & 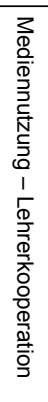 & 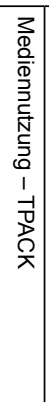 & 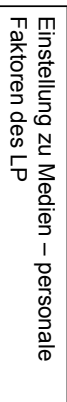 & 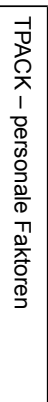 & 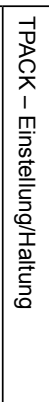 & 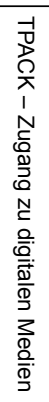 & 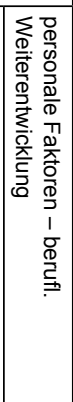 & 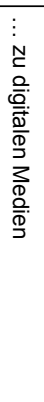 & 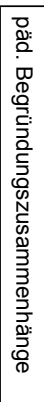 & & & \\
\hline Koh, 2019 & $x$ & & & & & & & & & & & & & & & & \\
\hline $\begin{array}{l}\text { Koukis \& Jimoyiannis, } \\
2019\end{array}$ & $x$ & & & & & & & & & & & & & & & & \\
\hline Kul, 2018 & $\mathrm{x}$ & & & & & & & & & & & & & & & & \\
\hline Lai \& Lin, 2018 & & & & & & & & & & $x$ & & & & & & & \\
\hline Leem \& Sung, 2019 & & & & & & & & & & & & & $x$ & & & & \\
\hline Li et al., 2018a & & & & & & & & & & & & & $x$ & & & & \\
\hline Li et al., 2018b & & & & $x$ & & & & & & & & & & & & & \\
\hline Looi et al., 2018 & $\mathrm{x}$ & & & & & & & & & & & & & & & & \\
\hline Maisamari et al., 2018 & & & & $x$ & & & & & & & & & & & & & \\
\hline $\begin{array}{l}\text { Meletiou-Mavrotheris } \\
\text { et al., } 2019\end{array}$ & $x$ & & & & & & & & & & & & & & & & $x$ \\
\hline Mesfin et al., 2018 & & $x$ & & & & & & & & & $x$ & & & & & & \\
\hline Michos et al., 2018 & & & & & & & & & & & & & & & & & $x$ \\
\hline $\begin{array}{l}\text { Mtebe \& Raphael, } \\
2018\end{array}$ & & $x$ & & & & & & & & & & & & & & & \\
\hline Muhaimin et al., 2019 & & $x$ & & & & & & & & & & & & & & & \\
\hline Muslem et al., 2018 & & & & & & & & & & & & & $x$ & & & & \\
\hline Mwila, 2018 & & & & & & & & & & & & & $x$ & & & & \\
\hline Nami et al., 2018 & & & & & & & & & & & & & & & $x$ & & \\
\hline
\end{tabular}


Unterrichtspraktiken, Erfahrungen und Einstellungen |

\begin{tabular}{|c|c|c|c|c|c|c|c|c|c|c|c|c|c|c|c|c|c|}
\hline & \multicolumn{3}{|c|}{ 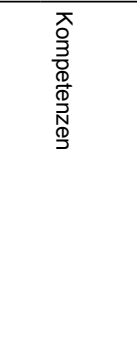 } & \multirow{2}{*}{ 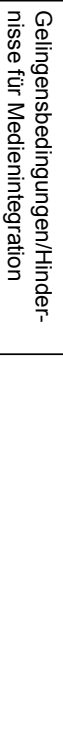 } & \multicolumn{8}{|c|}{ 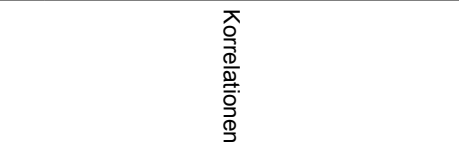 } & \multicolumn{2}{|c|}{ 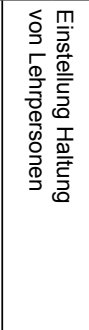 } & \multirow{2}{*}{ 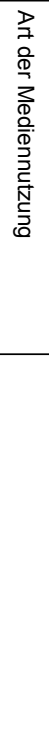 } & \multirow{2}{*}{ 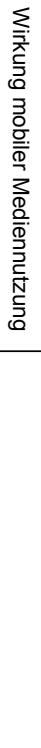 } & \multirow{2}{*}{ 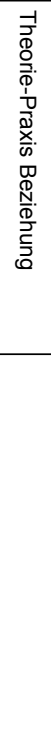 } \\
\hline & 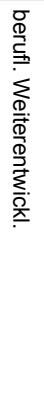 & $\begin{array}{l}-1 \\
\text { गे } \\
\frac{\partial}{\lambda}\end{array}$ & 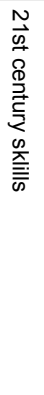 & & 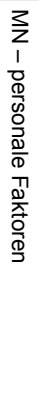 & 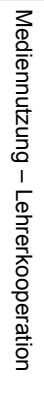 & 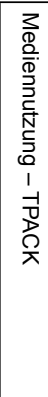 & 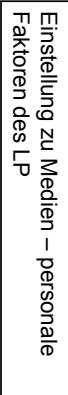 & 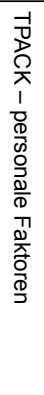 & 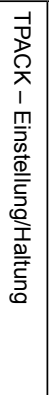 & 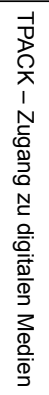 & 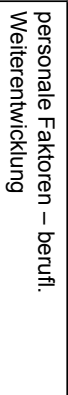 & 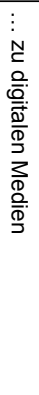 & 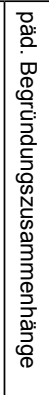 & & & \\
\hline $\begin{array}{l}\text { Ntemngwa \& Oliver, } \\
2018\end{array}$ & & & & $x$ & & & & & & & & & & & & & \\
\hline Nugent et al., 2018 & $x$ & & & & & & & & & & & & & & & & \\
\hline $\begin{array}{l}\text { Palkowitsch-Kühl, } \\
2018\end{array}$ & $x$ & & & & & & & & & & & & & & $x$ & & \\
\hline $\begin{array}{l}\text { Pareja Roblin et al., } \\
2018\end{array}$ & & & & $\mathrm{x}$ & & & & & & & & & & & & & \\
\hline $\begin{array}{l}\text { Pérez-Paredes et al., } \\
2018\end{array}$ & & & & & & & & & & & & & & & $x$ & & \\
\hline Pima, 2019 & & & & $\mathrm{x}$ & & & & & & & & & & & & & \\
\hline Rana et al., 2018 & & & & & & & & & & & & & & & $x$ & & \\
\hline $\begin{array}{l}\text { Reichert \& Mouza, } \\
2018\end{array}$ & & $x$ & & & & & & & & & & & & & & & \\
\hline Rosell-Aguilar, 2018 & $x$ & & & & & & & & & & & & & & & & \\
\hline Sarac, 2018 & & & & & & & & & & & & & $x$ & & & & \\
\hline $\begin{array}{l}\text { Sauers \& McLeod, } \\
2018\end{array}$ & & & & $x$ & & & & & & & & & & & & & \\
\hline $\begin{array}{l}\text { Schuck \& Maher, } \\
2018\end{array}$ & & & & & & & & & & & & & & & & $x$ & \\
\hline $\begin{array}{l}\text { Semerci \& Aydin, } \\
2018\end{array}$ & & & & & & & & & & $x$ & & & & & & & \\
\hline Sheffield et al., 2018 & $x$ & & & & & & & & & & & & & & & & \\
\hline $\begin{array}{l}\text { Simsek \& Sarsar, } \\
2019\end{array}$ & & & & & $x$ & & & & & & & & & & & & \\
\hline Stockless, 2018 & & & & $x$ & & & & & & & & & & & & & \\
\hline $\begin{array}{l}\text { Suárez-Rodríguez et } \\
\text { al., } 2018\end{array}$ & & & & & & & $x$ & & $x$ & & & & & & & & \\
\hline
\end{tabular}


Bettina Waffner

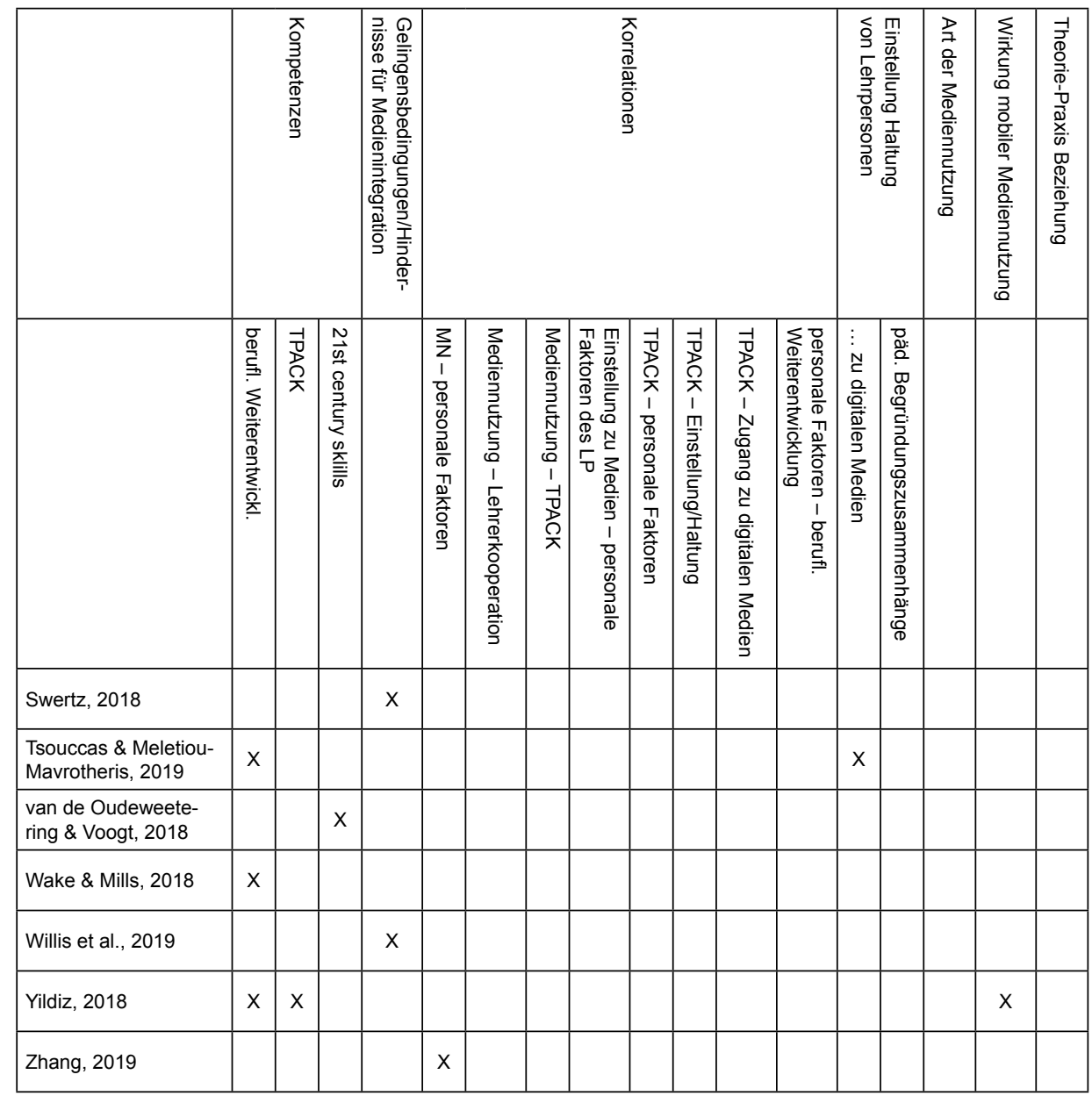




\section{Literatur}

* durch Reviewprozess ausgewählte Studie

*Abubakar, H. \& Salmanu, Y. (2018). Impact of Internet Technology Usage on Job Performance of Senior Secondary School Teachers in Kaduna State Nigeria. International Journal of Curriculum and Instruction, 10(2), 152-167.

*Albion, P. R., Tondeur, J., Forkosh-Baruch, A. \& Peeraer, J. (2015). Teachers' professional development for ICT integration: Towards a reciprocal relationship between research and practice. Education and Information Technologies, 20(4), 655-673. https:// doi.org/10.1007/s10639-015-9401-9

${ }^{\star}$ Al-Zaidiyeen, N. J., Mei, L. L. \& Fook, F. S. (2010). Teachers' Attitudes and Levels of Technology Use in Classrooms: The Case of Jordan Schools. International Education Studies, 3(2), 211-218. https://eric.ed.gov/?id=EJ1066020. https://doi.org/10.5539/ies. v3n2p211

*Andic, B., Kadic, S., Grujicic, R., Malidžan, D. (2018). A Comparative Analysis of the Attitudes of Primary School Students and Teachers Regarding the Use of Games in Teaching. IAFOR Journal of Education, 6(2), 5-16. https://eric.ed.gov/?id=EJ1181054. https://doi.org/10.22492/ije.6.2.01

Anfara, V. A., Brown, K. M. \& Mangione, T. L. (2002). Qualitative Analysis on Stage: Making the Research Process More Public. Educational Researcher, 31(7), 28-38. https://doi.org/10.3102/0013189X031007028

*Anthony, A. B. (2012). Activity Theory as a Framework for Investigating DistrictClassroom System Interactions and Their Influences on Technology Integration. Journal of Research on Technology in Education, 44(4), 335-356. https://eric.ed.gov/?id= EJ976472. https://doi.org/10.1080/15391523.2012.10782594

*Averbeck, I. \& Welling, S. (2014). Medienkompetenzförderung am Übergang von der Primarstufe in die weiterführenden Schulen. Medien + Erziehung, 58(6), 28-40.

${ }^{\star}$ Biçak, F. (2019). Investigation of the Views of Teachers toward the Use of Smart Boards in the Teaching and Learning Process, 3(1), 15-23. https://eric.ed.gov/?id=ED594103. https://doi.org/10.33902/JPR.2019.1

${ }^{\star}$ Bingimlas, K. (2018). Investigating the Level of Teachers' Knowledge in Technology, Pedagogy, and Content (TPACK) in Saudi Arabia. South African Journal of Education, 38(3), Artikel 1496. https://eric.ed.gov/?id=EJ1191284. https://doi.org/10.15700/saje. v38n3a1496

BMBF \& KMK. (2019). Schule macht stark: Gemeinsame Initiative von Bund und Ländern zur Unterstützung von Schulen in sozial schwierigen Lagen. https://www.bmbf.de/files/ Schule\%20macht\%20stark_Bund-Länder-Vereinbarung.pdf

Bos, W. (Hrsg.). (2014). ICILS 2013: Computer- und informationsbezogene Kompetenzen von Schülerinnen und Schülern in der 8. Jahrgangsstufe im internationalen Vergleich. Münster: Waxmann.

*Brüggemann, M. (2014). Medienpädagogische Orientierungsmuster berufserfahrener Lehrkräfte. Medien + Erziehung, 58(6), 63-73.

${ }^{\star}$ Buliva, N. (2018). Teachers' Attitudes towards the Utility of Computers in Education in Kenya. African Educational Research Journal, 6(1), 5-9. https://eric.ed.gov/?id= EJ1208525. https://doi.org/10.30918/AERJ.61.18.004

${ }^{\star}$ Camadan, F., Reisoglu, I., Ursavas, Ö. F. \& Mcilroy, D. (2018). How Teachers' Personality Affect on Their Behavioral Intention to Use Tablet PC. International Journal of Information and Learning Technology, 35(1), 12-28. 
${ }^{*}$ Camilleri, P. (2018). Looking for Cracks in the Pavement: Maltese Teachers' Temporal Adaptation Patterns toward Tablet PC Use in Formal Educational Settings. Contemporary Issues in Technology and Teacher Education (CITE Journal), 18(2). http:// www.citejournal.org/volume-18/issue-2-18/current-practice/looking-for-cracks-in-thepavement-maltese-teachers-temporal-adaptation-patterns-toward-tablet-pc-use-in-for mal-educational-settings/

${ }^{\star}$ Carpenter, J. P. \& Krutka, D. G. (2014). How and Why Educators Use Twitter: A Survey of the Field. Journal of Research on Technology in Education, 46(4), 414-434. https://doi.org/ 10.1080/15391523.2014.925701

${ }^{\star}$ Carpenter, J. P. \& Krutka, D. G. (2015). Engagement through Microblogging: Educator Professional Development via Twitter. Professional Development in Education, 41(4), 707-728. https://doi.org/10.1080/19415257.2014.939294

${ }^{*}$ Cassidy, W., Brown, K. \& Jackson, M. (2012). "Under the Radar": Educators and Cyberbullying in Schools. School Psychology International, 33(5), 520-532. https://doi. org/10.1177/0143034312445245

${ }^{\star}$ Castaño-Muñoz, J., Kalz, M., Kreijns, K. \& Punie, Y. (2018). Who Is Taking MOOCs for Teachers' Professional Development on the Use of ICT? A Cross-Sectional Study from Spain. Technology, Pedagogy and Education, 27(5), 607-624. https://doi.org/10.1080/14 75939X.2018.1528997

${ }^{*}$ Chang, I.-H. (2012). The Effect of Principals' Technological Leadership on Teachers' Technological Literacy and Teaching Effectiveness in Taiwanese Elementary Schools. Educational Technology \& Society, 15(2), 328-340. http://www.ifets.info/journals/15_2/ 28.pdf

${ }^{\star}$ Chen, Y.-H. \& Jang, S.-J. (2018). Exploring the Relationship Between Self-Regulation and TPACK of Taiwanese Secondary In-Service Teachers. Journal of Educational Computing Research, 57(4), 978-1002. https://doi.org/10.1177/0735633118769442

${ }^{*}$ Chiu, T. K. F. \& Churchill, D. (2016). Adoption of Mobile Devices in Teaching: Changes in Teacher Beliefs, Attitudes and Anxiety. Interactive Learning Environments, 24(2), 317327. https://doi.org/10.1080/10494820.2015.1113709

${ }^{\star}$ Ciampa, K. (2014). Learning in a Mobile Age: An Investigation of Student Motivation. Journal of Computer Assisted Learning, 30(1), 82-96. https://doi.org/10.1111/jcal.12036

${ }^{\star}$ Curtis, M. D. (2019). Professional Technologies in Schools: The Role of Pedagogical Knowledge in Teaching with Geospatial Technologies. Journal of Geography, 118(3), 130-142. https://doi.org/10.1080/00221341.2018.1544267

${ }^{\star}$ Daher, W., Baya'a, N. \& Anabousy, R. (2018). In-Service Mathematics Teachers' Integration of ICT as Innovative Practice. International Journal of Research in Education and Science, 4(2), 534-543. https://eric.ed.gov/?id=EJ1185069. https://doi.org/10.21890/ ijres. 428945

${ }^{\star}$ DeCoito, I. \& Richardson, T. (2018). Teachers and Technology: Present Practice and Future Directions. Contemporary Issues in Technology and Teacher Education (CITE Journal), 18(2). http://www.citejournal.org/volume-18/issue-2-18/science/teachers-and-technolo gy-present-practice-and-future-directions/

${ }^{*}$ Degirmenci, Y. (2018). Use of Geographic Information Systems (GIS) in Geography Lessons According to Teachers' Opinion. World Journal on Educational Technology: Current Issues, 10(3), 186-196. https://doi.org/10.18844/wjet.v10i3.3559

${ }^{*}$ Drossel, K., Eickelmann, B. \& Gerick, J. (2017). Predictors of Teachers' Use of ICT in School - The Relevance of School Characteristics, Teachers' Attitudes and Teacher Collaboration. Education and Information Technologies, 22(2), 551-573. https://doi.org/ 10.1007/s10639-016-9476-y 
${ }^{*}$ Drossel, K., Eickelmann, B. \& Lorenz, R. (2018). Determinanten der unterrichtlichen Computernutzungshäufigkeit und der medienbezogenen Kooperation. Unterrichtswissenschaft, 46(4), 481-498. https://doi.org/10.1007/s42010-018-0017-9

*Efilti, E. \& Çoklar, A. N. (2019). Teachers' Technostress Levels as an Indicator of Their Psychological Capital Levels. Universal Journal of Educational Research, 7(2), 413-421. https://doi.org/10.13189/ujer.2019.070214

*Eickelmann, B. (2011). Supportive and hindering factors to a sustainable implementation of ICT in schools: Paralleltitel: Förderliche und hemmende Bedingungen der nachhaltigen Implementierung von IKT in Schulen. Journal for educational research online, 3(1), 75-103. http://nbn-resolving.de/urn:nbn:de:0111-opus-46836

${ }^{*}$ Ekberg, S. \& Gao, S. (2018). Understanding Challenges of Using ICT in Secondary Schools in Sweden from Teachers' Perspective. International Journal of Information and Learning Technology, 35(1), 43-55. https://doi.org/10.1108/IJILT-01-2017-0007

*Erduran, A. \& Ince, B. (2018). Identifying Mathematics Teachers' Difficulties in Technology Integration in Terms of Technological Pedagogical Content Knowledge (TPCK). International Journal of Research in Education and Science, 4(2), 555-576. https://doi. org/10.21890/ijres.428955

*Forkosh-Baruch, A. \& Hershkovitz, A. (2019). Knowing Me, Knowing You: Teachers' Perceptions of Communication with Their Students on Facebook. Interdisciplinary Journal of e-Skills and Lifelong Learning, 15, 59-80. https://doi.org/10.28945/4256

*Fransson, G., Holmberg, J., Lindberg, O. J. \& Olofsson, A. D. (2019). Digitalise and Capitalise? Teachers' Self-Understanding in 21st-Century Teaching Contexts. Oxford Review of Education, 45(1), 102-118. https://doi.org/10.1080/03054985.2018.1500357

${ }^{\star}$ Frumin, K., Dede, C., Fischer, C., Foster, B., Lawrenz, F., Eisenkraft, A., Fishman, B., Jurist Levy, A. \& McCoy, A. (2018). Adapting to Large-Scale Changes in Advanced Placement Biology, Chemistry, and Physics: The Impact of Online Teacher Communities. International Journal of Science Education, 40(4), 397-420. https://doi. org/10.1080/09500693.2018.1424962

Fullan, M. G., Stiegelbauer, S. M. \& Fullan, M. (1991). The new meaning of educational change (2. Auflage). Teachers College Press Teachers College Columbia Univ.; Ontario Institute for Studies in Education.

${ }^{*}$ Gil-Flores, J., Rodríguez-Santero, J. \& Torres-Gordillo, J.-J. (2017). Factors that explain the use of ICT in secondary-education classrooms: The role of teacher characteristics and school infrastructure. Computers in Human Behavior, 68, 441-449. https://doi. org/10.1016/j.chb.2016.11.057

${ }^{\star}$ Gregorcic, B., Etkina, E. \& Planinsic, G. (2018). A New Way of Using the Interactive Whiteboard in a High School Physics Classroom: A Case Study. Research in Science Education, 48(2), 465-489. https://doi.org/10.1007/s11165-016-9576-0

${ }^{\star}$ Hankmann, M. (2014). Technik-Muffel oder digitale Avantgarde? Zur Ausstattung und Nutzung digitaler Medien in Schulen. Bildung spezial, 4(4), 20-22.

Hattie, J. (2009). Visible learning: A synthesis of over 800 meta-analyses relating to achievement. Routledge. https://doi.org/10.4324/9780203887332

${ }^{\star}$ Henriksen, D., Mishra, P. \& Fisser, P. (2016). Infusing Creativity and Technology in 21st Century Education: A Systemic View for Change. Educational Technology \& Society, 19(3), 27-37.

${ }^{\star}$ Herro, D., Quigley, C. \& Jacques, L. A. (2018). Examining Technology Integration in Middle School STEAM Units. Technology, Pedagogy and Education, 27(4), 485-498. https://doi.org/10.1080/1475939X.2018.1514322 
${ }^{\star}$ Holmberg, J., Fransson, G. \& Fors, U. (2018). Teachers' Pedagogical Reasoning and Reframing of Practice in Digital Contexts. International Journal of Information and Learning Technology, 35(2), 130-142. https://doi.org/10.1108/IJILT-09-2017-0084

${ }^{\star}$ Howard, S. K. (2013). Risk-Aversion: Understanding Teachers' Resistance to Technology Integration. Technology, Pedagogy and Education, 22(3), 357-372. https://doi.org/10.10 80/1475939X.2013.802995. https://doi.org/10.30935/cet.471007

*Howlett, G. \& Waemusa, Z. (2018). Digital Native/Digital Immigrant Divide: EFL Teachers' Mobile Device Experiences and Practice. Contemporary Educational Technology, 9(4), 374-389. https://eric.ed.gov/?id=EJ1194247

${ }^{\star}$ Howley, A., Wood, L. \& Hough, B. (2011). Rural Elementary School Teachers' Technology Integration. Journal of Research in Rural Education, 26(9). http://jrre.psu.edu/arti cles/26-9.pdf

${ }^{\star}$ Hsu, P.-S. (2016). Examining Current Beliefs, Practices and Barriers About Technology Integration: A Case Study. TechTrends, 60. https://doi.org/10.1007/s11528-015-0014-3

${ }^{*}$ Islahi, F. \& Nasrin (2019). Exploring Teacher Attitude towards Information Technology with a Gender Perspective. Contemporary Educational Technology, 10(1), 37-54. https://doi.org/10.30935/cet.512527

${ }^{*}$ Jong, D. de, Grundmeyer, T. \& Anderson, C. (2018). Comparative Study of Elementary and Secondary Teacher Perceptions of Mobile Technology in Classrooms. International Journal of Mobile and Blended Learning, 10(1), 12-33, Artikel 2. https://doi. org/10.4018/IJMBL.2018010102

${ }^{\star}$ Kale, U. \& Goh, D. (2014). Teaching Style, ICT Experience and Teachers' Attitudes toward Teaching with Web 2.0. Education and Information Technologies, 19(1), 41-60. https:// doi.org/10.1007/s10639-012-9210-3

${ }^{\star}$ Kali, Y., Levy, K.-S., Levin-Peled, R. \& Tal, T. (2018). Supporting Outdoor Inquiry Learning (SOIL): Teachers as Designers of Mobile-Assisted Seamless Learning. British Journal of Educational Technology, 49(6), 1145-1161. https://doi.org/10.1111/bjet.12698

${ }^{\star}$ Karaseva, A., Pruulmann-Vengerfeldt, P. \& Siibak, A. (2018). Relationships between InService Teacher Achievement Motivation and Use of Educational Technology: Case Study with Latvian and Estonian Teachers. Technology, Pedagogy and Education, 27(1), 33-47. https://doi.org/10.1080/1475939X.2017.1339633

${ }^{\star}$ Kaya, M. T. \& Yazici, H. (2018). Self-Efficacy of the Social Studies Teachers in Using the Interactive Whiteboards. Review of International Geographical Education Online, 8(3), 601-612. https://doi.org/10.33403/rigeo.505285

^Kearney, M., Schuck, S., Aubusson, P. \& Burke, P. F. (2018). Teachers' Technology Adoption and Practices: Lessons Learned from the IWB Phenomenon. Teacher Development, 22(4), 481-496. https://doi.org/10.1080/13664530.2017.1363083

${ }^{\star}$ Kereluik, K., Mishra, P., Fahnoe, C. \& Terry, L. (2013). What Knowledge Is of Most Worth: Teacher Knowledge for 21st Century Learning. Journal of Digital Learning in Teacher Education, 29(4), 127-140. https://eric.ed.gov/?id=EJ1010753. https://doi.org/10.1080/2 1532974.2013 .10784716

Kerres, M. (2020). Bildung in der digitalen Welt: Über Wirkungsannahmen und die soziale Konstruktion des Digitalen. MedienPädagogik. Zeitschrift für Theorie und Praxis der Medienbildung. [preprint]. https://www.medienpaed.com/. https://doi.org/10.21240/ $\mathrm{mpaed} / \mathrm{jb} 17 / 2020.04 .24 . \mathrm{X}$

Kerres, M. \& Waffner, B. (2019). Digital School Networks: Technology Integration as a Joint Research and Development Effort. In R. M. Reardon \& J. Leonard (Hrsg.), Integrating Digital Technology in Education: School-University-Community Collaboration (S. 227241). Charlotte. 
${ }^{\star}$ Khlaif, Z. (2018). Teachers' Perceptions of Factors Affecting Their Adoption and Acceptance of Mobile Technology in K-12 Settings. Computers in the Schools, 35(1), 49-67. https://doi.org/10.1080/07380569.2018.1428001

${ }^{\star}$ Kilinc, E., Tarman, B. \& Aydin, H. (2018). Examining Turkish Social Studies Teachers' Beliefs about Barriers to Technology Integration. TechTrends: Linking Research and Practice to Improve Learning, 62(3), 221-223. https://doi.org/10.1007/s11528-018$0280-y$

${ }^{\star}$ Klemm, M. (2018). Digitale Kompetenz. Pädagogische Rundschau, 72(6), 603-722.

${ }^{\star}$ Knaus, T. (2012). Was Lehrerinnen und Lehrer lernen (wollen). Basics schulischer Medienbildung. Computer + Unterricht, 22(87), 11-13.

${ }^{\star}$ Koh, J. H. L. (2019). TPACK design scaffolds for supporting teacher pedagogical change. Educational Technology Research and Development, 67(3), 577-595. https://doi.org/10. 1007/s11423-018-9627-5

${ }^{\star}$ Kopcha, T. J. (2012). Teachers' Perceptions of the Barriers to Technology Integration and Practices with Technology under Situated Professional Development. Computers \& Education, 59(4), 1109-1121. https://doi.org/10.1016/j.compedu.2012.05.014

${ }^{\star}$ Koukis, N. \& Jimoyiannis, A. (2019). MOOCs for Teacher Professional Development: Exploring Teachers' Perceptions and Achievements. Interactive Technology and Smart Education, 16(1), 74-91. https://doi.org/10.1108/ITSE-10-2018-0081

${ }^{\star}$ Kul, U. (2018). Influences of Technology Integrated Professional Development Course on Mathematics Teachers. European Journal of Educational Research, 7(2), 233-243. https://doi.org/10.12973/eu-jer.7.2.233

${ }^{\star}$ Lai, T.-l. \& Lin, H.-F. (2018). An Investigation of the Relationship of Beliefs, Values and Technological Pedagogical Content Knowledge among Teachers. Technology, Pedagogy and Education, 27(4), 445-458. https://doi.org/10.1080/1475939X.2018.1496137

${ }^{\star}$ Lee, J., Cerreto, F. A. \& Lee, J. (2010). Theory of Planned Behavior and Teachers' Decisions regarding Use of Educational Technology. Educational Technology \& Society, 13(1), 152-164. http://www.ifets.info/journals/13_1/15.pdf

${ }^{\star}$ Leem, J. \& Sung, E. (2019). Teachers' Beliefs and Technology Acceptance Concerning SMART Mobile Devices for Smart Education in South Korea. British Journal of Educational Technology, 50(2), 601-613. https://doi.org/10.1111/bjet.12612

${ }^{\star}$ Li, S., Yamaguchi, S. \& Takada, J.-I. (2018). Understanding Factors Affecting Primary School Teachers' Use of ICT for Student-Centered Education in Mongolia. International Journal of Education and Development using Information and Communication Technology, 14(1), 103-117. https://eric.ed.gov/?id=EJ1178363

${ }^{\star}$ Li, Y., Garza, V., Keicher, A. \& Popov, V. (2018). Predicting High School Teacher Use of Technology: Pedagogical Beliefs, Technological Beliefs and Attitudes, and Teacher Training. Technology, Knowledge and Learning. Advance online publication. https://doi. org/10.1007/s10758-018-9355-2

${ }^{*}$ Lin, T.-C., Tsai, C.-C., Chai, C. S. \& Lee, M.-H. (2013). Identifying Science Teachers' Perceptions of Technological Pedagogical and Content Knowledge (TPACK). Journal of Science Education and Technology, 22(3), 325-336. https://doi.org/10.1007/s10956012-9396-6

${ }^{\star}$ Liu, S.-H. (2011). Factors Related to Pedagogical Beliefs of Teachers and Technology Integration. Computers \& Education, 56(4), 1012-1022. https://doi.org/10.1016/j. compedu.2010.12.001

${ }^{*}$ Looi, C.-K., Sun, D., Kim, M. S. \& Wen, Y. (2018). The Impact of a Professional Development Model for a Mobilized Science Curriculum: A Case Study of Teacher Changes. Research in Science \& Technological Education, 36(1), 86-110. https://doi.org/10.1080/0 2635143.2017.1409704 
${ }^{*}$ Lorenz, R. \& Endberg, M. (2016). Zusammenhang zwischen medienbezogenen Lehrereinstellungen und der Förderung computer- und informationsbezogener Kompetenzen. Jahrbuch der Schulentwicklung, (19), 206-229. https://www.beltz.de/fachmedien/pae dagogik/buecher/produkt_produktdetails/33471-jahrbuch_der_schulentwicklung_ band_19.html

${ }^{\star}$ Ludewig, Y., Knaus, T. \& Döring, N. (2013). Die Wirksamkeit von Medienbildungsinitiativen: Erfolge, Probleme und Lösungsansätze. Eine Einschätzung der Schulleitungen und Lehrkräfte allgemeinbildender Schulen der Stadt Frankfurt am Main. MedienPädagogik (06.09.2013), 19 S. http://nbn-resolving.de/urn:nbn:de:0111-pedocs117025; http://www.medienpaed.com/globalassets/medienpaed/2013/ludewig1309.pdf. https://doi.org/10.21240/mpaed/00/2013.09.06.X

${ }^{\star}$ Lynch, J. \& Redpath, T. (2014). "Smart" Technologies in Early Years Literacy Education: A Meta-Narrative of Paradigmatic Tensions in iPad Use in an Australian Preparatory Classroom. Journal of Early Childhood Literacy, 14(2), 147-174. https://doi.org/ $10.1177 / 1468798412453150$

${ }^{\star}$ Maisamari, A. M., Adikwu, V. O., Ogwuche, C. O. \& Ikwoche, F. I. (2018). Assessment of Secondary School Teachers' Use of Information and Communication Technology (ICT) in Anyingba Metropolis, Kogi State, Nigeria, 5(1), 32-47. https://eric.ed.gov/ ?id=ED583753

Martín-Martín, A., Orduna-Malea, E., Thelwall, M. \& López-Cózar, E. D. (2018). Google Scholar, Web of Science, and Scopus: a systematic comparison of citations in 252 subject categories. https://doi.org/10.31235/osf.io/42nkm

${ }^{\star}$ Meletiou-Mavrotheris, M., Paparistodemou, E. \& Christou, C. M. (2019). Integrating Mobile Devices in the Mathematics Curriculum: A Case Study of a Primary School in Cyprus. International Journal of Mobile and Blended Learning, 11(3), 19-37. https://doi. org/10.4018/IJMBL.2019070102

*Mesfin, G., Ghinea, G., Grønli, T.-M. \& Hwang, W.-Y. (2018). Enhanced Agility of E-Learning Adoption in High Schools. Educational Technology \& Society, 21(4), 157170. https://www.j-ets.net/ETS/journals/21_4/14.pdf

${ }^{\star}$ Michos, K., Hernández-Leo, D. \& Albó, L. (2018). Teacher-Led Inquiry in TechnologySupported School Communities. British Journal of Educational Technology, 49(6), 1077-1095. https://doi.org/10.1111/bjet.12696

Ministerium für Schule und Weiterbildung des Landes Nordrhein-Westfalen. (2014). Rahmenvorgaben für den Schulsport in Nordrhein-Westfalen: Stufenübergreifende Unterrichtsvorgaben - alle Schulstufen; Richtlinien und Lehrpläne; Rahmenvorgaben für den Schulsport. https://www.schulentwicklung.nrw.de/lehrplaene/upload/klp_SI/HS/sp/ Rahmenvorgaben_Schulsport_Endfassung.pdf

Mishra, P. \& Koehler, M. (2006). Technological Pedagogical Content Knowledge: A Framework for Teacher Knowledge. Teachers College Record, 108, 1017-1054. https:// doi.org/10.1111/j.1467-9620.2006.00684.x

*Mtebe, J. S. \& Raphael, C. (2018). Eliciting In-Service Teachers' Technological Pedagogical Content Knowledge for 21st-Century Skills in Tanzania. Journal of Learning for Development, 5(3), 263-279.

${ }^{\star}$ Muhaimin, M., Habibi, A., Mukminin, A., Saudagar, F., Pratama, R., Wahyuni, S., Sadikin, A. \& Indrayana, B. (2019). A Sequential Explanatory Investigation of TPACK: Indonesian Science Teachers' Survey and Perspective. Journal of Technology and Science Education, 9(3), 269-281. https://doi.org/10.3926/jotse.662

${ }^{*}$ Muslem, A., Yusuf, Y. Q. \& Juliana, R. (2018). Perceptions and Barriers to ICT Use among English Teachers in Indonesia. Teaching English with Technology, 18(1), 3-23. 
${ }^{*}$ Mwila, P. (2018). Assessing the Attitudes of Secondary School Teachers towards the Integration of ICT in the Teaching Process in Kilimanjaro, Tanzania. International Journal of Education and Development using Information and Communication Technology, 14(3), 223-238.

${ }^{*}$ Nami, F., Marandi, S. S. \& Sotoudehnama, E. (2018). Interaction in a Discussion List: An Exploration of Cognitive, Social, and Teaching Presence in Teachers' Online Collaborations. ReCALL, 30(2), 375-398. https://doi.org/10.1017/S0958344017000349

${ }^{\star}$ Niess, M. L. (2011). Investigating TPACK: Knowledge Growth in Teaching with Technology. Journal of Educational Computing Research, 44(3), 299-317. https://doi. org/10.2190/EC.44.3.c

${ }^{*}$ Ntemngwa, C. \& Oliver, J. S. (2018). The Implementation of Integrated Science Technology, Engineering and Mathematics (STEM) Instruction Using Robotics in the Middle School Science Classroom. International Journal of Education in Mathematics, Science and Technology, 6(1), 12-40. https://eric.ed.gov/?id=EJ1168684. https://doi. org/10.18404/ijemst.380617

${ }^{\star}$ Nugent, G., Kunz, G., Houston, J., Wu, C., Patwardhan, I., Lee, S., DeChenne-Peters, S. E. \& Luo, L. (2018). The Effectiveness of a Summer Institute and Remotely Delivered Science Instructional Coaching in Middle and High School. Journal of Science Teacher Education, 29(8), 760-784. https://doi.org/10.1080/1046560X.2018.1514193

Oelkers, J. (2016). Lehrer oder Lerncoach? Über die Zukunft des Unterrichtens. New York Review of Books, 63(11), 18 S.

${ }^{\star}$ Olofson, M. W., Swallow, M. J. C. \& Neumann, M. D. (2016). TPACKing: A constructivist framing of TPACK to analyze teachers' construction of knowledge. Computers \& Education, 95, 188-201. https://doi.org/10.1016/j.compedu.2015.12.010

${ }^{\star}$ Ozdamli, F. \& Uzunboylu, H. (2015). M-Learning Adequacy and Perceptions of Students and Teachers in Secondary Schools. British Journal of Educational Technology, 46(1), 159-172. https://doi.org/10.1111/bjet.12136

*Palkowitsch-Kühl, J. (2018). Digitale Medienkompetenz - eine Schlüsselkompetenz in der Lehrkräfteausbildung. Aktuelle Perspektiven von Lehrkräften im Bereich schulisch verantworteter religiöser Bildung. Zeitschrift für Pädagogik und Theologie, 70(3), 294-307. https://doi.org/10.1515/zpt-2018-0035

*Pareja Roblin, N., Tondeur, J., Voogt, J., Bruggeman, B., Mathieu, G. \& van Braak, J. (2018). Practical Considerations Informing Teachers' Technology Integration Decisions: The Case of Tablet PCs. Technology, Pedagogy and Education, 27(2), 165-181. https://doi.org/ 10.1080/1475939X.2017.1414714

*Pérez-Paredes, P., Ordoñana Guillamón, C. \& Aguado Jiménez, P. (2018). Language Teachers' Perceptions on the Use of OER Language Processing Technologies in MALL. Computer Assisted Language Learning, 31, 522-545. https://doi.org/10.1080/09588221. 2017.1418754

*Perrotta, C. (2013). Do School-Level Factors Influence the Educational Benefits of Digital Technology? A Critical Analysis of Teachers' Perceptions. British Journal of Educational Technology, 44(2), 314-327. https://doi.org/10.1111/j.1467-8535.2012.01304.x

*Petko, D. (2012). Teachers' Pedagogical Beliefs and Their Use of Digital Media in Classrooms: Sharpening the Focus of the "Will, Skill, Tool" Model and Integrating Teachers' Constructivist Orientations. Computers \& Education, 58(4), 1351-1359. https://doi.org/10.1016/j.compedu.2011.12.013

*Pima, J. M. (2019). Factors That Motivate Teachers to Use ICT in Teaching: A Case of Kaliua District Secondary Schools in Tanzania. International Journal of Education and Development using Information and Communication Technology, 15(1). 
${ }^{\star}$ Quek, C. L. \& Wang, Q. (2014). Exploring Teachers' Perceptions of Wikis for Learning Classroom Cases. Australian Journal of Teacher Education, 39(2), Artikel 8. https://eric. ed.gov/?id=EJ1016508. https://doi.org/10.14221/ajte.2014v39n2.2

${ }^{\star}$ Rana, K., Greenwood, J., Fox-Turnbull, W. \& Wise, S. (2018). A Shift from Traditional Pedagogy in Nepali Rural Primary Schools? Rural Teachers' Capacity to Reflect ICT Policy in Their Practice. International Journal of Education and Development using Information and Communication Technology, 14(3), 149-166.

${ }^{*}$ Reichert, M. \& Mouza, C. (2018). Teacher Practices during Year 4 of a One-to-One Mobile Learning Initiative. Journal of Computer Assisted Learning, 34(6), 762-774. https://doi. org/10.1111/jcal.12283

Rolff, H.-G. (2011). Wie verändern wir Schule wirklich? Gelingensbedingungen für erfolgreiche Schulentwicklung. In V. Lange (Hrsg.), Schriftenreihe des Netzwerk Bildung: $B d$. 22. Schulentwicklung zwischen Autonomie und Kontrolle: Wie verändern wir Schule wirklich? (S. 23-32). Friedrich-Ebert-Stiftung.

Rolff, H.-G. (2016). Schulentwicklung kompakt: Modelle, Instrumente, Perspektiven (3. Auflage). Pädagogik. Beltz.

${ }^{\star}$ Rosell-Aguilar, F. (2018). Twitter: A Professional Development and Community of Practice Tool for Teachers. Journal of Interactive Media in Education, (1). https://doi. org/10.5334/jime.452

${ }^{\star}$ Ruggiero, D. \& Mong, C. J. (2015). The Teacher Technology Integration Experience: Practice and Reflection in the Classroom. Journal of Information Technology Education: Research, 14, 161-178. http://www.jite.org/documents/Vol14/JITEv14ResearchP161178Ruggiero0958.pdf

*Sarac, H. (2018). Use of Instructional Technologies by Teachers in the Educational Process: Metaphor Analysis Study. European Journal of Educational Research, 7(2), 189-202. https://eric.ed.gov/?id=EJ1175344. https://doi.org/10.12973/eu-jer.7.2.189

*Sauers, N. J. \& McLeod, S. (2018). Teachers' Technology Competency and Technology Integration in 1:1 Schools. Journal of Educational Computing Research, 56(6), 892-910. https://doi.org/10.1177/0735633117713021

${ }^{\star}$ Schiefner-Rohs, M. (2014). Grenzenlose Medienbildung in der Schule? (Pädagogisches) Medienhandeln unter Bedingungen der Bildungsinstitution Schule. Medien + Erziehung, 58(6), 74-82.

${ }^{\star}$ Schrammel, S. (2010). Medienpädagogisch professionelles LehrerInnenhandeln. MedienImpulse (3), $14 \mathrm{~S}$. http://www.medienimpulse.at/articles/view/250

${ }^{*}$ Schuck, S. \& Maher, D. (2018). Creating Opportunities for Untethered Learning. Technology, Pedagogy and Education, 27(4), 473-484. https://doi.org/10.1080/147593 9X.2018.1510788

${ }^{*}$ Schulze, U., Gryl, I. \& Kanwischer, D. (2015). Spatial Citizenship - Zur Entwicklung eines Kompetenzstrukturmodells für eine fächerübergreifende Lehrerfortbildung. Zeitschrift für Geographiedidaktik, 43(2), 139-164.

${ }^{\star}$ Schweizer, K. \& Horn, M. (2014). Kommt es auf die Einstellung zu digitalen Medien an? Normative Überzeugungen, personale Faktoren und digitale Medien im Unterricht: Eine Untersuchung mit Lehrpersonen und Lehramtsstudierenden. Medien + Erziehung, 58(6), 50-62.

*Semerci, A. \& Aydin, M. K. (2018). Examining High School Teachers' Attitudes towards ICT Use in Education. International Journal of Progressive Education, 14(2), 93-105. https://eric.ed.gov/?id=EJ1177301. https://doi.org/10.29329/ijpe.2018.139.7

*Sheffield, R., Blackley, S. \& Moro, P. (2018). A Professional Learning Model Supporting Teachers to Integrate Digital Technologies. Issues in Educational Research, 28(2), 487510. http://www.iier.org.au/iier28/sheffield.pdf 
*Simsek, Ö. \& Sarsar, F. (2019). Investigation of the Self-Efficacy of the Teachers in Technological Pedagogical Content Knowledge and Their Use of Information and Communication Technologies. World Journal of Education, 9(1), 196-208. https://eric. ed.gov/?id=EJ1208813. https://doi.org/10.5430/wje.v9n1p196

*Sipilä, K. (2014). Educational Use of Information and Communications Technology: Teachers' Perspective. Technology, Pedagogy and Education, 23(2), 225-241. https://doi. org/10.1080/1475939X.2013.813407

Soffel, J. (2016). What are the 21st-century skills every student needs? World Economic Forum. https://www.weforum.org/agenda/2016/03/21st-century-skills-future-jobs-stu dents/

*Starkey, L. (2011). Evaluating Learning in the 21st Century: A Digital Age Learning Matrix. Technology, Pedagogy and Education, 20(1), 19-39. https://doi.org/10.1080/14 75939X.2011.554021

${ }^{\star}$ Stockless, A. (2018). Acceptance of Learning Management System: The Case of Secondary School Teachers. Education and Information Technologies, 23(3), 1101-1121. https:// doi.org/10.1007/s10639-017-9654-6

*Suárez-Rodríguez, J., Almerich, G., Orellana, N. \& Díaz-García, I. (2018). A Basic Model of Integration of ICT by Teachers: Competence and Use. Educational Technology Research and Development, 66(5), 1165-1187. https://doi.org/10.1007/s11423-018-9591-0

${ }^{\star}$ Swertz, C. (2018). Digitale Grundbildung im Pilotversuch. Medienimpulse, 56(3).

Tippelt, R. \& Schmidt-Hertha, B. (Hrsg.). (2018). Springer Reference Sozialwissenschaften. Handbuch Bildungsforschung. Band 2. Springer VS. https://doi.org/10.1007/978-3-53119981-8

*Tondeur, J., van Braak, J., Ertmer, P. A. \& Ottenbreit-Leftwich, A. (2017). Understanding the relationship between teachers' pedagogical beliefs and technology use in education: A systematic review of qualitative evidence. Educational Technology Research and Development, 65(3), 555-575. https://doi.org/10.1007/s11423-016-9481-2

${ }^{*}$ Tsouccas, L. F. \& Meletiou-Mavrotheris, M. (2019). Enhancing In-Service Primary Teachers' Technological, Pedagogical and Content Knowledge on Mobile Mathematics Learning. International Journal of Mobile and Blended Learning, 11(3), 1-18. https:// doi.org/10.4018/IJMBL.2019070101

*van de Oudeweetering, K. \& Voogt, J. (2018). Teachers' Conceptualization and Enactment of Twenty-First Century Competences: Exploring Dimensions for New Curricula. Curriculum Journal, 29(1), 116-133. https://doi.org/10.1080/09585176.2017.1369136

*Voogt, J., Knezek, G., Cox, M., Knezek, D. \& Brummelhuis, A. (2011). Under which conditions does ICT have a positive effect on teaching and learning? A Call to Action. Journal of Computer Assisted Learning, 29(1), 4-14. https://doi.org/10.1111/j.13652729.2011.00453.x

*Vrasidas, C. (2015). The Rhetoric of Reform and Teachers' Use of ICT. British Journal of Educational Technology, 46(2), 370-380. https://doi.org/10.1111/bjet.12149

*Wake, D. \& Mills, M. (2018). Edcamp: Listening to the Voices of Teachers. Issues in Teacher Education, 27(3), 90-106. http://www.itejournal.org/index.html

${ }^{\star}$ Wang, S.-K., Hsu, H.-Y., Campbell, T., Coster, D. C. \& Longhurst, M. (2014). An Investigation of Middle School Science Teachers and Students Use of Technology inside and outside of Classrooms: Considering Whether Digital Natives Are More Technology Savvy than Their Teachers. Educational Technology Research and Development, 62(6), 637-662. https://doi.org/10.1007/s11423-014-9355-4

*Ward, L. \& Parr, J. M. (2010). Revisiting and Reframing Use: Implications for the Integration of ICT. Computers \& Education, 54(1), 113-122. https://doi.org/10.1016/j. compedu.2009.07.011 
${ }^{*}$ Willis, R. L., Lynch, D., Fradale, P. \& Yeigh, T. (2019). Influences on Purposeful Implementation of ICT into the Classroom: An Exploratory Study of K-12 Teachers. Education and Information Technologies, 24(1), 63-77. https://doi.org/10.1007/s10639018-9760-0

*Yildiz, C. (2018). Examination of Middle School Mathematics Teachers' Experiences of Using a Smart Phone. Online Submission.

*Zhang, Y. (2019). Investigating K-12 Teachers' Use of Electronic Board in the Classroom in the Central South of United States. Education and Information Technologies, 24(1), 825-841. https://doi.org/10.1007/s10639-018-9800-9

*Zierer, K. (2015). Pädagogische Mythen. Vermeintliche und tatsächliche Erkenntnisse der empirischen Forschung. Schulmagazin 5-10, 83(12), 7-10. http://www.oldenbourgklick.de/zeitschriften/schulmagazin-5-10/2015-12/paedagogische-mythen 
Marcel Capparozza \& Gabriele Irle

\title{
Lehrerausbildende als Akteure für die Digitalisierung in der Lehrerbildung: Ein Review
}

\begin{abstract}
Im Fokus dieses Reviews stehen Hochschullehrende sowie Mentorinnen und Mentoren als Akteure für die Digitalisierung in der Ausbildung von Lehrkräften. Durch eine deutschund englischsprachige Literaturrecherche in FIS-Bildung, ERIC, Google Scholar sowie der Deutschen Nationalbibliothek werden unter Berücksichtigung der Qualität 15 Studien (2015-2019) zu Lehrerausbildenden ausgewählt und systematisch aufbereitet. Dabei werden drei zentrale Themenfelder fokussiert: 1) Kompetenzen von Lehrerausbildenden, die für das Lehren mit digitalen Medien und zur Vorbereitung der angehenden Lehrkräfte auf das Unterrichten mit digitalen Medien benötigt werden, 2) individuelle Faktoren (z. B. Einstellungen), die den Einsatz digitaler Medien in der Lehre begünstigen sowie 3) die Rolle der institutionellen Unterstützung von Lehrerausbildenden beim Kompetenzerwerb für den Einsatz digitaler Medien. Abschließend werden Empfehlungen für die Forschung und die Lehrerbildung im deutschsprachigen Raum abgeleitet.
\end{abstract}

Schlüsselwörter: Lehrerbildung, Lehramtsstudiengang, Lehrende, digitale Medien, Digitalisierung, Kompetenzen, Professionalisierung, Critical Review

\section{Teacher Educators as Facilitators of Technology Integration in Teacher Education: a Review}

This review presents an overview of research literature on teacher educators and mentor teachers as facilitators of technology integration in teacher education. A literature search that was conducted in ERIC, Google Scholar, FIS-Bildung, and the German National Library, yielded 15 relevant research articles (2015-2019). These research articles are systematically analysed, taking study quality into account. Three domains are identified: 1) competences of teacher educators required for teaching with technology and for preparing pre-service teachers to integrate technology in education, 2) individual factors (e.g. attitudes) that foster technology integration practices of teacher educators, and 3) the role of institutional support for teacher educators in acquiring competences for the use of technology. Finally, recommendations for future research and teacher education practices in German-speaking countries are discussed.

Keywords: teacher education, teacher educators, educational technology, technology integration, pedagogical content knowledge, faculty development, critical review

\section{Einleitung}

Lehrkräfte sollen digitale Medien in den Unterricht integrieren, die Umsetzung im Unterricht reflektieren und Schülerinnen und Schüler auf das Leben in einer zuneh- 
mend digital geprägten Welt vorbereiten (Kultusministerkonferenz, 2017; Starkey, 2019, S. 12). Für die Aneignung dieser Fähigkeiten bei angehenden Lehrkräften spielen Hochschullehrende in den Lehramtsstudiengängen sowie Mentorinnen und Mentoren in praktischen Ausbildungsphasen eine wichtige Rolle.

In diesem Review wird auf Grundlage von empirischen Studien ein Überblick über Lehrerausbildende im Zusammenhang mit der Digitalisierung in der Lehrerbildung gegeben. Im Zentrum des Interesses stehen dabei die Fragen, welche digitalisierungsbezogenen Kompetenzen Lehrerausbildende besitzen sollten und welche individuellen Faktoren dazu führen, dass digitale Medien in der Ausbildung von Lehrkräften eingesetzt werden. Außerdem wird in dem Review thematisiert, wie Lehrerausbildende durch ihre Institutionen dabei unterstützt werden können, digitale Medien in die Lehre zu integrieren und ihre Kompetenzen zu erweitern.

\subsection{Neue Anforderungen an Schule und Lehrerbildung}

Durch den digitalen Wandel entstehen neue Anforderungen an Gesellschaft, Schule und Lehrerbildung (Petko, Döbeli Honegger \& Prasse, 2018). Die Qualität der Ausbildung von Lehrkräften kann dabei langfristig schulische Entwicklungen positiv beeinflussen. In der International Computer and Information Literacy Study 2018 (ICILS) wurde deutlich, dass Lehrkräfte in Deutschland im internationalen Vergleich nach wie vor selten digitale Medien im Unterricht einsetzen (Drossel, Eickelmann, Schaumburg \& Labusch, 2019). Dadurch werden Potenziale von digitalen Medien, wie z.B. die Nutzung multimedialer Darstellungen, adaptiver Lernangebote oder neuer Möglichkeiten der Kommunikation und Zusammenarbeit (Scheiter, 2017), zu selten im Unterricht ausgeschöpft. Neben der technischen Ausstattung benötigen Lehrkräfte für eine erfolgreiche Medienintegration im Unterricht ausreichende Kompetenzen sowie eine positive Einstellung zu digitalen Medien (Knezek \& Christensen, 2016; Petko, 2012). Für die Umsetzung im Unterricht sollten Lehrkräfte dabei technisches, pädagogisches und fachwissenschaftliches Wissen miteinander verknüpfen können (Mishra \& Koehler, 2006). Den Bildungswissenschaften und Fachdidaktiken in der Lehrerbildung kann sowohl zur Förderung dieser Kompetenzen als auch für den Aufbau einer positiven Haltung eine wichtige Rolle zugeschrieben werden (Kay, 2006; Wang, Schmidt-Crawford \& Jin, 2018).

Um angehende Lehrkräfte auf das Unterrichten mit digitalen Medien vorzubereiten, ist deren Unterstützung während der Ausbildung sowohl auf institutioneller als auch auf individueller Ebene notwendig (Tondeur et al., 2012). Einerseits kann auf Ebene der Hochschulen für die Lehrerbildung in Deutschland festgestellt werden, dass neue innovative Formate entwickelt werden (Gemeinsame Wissenschaftskonferenz, 2019; Goertz \& Baeßler, 2018). Andererseits fehlt an vielen Standorten eine verbindliche Verankerung von Inhalten zum Umgang mit digitalen Medien in den Curricula (Brinkmann, Müller, Scholz \& Siekmann, 2018). 
Für die Umsetzung vor Ort kommt Lehrerausbildenden eine entscheidende Bedeutung zu. Als Vorbilder spielen Lehrerausbildende auf der individuellen Ebene indirekt eine wichtige Rolle für die Digitalisierung an Schulen: Sie unterrichten angehende Lehrkräfte, die wiederum nach ihrer Ausbildung digitale Medien im Unterricht einsetzen und thematisieren (Uerz, Volman \& Kral, 2018, S. 13). Die Professionalisierung der Lehrerausbildenden gilt auch deshalb als eine zentrale Herausforderung für die Entwicklung innovativer und nachhaltiger Ansätze für die Digitalisierung in der Lehrerbildung (van Ackeren et al., 2019). In ihrem Review stellten Uerz et al. (2018) fest, dass auf Grundlage der ausgewerteten Studien (2005-2014) technische Kompetenzen, pädagogische Kompetenzen, Kompetenzen zur Innovation und Weiterbildung sowie eine positive Einstellung zum Einsatz digitaler Medien als zentrale Kompetenzen von Lehrerausbildenden identifiziert werden können.

\subsection{Fragestellung des Reviews}

Mithilfe dieses Reviews wird der Forschungsstand zu Hochschullehrenden sowie Mentorinnen und Mentoren im Kontext der Digitalisierung in der Ausbildung von Lehrkräften zusammengefasst. Die Grundlage der Literaturrecherche bildeten drei Fragestellungen:

1) Welche Kompetenzen benötigen Lehrerausbildende, um angehende Lehrkräfte auf das Unterrichten mit digitalen Medien vorzubereiten?

2) Welche Einstellungen und Überzeugungen führen dazu, dass Lehrerausbildende digitale Medien in der Lehre einsetzen?

3) Wie gestaltet sich die Aus- und Weiterbildung in Bezug auf die Förderung der digitalen Kompetenzen von Lehrerausbildenden?

\section{Methode}

Das methodische Vorgehen dieser Studie ist angelehnt an das Verfahren von Critical Reviews. Typisch für Critical Reviews ist, dass sie auf der Grundlage einer systematischen Literaturrecherche die wichtigsten Erkenntnisse aus der Forschung zu einer konkreten Fragestellung identifizieren und in narrativer Form darstellen (Grant \& Booth, 2009).

\subsection{Suchstrategie}

Die Literaturrecherche wurde von April bis Mai 2019 am DIPF | Leibniz-Institut für Bildungsforschung und Bildungsinformation in den Datenbanken FISBildung und ERIC durchgeführt (vgl. Wilmers, Anda, Keller, Kerres \& Getto in diesem Sammelband). Die Suche in beiden Datenbanken basierte auf der 
Kombination von deutsch- und englischsprachigen Schlagwörtern bzw. Freitextsuchen zum Bildungssektor (z. B. ${ }^{\star}$ lehramt ${ }^{\star}$, teacher education), zum Personal (z.B. Hochschullehrer, teacher educator) und zur Digitalisierung (z.B. Neue Medien, Technology Uses in Education). Diese Schlagwortkombinationen wurden mit Schlagwörtern zu Kompetenzen (z.B. Medienkompetenz, technological literacy), zu Einstellungen (z.B. Einstellung*, belief*) und zur Gestaltung der Aus- und Fortbildung (z. B. Fortbildung, skill development) verknüpft. Um kontextbildende Literatur zu finden, wurden zusätzlich auch Begriffe zu Entwicklungen (z.B. Bildungspolitik, transformation) mit den anderen Suchbegriffen verbunden. Weitere schlagwortbasierte Suchen in Google Scholar sowie der Deutschen Nationalbibliothek ergänzten die Suchergebnisse.

Nach dem Screening der Titel und Abstracts sowie der Volltextprüfung (vgl. 2.2), fand in den Literaturverzeichnissen der ausgewählten Studien eine zusätzliche Recherche nach weiteren möglichen passenden Studien statt. Die Wahl fiel deshalb auf dieses Verfahren, weil es nach Giustini (2019, S. 117) vielversprechend ist, um durch die verwendeten Schlagwörter und Datenbanken im Prozess der Literaturrecherche nicht abgedeckte Studien zu einem Thema $\mathrm{zu}$ finden.

\subsection{Auswahl der Studien}

Bei der Auswahl der Studien wurde sowohl die Passung zu den Fragestellungen als auch die Qualität der Studien berücksichtigt. Anknüpfend an den von Uerz et al. (2018) abgedeckten Zeitraum wurden Studien im Kontext Digitalisierung und Lehrerbildung berücksichtigt, die seit 2015 erschienen sind. Zur Vorauswahl dienten im ersten Screening die Titel und Abstracts der Suchergebnisse. Als Ausschlusskriterium diente ein fehlender Bezug zum Bildungssektor, zu Digitalisierung oder zur Fragestellung. Es wurden ausschließlich Primärerhebungen berücksichtigt. Ein Überblick über die Ein- und Ausschlusskriterien wird in Tabelle 1 gegeben.

Tabelle 1: Ein- und Ausschlusskriterien für die Aufnahme der Studien

\begin{tabular}{|c|c|c|}
\hline Kriterium & Einschluss & Ausschluss \\
\hline $\begin{array}{l}\text { Methodischer } \\
\text { Zugang }\end{array}$ & $\begin{array}{l}\text { Primärerhebungen (quantitativ, } \\
\text { qualitativ, mixed methods) }\end{array}$ & $\begin{array}{l}\text { alle weiteren (z. B. Reviews, Theoriebei- } \\
\text { träge) }\end{array}$ \\
\hline Fokus & $\begin{array}{l}\text { Hochschullehrende bzw. Mentorin- } \\
\text { nen oder Mentoren im Kontext } \\
\text { Digitalisierung und Lehrerbildung }\end{array}$ & $\begin{array}{l}\text { fehlender Bezug zu Digitalisierung oder } \\
\text { Lehrerbildung; Schwerpunkt auf Lehre, } \\
\text { eingesetzten Medien oder Studierenden; } \\
\text { reine Online-Lehre }\end{array}$ \\
\hline Zeitraum & $01 / 2015-05 / 2019$ & früher und später erschienene Beiträge \\
\hline Dokumententyp & $\begin{array}{l}\text { Zeitschriftenbeiträge, Beiträge in } \\
\text { Sammelwerken, Monographien, } \\
\text { Konferenzbeiträge }\end{array}$ & Hochschulschriften, graue Literatur \\
\hline Sprache & Deutsch, Englisch & alle weiteren \\
\hline
\end{tabular}


Alle Studien mit Passung zur Fragestellung wurden einer Qualitätsbewertung unterzogen. Mithilfe vorab festgelegter Kriterien erfolgte eine Einschätzung, wie nah eine Studie an der Realität ist (Validität), wie zuverlässig die Ergebnisse einzuschätzen sind (Reliabilität) und wie hoch die Anwendbarkeit bzw. Generalisierbarkeit der Ergebnisse ist (Booth, Sutton \& Papaioannou, 2016, S. 154-155). Als Kriterien für eine hohe Validität wurden eine ausreichende Berücksichtigung des Forschungsstandes, die Replizierbarkeit der verwendeten Methode sowie die Nachvollziehbarkeit der Ergebnisse und des Diskussionsteils festgelegt. Durch Bewertung des Designs der Studie, der verwendeten Instrumente zur Datenerhebung sowie der Datenauswertung konnten Aussagen über die Zuverlässigkeit des methodischen Vorgehens getroffen werden. Eine hohe Generalisierbarkeit der Studien wurde angenommen, wenn die Stichprobenziehung theoriebasiert oder randomisiert erfolgte und wenn das Bildungssystem in dem Land, in dem die Studie durchgeführt wurde, möglichst große Schnittmengen mit dem deutschsprachigen Bildungssystem teilt.

In Anlehnung an Rost (2013) erfolgte die Einschätzung der Qualität entlang der Abschnitte Einleitung, Theorie, Methode, Ergebnisse und Diskussion. Für jedes Qualitätskriterium wurden Indikatoren festgelegt (z.B. Bewertung der Stichprobenwahl: 2 Punkte $=$ randomisierte und/oder theoriebasierte Auswahl, 1 Punkt $=$ mehrere Hochschulen berücksichtigt, 0 Punkte $=$ Ad-Hoc-Stichprobe) . Durch die Indikatoren sollte eine möglichst objektive Bewertung der Qualität erfolgen. Der Mittelwert aller Qualitätskriterien ergab einen Anhaltspunkt bezüglich der Gesamtqualität der Studien. Alle Studien, deren Mittelwert aller Qualitätskriterien im oberen Terzil lag $(M>1.33)$, wurden aufgenommen. Zudem wurden Studien aufgrund der mangelnden Qualität nicht berücksichtigt, wenn deren Mittelwert im unteren Terzil $(M<0.67)$ lag. Studien mit methodischen Mängeln (z.B. fehlende Nachvollziehbarkeit des Vorgehens, Verwendung nicht reliabler Skalen) wurden auf diese Weise ausgeschlossen. Einschränkungen in der methodischen Qualität, die relevant für die Interpretation der aufgenommenen Studien sind, werden in der Ergebnisdarstellung genannt. Studien mit einem Mittelwert zwischen dem obersten und untersten Terzil wurden vor allem auf Grundlage ihrer Relevanz für die Beantwortung der Fragestellung ausgewählt (Grant \& Booth, 2009, S. 94). In den aufgenommenen Studien wurden zentrale übergreifende Themen identifiziert (vgl. 3.1.3) und als Ergebnisse des Reviews ausgewertet. 


\section{Ergebnisse}

Im Folgenden wird ein Überblick über die aufgenommenen Studien (Auswahlprozess, Qualität und thematische Schwerpunkte) gegeben. Anschließend werden die durch die Analyse der Studien identifizierten Themenbereiche dargestellt. Ein zentrales Thema in den Studien sind Kompetenzen von Lehrerausbildenden. Außerdem werden individuelle Einflussfaktoren (z.B. Einstellungen) sowie externe Einflussfaktoren auf Lehrerausbildende vorgestellt.

\subsection{Beschreibung der aufgenommenen Studien}

\subsubsection{Auswahlprozess}

Eine Überprüfung der Titel und Abstracts fand bei 1193 Suchergebnissen statt, die seit 2015 veröffentlicht wurden. Durch den Ausschluss von Studien mit einem anderen Fokus bzw. unpassendem Dokumententyp reduzierte sich die Zahl für die Volltextprüfung auf 74. Mithilfe der Volltextprüfung erfolgte der Ausschluss von weiteren 60 Studien. Gründe hierfür lagen insbesondere in der Passung auf die Fragestellung und der Qualität der Studien. Auf Grundlage der Schneeballsuche in den Literaturverzeichnissen der aufgenommenen Studien wurde eine zusätzliche Studie (Tondeur, Aesaert, Prestridge \& Consuegra, 2018) hinzugefügt. Der detaillierte Auswahlprozess ist in Abbildung 1 dargestellt.

Alle aufgenommenen Studien wurden in Zeitschriften mit Peer-Review veröffentlicht. Auffällig war der geringe Anteil an Studien zur Digitalisierung und Lehrerbildung in den Suchergebnissen, in denen die Personengruppe der Lehrerausbildenden im Kontext Digitalisierung überhaupt als Schwerpunktthema thematisiert wurde: Von 548 Suchergebnissen mit Bezug zu Lehrerbildung und Digitalisierung wurden letztendlich lediglich 24 einer Qualitätsbewertung unterzogen (4.4\%). Viel häufiger standen in Studien zur Digitalisierung in der Lehrerbildung Fragestellungen im Fokus, die sich z.B. den Kompetenzen und Einstellungen von Studierenden oder der Gestaltung und Evaluation von Lehrveranstaltungen widmeten. 


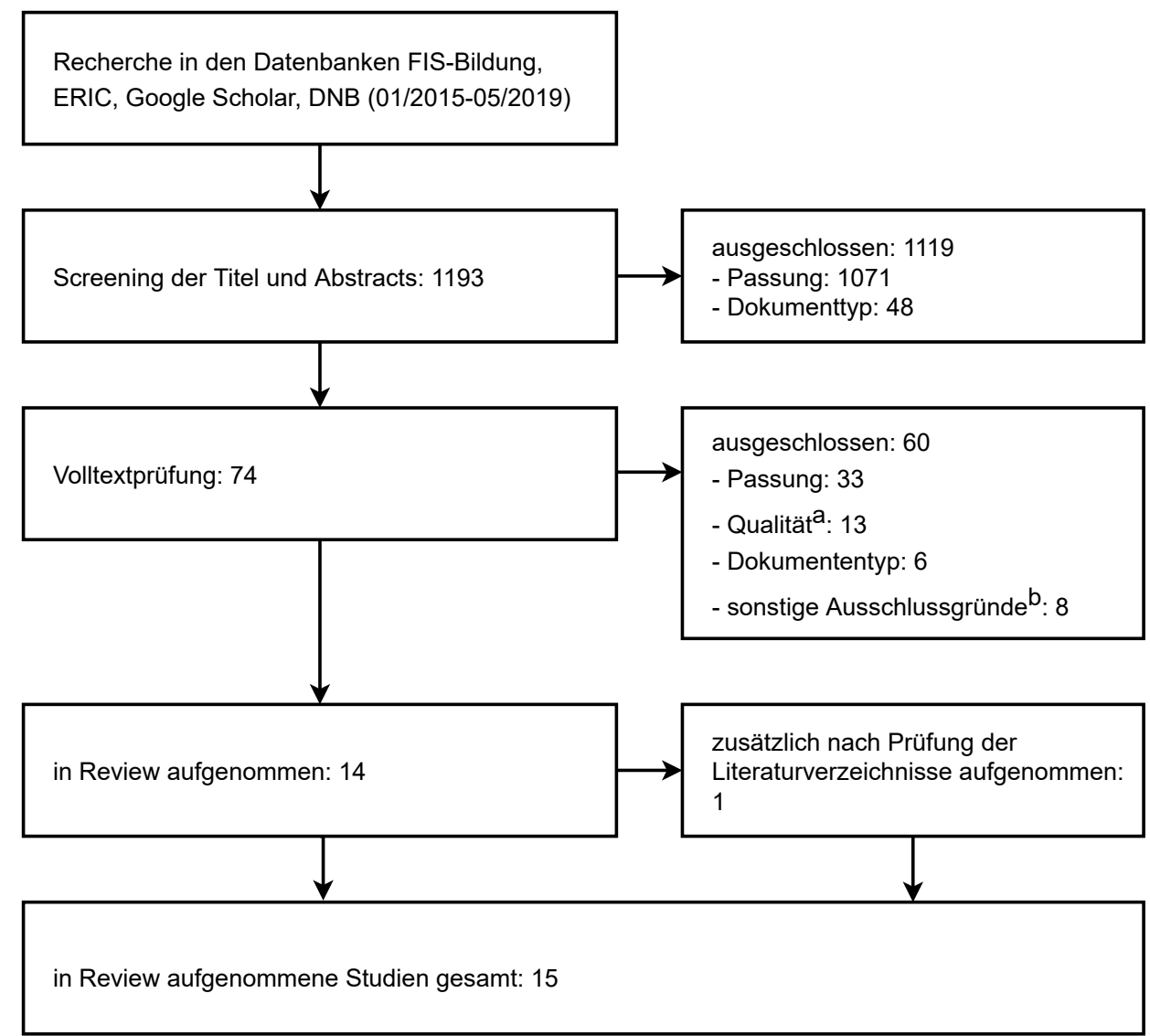

Abbildung 1: Überblick über den Auswahlprozess

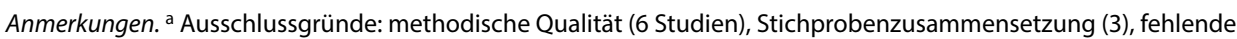

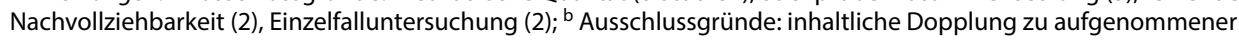
Studie (2), vor 2012 durchgeführte Erhebung (2), Pilotierung (3), Sprache (1).

\subsubsection{Qualität der aufgenommenen Studien}

Um die Qualität der aufgenommenen Studien abschätzen zu können, wurden für jede Studie die Mittelwerte der neun Qualitätskriterien berechnet (vgl. 2.2). Von den 15 aufgenommen Studien waren 11 von relativ hoher Qualität (d.h. $M \geq 1.34$ ). Die vier übrigen Studien wurden vor allem aufgrund ihrer Bedeutung für die Beantwortung der Fragestellung aufgenommen. Die Aufnahme von drei dieser Studien (Instefjord \& Munthe, 2017; Liu, Tsai \& Huang, 2015; Nelson, 2017) erfolgte, weil in diesen Mentorinnen und Mentoren thematisiert wurden. Die Studie von Avidov-Ungar und Forkosh-Baruch (2018) wurde ausgewählt, weil sie die Studie mit der höchsten Qualität war, in der der Einfluss der Digitalisierung auf die berufliche Identität von Lehrerausbildenden beschrieben wurde. Ein Überblick über die 
Indikatoren für Qualität der in das Review aufgenommenen Studien findet sich in Tabelle 2. Zur ausreichenden Berücksichtigung des Forschungsstands wurde in sechs Fällen lediglich Theorien genannt, aber es war keine systematische und umfassende Literaturrecherche erkennbar. Die methodische Qualität und Nachvollziehbarkeit der Studien war überwiegend gut, wobei sich in einigen Studien auch Schwächen zeigten (z. B. für die gewählten quantitativen Verfahren ungeeignete Stichprobengrößen, Angaben der Summen- anstatt der Mittelwerte bei Skalen). Auffällig ist außerdem, dass die Generalisierbarkeit der Ergebnisse eingeschränkt ist. Erstens liegt dies daran, dass in einem Drittel der aufgenommenen Studien Ad-hoc-Stichproben genutzt wurden. Zweitens wurde keine der Studien im deutschsprachigen Raum durchgeführt. Immerhin wurden sechs Studien innerhalb Europas durchgeführt, wodurch zumindest eine vergleichsweise höhere kulturelle Nähe sowie durch den BolognaProzess angeglichene Hochschulsysteme erwartet werden können.

Tabelle 2: Übersicht über die Indikatoren für die Qualität aller aufgenommenen Studien ( $k=15)$

\begin{tabular}{lccc}
\hline \multicolumn{1}{c}{ Qualitätskriterium } & \multicolumn{3}{c}{ Anzahl der Studien } \\
\cline { 2 - 4 } & erfüllt & $\begin{array}{c}\text { teilweise } \\
\text { erfüllt }\end{array}$ & $\begin{array}{c}\text { nicht } \\
\text { erfüllt }\end{array}$ \\
\hline Ausr. Berücksichtigung des Forschungsstandes & 9 & 6 & 0 \\
Replizierbarkeit des methodischen Vorgehens & 12 & 3 & 0 \\
Nachvollziehbarkeit der Ergebnisse & 11 & 4 & 0 \\
Nachvollziehbarkeit des Diskussionsteils & 14 & 1 & 0 \\
positive Bewertung des Studiendesigns & 14 & 1 & 0 \\
positive Bewertung der Instrumente & 9 & 6 & 0 \\
Angemessenheit der Datenauswertung & 10 & 5 & 0 \\
Generalisierbarkeit der Stichprobenauswahl & 8 & 2 & 5 \\
Generalisierbarkeit auf deutsches Bildungssystem & 0 & 6 & 9 \\
\hline
\end{tabular}

\subsubsection{Thematische Schwerpunkte der Studien}

Insgesamt wurden in zehn Studien quantitative Fragebogenerhebungen durchgeführt. Als Stichprobe dienten dabei Lehrerausbildende (4 Studien), Lehramtsstudierende (4 Studien) sowie beide Zielgruppen (2 Studien). Der Einsatz qualitativer Methoden beinhaltete verschiedene methodische Zugänge: Fragebögen mit offenen Fragen, Interviews, Fokusgruppeninterviews, Dokumentenanalysen und Beobachtungen von Unterrichtssituationen (3 Studien). Hier wurden in allen Fällen Lehrerausbildende befragt, in einem Fall zusätzlich auch die angehenden Lehrkräfte. Darüber hinaus finden sich in der Auswahl Delphi-Befragungen, in denen Expertinnen und Experten zu einem spezifischen Thema befragt wurden (2 Studien). In Tabelle 3 wird ein Überblick über alle 15 aufgenommenen Studien gegeben. 
Lehrerausbildende als Akteure für die Digitalisierung in der Lehrerbildung

Tabelle 3: Überblick über die aufgenommenen Studien

\begin{tabular}{|c|c|c|c|c|c|c|c|c|}
\hline \multirow[t]{2}{*}{ Autoren } & \multirow[t]{2}{*}{ Design } & \multirow[t]{2}{*}{ Instrument } & \multirow[t]{2}{*}{ Land } & \multirow[t]{2}{*}{$n$} & \multirow[t]{2}{*}{ Stichprobe } & \multicolumn{3}{|c|}{ Schwerpunkt } \\
\hline & & & & & & (1) & (2) & (3) \\
\hline $\begin{array}{l}\text { Avidov-Ungar \& } \\
\text { Forkosh-Baruch, } \\
2018\end{array}$ & qualitativ & I & Israel & 27 & Lehrende & & & $x$ \\
\hline Baran et al., 2019 & quantitativ & $\mathrm{FB}$ & Türkei & 215 & Studierende & $x$ & & \\
\hline Becuwe et al., 2017 & mixed & Delphi & Belgien & 36 & Experten & & & $x$ \\
\hline Cuhadar, 2018 & quantitativ & FB & Türkei & 832 & Studierende & $x$ & & \\
\hline Foulger et al., 2017 & qualitativ & Delphi & $\begin{array}{l}\text { USA, } \\
\text { Australien }\end{array}$ & 17 & Lehrende & $x$ & & \\
\hline $\begin{array}{l}\text { Instefjord \& Munthe, } \\
2017\end{array}$ & quantitativ & $\mathrm{FB}$ & Norwegen & $\begin{array}{l}387 \\
654 \\
288\end{array}$ & $\begin{array}{l}\text { Lehrende } \\
\text { Studierende } \\
\text { Mentoren }\end{array}$ & $\mathrm{x}$ & & $\mathrm{x}$ \\
\hline $\begin{array}{l}\text { Kalonde \& Mousa, } \\
2016\end{array}$ & qualitativ & $\mathrm{FB}, \mathrm{I}$ & USA & 90 & Lehrende & & $x$ & $x$ \\
\hline Liu et al., 2015 & qualitativ & DA, FGI, B & Taiwan & $\begin{array}{l}3 \\
3\end{array}$ & $\begin{array}{l}\text { Mentoren } \\
\text { Mentees }\end{array}$ & $x$ & & $x$ \\
\hline Nelson, 2017 & quantitativ & FB & USA & $\begin{array}{l}48 \\
77\end{array}$ & $\begin{array}{l}\text { Mentoren } \\
\text { Mentees }\end{array}$ & $\mathrm{x}$ & & \\
\hline Nelson et al., 2019 & quantitativ & $\mathrm{FB}$ & USA & 806 & Lehrende & $\mathrm{x}$ & & $x$ \\
\hline Taimalu \& Luik, 2019 & quantitativ & FB & Estland & 54 & Lehrende & $\mathrm{x}$ & $x$ & \\
\hline Tondeur et al., 2019 & quantitativ & $\mathrm{FB}$ & Belgien & 284 & Lehrende & $\mathrm{x}$ & $\mathrm{x}$ & \\
\hline Tondeur et al., 2018 & quantitativ & FB & Belgien & 931 & Studierende & $\mathrm{x}$ & & \\
\hline Tondeur et al., 2017 & quantitativ & $\mathrm{FB}$ & Belgien & 688 & Studierende & $x$ & & \\
\hline Voithofer et al., 2019 & quantitativ & FB & USA & 842 & Lehrende & $\mathrm{x}$ & & $\mathrm{x}$ \\
\hline
\end{tabular}

Anmerkungen. Abkürzungen: $\mathrm{FB}=$ Fragebogen, I = Interview, FGI = Fokusgruppeninterview, DA =

Dokumentenanalyse, B = Beobachtung, Delphi = Delphi-Befragung; Schwerpunkte der Studien: (1) Kompetenzen von Lehrerausbildenden, (2) individuelle Einflussfaktoren, (3) institutionelle Unterstützung.

Am häufigsten thematisierten die Autorinnen und Autoren der Studien Kompetenzen von Lehrerausbildenden (Fragestellung 1). Auch Einstellungen und Überzeugungen (Fragestellung 2) wurden thematisiert. Diese werden im Folgenden gemeinsam mit weiteren identifizierten Einflussfaktoren auf den Einsatz digitaler Medien unter individuelle Einflussfaktoren zusammengefasst. Abschließend werden die Rolle der institutionellen Unterstützung sowie zwei Weiterbildungsmaßnahmen für Lehrerausbildende (Fragestellung 3) beschrieben. 


\subsection{Kompetenzen von Lehrerausbildenden}

Die für den Einsatz digitaler Medien und die Förderung der mediendidaktischen Fähigkeiten angehender Lehrkräfte benötigten Kompetenzen und Fähigkeiten von Lehrerausbildenden werden in den aufgenommenen Studien über drei Modelle beschrieben. Eine Rolle in der Forschung zu Lehrerausbildenden spielt das TPACKModell, das Mishra und Koehler (2006) ursprünglich als Modell zur Erweiterung der Komponenten des Professionswissens von Lehrkräften entwickelten. Das zweite Modell, mit dem vor allem lehramtsspezifische Handlungskompetenzen von Lehrerausbildenden operationalisiert werden, sind die SQD-Strategien (Tondeur et al., 2012; Tondeur, van Braak, Siddiq \& Scherer, 2016). Zu beiden Modellen wird im Folgenden der Forschungsstand dargestellt. Abschließend folgt die Beschreibung einer Studie zu der Genese der Teacher Educator Technology Competencies (Foulger, Graziano, Schmidt-Crawford \& Slykhuis, 2017). Diese stellen eine Weiterentwicklung und Spezifizierung des Kompetenzbegriffs für Lehrerausbildende dar.

\subsubsection{TPACK-Modell: Technologiebezogenes fachdidaktisches Wissen}

Ursprünglich wurde das TPACK-Modell konzipiert, um das Professionswissen von Lehrkräften um eine technologische Komponente zu erweitern. Für die Integration digitaler Medien in den Unterricht sollen Lehrkräfte demnach sowohl über technisches (Technology Knowledge) als auch über pädagogisches (Pedagogy Knowledge) und fachliches Wissen (Content Knowledge) verfügen und dieses Wissen als technologiebezogenes fachdidaktisches Wissen verknüpfen (Technological Pedagogical Content Knowledge; Mishra \& Koehler, 2006; Schmid \& Petko, 2020). In der neuesten Erweiterung ergänzte Mishra (2019) das Wissen über den Kontext (Contextual Knowledge) als vierte Wissenskomponente (vgl. Abbildung 2). In vier der aufgenommenen Studien wird das TPACK-Modell für die Operationalisierung des Wissens von Lehrerausbildenden eingesetzt.

In der Studie von Nelson (2017) findet sich ein positiver Zusammenhang zwischen den TPACK-Kompetenzen der betreuenden Lehrkräfte und den TPACKKompetenzen ihrer Mentees. Diesen Effekt stellte Nelson (2017) allerdings nur unter zwei Bedingungen fest: Erstens mussten die Mentorinnen und Mentoren häufig beim Einsatz von digitalen Medien im Klassenzimmer beobachtet werden und zweitens fand sich der positive Zusammenhang nur, wenn die Mentorinnen und Mentoren ihr eigenes technologiebezogenes fachdidaktisches Wissen (TPACK) hoch einschätzten. Einschränkend für die Interpretation dieses Befunds ist die hohe Zahl von nicht berücksichtigten Befragten zu nennen, zu denen in dieser Studie aufgrund des Designs (zwei Messzeitpunkte, Onlinebefragung der Mentorinnen und Mentoren) nicht alle Daten vollständig vorlagen. 


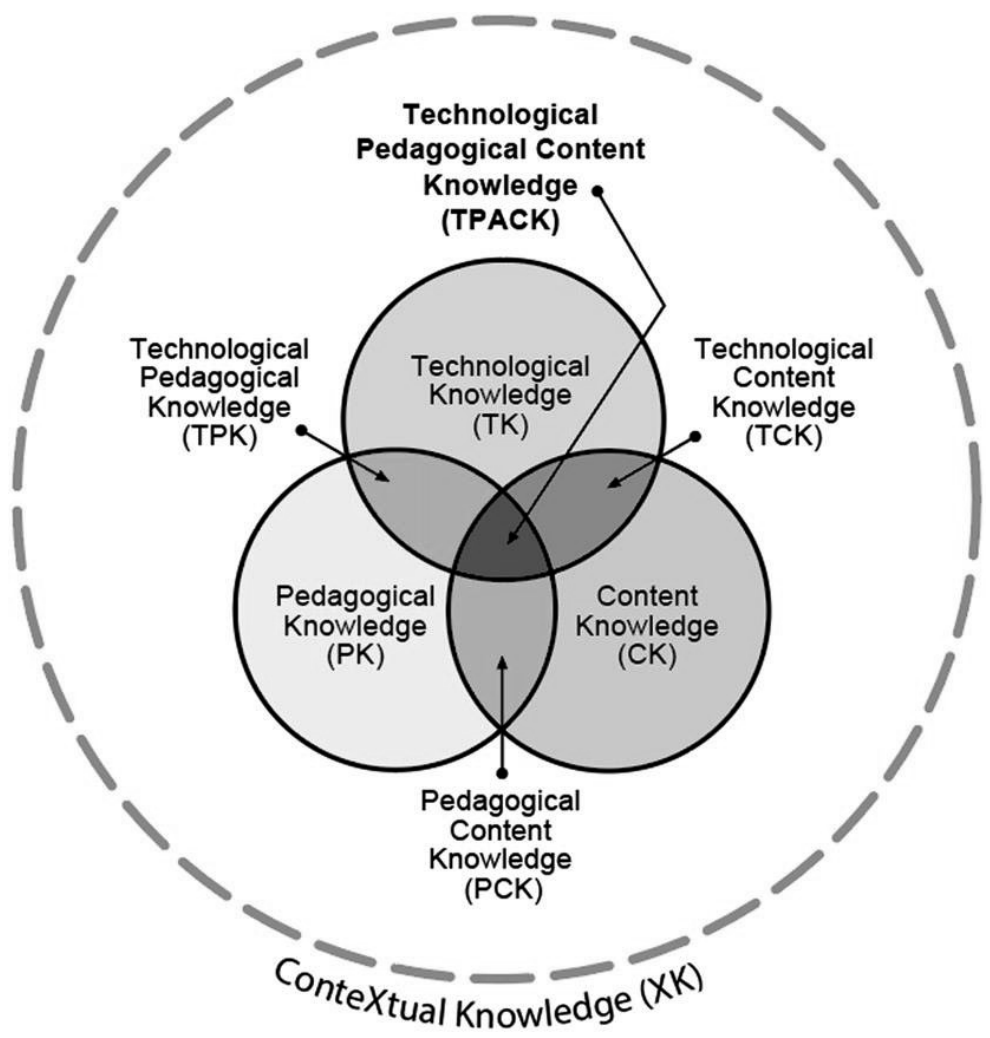

Abbildung 2: Das TPACK-Modell. Quelle: Mishra, 2019, S. 77

Welche Faktoren spielen eine Rolle für die Integration von technischem, pädagogischem und fachwissenschaftlichem Wissen von Lehrerausbildenden? Ein möglicher Einflussfaktor ist das Vorwissen der Lehrerausbildenden zu einzelnen Wissenskomponenten: In der Befragung von Taimalu und Luik (2019) war das selbsteingeschätzte pädagogische Wissen ein signifikanter Prädiktor für TPACK $(\beta=0.24, p<.05)$. Auch technisches Vorwissen spielt eine Rolle: Lehrerausbildende des Fachs Technik setzen in der Lehre ihre TPACK-Kompetenzen eher als Lehrerausbildende mit Fächern wie Englisch, Mathematik oder mit naturwissenschaftlichem Hintergrund ein (Nelson, Voithofer \& Cheng, 2019; Voithofer, Nelson, Han \& Caines, 2019). Der niedrigere TPACK-Wert kann bei Lehrerausbildenden aus den Fächern Mathematik und Englisch durch einen indirekten Effekt aufgrund des niedrigeren technischen Wissens erklärt werden (Nelson et al., 2019).

Ein weiterer möglicher Einflussfaktor auf TPACK ist die Erfahrung. Die Erfahrung in der Hochschullehre erwies sich in der Studie von Voithofer et al. (2019) nicht als signifikanter Prädiktor für TPACK. Bei Nelson et al. (2019) fand sich dagegen ein schwacher positiver Effekt durch die Erfahrung in der Hochschullehre, wenn die beiden Kontrollvariablen Wahrnehmung der technischen Unterstützung und 
technisches Wissen in der Auswertung berücksichtigt wurden. Das bedeutet, dass vor allem die pädagogischen Erfahrungen eine Rolle spielen. Lehrerausbildende, die in der Studie von Voithofer et al. (2019) hohe TPACK-Werte aufwiesen, hatten signifikant mehr schulische Unterrichtserfahrung als Lehrerausbildende, die niedrigere Werte hatten, $t(151)=2.16, p=.032$. Allerdings konnte in der methodisch ähnlich angelegten Befragung von Nelson et al. (2019) kein signifikanter Einfluss der Unterrichtserfahrung gefunden werden. Der Einfluss der Erfahrung auf das technologiebezogene fachdidaktische Wissen (TPACK) scheint auf Grundlage dieser beiden Studien noch nicht abschließend geklärt.

\subsubsection{SQD-Strategien: Vorbereitung angehender Lehrkräfte auf das Unterrichten mit digitalen Medien}

Eine weitere und eher pädagogische Handlungen beschreibende Operationalisierung von Kompetenz sind die sogenannten SQD-Strategien (Synthesis of Qualitative Data; Tondeur et al., 2012). Die SQD-Strategien wurden auf Grundlage der Auswertung von qualitativen Studien erstellt und beinhalten sowohl übergreifende (z.B. Verknüpfung von Theorie und Praxis), institutionelle (z.B. Fort- und Weiterbildung) als auch individuelle Strategien (Tondeur et al., 2012; vgl. Abbildung 3). Aus diesem Modell wurden die individuellen Strategien für die Skalenentwicklung genommen, um Strategien von Lehrerausbildenden auf der Mikroebene abzubilden (Baran, Canbazoglu Bilici, Albayrak Sari \& Tondeur, 2019; Cuhadar, 2018; Tondeur et al., 2016). Lehrerausbildende sollen demnach über mehrere individuelle Handlungskompetenzen verfügen: Sie sollen die Reflektion über das Unterrichten mit digitalen Medien fördern, authentische Erfahrungen ermöglichen, kollaborative Lernszenarien einsetzen, Studierende bei der Planung des Unterrichts mit digitalen Medien unterstützen, kontinuierlich Rückmeldungen geben und Vorbilder für angehende Lehrkräfte sein (Tondeur et al., 2012; Tondeur et al., 2016).

In mehreren Studien fanden sich positive Zusammenhänge zwischen den SQD-Strategien der Lehrerausbildenden und den Kompetenzen der Studierenden. Beispielsweise war in der Studie von Baran et al. (2019) der wahrgenommene Einsatz von SQD-Strategien ein signifikanter Prädiktor für die selbsteingeschätzten TPACK-Kompetenzen der befragten Studierenden, $F(1,213)=87.796, p<.001$. Tondeur et al. (2018) konnten mithilfe einer Mehrebenenanalyse positive Effekte der SQD-Strategien auf die Einschätzung der Studierenden sowohl bezüglich ihrer Kompetenzen zum Einsatz digitaler Medien im Unterricht $(\beta=0.14, p<.05)$ als auch bezüglich ihrer Kompetenzen zur Gestaltung von digitalen Lernumgebungen $(\beta=0.26, p<.05)$ nachweisen. Der Einsatz von SQD-Strategien wird in einer weiteren Studie besonders von Studierenden berichtet, die bereits ein hohes Wissen, positive Einstellungen und eine hohe Selbstwirksamkeitserwartung bezüglich des Einsatzes digitaler Technologien haben (Tondeur, Scherer, Siddiq \& Baran, 2017). 


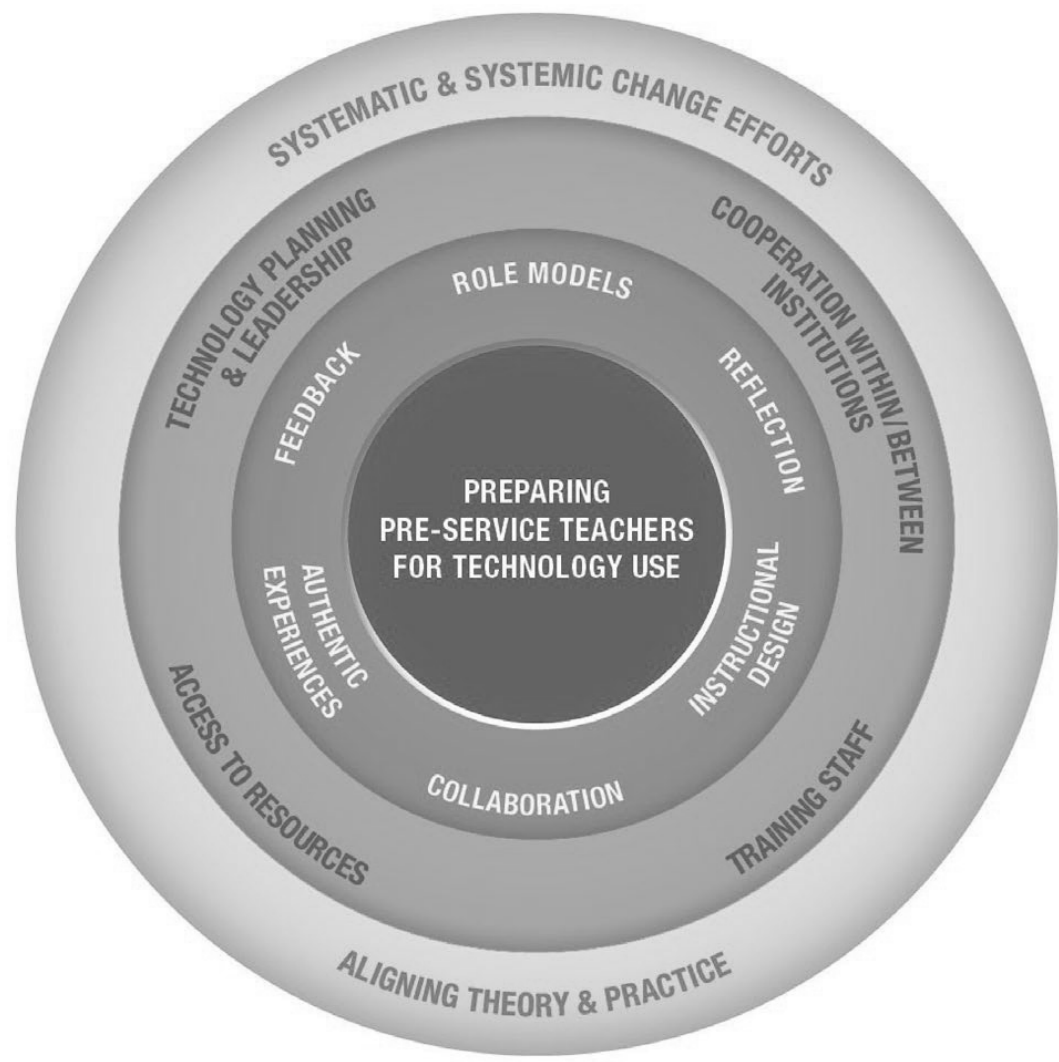

Abbildung 3: SQD-Strategien. Quelle: Tondeur et al., 2012, S. 141

Zusammengefasst deuten die Studienergebnisse darauf hin, dass durch Einsatz der SQD-Strategien mediendidaktische Kompetenzen von angehenden Lehrkräften gefördert werden können.

Eine Strategie zur Vorbereitung von Lehramtsstudierenden auf das Unterrichten mit digitalen Medien ist die Vorbildfunktion. Zur Frage, ob Studierende ihre Lehrerausbildenden bezüglich des Einsatzes von Technologien als Vorbilder sehen, gibt es widersprüchliche Ergebnisse. Im Vergleich zu anderen SQD-Strategien wird in Befragungen von Studierenden der Vorbildfunktion der Lehrerausbildenden ein hoher Wert zugesprochen (Baran et al., 2019; Cuhadar, 2018; Tondeur et al., 2018). In der einzigen Befragung, in der sowohl Lehrerausbildende als auch Studierende direkt zur Vorbildfunktion befragt wurden (Instefjord \& Munthe, 2017), war auf einer Likert-Skala von 1 bis 6 die Selbsteinschätzung der Lehrerausbildenden deutlich höher $(M=3.90, S D=1.19)$ als die Fremdeinschätzung der Studierenden $(M=2.72$, $S D=1.35$ ). Die Ergebnisse der Studien deuten zusammengefasst darauf hin, dass die Vorbildfunktion der Lehrerausbildenden im Vergleich zu anderen Strategien als besonders hoch eingeschätzt wird. Dies könnte damit zusammenhängen, dass die 
Vorbildfunktion unabhängig von den sonstigen eingesetzten Strategien immer beobachtbar ist.

Mentorinnen und Mentoren sollten ebenfalls ein wichtiges Vorbild für angehende Lehrkräfte sein, insbesondere weil sie durch ihre Erfahrung im Unterrichten andere Kompetenzen besitzen als Lehrerausbildende an Hochschulen. Die von Instefjord und Munthe (2017) befragten Mentorinnen und Mentoren schätzen beispielsweise ihre Kompetenz, mit digitalen Medien das Lernen von Schülerinnen und Schüler zu fördern, im Durchschnitt höher ein $(M=4.57, S D=1.09)$ als die befragten Lehrenden an Hochschulen $(M=3.90, S D=1.19)$. Angehende Lehrkräfte können dabei von den pädagogischen Erfahrungen der Mentorinnen und Mentoren profitieren (Liu et al., 2015).

\subsubsection{TETCs-Modell: Teacher Educator Technology Competencies}

Im Reviewprozess wurde außerdem eine Studie identifiziert, in der gezielt eine Weiterentwicklung des Kompetenzbegriffs speziell für Lehrerausbildende in den Blick genommen wurde. Foulger et al. (2017) erstellten mithilfe eines Literaturreviews und einer anschließenden Delphi-Befragung von 17 Lehrerausbildenden eine Liste mit zwölf Teacher Educator Technology Competencies (TETCs; vgl. Abbildung 4). Die TETCs werden mit jeweils zwei bis fünf Indikatoren spezifiziert. Auffällig ist, dass für die beiden Kompetenzen im TETCs-Modell, die von den Befragten als am bedeutendsten eingeschätzt wurden, die Integration von technischem, pädagogischem und fachwissenschaftlichem Wissen (TPACK) eine große Rolle spielt. Gleichzeitig nannten die befragten Lehrerausbildenden aber auch Kompetenzen, die gleichermaßen für Hochschullehrende außerhalb der Lehrerbildung von Bedeutung sind (z.B. Kompetenzmessung, Verwendung von Online-Tools). Anders als TPACK oder die SQD-Strategien ist das TETCs-Modell bisher selten in empirischen Studien zum Einsatz gekommen, da die Entwicklung von Instrumenten noch am Anfang steht. Nach dem im Review abgedeckten Zeitraum wurden Studien veröffentlicht, die darauf hindeuten, dass das Konzept der TETCs zukünftig eine größere Rolle in der Forschung zu Lehrerausbildenden spielen könnte (z. B. Dillon, Chang, Rondeau \& Kim, 2019; Knezek, Christensen \& Furuta, 2019; Thomas, Peterson \& Abebe, 2019).

\subsection{Individuelle Einflussfaktoren auf den Einsatz digitaler Medien}

Zwei Autorenteams (Taimalu \& Luik, 2019; Tondeur et al., 2019) thematisieren die Einstellung der Lehrerausbildenden. Tondeur et al. (2019) konnten mithilfe einer latenten Klassenanalyse zwei Profile von Lehrerausbildenden aus Belgien $(n=284)$ identifizieren. Dabei berücksichtigten sie verschiedene Variablen, die für den Einsatz digitaler Medien in der Ausbildung von Lehrkräften von Bedeutung sind (Einstellung 


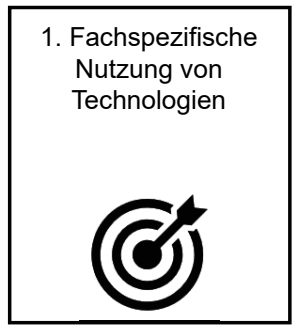

5. Nutzung von Technologie für den Umgang mit heterogenen Lerngruppen

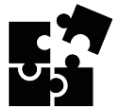

9. Vermittlung von Wissen über Recht, Ethik und sozial verantwortlichen Einsatz

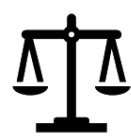

2. Integration pädagogischer Ansätze in die Lehre

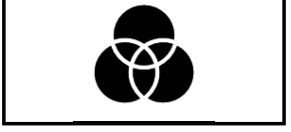

6. Verwendung geeigneter Verfahren zur Messung von Kompetenzen

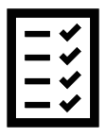

10. Kontinuierliche berufliche Weiterbildung und Netzwerkaktivitäten

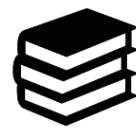

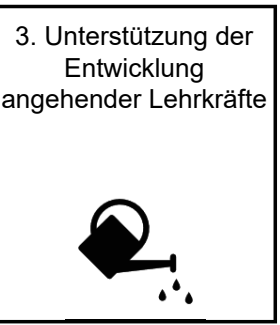

7. Einsatz von Onlineund hybriden Lernumgebungen

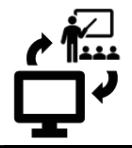

\section{Lehrende als} Vorreiter und Verfechter der Nutzung von Technologien

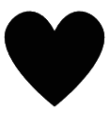

4. Verwendung von Online-Tools zur Verbesserung von Lehr- und Lernprozessen

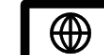

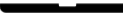

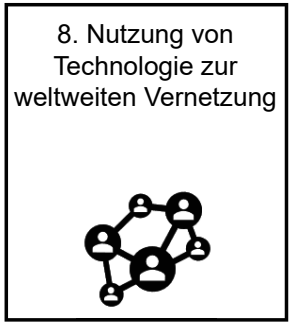

12. Grundlegende technische Problemlösekompetenz

Abbildung 4: Teacher Educator Technology Competencies (eigene Darstellung nach Foulger et al., 2017, eigene Übersetzung)

zu Informations- und Kommunikationstechnologie, Selbstwirksamkeit, Fähigkeit zum abstrakten Denken, informationsbezogene Problemlösekompetenz, Fähigkeit zur Förderung des selbständigen Lernens, Häufigkeit des Einsatzes der SQDStrategien). Das erste Profil bezeichneten Tondeur et al. (2019) als high (Teacher Educator) ICT profile (47.2\% der Befragten), weil dieses Profil in allen Bereichen signifikant höhere Zustimmungswerte hatte als das der Lehrerausbildenden aus dem low (Teacher Educator) ICT profile (52.8\% der Befragten). Lehrerausbildende mit einer positiven Einstellung hatten in dieser Studie also tendenziell auch eine höhere technische und pädagogische Kompetenz.

Ergebnisse zum Zusammenhang zwischen Einstellungen, Kompetenzen und dem tatsächlichen Einsatz digitaler Medien fanden sich nur in einer Studie. In der Befragung von Taimalu und Luik (2019) bestand ein positiver signifikanter Zusammenhang zwischen Überzeugungen zum Mehrwert des Technologieeinsatzes in der Lehre und der selbstwahrgenommenen Kompetenz $(\beta=0.48, p<.001)$. Die selbstwahrgenommene Kompetenz war auch der einzige signifikante direkte Einflussfaktor auf den Einsatz von digitalen Medien in der Lehre $(\beta=0.72, p<.01)$. Eine hohe Überzeugung über den Mehrwert des Technologieeinsatzes führte da- 
gegen (ebenso wie eine hohe Selbstwirksamkeitserwartung) nicht direkt zu mehr Integration von digitalen Medien in die Lehre $(\beta=-0.09, p>.05)$. Taimalu und Luik (2019) fanden zudem einen signifikanten positiven Zusammenhang zwischen konstruktivistischen pädagogischen Konzepten, die eine Ausrichtung der Lehre auf die Studierenden unterstützen, und Überzeugungen zum Mehrwert des Technologieeinsatzes in der Lehre $(\beta=0.27, p<.05)$. Dagegen gab es einen signifikanten negativen Zusammenhang, wenn Lehrerausbildende traditionelle Lehrvorstellungen vertraten, in denen der Fokus nicht auf den Lernenden lag $(\beta=-0.29$, $p<.05)$.

Nicht nur die Einstellung spielt eine Rolle für den Einsatz von digitalen Medien in der Lehre. Kalonde und Mousa (2016) befragten Lehrerausbildende $(n=90)$ in den USA zu Faktoren, die bei der Auswahl der digitalen Medien ausschlaggebend sind, die sie Lehramtsstudierenden in ihren Kursen zu Unterrichtsmethoden näherbringen. Mehr als die Hälfte der Lehrerausbildenden beantwortete die offen gestellte Frage mit der Passung der Technologie zum Lerninhalt (57\%) und der Benutzerfreundlichkeit (57\%) als ausschlaggebende Faktoren. Seltener nannten die Befragten ihre eigene Vertrautheit mit der Technologie (43\%), das Vorhandensein und Zugang zur Technologie (34\%), sowie die Kosten des Erwerbs und der Nutzung der Technologie (13\%). Zudem wurden in der Studie mit zehn Lehrerausbildenden vertiefende Interviews geführt. In diesen kam u. a. zur Sprache, dass die Lehrerausbildenden vor allem deshalb motiviert waren, Technologien einzusetzen, weil sie beim Einsatz von digitalen Medien in der Lehre positive Effekte bei den Studierenden (z. B. höhere Teilnahme, positive Rückmeldungen) wahrnehmen konnten.

\subsection{Institutionelle Unterstützung für Lehrerausbildende}

Die mit der Digitalisierung verknüpften Herausforderungen können die berufliche Identität von Lehrerausbildenden beeinflussen (Avidov-Ungar \& ForkoshBaruch, 2018). Insofern benötigen Lehrerausbildende die Unterstützung durch ihre Institution. Die institutionelle Unterstützung wird in mehreren Studien als bedeutsamer Faktor für die Integration von digitalen Medien in der Lehre genannt.

So gilt die institutionelle Unterstützung in zwei in den USA durchgeführten Befragungen als signifikanter Einflussfaktor für TPACK (Nelson et al., 2019; Voithofer et al., 2019). Die Unterstützung durch die Institution führt zu einem höheren technischen Wissen, was wiederum die Integration von digitalen Medien in die Lehre fördert (Nelson et al., 2019). Auch bei Instefjord und Munthe (2017) konnten (allerdings schwache) signifikante Korrelationen zwischen der Unterstützung durch Führungskräfte sowie positivem Management des Austauschs an den jeweiligen Institutionen und der selbsteingeschätzten Kompetenz der Lehrerausbildenden festgestellt werden. Die interviewten Lehrerausbildenden in der Studie von Avidov- 
Ungar und Forkosh-Baruch (2018) betonten, dass sie nicht nur zu technischen und pädagogischen Fragestellungen Hilfe benötigen, sondern - beispielsweise aufgrund hoher Arbeitsbelastung und Angst vor Veränderungen - auch emotionale Unterstützung. Unterstützung erwarteten die Lehrerausbildenden in der Studie von Avidov-Ungar und Forkosh-Baruch (2018) allerdings nur zu einem Drittel von ihrer Institution bzw. dem Bildungssystem als Ganzem, aber zu zwei Dritteln von ihren Studierenden. Dies liegt daran, dass sie auf deren Motivation, Kooperation und Flexibilität angewiesen sind. Die Institutionen sollten zudem die Technologien bereitstellen, da sonst kein Einsatz digitaler Medien in der Lehre stattfinden kann: In der Studie von Kalonde und Mousa (2016) wurde von zwei Dritteln (67\%) der befragten Lehrerausbildenden nicht vorhandene Technologie bzw. fehlender Zugang als größte Barriere für den Einsatz digitaler Technologien in der Lehre erwähnt. Zudem nannten die Befragten die fehlende Zeit (33\%), eine nicht adäquate Infrastruktur (30\%) sowie fehlenden technischen Support (20\%) als Hürden. Knapp die Hälfte (47\%) der Lehrerausbildenden hinderte geringes Wissen und fehlende Weiterbildungsmöglichkeiten am Einsatz digitaler Medien. Im Folgenden werden zwei in Studien beschriebene Weiterbildungsmaßnahmen für Lehrerausbildende skizziert.

\subsubsection{Kollaboration in der Vorbereitung von Lehre: Teacher Educator Design Teams}

Becuwe et al. (2017) begleiteten eine Weiterbildungsmaßnahme, in der Lehrerausbildende aus vier Institutionen kollaborativ in Teams zusammenarbeiteten und gemeinsam in sogenannten Teacher Educator Design Teams digitales Kursmaterial für die Lehrerbildung entwarfen. Die Idee dieser Teams ist es, dass Lehrerausbildende ähnlich wie Lehrkräfte in Teacher Design Teams (Binkhorst, Handelzalts, Poortman \& van Joolingen, 2015; Voogt, Pieters \& Handelzalts, 2016) - ihre TPACKKompetenzen durch die praktische Anwendung in Form der gemeinsamen (Um-) Gestaltung von Kursen erweitern.

Becuwe et al. (2017) befragten verschiedene Akteure (Lehrerausbildende, Coaches, Personen aus der Programmkoordination und Forschende) aus diesen Teams mithilfe von Fragebögen in einer Delphi-Studie zu den Bedingungen für eine erfolgreiche Implementation von Teacher Educator Design Teams. Sie identifizierten dadurch zehn Erfolgsbedingungen: Auf der Ebene der Teams wurde 1) eine Aufgabenstellung von langfristigem Wert, die 2) sowohl technologische, pädagogische als auch inhaltliche Aspekte umfasst, als Erfolgsfaktor benannt. Außerdem wurden Teammitglieder, die sich 3) gegenseitig vertrauen, 4) Feedback akzeptieren und 5) aufgeschlossen gegenüber Innovationen sind als Erfolgsindikatoren identifiziert. Hinzu kommt 6) eine durch Verantwortungsbewusstsein bei jedem Einzelnen gekennzeichnete Zusammenarbeit. Auch 7) einen flexiblen Coach, der 
auf das Team und seine Bedürfnisse eingeht, empfanden die Befragten als wichtig. Auf institutioneller Ebene ist laut den Befragten vor allem 8) die Unterstützung der Hochschulleitung von Bedeutung. Als zentrale strukturelle Rahmenbedingungen kristallisierten sich 9) ausreichend Zeit für die Mitglieder der Teacher Education Design Teams und 10) die Rolle der Coaches heraus.

\subsubsection{Kollaboration von Mentorinnen und Mentoren mit angehenden Lehrkräften}

Das von Liu et al. (2015) beschriebene Programm ist ein Beispiel, wie neben den angehenden Lehrkräften auch die betreuenden Lehrkräfte von Mentoring-Partnerschaften in Praktika profitieren können. Ziel des Programms ist es, dass statt der sonst eher asymmetrischen Beziehung zwischen einer betreuenden Lehrkraft und deren Mentees in der Praxisphase ein gegenseitiger Kompetenzerwerb auf Augenhöhe ermöglicht wird. In dem Programm führten eine angehende und eine berufstätige Lehrkraft abwechselnd jeweils zwei Unterrichtsstunden unter Nutzung von digitalen Medien durch. In der Studie von Liu et al. (2015) wurden die Stunden aufgezeichnet und in anschließenden Fokusgruppeninterviews reflektiert. Dabei profitierten die Mentorinnen und Mentoren vor allem von den technischen Kompetenzen der angehenden Lehrkräfte, während diese hauptsächlich aus der pädagogischen Erfahrung der Mentorinnen und Mentoren Nutzen ziehen konnten. Allerdings besteht auch die Gefahr, dass die technischen Aspekte zu sehr in den Vordergrund rücken. So berichtete eine befragte Lehrkraft davon, dass sich die Kollaboration der Mentorinnen und Mentoren untereinander auf das Zusenden digitaler Materialien beschränkte, sie sich aber nicht zu fachspezifischen oder pädagogischen Themen austauschten.

\section{Diskussion}

In der Forschung zu Digitalisierung in der Lehrerbildung sind Lehrerausbildende angesichts ihrer hohen Bedeutung als Vorbilder für angehende Lehrkräfte eine überraschend selten in den Blick genommene Zielgruppe (Starkey, 2019). Durch das Review konnte ein Einblick in internationale Studien zu diesen Akteuren gewonnen werden. Trotz einiger Einschränkungen und möglicher Verzerrungen im Auswahlprozess lassen sich aus den Ergebnissen sowohl für die Forschung als auch für Politik und Praxis Schlussfolgerungen ziehen. 


\subsection{Zusammenfassung}

Im Fokus dieses Reviews standen die für das Lehren und Lernen mit digitalen Medien benötigten Kompetenzen von Lehrerausbildenden, individuelle Faktoren, die den Einsatz digitaler Medien in der Lehre begünstigen sowie Möglichkeiten, wie Lehrerausbildende beim Kompetenzerwerb für den Einsatz digitaler Medien unterstützt werden können. Zur Beantwortung dieser Fragen wurde ein systematischer Überblick über den aktuellen Forschungsstand (2015-2019) gegeben.

Um angehende Lehrkräfte auf das Unterrichten mit digitalen Medien vorzubereiten, benötigen Lehrerausbildende sowohl pädagogische als auch technische Kompetenzen (Uerz et al., 2018). Durch das Review wurden mit TPACK, den SQD-Strategien sowie dem TETCs-Modell drei Modelle zur Operationalisierung von Wissen bzw. Kompetenz im Bereich Digitalisierung identifiziert. Das ursprünglich für Lehrkräfte konzipierte TPACK-Modell (Mishra \& Koehler, 2006) nimmt auch in der Forschung zu Lehrerausbildenden eine wichtige Rolle ein. Das Forschungsinteresse gilt in den durch das Review identifizierten Studien vorrangig den Einflussfaktoren auf das technologiebezogene fachdidaktische Wissen (TPACK) und wird nur selten im Zusammenhang mit den Kompetenzen angehender Lehrkräfte erhoben. Dagegen konnte in mehreren Studien ein positiver Zusammenhang zwischen von den Lehrerausbildenden eingesetzten SQD-Strategien und den Kompetenzen angehender Lehrkräfte zum Unterrichten mit digitalen Medien gefunden werden. Mit den auf Grundlage der Auswertung von qualitativen Studien identifizierten SQD-Strategien (Tondeur et al., 2012) werden pädagogisch-didaktische Handlungskompetenzen von Lehrerausbildenden beschrieben, die zur Vorbereitung angehender Lehrkräfte auf das Unterrichten mit digitalen Medien notwendig sind. Mit einer Ausnahme (Tondeur et al., 2019) wurden SQDStrategien in den aufgenommenen Studien durch Befragungen von Studierenden erhoben. Das TETCs-Modell (Foulger et al., 2017) leistet ebenfalls einen Beitrag zur Modellierung der Kompetenzen von Lehrerausbildenden. Das Modell beschreibt ein breites Spektrum an Kompetenzen und messbaren Indikatoren. Allerdings steht die Forschung zu den TETCs noch am Anfang.

Im Vergleich zu Kompetenzen der Lehrerausbildenden werden Einstellungen und Überzeugungen von Lehrerausbildenden selten in Studien thematisiert. Förderlich für den Einsatz digitaler Medien in der Lehre könnte beispielsweise eine konstruktivistische Sicht auf die Lehre sein (Taimalu \& Luik, 2019). Die vorgefundene Zweiteilung der Lehrerausbildenden in eine Personengruppe mit ausgeprägten digitalen Kompetenzen und positiven Einstellungen zu digitalen Medien und eine Gruppe mit geringeren Kompetenzen und negativen Einstellungen in der Studie von Tondeur et al. (2019) ist kohärent zu methodisch ähnlich angelegten Untersuchungen bei Lehrkräften und Lehramtsstudierenden (Drossel \& Eickelmann, 2018; Tondeur et al., 2017). Eine große Herausforderung für die Zukunft der Lehrerbildung ist die Frage, ob und vor allem wie Lehrerausbildende der letztgenannten Gruppe geför- 
dert werden können. Neben der Einstellung gibt es auch Hinweise darauf, dass das Vorhandensein von Technik und Infrastruktur (z. B. Kalonde \& Mousa, 2016) sowie das Wissen über die Bedienung von Soft- und Hardware bzw. über den Einsatz von digitalen Technologien in pädagogischen Kontexten (z. B. Taimalu \& Luik, 2019; Voithofer et al., 2019) wichtig für die erfolgreiche Integration von digitalen Medien in der Lehre sind. Es könnte sich also - ähnlich wie bei Lehrkräften (Knezek \& Christensen, 2019; Petko, 2012) - bei den Faktoren, die die Integration von digitalen Medien in die Lehre begünstigen, um ein Zusammenspiel aus einer positiven Einstellung, hohen Kompetenzen sowie einer ausreichenden technischen Ausstattung und Infrastruktur handeln.

In vielen Studien wurde deutlich, dass die Unterstützung durch die Institution eine wichtige Rolle bei der Implementierung digitaler Medien in die Lehre spielt. Die Unterstützung kann dabei sowohl inhaltlich, technisch als auch emotional sein. Eine mögliche Antwort auf die Frage, wie Lehrerausbildende sich untereinander in der Digitalisierung der Lehre unterstützen können, ist die Bildung von Teacher Educator Design Teams (Becuwe et al., 2017). Zudem ist die Förderung des Austauschs zwischen Lehrerausbildenden und angehenden Lehrkräften wünschenswert. Dieser Ansatz wurde in der Studie von Liu et al. (2015) verfolgt. Für die Praxis in der Lehrerbildung ergibt sich aus beiden vorgestellten Maßnahmen, dass Kommunikation und Kooperation wichtige Faktoren sind, um digitale Medien in die Ausbildung von angehenden Lehrkräften zu integrieren und diese auf das Unterrichten mit digitalen Medien vorzubereiten.

\subsection{Limitationen}

Auf Ebene der methodischen Durchführung des Reviews gibt es mögliche Einschränkungen. Es konnte durch die Literaturrecherche keine in Deutschland durchgeführte Studie zu Lehrerausbildenden identifiziert werden. Dies ist aufgrund der Besonderheiten der Lehrerbildung im Vergleich zu anderen Ländern z. B. Gliederung der Ausbildung in eine erste Phase an Hochschulen und eine zweite Phase im Vorbereitungsdienst (Blömeke, 2019) - kritisch zu sehen. Eine Ursache könnte an einer fehlerhaften Verschlagwortung in den Datenbanken (database bias, Booth et al., 2016, S. 203) oder der Wahl ungeeigneter Suchbegriffe liegen. Da die Suche jedoch breit angelegt war und mit der Kombination vieler unterschiedlicher Schlagwörter durchgeführt wurde, erscheint es wahrscheinlicher, dass im deutschsprachigen Raum Lehrerausbildende bisher in der Forschung zur Digitalisierung in der Lehrerbildung eine untergeordnete Rolle spielten. Durch die Verwendung von Indikatoren zur Einschätzung der Qualitätskriterien sollte ein objektives Verfahren zur Einschätzung der Studienqualität entwickelt werden. Aufgrund der Heterogenität der methodischen Zugänge ist eine Vergleichbarkeit der Kriterien und 
Indikatoren jedoch nicht immer gegeben. Zudem ist fraglich, ob alle Kriterien zur Qualitätsbewertung gleich gewichtet werden können.

Nicht auszuschließen ist, dass vor allem den Erwartungen entsprechende Studienergebnisse publiziert werden (publication bias; Newman \& Gough, 2020, S. 8). Die aufgenommenen Studien haben, wie in 3.1.2 beschrieben wurde, Schwächen. In den Studien wurden außerdem viele unterschiedliche Fragestellungen verfolgt. Deshalb gibt es nur wenige mehrfach abgesicherte Ergebnisse. Zum Teil sind die Befunde widersprüchlich, sodass weiterer Klärungsbedarf besteht.

\subsection{Schlussfolgerungen}

Aus den Ergebnissen dieses Reviews lassen sich sowohl für die Forschung als auch für die Praxis an Hochschulen und Ausbildungsseminaren Empfehlungen ableiten. Angesichts der sehr heterogenen Schwerpunktsetzungen der Studien zu Lehrerausbildenden sind Replikationsstudien bzw. vertiefende Studien wünschenswert. Zudem fehlt es an qualitativ hochwertigen Untersuchungen, in denen Mentorinnen und Mentoren in den Blick genommen werden. Dies ist insbesondere deshalb von Bedeutung, weil diese einen stärkeren Bezug zum Unterricht in der Schule als Lehrerausbildende an Hochschulen haben und deshalb hilfreiche Impulse für die Entwicklung der angehenden Lehrkräfte geben können. Mögliche Schwerpunktsetzungen für Forschungsvorhaben $\mathrm{zu}$ Lehrerausbildenden mit Relevanz für Praxis und Politik wären beispielsweise die Erstellung und Validierung von Instrumenten zur Messung von Kompetenzen und Einstellungen von Lehrerausbildenden im Bereich Digitalisierung, die Untersuchung von institutionellen Einflussfaktoren auf die digitalisierungsbezogenen Kompetenzen und Einstellungen von Lehrerausbildenden und die Entwicklung und Evaluation von Weiterbildungsmaßnahmen. Zur besseren Übertragbarkeit der Ergebnisse sollten im deutschsprachigen Raum Lehrerausbildende von der Forschung stärker in den Blick genommen werden.

Aus den international durchgeführten Studien lassen sich praxisrelevante Empfehlungen für die Optimierung der Lehrerbildung in Deutschland ableiten: Erstens wurde deutlich, dass Lehrerausbildende nicht nur technische, sondern auch pädagogische Kompetenzen bezüglich des Einsatzes digitaler Medien benötigen. Sie sollen Vorbilder für den Einsatz digitaler Medien sein und geeignete Strategien einsetzen, um angehende Lehrkräfte auf das Unterrichten mit digitalen Medien vorzubereiten. Um den Austausch über pädagogisch-didaktische Fragestellungen zu fördern, erscheint es wünschenswert, geeignete Formate $\mathrm{zu}$ entwickeln. Zweitens spielt die institutionelle Unterstützung eine wichtige Rolle für den Einsatz digitaler Medien in der Lehre. Für Hochschulen und Ausbildungsseminare bedeutet dies, dass sie die Lehrerausbildenden mit Technik und Infrastruktur ausstatten, ihnen aber auch ausreichend Zeit zur Vorbereitung und geeignete Weiterbildungs- 
und Austauschmöglichkeiten (Parrish \& Sadera, 2019) zur Verfügung stellen sollten. Drittens ist bei der institutionellen Unterstützung $\mathrm{zu}$ beachten, dass die Lehrerausbildenden sich stark unterscheiden, z. B. bezüglich Kompetenzen, Einstellung, Erfahrung oder der Fachzugehörigkeit. Insofern ist es eine große Herausforderung für Hochschulen und Politik, auf diese Heterogenität einzugehen. Möglicherweise ist eine strategische Ausrichtung der Hochschulen und Ausbildungsseminare an einem der vorgestellten Kompetenzmodelle hilfreich. Diese Maßnahmen könnten Lehrerausbildende beim Einsatz digitaler Medien in der Lehre unterstützen und die Qualität des Lehramtsstudiums und des Vorbereitungsdiensts verbessern.

\section{Literatur}

* durch Reviewprozess ausgewählte Studie

*Avidov-Ungar, O. \& Forkosh-Baruch, A. (2018). Professional identity of teacher educators in the digital era in light of demands of pedagogical innovation. Teaching and Teacher Education, 73, 183-191. https://doi.org/10.1016/j.tate.2018.03.017

${ }^{\star}$ Baran, E., Canbazoglu Bilici, S., Albayrak Sari, A. \& Tondeur, J. (2019). Investigating the Impact of Teacher Education Strategies on Preservice Teachers' TPACK. British Journal of Educational Technology, 50, 357-370. https://doi.org/10.1111/bjet.12565

${ }^{*}$ Becuwe, H., Roblin, N. P., Tondeur, J., Thys, J., Castelein, E. \& Voogt, J. (2017). Conditions for the Successful Implementation of Teacher Educator Design Teams for ICT Integration. A Delphi Study. Australasian Journal of Educational Technology, 33, 159172. https://doi.org/10.14742/ajet.2789

Binkhorst, F., Handelzalts, A., Poortman, C. L. \& van Joolingen, W. R. (2015). Understanding teacher design teams - A mixed methods approach to developing a descriptive framework. Teaching and Teacher Education, 51, 213-224. https://doi.org/10.1016/j. tate.2015.07.006

Blömeke, S. (2019). Lehrerbildung. In O. Köller, M. Hasselhorn, F. W. Hesse, K. Maaz, J. Schrader, H. Solga, C. K. Spieß \& K. Zimmer (Hrsg.), Das Bildungswesen in Deutschland. Bestand und Potenziale (S. 663-696). Bad Heilbrunn: Julius Klinkhardt.

Booth, A., Sutton, A. \& Papaioannou, D. (2016). Systematic approaches to a successful literature review. Los Angeles, CA: SAGE.

Brinkmann, B., Müller, U., Scholz, C. \& Siekmann, D. (2018). Lehramtsstudium in der digitalen Welt - Professionelle Vorbereitung auf den Unterricht mit digitalen Medien? Eine Sonderpublikation aus dem Projekt „Monitor Lehrerbildung“. Verfügbar unter https:// www.monitor-lehrerbildung.de/web/publikationen/digitalisierung/Ergebnisse-desMonitor-Lehrerbildung

${ }^{*}$ Cuhadar, C. (2018). Investigation of Pre-Service Teachers' Levels of Readiness to Technology Integration in Education. Contemporary Educational Technology, 9, 61-75. https:// doi.org/10.30935/cedtech/6211

Dillon, D., Chang, Y., Rondeau, A. \& Kim, J. (2019). Adopting TETCs in Integrated Elementary Mathematics and Technology Coursework: A Collaborative Self-Study of Two Teacher Educators. Journal of Technology and Teacher Education, 27(4), 527-554.

Drossel, K. \& Eickelmann, B. (2018). Die Rolle der Lehrerprofessionalisierung für die Implementierung neuer Technologien in den Unterricht - Eine Latent-Class-Analyse 
zur Identifikation von Lehrertypen. MedienPädagogik, 31, 166-191. https://doi. org/10.21240/mpaed/31/2018.06.04.X

Drossel, K., Eickelmann, B., Schaumburg, H. \& Labusch, A. (2019). Nutzung digitaler Medien und Prädiktoren aus der Perspektive der Lehrerinnen und Lehrer im internationalen Vergleich. In B. Eickelmann, W. Bos, J. Gerick, F. Goldhammer, H. Schaumburg, K. Schwippert, M. Senkbeil \& J. Vahrenhold (Hrsg.), ICILS 2018 \#Deutschland. Computer- und informationsbezogene Kompetenzen von Schülerinnen und Schülern im zweiten internationalen Vergleich und Kompetenzen im Bereich Computational Thinking (S. 205-240). Münster: Waxmann.

${ }^{*}$ Foulger, T. S., Graziano, K. J., Schmidt-Crawford, D. A. \& Slykhuis, D. A. (2017). Teacher Educator Technology Competencies. Journal of Technology and Teacher Education, 25, 413-448.

Gemeinsame Wissenschaftskonferenz. (2019). Pressemitteilung. „Qualitätsoffensive Lehrerbildung“" neue Förderrunde startet. Verfügbar unter https://www.gwk-bonn.de/filead $\mathrm{min} /$ Redaktion/Dokumente/Pressemitteilungen/pm2019-07.pdf

Giustini, D. (2019). Retrieving Grey Literature, Information, and Data in the Digital Age. In H. Cooper, L. V. Hedges \& J. C. Valentine (Hrsg.), The Handbook of Research Synthesis and Meta-Analysis (S. 101-126). New York, NY: SAGE. https://doi.org/10.7758/ 9781610448864.9

Goertz, L. \& Baeßler, B. (2018). Überblicksstudie zum Thema Digitalisierung in der Lehrerbildung. Arbeitspapier Nr. 36. Berlin: Hochschulforum Digitalisierung.

Grant, M. J. \& Booth, A. (2009). A typology of reviews: an analysis of 14 review types and associated methodologies. Health Information and Libraries Journal, 26, 91-108. https://doi.org/10.1111/j.1471-1842.2009.00848.x

${ }^{\star}$ Instefjord, E. J. \& Munthe, E. (2017). Educating digitally competent teachers. A study of integration of professional digital competence in teacher education. Teaching and Teacher Education, 67, 37-45. https://doi.org/10.1016/j.tate.2017.05.016

${ }^{\star}$ Kalonde, G. \& Mousa, R. (2016). Technology Familiarization to Preservice Teachers. Factors that Influence Teacher Educators' Technology Decisions. Journal of Educational Technology Systems, 45, 236-255. https://doi.org/10.1177/0047239515616965

Kay, R. H. (2006). Evaluating Strategies Used To Incorporate Technology Into Preservice Education: A Review Of the Literature. Journal of Research on Technology in Education, 38(4), 383-408. https://doi.org/10.1080/15391523.2006.10782466

Knezek, G. A. \& Christensen, R. (2016). Extending the will, skill, tool model of technology integration: adding pedagogy as a new model construct. Journal of Computing in Higher Education, 28, 307-325. https://doi.org/10.1007/s12528-016-9120-2

Knezek, G. A., Christensen, R. \& Furuta, T. (2019). Validation of a Teacher Educator Technology Competencies Survey. Journal of Technology and Teacher Education, 27(4), 465-498.

Kultusministerkonferenz. (2017). Bildung in der digitalen Welt. Strategie der Kultusministerkonferenz. Beschluss der Kultusministerkonferenz vom 08.12.2016 in der Fassung vom 07.12.2017. Verfügbar unter https://www.kmk.org/fileadmin/Dateien/pdf/PresseUnd Aktuelles/2018/Digitalstrategie_2017_mit_Weiterbildung.pdf

${ }^{\star}$ Liu, S.-H., Tsai, H.-C. \& Huang, Y.-T. (2015). Collaborative Professional Development of Mentor Teachers and Pre-Service Teachers in Relation to Technology Integration. Journal of Educational Technology \& Society, 18, 161-172.

Mishra, P. (2019). Considering Contextual Knowledge: The TPACK Diagram Gets an Upgrade. Journal of Digital Learning in Teacher Education, 35, 76-78. https://doi.org/ 10.1080/21532974.2019.1588611 
Mishra, P. \& Koehler, M. J. (2006). Technological pedagogical content knowledge: A framework for teacher knowledge. Teachers college record, 108, 1017-1054.

${ }^{*}$ Nelson, M. (2017). The Role of a Mentor Teacher's TPACK in Prospective Preservice Teachers' Intentions to Integrate Technology. Journal of Technology and Teacher Education, 25, 449-473.

${ }^{*}$ Nelson, M. J., Voithofer, R. \& Cheng, S.-L. (2019). Mediating factors that influence the technology integration practices of teacher educators. Computers \& Education, 128, 330-344. https://doi.org/10.1016/j.compedu.2018.09.023

Newman, M. \& Gough, D. (2020). Systematic Reviews in Educational Research: Methodology, Perspectives and Application. In O. Zawacki-Richter, M. Kerres, S. Bedenlier, M. Bond \& K. Buntins (Hrsg.), Systematic Reviews in Educational Research (S. 3-22). Wiesbaden: Springer VS.

Parrish, A. H. \& Sadera, W. A. (2019). A Review of Faculty Development Models that Build Teacher Educators' Technology Competencies. Journal of Technology and Teacher Education, 27(4), 437-464.

Petko, D. (2012). Teachers' pedagogical beliefs and their use of digital media in classrooms: Sharpening the focus of the 'will, skill, tool' model and integrating teachers' constructivist orientations. Computers \& Education, 58, 1351-1359. https://doi.org/10.1016/j. compedu.2011.12.013

Petko, D., Döbeli Honegger, B. \& Prasse, D. (2018). Digitale Transformation in Bildung und Schule: Facetten, Entwicklungslinien und Herausforderungen für die Lehrerinnen- und Lehrerbildung. Beiträge zur Lehrerinnen- und Lehrerbildung, 36, 157-174.

Rost, D. H. (2013). Interpretation und Bewertung pädagogisch-psychologischer Studien. Bad Heilbrunn: Klinkhardt. https://doi.org/10.1007/s35834-013-0069-1

Scheiter, K. (2017). Lernen mit digitalen Medien - Potenziale und Herausforderungen aus Sicht der Lehr-Lernforschung. Schulmanagement Handbuch, 164(4), 33-47.

Schmid, M. \& Petko, D. (2020). „Technological Pedagogical Content Knowledge“ als Leitmodell medienpädagogischer Kompetenz. In K. Rummler, I. Koppel, S. Aßmann, P. Bettinger \& K.D. Wolf (Hrsg.), Jahrbuch Medienpädagogik 17: Lernen mit und über Medien in einer digitalen Welt (S. 121-140). https://doi.org/10.21240/mpaed/jb17.X

Starkey, L. (2019). A review of research exploring teacher preparation for the digital age. Cambridge Journal of Education, 26(1), 1-20. https://doi.org/10.1080/030576 4X.2019.1625867

*Taimalu, M. \& Luik, P. (2019). The impact of beliefs and knowledge on the integration of technology among teacher educators. A path analysis. Teaching and Teacher Education, 79, 101-110. https://doi.org/10.1016/j.tate.2018.12.012

Thomas, A., Peterson, D. \& Abebe, F. (2019). Adopting TETCs in Integrated Elementary Mathematics and Technology Coursework: A Collaborative Self-Study of Two Teacher Educators. Journal of Technology and Teacher Education, 27(4), 499-525.

*Tondeur, J., Aesaert, K., Prestridge, S. \& Consuegra, E. (2018). A multilevel analysis of what matters in the training of pre-service teacher's ICT competencies. Computers \& Education, 122, 32-42. https://doi.org/10.1016/j.compedu.2018.03.002

${ }^{\star}$ Tondeur, J., Scherer, R., Baran, E., Siddiq, F., Valtonen, T. \& Sointu, E. (2019). Teacher Educators as Gatekeepers. Preparing the Next Generation of Teachers for Technology Integration in Education. British Journal of Educational Technology, 50, 1189-1209. https://doi.org/10.1111/bjet.12748

${ }^{*}$ Tondeur, J., Scherer, R., Siddiq, F. \& Baran, E. (2017). A Comprehensive Investigation of TPACK within Pre-Service Teachers' ICT Profiles. Mind the Gap! Australasian Journal of Educational Technology, 33, 46-60. https://doi.org/10.14742/ajet.3504 
Tondeur, J., van Braak, J., Sang, G., Voogt, J., Fisser, P. \& Ottenbreit-Leftwich, A. (2012). Preparing pre-service teachers to integrate technology in education: A synthesis of qualitative evidence. Computers \& Education, 59, 134-144. https://doi.org/10.1016/j. compedu.2011.10.009

Tondeur, J., van Braak, J., Siddiq, F. \& Scherer, R. (2016). Time for a new approach to prepare future teachers for educational technology use: Its meaning and measurement. Computers \& Education, 94, 134-150. https://doi.org/10.1016/j.compedu.2015.11.009

Uerz, D., Volman, M. \& Kral, M. (2018). Teacher educators' competences in fostering student teachers' proficiency in teaching and learning with technology: An overview of relevant research literature. Teaching and Teacher Education, 70, 12-23. https://doi. org/10.1016/j.tate.2017.11.005

Van Ackeren, I., Aufenanger, S., Eickelmann, B., Friedrich, S., Kammerl, R., Knopf, J., Mayrberger, K., Scheika, H., Scheiter, K. \& Schiefner Rohs, M. (2019). Digitalisierung in der Lehrerbildung. Herausforderungen, Entwicklungsfelder und Förderung von Gesamtkonzepten. DDS - Die Deutsche Schule, 111, 103-119. https://doi.org/10.31244/ dds.2019.01.10

*Voithofer, R., Nelson, M. J., Han, G. \& Caines, A. (2019). Factors that influence TPACK adoption by teacher educators in the US. Educational Technology Research and Development, 67, 1427-1453. https://doi.org/10.1007/s11423-019-09652-9

Voogt, J. M., Pieters, J. M. \& Handelzalts, A. (2016). Teacher collaboration in curriculum design teams: effects, mechanisms, and conditions. Educational Research and Evaluation, 22, 121-140. https://doi.org/10.1080/13803611.2016.1247725

Wang, W., Schmidt-Crawford, D. \& Jin, Y. (2018). Preservice Teachers' TPACK Development: A Review of Literature. Journal of Digital Learning in Teacher Education, 34, 234-258. https://doi.org/10.1080/21532974.2018.1498039 

Katharina Hähn \& Monique Ratermann-Busse

\title{
Digitale Medien in der Berufsbildung - Eine Herausforderung für Lehrkräfte und Ausbildungspersonal?
}

\begin{abstract}
Die berufliche Bildung übernimmt in Deutschland eine Schlüsselfunktion beim Übergang von der Schule in den Beruf. Aufgrund der Arbeitsmarktnähe sind die institutionellen Anpassungsanforderungen an die Berufsbildungspraxis, die durch eine digitalisierte Arbeitswelt gestellt werden, besonders hoch. Dies schließt die Entwicklung adäquater Medienkompetenzen des schulischen und betrieblichen pädagogischen Personals ein. In einem Critical Review wurden 14 Studien der Jahre 2013 bis 2019 identifiziert und nach festgelegten qualitativen Kriterien kodiert. Anschließend erfolgte eine systematische Aufarbeitung des Forschungstandes zu den Themenfeldern Anwendung digitaler Medien und Institutionalisierung digitaler Elemente in Bildungsinstitutionen sowie Kompetenzen, Haltung und Weiterbildung des pädagogischen Personals. Auf dieser Grundlage werden abschließend Forschungs- und Handlungsbedarfe aufgezeigt. Dies gilt insbesondere für die Entwicklung von Konzepten für die Weiterbildung des Berufsbildungspersonals sowie für den Nutzen, die Implementierung und den Bedarf digitaler Medien in der Berufsbildungspraxis.

Schlüsselwörter: Review, berufliche Bildung, Berufsbildung, Ausbildung, Digitalisierung, pädagogisches Personal, Medienkompetenz, medienpädagogische Kompetenzen, digitale Kompetenzen, Ausbildungspersonal, Ausbildungsleitungen, Lehrkräfte
\end{abstract}

\section{Digital media in vocational education and training - A challenge for teachers and VET staff?}

In Germany, vocational education and training (VET) take on a key role in the transition from school to work. Due to its proximity to the labour market, the institutional adaptation requirements for VET practice, which are necessary in a digitised world of work, are particularly high. Hence, teachers and company trainers need to develop suitable media-pedagogical competences. In a critical review, 14 studies were identified and coded covering the years 2013 to 2019, according to defined qualitative criteria that deal in different ways with the significance of digitisation processes in vocational education and training for educational staff. A review of the state of research then focuses on usability of digital media and institutionalisation of digital elements in educational institutions as well as competences, attitudes and further training of VET staff. Finally, research needs and requirements for action are outlined. These are particularly evident regarding the development of concepts for the further training of VET staff as well as the benefits, the implementation and the need of digital media in VET practices.

Keywords: review, VET, vocational training, vocational education, digitisation, pedagogical staff, media competence, digital competences, key competences, training staff, training managers, teachers, trainers 


\section{Einleitung}

Die Zunahme hochkomplexer Tätigkeitsfelder, hervorgerufen durch den Einsatz neuer Technologien, führt in nahezu allen Wirtschaftssektoren zu steigenden Kompetenz- und Qualifikationsanforderungen. Aufgrund hoher Digitalisierungsgrade müssen Berufsbilder und Qualifikationen an Entwicklungen wie Robotik, Additive Verfahren/3D-Druck, Web 2.0/Mobile Devices und Augmented Reality angepasst werden (Hirsch-Kreinsen, Ittermann \& Neuhaus, 2015). Dabei geraten die klassischen Bildungsinstitutionen der Berufsbildung zunehmend unter Druck, zukunftsfähige Qualifizierungsangebote zu schaffen.

„Wir stellen fest, dass die Digitalisierung auf allen Ebenen der beruflichen Bildung angekommen ist. Sie hat Auswirkungen auf nahezu alle Aus- und Fortbildungsberufe, sie verändert Aufgaben und verlangt neue Qualifikationen und Kompetenzen." (Präsident des BIBB Friedrich Esser)

Das Zitat zeigt, dass die Digitalisierung in der Berufsbildung zwei wesentliche Dimensionen beinhaltet. Zum einen steigt der Bedarf an entsprechend qualifizierten Fach- und Führungskräften sowohl mit fachspezifischen als auch fachübergreifenden (digitalen) Kompetenzen. Somit bedarf es einer „Berufsbildung für Digitalisierung“, bei der die Bedeutung einer Vermittlung von personalen und sozialen Kompetenzen sowie Medien- und Digitalisierungskompetenzen als Querschnittsaufgabe bei der Qualifizierung zunimmt. Zum anderen werden der Einsatz digitaler Medien sowie Digitalisierungsprozesse in der Bildungsarbeit zu zentralen Themen für die Bildungsorganisationsentwicklung. Hierbei geht es konkret um die „Digitalisierung für Berufsbildung". Sowohl für die Ausbildungsbetriebe, berufsbildende Schulen, Fachschulen, überbetriebliche Lehrwerkstätten und Kammern als institutionelle, kollektive und korporative Akteure der Berufsbildung auf der Mesoebene als auch für die Berufsschullehrkräfte, Ausbildungsverantwortlichen in Unternehmen und Prüfungsbeauftragten der Kammern als zentrale Akteure der Unterrichtsgestaltung auf der Mikroebene bergen diese Dimensionen besondere Herausforderungen (Kussau \& Brüsemeister, 2007, S. 31 ff.). Curricula bestehender Aus- und Fortbildungsberufe sowie Ausbildungsordnungen müssen den Bedarfen einer digitalisierten Arbeitswelt angepasst werden (BIBB, 2015). In einigen Bereichen werden inzwischen neue Ausbildungsberufe wie Kauffrau/-mann für E-Commerce für den Online-Einzelhandel geschaffen (BIBB, 2018). Zudem müssen digitale Lernmedien als Instrumente der Kompetenzvermittlung von den Institutionen der beruflichen Bildung in der eigenen Organisation implementiert und für die Unterrichtsgestaltung bereitgestellt werden. Gleichzeitig ist für ihren sinnvollen Einsatz im Unterrichtsgeschehen und in Unternehmen als weitere Lernorte der Berufsausbildung wichtig, dass das pädagogische Personal im Umgang mit digitalen Medien

1 siehe hierzu: https://www.bibb.de/de/pressemitteilung_109087.php 
qualifiziert ist und diese für die Wissens- und Kompetenzvermittlung angemessen nutzt, damit eine bedarfsgerechte Qualifizierung erfolgen kann, wie die KMK treffend formuliert:

„Dem didaktischen Prinzip der Praxisrelevanz folgend, müssen ferner künftige, durch die fortschreitende Digitalisierung ausgelöste Entwicklungen in der Arbeitswelt zeitnah in den Unterricht an beruflichen Schulen Eingang finden." (KMK, 2017)

Im Rahmen des Metavorhabens der aktuellen BMBF-Förderlinie „Digitalisierung im Bildungsbereich“ ist das vorliegende Dossier entstanden, welches einen umfassenden Überblick über den einschlägigen Forschungsstand zum des Einsatz digitaler Medien in der Berufsbildung und den damit verbundenen Herausforderungen für die Berufsschullehrkräfte und das Ausbildungspersonal geben soll. Dabei wird berücksichtigt, dass es sich bei der beruflichen Bildung um einen stark segmentierten Bildungsbereich mit unterschiedlichen institutionellen Verortungen (Ausbildungsvorbereitung, duale Berufsausbildung, schulische Berufsausbildung, berufliche Fortbildung) handelt. Als zentrale (Bildungs-)Institutionen auf der Mesoebene sind neben den berufsbildenden Schulen, Fachschulen und überbetrieblichen Ausbildungs- und Lehrwerkstätten auch die Ausbildungsbetriebe als Lernorte sowie die Kammern als zuständige Stellen nach dem Berufsbildungsgesetz (BBiG) zu nennen.

Bei der Aufbereitung des Forschungsstands zu den Herausforderungen der Digitalisierung für das Lehr- und Ausbildungspersonal ist aufgrund der besonderen Nähe zum Beschäftigungssystem daher zusätzlich zu Studien, die sich auf Berufsschul- und Fachschullehrkräfte beziehen, Forschungsliteratur relevant, bei der Ausbildungsverantwortliche und Mentoren in Unternehmen oder Prüfungsbeauftragte der Kammern in den Fokus der Untersuchungen gerückt werden. Schwerpunktmäßig setzt sich das vorliegende Dossier mit der Bedeutung der Digitalisierung für das Lehr- und Ausbildungspersonal in der beruflichen Erstausbildung auseinander. Dabei wird insbesondere auf den Bereich der dualen Berufsausbildung näher eingegangen, da die Sichtung der Publikationen zeigte, dass keine Studien existieren, die sich ganz explizit auf die Bereiche Ausbildungsvorbereitung, schulische Berufsausbildung oder beruflichen Fortbildung beziehen. ${ }^{2}$ Inhaltlich kristallisieren sich fünf thematische Schwerpunkte heraus, die in den Studien mit Blick auf das Lehr- und Ausbildungspersonal bearbeitet wurden:

2 Die Bereiche betriebliche und individuelle-berufsbezogene Weiterbildung beispielsweise sind im Rahmen des BMBF-Metavorhabens „Digitalisierung im Bildungsbereich“ dem Sektor Erwachsenenbildung (Kap 6) zugeordnet. 
1. Didaktisch-methodische Perspektive: Einsatzfelder und -möglichkeiten digitaler Medien in der Berufsbildungspraxis

2. Persönliche Haltung von Lehrkräften und vom Ausbildungspersonal

3. Kompetenzen des pädagogischen Personals

4. Weiterbildungsbedarf, -angebot und -nachfrage

5. Institutionelle Verankerung von digitalen Lernanwendungen und Medientechnik

\section{Methodisches Vorgehen}

Das Literaturauswahlverfahren war angelehnt an die Durchführung von Critical Reviews (Grant \& Booth, 2009). Der Rechercheprozess kann im einleitenden Kapitel von Wilmers et al. in diesem Sammelband nachvollzogen werden. Die erste Trefferliste für den Sektor der beruflichen Bildung umfasst 1.632 Publikationen, die zwischen 2010 und 2019 veröffentlicht wurden. ${ }^{3}$ Eine erste Eingrenzung erfolgte durch die Betrachtung des Publikationszeitraumes 2016 bis 2019, wodurch sich die Anzahl der Publikationen auf 664 reduzierte. Diese wurden in einem ersten Screening nach Passung zum Bildungssektor und Passung zum Thema des Dossiers hin selektiert, was auf 113 Veröffentlichungen zutraf. Ein großer Anteil der thematisch unpassenden Publikationen behandeln Untersuchungen zu Medienkompetenzen von Schülerinnen und Schülern oder Auszubildenden. Literatur die als thematisch passend kodiert wurde, jedoch Forschungsarbeiten in anderen Länderkontexten behandelt, wurde dahingehend geprüft, ob eine Übertragbarkeit der Ergebnisse auf den deutschen Raum möglich ist. In den meisten Fällen gelang dies aufgrund der schwierigen internationalen Vergleichbarkeit der unterschiedlichen Berufsbildungssysteme nicht, sodass diese Publikationen nicht verwendet werden konnten. Gleichzeitig zeigten sich Schwierigkeiten diese internationalen Forschungsergebnisse klar von anderen Bildungsbereichen, insbesondere der allgemeinen Schulbildung oder Erwachsenenbildung, abzugrenzen. Entscheidend war auch der Dokumententyp für eine Aufnahme oder den Ausschluss von Publikationen. So wurden etwa Editorials, Interviews, Konferenzberichte, Erfahrungsberichte und Abschlussarbeiten nicht einbezogen. Vereinzelt sind Veröffentlichungen auch aus Qualitätsgründen aussortiert worden, z. B. weil Fallzahlen fehlten oder die Vorgehensweise nicht nachvollziehbar abgebildet wurde. Nachrecherchiert wurden hingegen Langfassungen von Studien (z.B. ausgewählte Ergebnisdarstellungen oder Projektberichte), die in kurzen Artikeln der Trefferliste erwähnt worden sind.

Über den gewählten Zeitraum hinaus wurden dem Dossier-Sample vier Studien aus den Jahren 2013 bis 2015 hinzugefügt, von denen ein Teil in den bereits ausgewählten Studien zitiert wurde. Andere wiesen interessante methodisch aufwen-

3 Alle Trefferlisten sind durch das DIPF online zugänglich gemacht worden (siehe Wilmers et al. in diesem Band). 
digere Ergebnisse auf. Insgesamt blieb die Anzahl einschlägiger Studien hinter den Erwartungen zurück. ${ }^{4}$ Weitere vier Publikationen im Sample sind nicht in der ursprünglichen Treffermenge enthalten, da sie erst nach Abschluss der Suche veröffentlicht wurden oder eine neuere Version als in den Datenbanken ersichtlich verfügbar war. Somit werden die Ergebnisse aus 14 Studien in dem vorliegenden Dossier näher beschrieben. Bei der Kodierung wurden 41 Studien aufgrund fehlender thematischer Passung, mangelnder Übertragbarkeit z. B. aufgrund länderspezifischer Unterschiede oder qualitativer Kriterien ausgeschlossen.

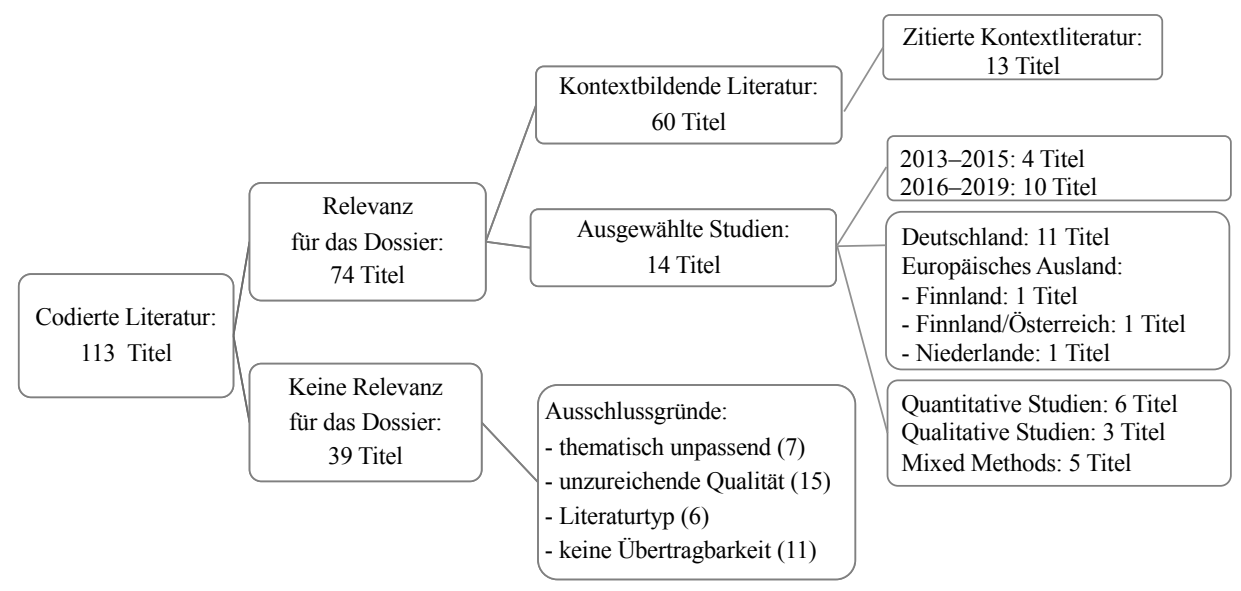

Abbildung 1: Auswahlprozess

\section{Beschreibung der verwendeten Studien}

Von den vierzehn ausgewählten Studien sind elf zum Teil oder ganz in Deutschland durchgeführt worden. Es wurden zur Bearbeitung der verschiedenen Fragestellungen sowohl quantitative (sechs Studien) als auch qualitative Methoden (drei Studien) sowie Mixed-Methods-Ansätze (fünf Studien) genutzt. Zwei Studien befassen sich dabei mit Instrumenten- und Modellentwicklungen zur Erfassung digitaler Kompetenzen des pädagogischen Personals.

In zwei Studien stand ausschließlich das betriebliche Ausbildungspersonal im Fokus der Befragung, wobei beide mit Unterstützung des Bundesinstituts für Berufsbildung (BIBB) herausgegeben wurden, was die Bedeutung des BIBB für Studien zum Ausbildungspersonal in der Forschungslandschaft widerspiegelt (Härtel et al., 2018b; BIBB, 2018). Sechs Studien beschäftigten sich mit Berufsschullehrkräften oder Berufsschulleitungen als Zielgruppen der Untersuchung (BMBF, 2018/2019; Conrad,

4 Auf die defizitäre Forschungslage, insbesondere für den betrieblichen Kontext, machen auch Härtelet al. (2018a) und Sloane et al. (2018) aufmerksam. Ein besonderes Forschungsdefizit scheinen überbetriebliche Berufsbildungsstätten (ÜBS) zu sein (z. B. Sloane et al., 2018). 
Wiest \& Schumann, 2015; IQSH, 2018; Kreijns, Vermeulen, Van Acker \& van Buuren, 2014; Seufert, Guggemos, Tarantini \& Schumann, 2019; Valtonen, Kontkanen, Dillon, Kukkonen \& Väisänen, 2013). Eine Publikation zu den Lernenden an beruflichen Schulen (Conrad \& Schumann, 2017) wird berücksichtigt, weil sie als Hauptstudie eines Arbeitsprogramms einer Vorstudie (Conrad et al., 2015) folgt, die wichtige Erkenntnisse zur persönlichen Haltung von Berufsschullehrkräften enthält. Vier Studien umfassen sowohl Daten von Berufsschullehrkräften als auch des Ausbildungspersonals (BIBB, 2014; Schmid, Goertz \& Behrens, 2016; Sloane et al., 2018; Virnes, Thiele, Manhart \& Thalmann, 2017). Eine Studie befragt sehr umfangreich Auszubildende und stellt einen Bezug zur Berufsschulsituation im Hinblick auf die Digitalisierung her (DGB, 2019). Die Datengrundlagen unterscheiden sich je nach methodischem Ansatz maßgeblich voneinander. Auffallend ist, dass die quantitativ angelegten Studien häufig äußerst geringe Fallzahlen aufweisen, was die Aussagekraft der Ergebnisse einschränkt (siehe hierzu Tabelle 1). Die meisten Studien werten die Daten auf der deskriptiven Ebene aus. Wenige Untersuchungen nutzen tiefergehende inferenzstatistische Verfahren wie Strukturgleichungsmodelle oder konfirmatorische Faktorenanalysen für die Auswertung und Interpretation der Ergebnisse (vgl. hierzu Kreijns et al., 2014; Seufert et al., 2018). Die thematischen Schwerpunkte persönliche Haltung von Lehrkräften und Ausbildungspersonal (sieben Studien) und didaktisch-methodische Perspektive: Einsatzfelder und -möglichkeiten digitaler Medien in der Berufsbildungspraxis (zehn Studien) standen am Häufigsten im Fokus des Interesses, gefolgt vom Themenschwerpunkt Kompetenz des pädagogischen Personals mit sechs Studien. Die Themenbereiche institutionelle Verankerung von digitalen Lernanwendungen und Medientechnik und Weiterbildungsbedarf, -angebot und -nachfrage werden nur in jeweils fünf Studien näher beleuchtet. Identifizierte Handlungs- und Forschungsbedarfe werden als Schlussfolgerungen im Fazit in den Mittelpunkt gerückt.

Insgesamt drei Studien stammen aus dem europäischen Ausland und konnten aufgrund ihrer strukturellen Nähe zum deutschen Berufsbildungssystem einbezogen werden. Eine quantitativ angelegte Studie bezieht sich auf das finnische Berufsbildungssystem (Valtonen et al., 2013). Eine qualitativ angelegte Studie geht auf eine Kooperation zwischen finnischen und österreichischen Wissenschaftlern und Wissenschaftlerinnen zurück (Virnes et al., 2017), wobei eine finnische und eine deutsche Fallstudie bearbeitet werden. Eine weitere methodisch interessante, quantitativ angelegt Studie wurde in den Niederlanden durchgeführt (Kreijns et al., 2014). 
Tabelle 1: Übersicht der ausgewählten Studien

\begin{tabular}{|c|c|c|c|c|c|c|c|c|c|c|c|}
\hline Autorenschaft & Jahr & 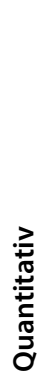 & 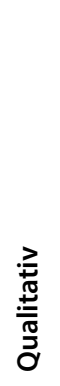 & 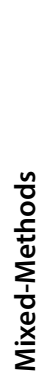 & $\mathrm{N}$ & 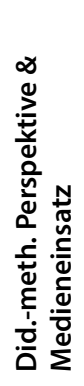 & 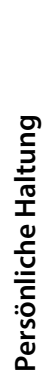 & 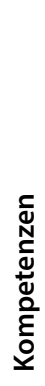 & 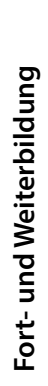 & 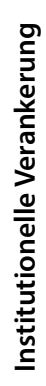 & 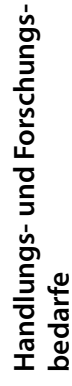 \\
\hline Valtonen et al. & 2013 & $\mathrm{x}$ & & & $241 ; 117$ & $\mathrm{X}$ & & & & & \\
\hline BIBB & 2014 & $\mathrm{X}$ & & & 376 & $\mathrm{X}$ & $\mathrm{x}$ & & $\mathrm{X}$ & $\mathrm{x}$ & $\mathrm{X}$ \\
\hline Kreijns et al. & 2014 & $\mathrm{x}$ & & & 158 & & $\mathrm{X}$ & $\mathrm{X}$ & & & \\
\hline Conrad et al. & 2015 & $\mathrm{X}$ & & & 42 & $\mathrm{X}$ & $\mathrm{X}$ & & & & \\
\hline Schmid et al. & 2016 & & & $\mathrm{x}$ & $303 ; 118$ & $\mathrm{x}$ & $\mathrm{x}$ & $\mathrm{X}$ & $\mathrm{x}$ & $\mathrm{X}$ & $\mathrm{X}$ \\
\hline Conrad et al. & 2017 & & & $\mathrm{x}$ & 103 & $x$ & & & & & \\
\hline Virnes et al. & 2017 & & $\mathrm{x}$ & & 37 & $\mathrm{x}$ & $x$ & & & & \\
\hline Jablonka et al. & 2018 & & $\mathrm{x}$ & & 77 & $\mathrm{x}$ & & & & & \\
\hline Härtel et al. & 2018 & & & $\mathrm{X}$ & $10 ; 733 ; 17$ & $\mathrm{x}$ & $\mathrm{x}$ & $\mathrm{X}$ & $\mathrm{X}$ & & $\mathrm{x}$ \\
\hline IQSH* & 2018 & $\mathrm{X}$ & & & 704 & & & & & $x$ & \\
\hline Sloane et al.* & 2018 & & $X$ & & 61 & & & $\mathrm{X}$ & $\mathrm{X}$ & $\mathrm{X}$ & $\mathrm{X}$ \\
\hline BMBF & $18 / 19$ & & & $\mathrm{X}$ & k. A. & $\mathrm{X}$ & & $\mathrm{X}$ & $\mathrm{X}$ & & \\
\hline DGB* & 2019 & $\mathrm{X}$ & & & 16.181 & $\mathrm{X}$ & & & & $\mathrm{X}$ & \\
\hline Seufert et al.* & 2019 & & & $\mathrm{X}$ & 215 & & & $\mathrm{X}$ & & & \\
\hline
\end{tabular}

*Nicht in ursprünglicher Trefferliste enthalten

\section{Ergebnisse}

Die Auswertung der Studien erfolgte entlang der fünf oben genannten Themencluster. Die einzelnen Schwerpunkte sind zum Teil stark miteinander verwoben oder bedingen sich gegenseitig. Zugunsten einer genauen Analyse des Forschungsstandes wurde die Abgrenzung der folgenden Dossierabschnitte voneinander dennoch trennscharf gehalten. 


\subsection{Didaktisch-methodische Perspektive: Einsatzfelder und -möglichkeiten digitaler Medien in der Berufsbildungspraxis}

Die technologischen Entwicklungen haben in den letzten Jahren zu einer starken Ausdifferenzierung des Angebots an digitalen Lernmedien (z. B. Augmented Reality, Simulationen, Social Networks, Blended Learning, Mobile Devices, Videos, Apps usw.) für die Wissens- und Kompetenzvermittlung geführt. Diese Entwicklung beinhaltet einen zunehmenden Stellenwert dieser Art des Lernens für die (Weiter) Entwicklung von Bildungsangeboten in der Berufsbildung im Allgemeinen und für die Bildungspraxis des Lehr- und Ausbildungspersonals im Besonderen (Vollmer et al., 2017). Dabei stellt sich die Frage, welche und inwiefern digitale Medien im berufsschulischen Unterricht bzw. am Lernort oder im praktischen Teil einer Ausbildung eingesetzt werden, wobei auch die infrastrukturellen Voraussetzungen an den Lernorten in Bezug auf Medientechnik, Hardware und Zugang zum Internet eine Rolle spielen. Zahlreiche Studien befassen sich ganz konkret mit dem Einsatz digitaler Lernanwendungen in der Bildungspraxis (Bach, 2016a, b; BIBB, 2014; BIBB, 2018; BMBF, 2019; Conrad et al., 2015; Conrad \& Schumann, 2017; DGB, 2019; Härtel et al., 2018b; Köhler \& Niethammer, 2018; Sloane et al., 2018; Hall et al., 2016; Scheer \& Wachter, 2016; Schmid et al., 2016). Im Fokus steht die Beschreibung der Medienart, der Nutzungshäufigkeit und -inhalte sowie die Einsatzfelder digitaler Medien in der Berufsbildung auf deskriptiver Ebene. Trotz der Vielfalt an Möglichkeiten zeigen die Ergebnisse studienübergreifend, dass digitale Lernanwendungen und -technologien vom Lehr- und Ausbildungspersonal in der Unterrichtspraxis überwiegend als Ergänzung zu traditionellen Formaten eingesetzt werden (Schmid et al., 2016, S. 13 ff.). Die Schwerpunkte liegen bei der Informationssuche im Internet in Erarbeitungs- und Strukturaufbauphasen und der Nutzung von MS-Office-Programmen (BIBB, 2014; BIBB, 2018; Conrad et al., 2015; DGB, 2019; Schmid et al., 2016).

Zwei deutsche Studien liefern zentrale Ergebnisse zu verwendeten digitalen Medienarten und ihren Einsatzfeldern in der Berufsausbildung sowie zur Ausstattung an Berufsschulen und Ausbildungsstätten in Bezug auf Medientechnik und Hardware. Zum einen ist hier die Studie von Schmid et al. (2016) zu nennen, die im Rahmen des Monitors Digitale Bildung ${ }^{5}$ für den Bereich der Berufsbildung erstellt wurde. Als Forschungsdesign wurde ein Mixed Method-Ansatz gewählt, bei dem neben der Durchführung von Fokusgruppengesprächen und leitfadengestützten Interviews eine umfangreiche Befragung von Ausbilderinnen und Ausbildern $(\mathrm{n}=200)$, Ausbildungsleitungen $(\mathrm{n}=50)$, Berufsschullehrpersonen $(\mathrm{n}=303)$, Berufsschulleitungen $(\mathrm{n}=118)$ und überbetrieblichen Bildungsstätten $(\mathrm{n}=42)$ er-

5 Der Monitor Digitale Bildung ist bildungssektorenübergreifend angelegt und befasst sich mit zentralen Fragestellungen zum digitalen Lernen. Siehe hierzu: https://www.bertelsmann-stiftung.de/ fileadmin/files/BSt/Publikationen/GrauePublikationen/Studie_Monitor-Digitale-Bildung_Berufliche-Ausbildung-im-digitalen-Zeitalter_IFT_2016.pdf (zuletzt aufgerufen am 27.01.2020) 
folgte. Damit stellt diese Studie die bisher umfangreichste Erhebung zu allen Themenschwerpunkten dieses Dossiers dar und fängt die Perspektiven verschiedener Akteure in der beruflichen Bildung zum digitalen Lernen ein (Schmid et al., 2016). Im Fokus des Interesses „stand die Frage nach dem Status quo des digitalen Lernens im Ausbildungswesen." (Schmid et al., 2016, S. 9)

Die zweite Studie stammt aus dem BIBB (2014) und hatte einerseits die Nutzung des Portals foraus.de ${ }^{6}$ sowie Implikationen für dessen Weiterentwicklung im Blick. Andererseits war ein weiteres Untersuchungsziel, aktuelle Erkenntnisse zum Einsatz von digitalen Medien in der dualen Berufsbildung zu erlangen (BIBB, 2014, S. 1). Als Expertinnen und Experten wurden Personen befragt, die dem Berufsbildungspersonal $(n=376)$ zuzuordnen sind. Darin enthalten sind die Antworten von nebenberuflichen Ausbilderinnen und Ausbildern (22\%), hauptberuflichen Ausbilderinnen und Ausbildern (18\%), Lehrkräften zur Qualifizierung des Bildungspersonals (17\%) und Berufsschullehrkräften (6\%). $12 \%$ der Befragten sind nicht direkt in der Ausbildung tätig.

Die konkreten Fragestellungen der beiden Studien sind zwar unterschiedlich, aber trotzdem liefern Vergleiche einzelner Aspekte Befunde darüber, welche Medien zum Einsatz kommen, wo die Einsatzfelder liegen und wie sich die Nutzungshäufigkeit darstellt.

Insgesamt bestätigt sich das Bild einer eingeschränkten und wenig differenzierten Nutzung digitaler Medien in der Ausbildung und im Unterricht. Somit wird das breite Repertoire an digitalen Lernanwendungen derzeit noch nicht ausgeschöpft. Lernmanagementsysteme sind zwar schon länger existent, aber in der Bildungsorganisation bisher eher wenig verbreitet. So finden Selbstlernprogramme, Apps, Simulationen oder das Game-based-Learning sowohl in Ausbildungsbetrieben als auch in berufsbildenden Schulen nur begrenzt Anwendung (Schmid et al., 2016, S. 27, S. 32). Sie werden nur wenig genutzt und nicht in die alltägliche Unterrichtspraxis integriert. Die Studie von Schmid et al. (2016) stellt ergänzend fest, dass die Möglichkeiten des Einsatzes digitaler Medien zur individuellen Förderung, zur Motivationssteigerung oder zur Unterstützung bei Lerndefiziten kaum Gebrauch finden (Schmid et al., 2016, S. 16). Zur Gestaltung problem- bzw. projektorientierter Unterrichtseinheiten oder zur Entwicklung eigener Lehrinhalte sowie für das kollaborative Lernen kommen sie insgesamt eher selten zum Einsatz, wie auch die finnische Studie von Valtonen et al. (2013) bestätigt. Kostenpflichtige Lernanwendungen werden häufiger von betrieblichem Ausbildungspersonal als von den Berufsschullehrkräften eingesetzt. Generell werden die Kosten für die Beschaffung und Wartung der Technik sowohl von Ausbilderinnen und Ausbildern als auch von Berufsschullehrkräften als besondere Herausforderung gesehen (Schmid et al., 2016, S. 21).

6 Portal für Ausbilder/innen. Siehe hierzu: https://www.foraus.de/de/index.php (zuletzt aufgerufen am 27.01.2020) 
Tabelle 2: Vergleichende Darstellung zweier exemplarischer Studien zum Einsatz digitaler Medien

\begin{tabular}{|c|c|c|}
\hline & $\begin{array}{l}\text { Schmid et al., } 2016 \\
\text { (Berufsschullehrkräfte; } n=303 \text { ) } \\
\text { Ausbilder/innen; } n=200 \text { ) }\end{array}$ & $\begin{array}{l}\text { BIBB, } 2014 \\
\text { (Berufsbildungspersonal; } \\
n=200-257 \text { ) }\end{array}$ \\
\hline \multirow[t]{3}{*}{$\begin{array}{l}\text { Digitale } \\
\text { Medien- } \\
\text { art }\end{array}$} & $\begin{array}{l}\text { - Software (z. B. Microsoft Office) setzen } \\
44 \% \text { häufig ein (S. 14). }\end{array}$ & $\begin{array}{l}\text { - Office-Programme nutzen } 86 \text { \% regel- } \\
\text { mäßig im Rahmen der Ausbildung } \\
\text { (S. 8). }\end{array}$ \\
\hline & $\begin{array}{l}\text { Selbstlernprogramme (z. B. Simulatio- } \\
\text { nen, Lernspiele, Lern-Apps) setzen } 5 \% \\
\text { der Berufsschullehrkräfte häufig ein } \\
\text { (S. 14) und } 8 \% \text { der Ausbilder/innen. }\end{array}$ & $\begin{array}{l}\text { - Lernprogramme nutzen } 26 \% \text { regelmä- } \\
\text { Big im Rahmen der Ausbildung. } \\
\text { - Lernplattformen werden von } 18 \% \\
\text { regelmäßig verwendet. }\end{array}$ \\
\hline & $\begin{array}{l}\text { - Lernmanagementsysteme (z. B. Moodle) } \\
\text { setzen } 12 \% \text { der Lehrkräfte ein. }\end{array}$ & $\begin{array}{l}\text { - Digitales Berichtsheft nutzen } 29 \% \\
\text { regelmäßig. }\end{array}$ \\
\hline $\begin{array}{l}\text { Einsatz- } \\
\text { felder }\end{array}$ & $\begin{array}{l}\text { - } 46 \% \text { der Ausbilder/innen nutzen häufig } \\
\text { das Internet für die Recherche von Inhal- } \\
\text { ten; } 38 \% \text { der Berufsschullehrkräfte nut- } \\
\text { zen es häufig zur Informationsrecherche. } \\
\text { - } 24 \% \text { der Lehrkräfte und } 25 \% \text { der Ausbil- } \\
\text { der/innen verwenden häufig Lernvideos } \\
\text { oder Präsentationstools (z. B. Lehrvorträ- } \\
\text { ge, Unterweisungen). }\end{array}$ & $\begin{array}{l}\text { - } 31 \% \text { setzen regelmäßig Informations- } \\
\text { angebote im Unterricht ein. } \\
\text { - } 16 \% \text { nutzen Web } 2.0 \text { (Wikis, Foren, } \\
\text { Blogs). } \\
\text { - } 13 \% \text { arbeiten regelmäßig Software zum } \\
\text { Testen und Prüfen. }\end{array}$ \\
\hline $\begin{array}{l}\text { Medien- } \\
\text { technik }\end{array}$ & $\begin{array}{l}\text { - } 66 \% \text { der Lehrkräfte und } 53 \% \text { der Aus- } \\
\text { bilder/innen nutzen einen PC der Schule } \\
\text { für den Unterricht. } \\
\text { - } 44 \% \text { der Lehrkräfte und } 13 \% \text { der Ausbil- } \\
\text { der/innen nutzen ein interaktives White- } \\
\text { board der Schule. }\end{array}$ & $\begin{array}{l}\text { - } 89 \text { \% nutzen regelmäßig einen PC. } \\
\text { - } 10 \text { \% setzen regelmäßig ein interaktives } \\
\text { Whiteboard ein. }\end{array}$ \\
\hline
\end{tabular}

Der Einsatz digitaler Formate (z. B. Mathe-Apps, Sprachlernprogramme), die sich auf das Allgemeinwissen beziehen, ist ebenfalls sehr gering ausgeprägt, was möglicherweise in der inhaltlichen Bezugnahme auf fachspezifische Lerninhalte in der beruflichen Bildung begründet liegt. Teilweise existieren unternehmens- oder berufsspezifische Lernanwendungen. Je nach Ausbildungsberuf gibt es Unterschiede bei der Nutzung verschiedener Anwendungen, was sicherlich mit der Bedeutung der Digitalisierung für die verschiedenen Berufsfelder und -gruppen zusammenhängt (DGB, 2019, S. 15). Während beispielsweise elektronische Tests eher in dem Fachbereich Naturwissenschaft/Informatik zum Einsatz kommen, werden VideoAngebote eher für die Wissens- und Kompetenzvermittlung in den Bereichen Gesundheit/Soziales und Bau/Architektur genutzt, wie der Monitor Digitale Bildung übergreifend zeigt (Schmid et al., 2016, S. 19).

Neuere Medientechnik/Hardware wie interaktive Whiteboards, Tablets oder Smartphones setzt das Lehr- und Ausbildungspersonal eher selten ein (Schmid et al., 2016, S. 31). Bei der Möglichkeit zur Nutzung solcher technischen Geräte lassen 
sich in Bezug auf die duale Ausbildung ebenfalls berufsfeldspezifische Unterschiede erkennen. In technikaffinen Ausbildungsberufen bzw. da, wo technische Geräte zum Arbeitsalltag gehören - wie beispielsweise in der Informatik, der Elektronik, der Mechatronik oder im kaufmännischen Bereich - werden diese digitalen Geräte tendenziell eher genutzt als in Bereichen wie der Gastronomie oder in personenbezogenen Dienstleistungen, in denen der Digitalisierung engere Grenzen gesetzt sind bzw. die geringere Digitalisierungsgrade aufweisen (DGB, 2019, S. 16; BIBB, 2018). Was die Ausstattung mit digitalen Geräten wie Whiteboards und Tablets betrifft, bestehen Unterschiede zwischen den berufsbildenden Schulen und den überbetrieblichen Bildungsstätten zu Gunsten der berufsbildenden Schulen (Schmid et al., 2016, S. 32).

Zwei Studien beschäftigen sich methodisch tiefergehend mit dem Einsatz digitaler Medien in der Unterrichtpraxis. Eine finnische quantitativ angelegte Studie von Valtonen et al. (2013), bei der Lehrkräfte der Sekundarstufe II $(\mathrm{n}=241)$ und Berufsschullehrkräfte $(\mathrm{n}=117)$ befragt wurden, beschäftigt sich unter Bezugnahme auf den "technolological pedagogical content knowledge framework (TPACK)" mit dem Einsatz von social software (z. B. Blogs, Wikis, google tools, video sharing, social games). Beim TPACK ${ }^{7}$ wird davon ausgegangen, dass Lehrkräfte verschiedene Wissensbestände (technologisches, pädagogisches und inhaltliches Wissen $\rightarrow$ pädagogisch-inhaltliches Wissen, technologisch-pädagogisches Wissen, technologischpädagogisches Inhaltswissen) benötigen, um Schülerinnen und Schülern durch den Einsatz von Technologien erfolgreich Lerninhalte zu vermitteln. Zwei zentrale Fragestellungen wurden in der Studie von Valtonen et al. (2013) bearbeitet: Welche Social Software nutzen Lehrkräfte in ihrer Freizeit? Welcher Zusammenhang besteht zwischen der Nutzung von Social Software in der Freizeit und ihrer Anwendung in der Unterrichtspraxis? Vertiefend zeigen die Ergebnisse, dass finnische Berufsschullehrkräfte, die Social Software (z.B. Blogs, Wikis, Microblogs, Videosharing, sozialen Netzwerken usw.) in ihrer Freizeit nutzen, diese auch häufiger im Unterricht einsetzen. Allerdings sind Social Software-Anwendungen und ihr Potenzial sowie die Nutzungsmöglichkeiten zur Unterstützung des kollaborativen Lernens im Technologieverständnis von Lehrkräften im Erhebungszeitraum der Studie noch nicht so stark verankert. Überwiegend werden sie zur Veranschaulichung bestimmter Inhalte genutzt, weniger zu Kommunikationszwecken oder zur Gestaltung von Inhalten (Valtonen et al., 2013, S. $773 \mathrm{ff}$.).

Eine qualitativ angelegte Studie geht auf eine Kooperation zwischen finnischen und österreichischen Wissenschaftlerinnen und Wissenschaftlern zurück (Virnes et al., 2017). Die Ergebnisse basieren auf zwei Fallstudien, wobei eine in Finnland und eine in Deutschland durchgeführt wurde. Sie liefern Forschungsergebnisse sowohl zur persönlichen Haltung von Auszubildenden und dem Lehr- und Ausbildungspersonal sowie zum generellen Einsatz digitaler Lernmedien. Es handelt sich hierbei um ein Pilotprojekt, bei dem die Lern-App „Ach So!“ zum unterstützenden Lernen für Auszubildende im Konstruktionsbereich in Finnland und Deutschland

7 https://www.gfdb.de/didaktik-tpack-modell/ 
implementiert wurde. Zentrale Forschungsfragen waren hierbei, wie mobile Endgeräte und Apps zum Lernen am Arbeitsplatz genutzt werden und welchen Einfluss sie auf das Lernen haben. An den Fallstudien haben sowohl Auszubildende und Berufsschullehrkräfte als auch betriebliches Ausbildungspersonal und Unternehmensvertreterinnen und -vertreter $(\mathrm{n}=50)$ teilgenommen. Die Ergebnisse der Studie von Virnes et al. (2017) zum Einsatz eines bestimmten mobilen Lernprogramms zeigen, dass mobile Endgeräte viele Möglichkeiten für ein technologieerweitertes Lernen bieten und gezielt zur Unterstützung der Ausbildung eingesetzt werden können. Dadurch werden ein verbesserter Austausch sowie die Reflexion kritischer Arbeitssituationen zwischen Auszubildenden und Lehrkräften ermöglicht. Zudem kommt es zu einer flexibleren Erreichbarkeit innerhalb der Lehr-LernArrangements in der Ausbildung. Eine individuellere Betreuung bietet in diesem Zusammenhang Potenzial für die Förderung von Auszubildenden mit Lerndefiziten und die Herstellung eines Bezugs zu konkreten Lernbedarfen der Auszubildenden.

Ein Aspekt, der nur von Sloane et al. (2018) explizit einbezogen wird, ist der Nutzenaspekt digitaler Medien. Die Autorinnen und Autoren betonen mehrfach, dass der Einsatz digitaler Medien als Handwerkszeug zur Integration von Digitalisierung im Berufsbild zu verstehen ist und nicht einem Selbstzweck dienen soll:

„Für die Rolle digitaler Medien im Unterricht und in der betrieblichen Unterweisung ergibt sich daher grob ein Spannungsfeld zwischen plötzlich verfügbaren innovativen digitalen Technologien, die pädagogisch nicht sinnvoll eingesetzt werden bzw. alleine keine Verbesserung der Bildungsarbeit mit sich bringen, z.B. die Einführung von Smartboards ohne entsprechende Fortbildung der Lehr- und Ausbildungskräfte auf der einen Seite. Auf der anderen Seite stehen technische Potentiale zur Realisierung von pädagogisch Wünschenswertem (z.B. verstärkte Aktivierung der Lernenden, Individualisierung von Lernwegen und kompetente Technologienutzung), die ungenutzt bleiben.“ (Sloane et al., 2018, S. 122)

\subsection{Zur persönlichen Haltung von Lehrkräften und Ausbildungspersonal}

Studien, die sich auf den Themenschwerpunkt persönliche Haltung beziehen, setzen sich damit auseinander, welche Einstellungen und Verhaltensweisen das Lehrund Ausbildungspersonal bei der Nutzung digitaler Medien zeigt. Sieben Studien aus dem Bestand der untersuchten Literatur beziehen sich auf den Themenschwerpunkt persönliche Haltung.

Vier Studien zeigen, dass eine positive Einstellung und Überzeugungen sowie die Eigeninitiative von Lehrkräften und betrieblichem Ausbildungspersonal den Einsatz digitaler Medien begünstigen (Conrad et al., 2015; Härtel et al., 2018b; Kreijns et al., 2014; Schmid et al., 2016). Die Grundhaltungen gegenüber digitalen Medien sind beim pädagogischen Personal sowohl im Hinblick auf den Nutzen und das Potenzial für die Unterrichtsgestaltung als auch hinsichtlich positiver 
Effekte für Lernergebnisse oder ihrer entlastenden Funktion im berufsschulischen Alltag eher ambivalent ausgeprägt, wie die Studie von Schmid et al. (2016) zeigt: „Nur ein Drittel der befragten Berufsschullehrer sieht im Lernen mit Computern die Möglichkeit zur Verbesserung der Lernergebnisse (33 Prozent) oder eine Entlastung für ihre Arbeit (28 Prozent)" (Schmid et al., 2016, S. 24). Dahingegen verdeutlicht eine Studie des BIBB (2014), dass das Berufsbildungspersonal eine positive Grundhaltung gegenüber der entlastenden Funktion von digitalen Medien hat. Hier stimmten sogar $85 \%$ der Befragten eher oder vollkommen zu, dass der Einsatz digitaler Medien den Arbeitsalltag erleichtert (BIBB, 2014, S. 10). Eine Steigerung der Peer-to-Peer-Interaktion sowie einen besseren Austausch zwischen Ausbildern und Ausbilderinnen und Auszubildenden ermöglicht beispielsweise der Einsatz von mobilen Geräten (Virnes et al., 2017). In einigen Studien schwingt eine gewisse Skepsis gegenüber der Nutzung digitaler Medien mit (Härtel et al., 2018b; Schmid et al., 2016; Sloane et al., 2018; Virnes et al., 2017). Gefahren sowohl auf Seiten der Lehrkräfte als auch auf Seiten des Ausbildungspersonals werden insbesondere bei der Nutzung digitaler Medien zur Lernkontrolle sowie zur Lernleistung, zum Verständnis des Unterrichtsstoffs und zur Vermittlung schulischer Grundfertigkeiten gesehen (BIBB, 2014; Conrad et al., 2015; Härtel et al., 2018b; Virnes et al., 2017). 70\% des betrieblichen Ausbildungspersonals gehen davon aus, dass die Grundfertigkeiten unter der Verbreitung digitaler Medien leiden (Härtel et al., 2018b, S. 29).

Insgesamt erkennen Lehrkräfte sowie Ausbilderinnen und Ausbilder den zunehmenden Stellenwert von digitalen Medien in der Bildungsarbeit und sehen eine Verdrängung analoger Medien. Berufsschulleitungen haben die strategische Bedeutung des digitalen Lernens für die Schul- und Unterrichtsentwicklung noch nicht im Blick (BIBB, 2014; Härtel et al., 2018b; Schmid et al., 2016). Trotzdem sind die Berufsschulleitungen grundsätzlich offener für digitale Lernformen als Ausbildungsleitungen und Leitungen überbetrieblicher Einrichtungen (Schmid et al., 2016). Die Potenziale zur Verbesserung des Theorie-Praxis-Transfers und der Verzahnung zwischen den Lernorten Betrieb und Berufsschule werden insgesamt noch nicht ausreichend erkannt. So stimmen nur 30\% der Berufsschullehrkräfte voll und ganz zu, dass die Zusammenarbeit mit Ausbildungsbetrieben und dem betrieblichen Ausbildungspersonal durch die Digitalisierung einfacher wird. Nur $21 \%$ der Ausbilderinnen und Ausbilder stimmen voll und ganz zu, dass die Zusammenarbeit mit Berufsschullehrkräften einfacher wird (Schmid et al., 2016, S. 28).

Es scheint ein Zusammenhang zwischen der Haltung gegenüber digitalen Medien und soziodemografischen Merkmalen zu bestehen (Schmid et al., 2016, S. 25; Seufert et al., 2019). Neigungen zum Einsatz digitaler Medien und kritische Einstellungen lassen sich an demografischen Merkmalen festmachen, z.B. Geschlechter- oder Altersabhängigkeit. So zeigt beispielsweise eine Clusteranalyse aus der Studie von Schmid et al. (2016), dass die Kritikerinnen und Kritiker bei den Berufsschullehrkräften überwiegend 60 Jahre und älter sind und eine 
Berufserfahrung von 21 Jahren und mehr haben. Außerdem stehen Frauen der Nutzung von digitalen Lernanwendungen kritischer gegenüber. Lehrkräfte, die den Einsatz digitaler Medien befürworten, sind überwiegend Männer zwischen 30 und 39 Jahren und Männer mit einer relativ geringen Berufserfahrung von 1 bis 5 Jahren.

\section{Zusammenhang zwischen persönlicher Haltung und Medieneinsatz - Relevante Faktoren für den Einsatz digitaler Medien}

Zwei Studien nutzen als Grundlage zur Erklärung von Verhaltensweisen und Einstellungen - als wesentliche Merkmale der persönlichen Haltung - beim Einsatz digitaler Medien verschiedene theoretische Modelle (Conrad et al., 2015; Kreijns et al., 2014; Valtonen et al., 2013). Mit Hilfe des Technologieakzeptanzmodells von Venkatesh und Davis (2000) und einem Ansatz aus der Akzeptanzforschung von Müller-Böling und Müller (1986), bei dem Benutzertypen festgelegt wurden, wird in einer deutschen Studie von Conrad et al. (2015) geschaut, welche Faktoren die Akzeptanz von digitalen Lernmedien bedingen (Conrad et al., 2015). Dabei wird zwischen der Einstellungs- und Verhaltensakzeptanz unterschieden, wobei sowohl die Einstellung in Bezug auf das Verhalten als auch das beobachtete Verhalten selbst relevant sind (Conrad et al., 2015, S. 254). Der Fokus liegt hierbei auf den für die Nutzungseinstellung wesentlichen Faktoren „wahrgenommener Nutzen“ und „wahrgenommene einfache Bedienbarkeit" von Bildungstechnologien (Conrad et al., 2015, S. 255). Die grundlegende These ist: „Je größer diese beiden Faktoren ausgeprägt sind, desto eher wird der Benutzer/die Benutzerin die jeweilige Technologie auch tatsächlich einsetzen“ (Conrad et al., 2015a, S. 255). Mit Bezug zum webbasierten informellen Lernen im Wirtschaftsunterricht verdeutlichen zentrale Ergebnisse der Studie, dass ein positiver Zusammenhang zwischen der Nutzungseinstellung der Lehrkräfte und der Häufigkeit der Internetnutzung im Unterricht besteht, sodass die These des Technologieakzeptanzmodells untermauert wird. Mangelnde Fähigkeiten und Kenntnisse sowie die Verfügbarkeit von Medientechniken wie PCs hängen dagegen nicht mit dem Medieneinsatz zusammen (Conrad et al., 2015, S. 257). Als übergeordnetes Unterrichtsziel für den webbasierten Technologieeinsatz im Unterricht der befragten Lehrkräfte kristallisiert sich die Förderung fachlicher Kompetenzen heraus. Positive Effekte werden zudem im Hinblick auf die Lernmotivation, die Lernzufriedenheit und das Unterrichtsinteresses gesehen. Allerdings werden auch negative Effekte in Bezug auf das Verständnis „der allgemeinen Lernleistung, des Unterrichtsstoffs sowie hinsichtlich der Transferleistung" (Conrad et al., 2015, S. 258) befürchtet, was eine kritische Haltung der Lehrkräfte aufzeigt. ${ }^{8}$

Die niederländische Studie von Kreijns et al. (2014) bezieht sich auf eine größere Datengrundlage, lässt aber keine differenzierte Darstellung der Ergebnisse

8 Die Ergebnisse beruhen auf einer sehr geringen Fallzahl. Daher ist nur eine begrenzte Aussagekraft gegeben. Allerdings ist es eine der wenigen Studien, die sich theoriebasiert mit der persönlichen Haltung beschäftigen und die einzige, die das Technologieakzeptanzmodell zugrunde legt. Aus diesem Grund wurde sie in das Studiensample aufgenommen. 
für die Zielgruppe der Berufsschullehrkräfte zu. Die Autoren und Autorinnen haben ihre Untersuchung auf Basis einer Kombination der drei theoretischen Modelle self determination model (SDT), integrative model of behaviour predicition (IMBP) und Vallerand's hierarchical model of motivation durchgeführt. Die Studie befasste sich mit der Frage, ob die Modelle immer noch gültig sind, wenn sie als kombiniertes Modell für die Erfassung der Verwendung von digitalen Lernmaterialien (DLM) durch Lehrer und Lehrerinnen angewendet werden. Zentral ist hierbei herauszufinden, in welchem Zusammenhang Einstellungen und Motivationen sowie eine hohe Selbstwirksamkeitserwartung, Haltungen und erfüllte Grundbedürfnisse mit der Nutzung von digitalen Lernmaterialien stehen. Die Resultate eines Strukturgleichungsmodells zeigen, dass das kombinierte Modell valide ist und die erhobenen Faktoren somit eine Aussagekraft aufweisen (Kreijns et al., 2014, S. 474). Das kombinierte Modell liefert eine umfassendere Erklärung des willentlichen Verhaltens von Lehrerinnen und Lehrern bei der Verwendung von digitalen Lernmaterialien in ihrer pädagogischen Praxis, als die Verwendung der Modelle SDT und IMBP. Daran anschließend wurde der Frage nachgegangen, welche Implikationen das kombinierte Modell in Bezug eine mögliche Förderung der Lehrkräfte bei der Nutzung von digitalen Lernmaterialien mit sich bringt. Die Ergebnisse verdeutlichen, dass eine positive Selbsteinschätzung der Lehrkräfte zu ihrer Anwendungskompetenz bei Bildungstechnologien, eine gewisse Entscheidungsfreiheit beim Medieneinsatz und Austauschmöglichkeiten mit Kollegen und Kolleginnen zum Medienumgang in Zusammenhang mit der Motivation und der Entwicklung einer positiven Einstellung gegenüber der Nutzung digitaler Medien stehen (Kreijns et al., 2014, S. 475). Somit weisen die Resultate darauf hin, dass die Einstellung als wesentlicher Faktor der persönlichen Haltung eine sehr wichtige Variable für die Nutzung digitaler Medien darstellt; und dass die Befriedigung der Grundbedürfnisse beim Umgang mit digitalen Lernmaterialien im schulischen Alltag erforderlich ist, um die Lehrkräfte zu motivieren und eine positive Einstellung gegenüber der Verwendung digitaler Lernmaterialien zu entwickeln (Krejns et al., 2014, S. 476).

\subsection{Zur Kompetenz des pädagogischen Personals}

Der Begriff der Kompetenz und die Kompetenzentwicklung von Lehrpersonen und Ausbildungspersonal im Umgang mit digitalen Medien und Techniken erhalten im Hinblick auf die Verbesserung der Lehr- und Lernqualität im Kontext des Einsatzes digitaler Medien eine besondere Bedeutung. Allerdings ist die Definition der Begriffe Medienkompetenzen oder digitale Kompetenzen nicht ganz einfach, da „inhaltliche Beschreibungen wie Medien, Computer, IKT [Informationsund Kommunikationstechnik; Anm. der Autorinnen] mit Bildungskonzepten wie Fertigkeiten, Literarität (Literacy) und Kompetenz miteinander verknüpft werden 
(müssen)“ (Baumgartner, Brandhofer, Ebner, Gradinger \& Korte, 2016, S. 95). Die EU stellt seit einiger Zeit den Begriff „digitale Kompetenz“ in den Vordergrund, weil er weiter gefasst erscheint und den engen Bezug zu dem Begriff „Medien“ ein Stück weit aufhebt (vgl. Baumgartner et al., 2015, S. 96 f.). Damit orientiert sie sich an der Definition von Ferrari (2012), welche die digitale Kompetenz als ein Set aus Wissen, Fertigkeiten und Einstellungen beschreibt, das notwendig ist, um IKT und digitale Medien zur Aufgabenerfüllung, zur Problemlösung, zur Kommunikation, zur Verwaltung von Informationen, zur Zusammenarbeit, zur Erstellung und zum Austausch von Inhalten sowie zum Aufbau von Wissen einzusetzen (Ferrari, 2012, S. 3). In der deutschen Diskussion werden weiterhin Begriffe wie „(digitale) Medienkompetenzen“ oder „Kompetenzen in der digitalen Welt“ oder „medienpädagogische Kompetenz" mit Bezug auf das pädagogische Personal genutzt (Breiter, Howe \& Härtel, 2017, S. 34 ff; Härtel et al., 2018a; KMK, 2017). Im Folgenden werden die Kompetenzbegriffe der jeweiligen Studien oder der Begriff digitale Kompetenzen genutzt.

Sechs Studien befassen sich näher mit digitalen Kompetenzen des pädagogischen Personals in der Berufsbildung. Zwei Publikationen lassen sich der Modell- und Instrumentenentwicklung zur Erfassung digitaler Kompetenzen des pädagogischen Personals zuordnen. Härtel et al. (2018b) haben ein Modell medienpädagogischer Kompetenz für das betriebliche Ausbildungspersonal entwickelt, dessen drei Kompetenzkomponenten Mediendidaktik, Medienerziehung und Medienintegration in einer Wechselbeziehung zueinanderstehen. Das Modell ist Ergebnis eines mehrstufigen Entwicklungsprozesses. In einem ersten Schritt erfolgte die Entwicklung eines Prototyps mit Bezugnahme auf einen medienpädagogischen Theorierahmen und auf ein etabliertes Modell aus der Allgemeinbildung (Blömeke, 2000; Tulodziecki, 2010), welches insbesondere in der Lehramtsausbildung verortet werden kann. Mittels zehn Experteninterviews mit betrieblichem Ausbildungspersonal ausgewählter Domänen (gewerblich-technisch: Industriemechatroniker/Industriemechatronikerin, Kfz-Mechatroniker/Kfz-Mechatronikerin; Handel: Einzelhandelskaufmann/Einzelhandelskauffrau; Pflege: Altenpfleger/Altenpflegerin) sowie Forschenden der Berufsbildung wurde das Modell ergänzt und überprüft. Die Optimierung des Prototyps und Modelldifferenzierung erfolge auf Grundlage der Interviewergebnisse. Das Modell bildet die Grundlage für eine anschließend durchgeführte Online-Befragung (Laufzeit: 05-09/2016) von betrieblichem Ausbildungspersonal (N=733). Zur Vertiefung und Reflexion dieser Erkenntnisse im Kontext der jeweiligen beruflich-betrieblichen und institutionellen Umfelder erfolgten dann Gruppeninterviews sowie Workshops mit Expertinnen und Experten in vier domänenspezifischen Gruppen mit insgesamt 17 Teilnehmenden.

Zielgruppe des Rahmenkonzeptes zur Erfassung digitaler Kompetenzen von Seufert et al. (2019) sind hingegen Lehrpersonen. Das Konzept umfasst die Kompetenzfacetten Fachwissen, fachdidaktisches sowie pädagogisches Wissen, Beratungs- und Organisationswissen, instrumentelle Fertigkeiten im Umgang mit 
digitalen Medien und Einstellungen. Es orientiert sich am Konzept der professionellen Kompetenz von Baumert und Kunter (2006) und bezieht Modellelemente von Koehler und Mishra (2009), Blömeke (2003) und Carretero et al. (2017) mit ein. Ebenso wie bei Valtonen et al. (2013) nutzen die Forschenden mit dem TPACK-Modell auch ein spezifisches Kompetenzkonzept im Umgang mit digitalen Medien. Das Rahmenkonzept wurde wie bei Härtel et al. (2018b) mehrstufig entwickelt. Der erste Schritt bestand in einer systematischen Literaturanalyse von 83 verfügbaren Studien zur Entwicklung digitaler Kompetenzen. Hierbei wurden sowohl berufsfeldübergreifende als auch spezifisch auf kaufmännische Berufsfelder bezogene Untersuchungen berücksichtigt. Für ein theoretisches Sampling in der Schweizerischen Berufsbildung fand als nächstes eine Befragung von Ausbilderinnen und Ausbildern in zehn ausgewählten Betrieben aus fünf Branchen statt, in denen kaufmännische Berufe stark vertreten sind. Anschließend wurden zwölf Expertinnen und Experten zur schweizerischen Berufsbildung sowie zur Technikfolgenabschätzung befragt. Ende 2017 erfolgte die Datenerhebung mit der das Rahmenkonzept validiert wurde. An dieser Online-Befragung nahmen 215 Lehrkräfte aus neun kaufmännischen Schulen der Deutschschweiz teil.

Seufert et al. (2019) konstatieren, dass das „mediendidaktische Wissen“ der befragten Lehrpersonen steigerungsbedürftig ist und stellen - wie bereits zur Haltung des pädagogischen Personals - eine Abhängigkeit vom Alter bei dieser Kompetenzausprägung fest. Diese ist bei den 56- bis 65-Jährigen im Vergleich zu unter 36-Jährigen niedriger. Keine signifikanten Unterschiede gibt es jedoch im Vergleich der Altersgruppen 36-55 und unter 36. Die fehlende empirische Trennbarkeit zwischen den Kompetenzausprägungen "Fachwissen“ und „fachdidaktischem Wissen“ interpretieren sie als möglichen Hinweis darauf, dass die Befragten möglicherweise eine geringe fachdidaktische Expertise im Bereich der digitalen Medien haben.

Wie bereits im Abschnitt zur didaktisch-methodischen Perspektive deutlich wurde, ist der Einsatz von technischen Geräten und digitalen Lernmedien in der betrieblichen und schulischen Ausbildungspraxis sehr unterschiedlich. Dem aktuellen Forschungsstand zufolge steigern vorhandene Kompetenzen im Umgang mit digitalen Bildungstechnologien die Motivation beim Einsatz digitaler Lernmaterialen (Kreijns et al., 2014, S. 475). Gleichzeitig werden Kompetenzen der Bedienbarkeit auch erst durch konkrete Nutzung auf- und ausgebaut. Härtel et al. (2018b) haben in ihrer Befragung Daten zur Nutzungs- und Bedienkompetenz erhoben. Bei der Nutzung gängiger Geräte (z.B. PC, Beamer) und Anwendungen (z.B. OfficeProgramme, E-Mail, Suchmaschinen/Internetrecherchen) fühlt sich ein Großteil des betrieblichen Ausbildungspersonals sehr sicher oder sicher (80-90\%). Ebenso gaben über $90 \%$ der Befragten an, dass sie Beamer, PC und Laptop sicher oder sehr sicher bedienen können (Härtel et al., 2018b, S. 30 f.). Sicherheit bei der Bedienung besteht also genau bei den Geräten, die besonders häufig in der betrieblichen und schulischen Ausbildungspraxis verwendet werden. 
Neuere Medientechnik und Hardware setzt das Lehr- und Ausbildungspersonal hingegen eher selten ein (Schmid et al., 2016, S. 31). Interaktive Whiteboards und Smartwatches haben $46 \%$ bzw. $66 \%$ der Ausbilderinnen und Ausbilder in der Befragung von Härtel et al. (2018b) noch nie verwendet. Folglich ist auch der Anteil derer, die dieses sicher nutzen können, gering: Nur $20 \%$ bzw. $13 \%$ fühlen sich sehr sicher oder sicher in der Nutzung dieser Techniken. Hierbei ist aber auch zu bedenken, dass Geräte wie Whiteboards nicht in allen Betrieben vorhanden sind. Beim Umgang mit Cloudanwendungen fühlt sich nur ein Anteil von $35 \%$ sicher oder sehr sicher. Etwa ein Viertel (26\%) der Befragten hat eine Cloud noch nie genutzt.

Die Nutzung von Social Media in der Ausbildungspraxis steigt zwar an, ist aber durchaus sehr unterschiedlich verbreitet. In der Nutzung sozialer Netzwerke fühlt sich ein Anteil von $42 \%$ des Ausbildungspersonals sehr sicher oder sicher. Etwas mehr als ein Viertel der Befragten (27\%) hat in im Rahmen der Ausbildungstätigkeit noch nicht mit sozialen Netzwerken gearbeitet. (Härtel et al., 2018b)

Eine kritische-reflektierte Behandlung gesellschaftlicher und individueller Bedeutung digitaler Medien in der Ausbildung wurde von Härtel et al. (2018b) als wichtiger Kompetenzbereich festgelegt und mit einem Bündel Fragestellungen erhoben. Hier zeigt sich, dass zwar $83 \%$ des befragten Ausbildungspersonals selbst die Chancen und Risiken im Umgang mit digitalen Medien kennen, aber nur etwa die Hälfte hiervon (42\%) hat zudem ein Bewusstsein dafür, inwieweit sich auch die Auszubildenden damit auskennen. Insgesamt $58 \%$ thematisieren die Chancen und Risiken in der Ausbildung.

Für die Förderung der Digitalisierung werden von Sloane et al. (2018) den organisatorischen Kompetenzen und selbstregulativen Fähigkeiten des pädagogischen Personals eine hohe Bedeutung zugeschrieben. Härtel et al. (2018b) haben die befragten Ausbilderinnen und Ausbilder um eine Selbsteinschätzung zu betrieblichen und rechtlichen Rahmenbedingungen des Einsatzes digitaler Medien gebeten. Ein Anteil von $82 \%$ der Teilnehmenden kennt betriebsinterne Regelungen zur Nutzung digitaler Medien und $68 \%$ wissen, welche Regelungen zum Copyright bei der Entwicklung von Lernmaterialien beachtet werden müssen. Auch Datenschutzregeln sind sehr gut bekannt. Betriebliche Rahmenbedingungen zum Einsatz digitaler Lernmedien sind immerhin noch $63 \%$ bekannt. Allerdings gibt es in den Ausbildungsbetrieben bei $49 \%$ der Befragten keine betrieblichen Richtlinien zum Einsatz digitaler Medien in der Ausbildung.

\subsection{Weiterbildungsbedarf, -angebot und -nachfrage}

Trotz positiver Einschätzungen der fachlichen Kompetenzen von Lehrpersonen und betrieblichem Ausbildungspersonal wird in verschieden Studien ein deutliches Steigerungspotenzial bei der Entwicklung digitaler Kompetenzen diagnostiziert (BIBB, 2014; Härtel et al., 2018b; Kreijns et al., 2014; Schmid et al., 2017; Seufert et 
al., 2019; Sloane et al., 2018). Ungefähr die Hälfte der befragten Ausbilderinnen und Ausbilder der Nutzerbefragung des Portals foraus.de (BIBB, 2014) gibt selbst an, dass für den Einsatz digitaler Elemente in der Ausbildungspraxis weitere Fortbildungen benötigt werden. Nur ein Viertel des betrieblichen Ausbildungspersonals in der Befragung von Härtel et al. (2018b) kennt überhaupt Konzepte der Medienerziehung. Seufert et al. (2019) stellen fest, dass das mediendidaktische Wissen bei den 56 bis 65-jährigen Lehrpersonen im Vergleich zu den unter 36-jährigen Lehrpersonen niedriger ausgeprägt ist. Härtel et al. (2018b, S. 28) stellen hingegen keine signifikanten Kompetenzunterschiede nach Altersgruppen fest.

Lehrende, die an schulisch initiierten Fortbildungen zum Einsatz digitaler Lernprogramme teilnehmen, weisen signifikant höhere Ausprägungen bei den Kompetenzfacetten allgemeinen pädagogischen Wissens, Wissen über die Förderung digitaler Fertigkeiten, mediendidaktisches Wissen, Beratungs- und Organisationswissen auf (Seufert et al., 2019). Jedoch nehmen nur knapp $50 \%$ der Lehrkräfte und Ausbilderinnen und Ausbilder überhaupt an formalen Weiterbildungskursen teil (Schmid et al., 2016; ähnlich: Härtel et al., 2018b). Und diese unterscheiden sich nach Einschätzungen von Expertinnen und Experten erheblich in der Qualität.

Die bislang wichtigste wahrgenommene Weiterbildungsgelegenheit ist mit $80-$ 90 \% (Schmid et al., 2017), bzw. 73 \% (Härtel et al., 2018b) der informelle Austausch des pädagogischen Personals untereinander zum Einsatz digitaler Medien sowie zu Funktionen von Geräten und digitalen Anwendungen. In den betrieblichen Fallstudien von Jablonka et al. (2018, S. 116) wird berichtet, dass Unternehmen u. a. vor dem Hintergrund der zunehmenden Digitalisierung gegenwärtig regelmäßige bundesweite Netzwerktreffen des Ausbildungspersonals einrichten. Ebenfalls kommt der autodidaktischen Aneignung eine hohe Bedeutung zu. Nach Schmid et al. (2016) bilden sich durch Selbststudium $94 \%$ der befragten Lehrkräfte und immerhin $62 \%$ der befragten Ausbilderinnen und Ausbilder weiter. Auch in der Untersuchung von Härtel et al. (2018b) geben $44 \%$ der Befragten an, Fachzeitschriften zur Wissensaneignung $\mathrm{zu}$ nutzen. Internetplattformen, wie beispielsweise das Portal foraus.de für betriebliches Ausbildungspersonal, nutzen mit 55\% etwas mehr als die Hälfte der Teilnehmenden. Eine Befragung des BIBB (2014) ( $N=376)$ zu seinem Angebot foraus.de ergab, dass es 1/3 der Befragten kennen und es $17 \%$ einmal pro Woche nutzen. Fast alle (94\%) nutzen das Portal zur Informationssuche, $60 \%$ zum Download von Dokumenten und Materialien, $25 \%$ zum Lernen und $15 \%$ zum Austausch mit anderen Ausbilderinnen und Ausbildern. Zwischen der Nutzung des Forenbereiches und dem Alter besteht ein schwacher Zusammenhang. Tendenziell wird das Forum eher von Jüngeren genutzt.

Es gibt bereits Projekte, die Forschung zur Weiterbildung des pädagogischen Personals im Digitalisierungskontext betreiben und hiermit die Entwicklung entsprechender Maßnahmen und Angebote verknüpfen. Beispielweise wurde im Rahmen eines Forschungsprojekts, indem es um den Medieneinsatz in der Schweißausbildung ging, ein Train-the-Trainer Konzept entwickelt. Betriebliches Ausbildungspersonal 
arbeitete in Präsenztrainings mit Trainerinnen und Trainern zusammen an der Umsetzung von Unterstützungs- und Bildungsangeboten innerhalb von Arbeitsprozessen in den jeweiligen Organisationen, z.B. als Lernprojekte im Sinne eines lernortübergreifenden Ansatzes. Dabei wurden Lehr-Lern-Arrangements projektförmig aufgebaut und das prozess- und aufgabenorientierte Lehren und Lernen in problemhaltigen Situationen und in einem beruflichen Kontext erprobt. Zusätzlich wurden Video-Tutorials zur Verwendung von Schweißsimulatoren in unterschiedlichen Settings entwickelt und auf einer Lernplattform bereitgestellt, um die Medienkompetenz des Ausbildungspersonals zu optimieren (BIBB, 2018/2019, S. 25 f.).

Autorinnen und Autoren mehrerer Studien leiten aus ihren Forschungsergebnissen Handlungsempfehlungen zur Weiterbildung des pädagogischen Personals ab. Schmid et al. (2016) kommen zu dem Schluss, dass Weiterbildungsangebote stärker auf das Engagement und die intrinsische Motivation der Lehrkräfte abzielen sollten. Seufert et al. (2019) plädieren für eine Entwicklung von Fördermaßnahmen, z. B. im Blended-Learning-Format, die ebenso auf die eigene Motivation der Lernenden setzen. Härtel et al. (2018b, S. 66 ff.) haben konkrete inhaltliche Schussfolgerungen aus der Bedarfserhebung im Rahmen ihrer Forschung entwickelt. Als sinnvoll und notwendig erachtet werden beispielsweise die „Entwicklung domänenspezifischer Best-Practice-Angebote zur Sicherung der fachlich-inhaltlichen Qualität der Ausbildungsmedien“ (Härtel et al., 2018b, S. 66; ähnlich: Schmid et al., 2016), „Informationen und Weiterbildungsangebote zum Medienrecht, Datenschutz und zur Datensicherheit zur Sicherung von rechtskonformen Handeln in der Ausbildungspraxis“ (Härtel et al., 2018b, S. 67) sowie „Weiterbildungsangebote zum Aufbau medienpädagogischer Kompetenz als Beitrag zum systematischen Aufbau dieser Kompetenz in der Ausbildung des Ausbildungspersonals“ (Härtel et al., 2018b, S. 67). Eine ebenfalls detaillierte, aber weniger inhaltlich konkretisierte Empfehlung geben Sloane et al. (2018, S. 142) mit ihrem Vorschlag zum Aufbau regionaler Strukturen zur Förderung von Digitalisierung und digitalen Kompetenzen, die wiederum der Förderung regionaler Netzwerke in der Berufsbildung dienen sollen. Sie schlagen ein "Agenturmodell“ vor, dass verschiedene Aufgabenfelder übernimmt (Anlaufstelle für Bildungseinrichtungen, Erstellen von Qualifizierungsmaßnahmen, Förderung regionaler Kooperationen, Lieferung von Umsetzungshilfen für betriebliche Bildungspläne und schulische Jahrespläne). Angesetzt werden solle an der pädagogischen Professionalität des Bildungspersonals. Im Sinne einer regionalen Strukturförderung werden nicht nur Einzelne individuell unterstützt, sondern ein gemeinsames didaktisches Verständnis zur Nutzung digitaler Lernmittel erarbeitet. 


\subsection{Institutionelle Verankerung von digitalen Lernanwendungen und Medientechnik}

Die Anwendung digitaler und digitalisierter Komponenten in der Bildungsorganisation und -praxis der beruflichen Ausbildung hängt von unterschiedlichen Faktoren und Rahmenbedingungen ab. Im Studien-Sample gehen fünf Publikationen näher auf die institutionelle Verankerung digitaler Lernanwendungen und Medientechnik ein. Auffällig erscheint, dass kaum Gelingensbedingungen formuliert oder herausgearbeitet werden, sondern vordergründig die Hemmnisse beim Einsatz digitaler Medien aufgeführt sind. Hierbei nehmen fehlende Kompetenzen des pädagogischen Personals eine besonders zentrale Rolle ein (vgl. Abschnitt 3.3). Erschwerend kommt hinzu, dass medienpädagogische Kompetenzen aufgrund fehlender zeitlicher Ressourcen nicht aufgebaut werden können. Eine tiefergehende Beschäftigung mit digitalen Lernmedien ist nicht möglich. Schulleitungen, Berufsschullehrkräfte und Ausbildungsverantwortliche konstatieren einen - zumindest beim Einstieg - deutlich erhöhten zeitlichen Aufwand, der mit der Verwendung von digitalen Lernformaten und der Nutzung entsprechender Geräte einhergeht (Schmid et al., 2016). Zur Kompensation der zeitlichen Mehraufwände fehlen bisher entsprechende Regelungen (Schmid et al., 2016). Die Unternehmensgröße bestimmt hierbei Möglichkeiten der Einführung neuer Anwendungen und Geräte wesentlich mit. Große Unternehmen haben mehr zeitliche und personelle Ressourcen, um den Medieneinsatz anzupassen oder zu verändern. Allerdings erfordert es dort auch die Einbeziehung mehrerer Entscheidungsebenen und ist häufig mit einer standortübergreifenden Einführung („Rollout") verbunden. Es scheint, dass kleine und mittlere Unternehmen (KMU) hingegen flexibler und spontaner auf äußere Einflüsse reagieren können, da die Abstimmungswege kürzer sind; allerdings sind sie mangels zeitlicher und personeller Ausstattung in ihrer Ausbildungsplanung weniger gut aufgestellt. (Härtel et al., 2018b, S. 57) Auch auf didaktischer Ebene, insbesondere der fachdidaktischen Ebene, bestehen durch ein mangelndes Angebot und/ oder einer mangelnden Qualität von Lernprogrammen sowie der Unübersichtlichkeit der Angebote hinderliche Bedingungen.

Häufig liegen die eingangs beschriebenen Einschränkungen in kombinierter Form vor. Beispielweise führen Internetrecherchen der Lernenden zu unterschiedlichen Ergebnisqualitäten, da nicht alle über entsprechende Kompetenzen verfügen, um seriöse Webseiten aus dem unübersichtlichen Angebot herauszufiltern. Das pädagogische Personal sieht es daher als wichtige Aufgabe an, eine Qualitätssicherung durchzuführen, die wiederum sehr viel Zeit in Anspruch nimmt. (u. a. Schmid et al., 2016; Sloane et al., 2018) Für einem Teil des Ausbildungspersonals ist das klassische Lehrbuch daher das verlässlichere Medium (z. B. Jablonka et al., 2018).

Ein sehr deutliches und studienübergreifendes Hemmnis der institutionellen Verankerung digitaler Lernanwendungen und Medientechnik stellen die Rahmenbedingungen und Infrastrukturen von Berufsschulen, Ausbildungsbetrieben 
und überbetrieblichen Ausbildungsstätten dar: Technische Probleme bzw. die mangelnde oder nicht zeitgemäße Ausstattung der Lernorte stehen dem Einsatz digitaler Medien entgegen. Hier spielen $u$. U. auch der Datenschutz und das bestehende Arbeitsrecht eine Rolle (Härtel et al., 2018b; Schmid et al., 2016; Sloane et al., 2018). Sloane et al. (2018) sprechen von einer Komplementarität von pädagogisch-didaktischer sowie organisatorischer Kompetenz, die es auszubauen gilt. So hat nur etwa die Hälfte der Betriebe Richtlinien zum Einsatz digitaler Medien in der Ausbildung entwickelt (Härtel et al., 2018b). Auch auf schulischer Seite gibt es Defizite. Im DGB-Ausbildungsreport (2019) bewerten nur 34,9\% der befragten Auszubildenden die digitale Ausstattung ihrer Berufsschule mit sehr gut oder gut. Die Studie verweist auf einen Zusammenhang zwischen Bewertungen zur digitalen Ausstattung der Berufsschulen, zur Vorbereitung an den Berufsschulen auf den Umgang mit digitalen Medien und Technologien und der fachlichen Qualität des Unterrichts. Die Qualität des WLANs schätzen nur 38\% der befragten Lehrkräfte als sehr gut bis gut ein, und $40 \%$ geben an, dass an ihrer Schule kein WLAN vorhanden ist (Schmid et al., 2016). In einer landesweiten Umfrage zur IT-Ausstattung und Medienbildung der Schulen in Schleswig-Holstein ${ }^{9}$ stellt sich die Infrastruktur an berufsbildenden Schulen (in SH) allerdings deutlich besser dar als an allgemeinbildenden Schulen (IQSH, 2018, S. 13). In diesem Bundesland verfügen alle befragten berufsbildenden Schulen über ein fest installiertes WLAN, bei $73,3 \%$ ist das WLAN-Netz auf dem gesamten Schulgelände (schulweit) erreichbar. Gegenüber anderen Schulformen ist die Computer-Schüler-Relation besser, es haben mehr berufliche Schulen ein eigenes Budget für IT sowie Regelungen zur Erneuerung der ITAusstattung als der Durchschnitt aller Schulen in Schleswig-Holstein (vgl. Tabelle 3). Auch in der Nutzung von Online-Systemen zum Materialaustausch, für Hausaufgaben und Unterrichtsergebnisse, für Klassenarbeiten oder auch für das elektronische Klassenbuch sind Lehrkräfte an beruflichen Schulen deutlich aktiver als an anderen Schulen (IQSH, 2018, S. 27). Den großen Unterschied bei der Festlegung für Erneuerungen der IT-Ausstattung zwischen berufsbildenden Schulen und allen Schulen erklären die Autorinnen und Autoren der Umfrageergebnisse mit den besonderen Anforderungen der beruflichen Schulen auf Grund ihres Bildungsauftrages in der beruflichen Ausbildung (IQSH, 2018, S. 23).

9 An der Online-Befragung von Schulleitungen zwischen März und Juni 2018 haben sich 30 von 33 beruflicher Schulen oder regionaler Berufsbildungszentren (RBZ) beteiligt und rund 40 Fragen zur Ausstattung und Nutzung von Informationstechnologien sowie Fragen zur Konzeption und Entwicklung von Medienbildung an ihren Schulen beantwortet. 
Tabelle 3: Ausstattung und Nutzung von Informationstechnologien; Vergleich berufsbildende Schulen/Berufsbildungszentren und Durchschnitt aller Schulformen in SchleswigHolstein

Datenquelle: IQSH 2018; eigene Darstellung

\begin{tabular}{lcc}
\hline & BBS / RBZ & $\begin{array}{c}\text { alle Schul- } \\
\text { formen }\end{array}$ \\
\hline fest installiertes WLAN & $100 \%$ & $76,7 \%$ \\
WLAN-Empfang im gesamten Schulgebäude & $73,3 \%$ & $30,7 \%$ \\
Computer-Schüler-Relation & 1 zu 6,2 & 1 zu 8,7 \\
Schule hat IT-Budget & $36,7 \%$ & $23,7 \%$ \\
Festlegung für regelmäßige Erneuerung der IT-Ausstattung vorhanden & $70 \%$ & $24,2 \%$ \\
\hline
\end{tabular}

Auch bei der institutionellen Verankerung von digitalen Lernanwendungen und Geräten bestehen (wieder) Unterschiede nach Ausbildungsberufen. Apps, z.B. zum Lernen oder zur Dokumentation, nutzen insgesamt nur etwas mehr als ein Fünftel der Auszubildenden im DGB-Ausbildungsbericht. Überdurchschnittlich verbreitet sind solche Apps bei den Bankkaufleuten mit 59,2\% und den Fachinformatikerinnen und -informatikern mit 48,2\% (DGB, 2019, S. 15). Technische Geräte erhält nur etwa jeder Vierte $(26,4 \%)$ der Befragten vom Ausbildungsbetrieb zur Verfügung gestellt. Auch hier tun sich die angehenden Fachinformatikerinnen und Fachinformatiker mit 69,2\% wieder deutlich hervor. Auszubildende der Berufe Mechatroniker/Mechatronikerin, Elektronikerin für Betriebstechnik/Elektronikerin für Betriebstechnik, Industriekaufleute und Kaufleute für Büromanagement werden ebenfalls relativ häufig (zwischen 39 und $47 \%$ ) mit technischen Geräten ausgestattet (DGB, 2019, S. 16).

Insgesamt werden in der schulischen Bildungsorganisation und -praxis jedoch anscheinend keine strategisch-ganzheitlichen Konzepte zum Einsatz von Lernmedien genutzt (Schmid et al., 2016). Eine Betrachtung der initiierenden Akteure im Monitor Digitale Bildung (Schmid et al., 2016) zeigt auf, dass nur ca. $30 \%$ der Maßnahmen auf die Initiative von Schulleitungen zurückgehen. Schulträger oder Landesregierungen treten sogar nur bei $5 \%$ der Aktivitäten als Initiatoren auf. Die Organisation und Sicherstellung der technischen Umsetzungsmöglichkeiten stützen sich zu einem Großteil auf die Lehrkräfte (80\%). Nur bei $50 \%$ der Berufsschulen gibt es eine externe Mitarbeiterin oder einen externen Mitarbeiter für die Betreuung von Medien und IT (Schmid et al., 2016). Weiterhin ist eine moderne Ausstattung alleine nicht ausreichendend, denn auch der technische Support, die Wartung und eine zyklische Modernisierung der Ausstattung müssen ebenso sichergestellt sein. Hierbei handelt es sich um Aufgaben, die nicht nebenbei von Lehrkräften übernommen werden können (z. B. Sloane et al., 2018). 


\section{Fazit: Aktuelle Entwicklung sowie Handlungs- und Forschungsbedarfe}

Der zunehmende Stellenwert digitaler Medien für die (Weiter-)Entwicklung von Bildungsangeboten in der Berufsbildung zeigt sich in den beschriebenen Studien und deren Ergebnissen sowie den aktuellen Diskussionen in der Bildungspolitik (Vollmer, Jaschke \& Schwenger, 2017). Ihr zunehmender Einsatz in der Berufsbildungspraxis stellt das pädagogische Personal vor die große Herausforderung, sich umfangreiche digitale Medienkompetenzen anzueignen. Allerdings werden digitale Bildungsformate derzeit noch recht uneinheitlich mehr oder weniger stark für die Unterrichtsgestaltung genutzt, obwohl sie im Zuge des technologischen Fortschritts in den nächsten Jahren - bildungspolitisch gewollt - weiter ausgebaut werden sollen. Die Kultusministerkonferenz hat bereits im Jahr 2017 auf Basis des Beschlusses zur „Bildung in der digitalen Welt“ (Dezember 2016) ein bildungssektorenübergreifendes Strategiepapier herausgebracht. Dieses beschreibt einerseits den Bildungsauftrag von Schulen der Berufsbildung in der digitalen Welt und thematisiert andererseits auch die Umsetzung von Bildungsaufgaben sowie die notwendigen begleitenden Maßnahmen beispielsweise die Aus- und Weiterbildung von Lehrkräften oder die Verfügbarkeit von digitalen Medien (KMK, 2017, S. 20 ff.). In Abgrenzung zu den allgemeinbildenden Schulen wird dabei in den Fokus gerückt, dass berufsbildende Schulen eine Art „Wandlungskompetenz“ entwickeln müssen, mit der sie - aufgrund ihrer besonderen Nähe zur Arbeitswelt - zeitnah auf die Anforderungen durch Digitalisierung reagieren und aktuelle Entwicklungen in die Unterrichtsgestaltung einbinden können. Eine berufsspezifische Vermittlung und Weiterentwicklung von fachübergreifenden digitalen Kompetenzen wie der Einsatz und die Anwendung von Medien- und Arbeitstechniken oder der kritische Umgang mit Medien, sind in diesem Kontext als Querschnittaufgaben zu sehen (KMK, 2017, S. $20 \mathrm{f}$.). Für die Lehrkräfte heißt das, dass sie zur Bewältigung dieser Aufgabe, allgemeine umfassende digitale Medienkompetenzen aufbauen müssen, um als „Medienexperten“ (KMK, 2017, S. 20f.) eine an den technologischen Fortschritten orientierte berufsspezifisch geprägte Medienbildung im Unterricht gewährleisten zu können. Diese Medienbildung sollte sich - z. B. im Falle dualer Berufsausbildungen - an den jeweils aktuellen Fassungen der bundeseinheitlich geregelten Ausbildungsordnungen zu den Ausbildungsberufen orientieren.

Ein Blick auf die Förderlandschaft verdeutlicht, dass sich die bundesweite Förderung von Projekten zu Themen der digitalen Bildung und $\mathrm{zu}$ ihrem Einsatz in Schulen sowie in Unternehmen insbesondere seit 2011 weiter ausgedehnt hat. Die BMBF-Förderlinie „Stärkung der digitalen Medienkompetenz für zukunftsorientierte Medienbildung in der beruflichen Qualifizierung "10 (zwei Förderphasen, Laufzeit seit 2011) unterstützt Forschungsprojekte, die die Verankerung von Medienbildung

10 https://www.bmbf.de/foerderungen/bekanntmachung.php?B=679 und https://www.bmbf.de/foerderungen/bekanntmachung-1137.html (zuletzt aufgerufen am 27.01.2020) 
und die Kompetenzförderung in der beruflichen Aus- und Weiterbildung sowie in Bildungsangeboten des Übergangssystems im Blick haben. Zur Optimierung der Anwendung digitaler Medien in der beruflichen Bildung hat das BMBF das mit Mitteln des Europäischen Sozialfonds kofinanzierte Förderprogramm „Digitale Medien in der beruflichen Bildung" ${ }^{111}$ (Laufzeit 2012-2019) initiiert. Dabei werden Projekte unterstützt, die übergreifende, digitale Bildungsangebote schaffen, modularisierte Lernangebote beinhalten, den Transfer zum Nutzen in die Bildungspraxis fördern oder den Einsatz mobilen Lernens im Fokus haben. Seit 2016 setzen BMBF und BIBB gemeinsam die „Initiative Berufsbildung 4.0"12 um, die die zahlreichen Programme unter einem Dach vereint und Forschungsprojekte berücksichtigt, die die Auswirkungen der Digitalisierung auf Qualifikations- und Kompetenzbedarfe und den Einsatz digitaler Medien in der Berufsbildung untersuchen. Ganz aktuell sind der Digitalpakt Schule ${ }^{13}$ (Laufzeit 2019-2024) und die aktuelle BMBF-

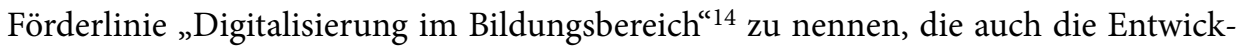
lungen in der beruflichen Bildung berücksichtigen.

Die aufgeführten aktuellen Entwicklungen sowie die ausdifferenzierte Förderund Forschungslandschaft spiegeln sich jedoch weder in der Quantität der Studienlage noch in der Qualität der beruflichen Ausbildungspraxis im Umgang mit Bildungstechnologien wider. Nur wenige in diesem Dossier beschriebene Studien basieren auf Ergebnissen zu den Projekten der benannten Förderlinien. Zudem sind die Kompetenzen des Lehr- und Ausbildungspersonals beim Umgang mit digitalen Medien insgesamt bisher noch sehr unterschiedlich stark ausgeprägt. Zahlreichen Ausbildungs- und Lehrkräften mangelt es an tiefergehenden Kompetenzen in Bezug auf die Anwendung digitaler Medien. Daher entspricht ihr Einsatz im Unterricht und Ausbildungsgeschehen nicht den hohen Erwartungen und den Anforderungen einer digitalisierten Arbeitswelt. Auch bei der infrastrukturellen Ausstattung können die beruflichen Bildungsinstitutionen kaum Schritt halten.

Hieraus ergeben sich konkrete Forschungs- und Handlungsbedarfe:

- Im Vergleich zu anderen Bildungsbereichen erscheint der Forschungsstand in der Berufsbildung eher einseitig auf die duale Berufsausbildung bezogen. Andere Bereiche wie die Ausbildungsvorbereitung oder die Bildungsgänge der schulischen Berufsbildung werden wenig beleuchtet. Die Auswertungen verbleiben meist auf deskriptiver Ebene. Außerdem weisen die bestehenden Studien häufig sehr geringe Fallzahlen auf, was die Repräsentativität der Ergebnisse einschränkt.

- Es ist zu konstatieren, dass es bisher keine einheitlich geregelten strategischen Konzepte im Umgang mit digitalen Medien in der Berufsbildung gibt. Die Länder

11 https://www.bmbf.de/de/digitale-medien-in-der-bildung-1380.html (zuletzt aufgerufen am 27.01.2020)

12 https://www.bmbf.de/upload_filestore/pub/Berufsbildung_4.0.pdf (zuletzt aufgerufen am 27.01.2020)

13 https://www.bildung-forschung.digital/de/der-digitalpakt-schule-kommt-2330.html (zuletzt aufgerufen am 27.01.2020)

14 https://www.qualifizierungdigital.de (zuletzt aufgerufen am 08.05.2020) 
verfolgen unterschiedliche Digitalisierungsstrategien in der Bildung, was zu einem föderalen Flickenteppich bei der Ausgestaltung von Medienkonzepten in berufsbildenden Schulen führt. Auch die infrastrukturellen Rahmenbedingungen für den Einsatz digitaler Medien unterscheiden sich je nach Schule. Somit sind die Ressourcen, die dem pädagogischen Personal für den Umgang mit digitalen Medien zur Verfügung stehen sowohl innerhalb der Länder als auch länderübergreifend ungleich verteilt.

- Studienübergreifend lässt sich festhalten, dass eine bedarfsgerechte Qualifizierung der Lehrkräfte sowie des Ausbildungspersonals fehlt. Es gibt weder flächendeckende Qualifizierungsangebote, noch scheinen Fort- und Weiterbildungsformate zum Einsatz digitaler Medien in den Bildungsinstitutionen und Ausbildungsbetrieben in ausreichendem Maße vorhanden und strukturell verankert zu sein. Hier bestehen aus Sicht von Experten und Expertinnen große Defizite (Härtel et al., 2018b; Schmid et al., 2016, S. 21 f.).

- Weitere wesentliche Gründe liegen in der mangelnden technischen Ausstattung für einen adäquaten Einsatz digitaler Medien im Unterrichts- und Ausbildungsgeschehen, weil dieser häufig mit zu hohen Kosten verbunden ist. Die Ergebnisse aus den zentralen Studien zeigen, dass die Ausstattung mit der Medientechnik/Hardware nicht zeitgemäß ist, ein flächendeckendes WLAN-Netz in den berufsbildenden Schulen häufig fehlt und in den Schulen meistens keine IT-Fachleute existieren, die bei technischen Problemen oder der Wartung der Geräte unterstützend helfen können. (BIBB, 2018, S. 83 ff.; IQSH, 2018; Schmid et al., 2016, S. 21 f.; Sloane et al., 2018).

- Es existierten derzeit zu wenige Studien, die die Thematik Aus- und Fortbildung für das Lehr- und Ausbildungspersonal aufgreifen. Das stellt den Bereich der beruflichen Bildung vor die Problematik, dass es derzeit keine adäquaten Konzepte gibt, die für eine angemessene Weiterqualifizierung des pädagogischen Personals genutzt werden. Außerdem müssten die Rahmenbedingungen dahingehend verändert werden, dass zeitliche Ressourcen für die Aus- und Fortbildung geschaffen werden. Auch die Option, das Thema Digitalisierung in der Ausbildereignungsverordnung (AEVO) unterzubringen, könnte überdacht werden. Derzeit hängt ein kompetenter Einsatz digitaler Medien zu sehr am persönlichen Engagement und der Motivation einzelner Lehr- und Ausbildungskräfte, wie die beschriebenen Studien zeigen. Die Notwendigkeit zur Schaffung von Unterstützungs- und Beratungsstrukturen für das pädagogische Personal in der Berufsbildung zum Umgang mit digitalen Medien ist deutlich sichtbar.

- Auch die mangelnde bzw. nicht geprüfte Qualität oder die begrenzte Verbreitung von speziellen Lernprogrammen sowie die fehlende Entwicklung von Szenarien hemmt das Lehr- und Ausbildungspersonal beim Einsatz digitaler Medien (BIBB, 2018, S. 83 ff.; Schmid et al., 2016). Eine Qualitätsprüfung eingesetzter Lernanwendungen ist auch mit Blick auf die Studien aus Sicht des pädagogischen Personals notwendig. 
- In verschiedenen Studien wird mit der domänenspezifischen Forschung ein sehr zentraler Aspekt der digitalen Transformation aufgegriffen und berücksichtigt. Berufliche Domänen unterscheiden sich zum Teil stark voneinander, was zu einer zunehmenden Relevanz domänenspezifischer Forschung und Entwicklung, z. B. von fachdidaktischen Szenarien, führt.

- Die gesamte Bildungsorganisationsentwicklung mit dem wesentlichen Baustein der Aus- und Fortbildung des Lehr- und Ausbildungspersonals wird bisher in der Berufsbildungsforschung wenig beachtet, was sich auch in der nur geringfügig thematisierten institutionellen Verankerung digitaler Lernanwendungen und von Medientechniken zeigt. Wenig Beachtung geschenkt wird dem hohen Aufwand bei der Gestaltung eines digitalisierten Schulalltags in den berufsbildenden Schulen mit ihren sehr vielseitig angelegten Bildungsgängen für Schüler und Schülerinnen mit unterschiedlichen Bildungsvoraussetzungen. Auch die Potenziale eines kompetenten Einsatzes digitaler Medien im Unterricht bei der Förderung unterschiedlich benachteiligter Schüler und Schülerinnen, beispielsweise in der Ausbildungsvorbereitung, werden kaum thematisiert (Schmid et al., 2016).

Abschließend lässt sich konstatieren, dass es bislang offenbar nicht (ausreichend) gelingt, bereits bestehende Forschungsergebnisse in die Ausbildungspraxis zu transferieren. Um der Geschwindigkeit der digitalen Transformation gerecht zu werden, reicht es nicht aus, einfach nur Transparenz über bestehende Erkenntnisse der Forschung herzustellen. Vielmehr gilt es, den Dialog zwischen Wissenschaft und Praxis zu verstärken um wechselseitig Entwicklungen voran zu treiben.

\section{Literatur}

* durch Reviewprozess ausgewählte Studie

Bach, A. (2016a). Medienkompetenzentwicklung als Zielperspektive beruflicher Bildung im Zeitalter von Industrie 4.0. Die berufsbildende Schule, 68(9), 302-307. URN: urn:nbn: de:0111-pedocs-156206

Bach, A. (2016b). Nutzung von digitalen Medien an berufsbildenden Schulen - Notwendigkeit, Rahmenbedingungen, Akzeptanz und Wirkungen. In J. Seifried, U. Faßhauer \& S. Seeber (Hrsg.), Jahrbuch der berufs- und wirtschaftspädagogischen Forschung 2016 (S. 107-123). Opladen: Budrich.

Baumert, J., Kunter, M. (2006). Stichwort: Professionelle Kompetenz von Lehrkräften. Zeitschrift für Erziehungswissenschaft, 9(4), 469-520.

Baumgartner, P., Brandhofer, G., Ebner, M., Gradinger, P. \& Korte, M. (2016). Medienkompetenz fördern - Lehren und Lernen im digitalen Zeitalter. Die Österreichische Volkshochschule. Magazin für Erwachsenenbildung, 67(259), 3-9.

Blömeke, S. (2000). Medienpädagogische Kompetenz. Theoretische und empirische Fundierung eines zentralen Elements der Lehrerausbildung. München: KoPäd Verlag. 
Blömeke, S. (2003). Neue Medien in der Lehrerausbildung. $\mathrm{Zu}$ angemessenen (und unangemessenen) Zielen und Inhalten des Lehramtsstudiums. MedienPädagogik (Occasional Papers), 1-29.

Breiter, A., Howe, F. \& Härtel, M. (2018). Medien- und IT-Kompetenz des betrieblichen Ausbildungspersonals. Berufsbildung in Wissenschaft und Praxis, (3), 24-29.

${ }^{*}$ Bundesinstitut für Berufsbildung (BIBB). (2014). Ergebnisse der Online-Befragung zur Nutzung von "Foraus.de“ und zum Einsatz digitaler Medien in der dualen Berufsausbildung. Verfügbar unter: https://www.foraus.de/dokumente/media/Ergebnisse_ Umfrage_foraus.de.pdf

Bundesinstitut für Berufsbildung (BIBB). (2018). Ausbildung gestalten. Kaufmann im E-Commerce/Kauffrau im E-Commerce. Verfügbar unter: https://www.bibb.de/de/berufeinfo.php/profile/apprenticeship/261016

${ }^{\star}$ Bundesministerium für Bildung und Forschung (BMBF). (2018/2019). MESA - Medieneinsatz in der Schweißausbildung. Abschlussbericht. Berlin. Verfügbar unter: http:// mesa.ikap.biba.uni-bremen.de/wp-content/uploads/2019/04/MESA-Abschluss bericht. pdf

Bundesministerium für Bildung und Forschung (BMBF) (Hrsg.). (2019). e-Qualification. Lernen und Beruf digital verbinden. Projektband des Förderbereiches „Digitale Medien in der beruflichen Bildung“. Berlin. Verfügbar unter: https://www. qualifizierungdigital.de/_medien/downloads/eQualification_Projektband\%20A 5_2019_Web_(BITV)_Impressum-323.pdf

Carretero, S., Vuorikari, R. \& Punie, Y. (2017). DigComp 2.1: The Digital Competence Framework for Citizens with eight proficiency levels and examples of use. Luxembourg: European Union.

${ }^{*}$ Conrad, M. \& Schumann, St. (2017). Lust und Frust im Tablet-PC-basierten Wirtschaftsunterricht. Befunde einer Interventionsstudie zur Erfassung des affektiven Unterrichtserlebens mittels Continous-State-Sampling. Zeitschrift für Berufs- und Wirtschaftspädagogik, 113, 2017/1, 33-55.

${ }^{\star}$ Conrad, M., Wiest, S. \& Schumann, St. (2015). Webbasiertes informelles Lernen im Wirtschaftsunterricht. In G. Niedermair (Hrsg.), Informelles Lernen. Annäherungen Problemlagen - Befunde (Schriftenreihe für Berufs- und Betriebspädagogik, Band 9, S. 251-264). Linz: Trauner.

*DGB-Bundesvorstand. (2019). Ausbildungsreport 2019. Themenschwerpunkt: Ausbildung 4.0. Berlin. Verfügbar unter: https://www.dgb.de/themen/++co++9cafb4fe-c996-11e9b8a9-52540088cada

Ferrari, A. (2012). Digital Competence in Practice: An Analysis of Frameworks. Technical Report by the Joint Research Centre of the European Commission, Institute for Prospective Technological Studies. Online verfügbar unter: https://www.ifap.ru/library/ book522.pdf

Grant, M. J. \& Booth, A. (2009). A typology of reviews. An analysis of 14 review types and associated methodologies. Health information and libraries journal, 26(2), 91-108. https://doi.org/10.1111/j.1471-1842.2009.00848.x

Hall, A., Maier, T., Helmrich, R. \& Zika, G. (2016). IT-Berufe und IT-Kompetenzen in der Industrie 4.0. Bonn: Bundesinstitut für Berufsbildung.

Härtel, M., Averbeck, I., Brüggemann, M., Breiter, A., Howe, F. \& Sander, M. (2018a). Medien- und IT-Kompetenz als Eingangsvoraussetzung für die berufliche Ausbildung Synopse. Bonn: Bundesinstitut für Berufsbildung. Verfügbar unter: https://www.bibb.de/ veroeffentlichungen/de/publication/download/9223

${ }^{\star}$ Härtel, M., Brüggemann, M., Sander, M., Breiter, A., Howe, F. \& Kupfer, F. (2018b). Digitale Medien in der betrieblichen Berufsausbildung. Medienaneignung und Mediennutzung 
in der Alltagspraxis von betrieblichem Ausbildungspersonal. Bonn: Bundesinstitut für Berufsbildung. Verfügbar unter https://www.bibb.de/veroeffentlichungen/de/publica tion/download/9412.

Hirsch-Kreinsen, H., Ittermann, P. \& Niehaus, J. (Hrsg.). (2015). Digitalisierung industrieller Arbeit. Die Vision Industrie 40.0 und ihre sozialen Herausforderungen. Baden-Baden: Nomos. https://doi.org/10.5771/9783845283340

*Institut für Qualitätsentwicklung an Schulen Schleswig-Holstein (IQSH). (2018). Landesweite Umfrage zur IT-Ausstattung und Medienbildung der Schulen in SchleswigHolstein. Kronshagen. Verfügbar unter: https://www.schleswig-holstein.de/DE/Landes regierung/IQSH/Publikationen/PDFDownloads/ITMedien/Downloads/itUmfrage2018. pdf?_blob=publicationFile\&v $=1$

*Jablonka, P., Kröll, J. \& Metje, U. M. (2018). Ausgestaltung der Berufsausbildung und Handeln des Bildungspersonals an den Lernorten des dualen Systems. Ergebnisse betrieblicher Fallstudien. Berlin, Bundesinstitut für Berufsbildung. Verfügbar unter: https:// www.foraus.de/dokumente/pdf/Endbericht_Gestaltung_betrieblicher_Ausbildung_ Maerz_2018.pdf

Koehler, M. \& Mishra, P. (2009). What Is Technological Pedagogical Content Knowledge? Contemporary Issues in Technology and Teacher Education (CITE Journal), 9(1), 60-70.

Köhler, T. \& Niethammer, M. (2018). Digitale Unterstützung des Lernens. Berufsbildung, $72(171), 3-6$.

${ }^{\star}$ Kreijns, K., Vermeulen, M., Van Acker, F. \& Van Buuren, H. (2014). Predicting teachers' use of digital learning materials: combining selfdetermination theory and the integrative model of behavior prediction. European Journal of Teacher Education. DOI: $10.1080 / 02619768.2014 .882308$

Kultusministerkonferenz. (2017). Bildung in der digitalen Welt. Strategie der Kultusministerkonferenz. Beschluss der Kultusministerkonferenz vom 08.12.2016 in der Fassung vom 07.12.2017. Verfügbar unter: https://www.kmk.org/fileadmin/Dateien/veroeffentlichungen_beschluesse/2018/Strategie_Bildung_in_der_digitalen_Welt_idF._vom_07.12.2017. pdf

Kussau, J. \& Brüsemeister, T. (2007). Educational Governance. Zur Analyse der Handlungskoordination im Mehrebenensystem der Schule. In H. Altrichter, T. Brüsemeister \& J. Wissinger (Hrsg.), Educational Governance. Handlungskoordination und Steuerung im Bildungssystem (S. 15-54). Wiesbaden: Springer VS.

Müller-Böling, D. \& Müller, M. (1986). Akzeptanzfaktoren der Bürokommunikation. München, Wien: Oldenburg.

Scheer, A.-W. \& Wachter, C. (2016). Digitale Bildungslandschaften. Saarbrücken: imc information multimedia communication AG.

${ }^{\star}$ Schmid, U., Goertz, L. \& Behrens, J. (2016). Monitor Digitale Bildung. Berufliche Ausbildung im digitalen Zeitalter. BertelsmannStiftung. Verfügbar unter: https://www.bertelsmannstiftung.de/fileadmin/files/BSt/Publikationen/GrauePublikationen/Studie_MonitorDigitale-Bildung_Berufliche-Ausbildung-im-digitalen-Zeitalter_IFT_2016.pdf

${ }^{\star}$ Seufert, S., Guggemos, J., Tarantini, E. \& Schumann, St. (2019). Professionelle Kompetenzen von Lehrpersonen im Kontext des digitalen Wandels. Entwicklung eines Rahmenkonzepts und Validierung in der kaufmännischen Domäne. Zeitschrift für Berufs- und Wirtschaftspädagogik, 115(2), 312-339.

${ }^{\star}$ Sloane, P. F. E., Emmler, T., Gössling, B., Hagemeier, D., Hegemann, A. \& Janssen, E. A. (2018). Berufsbildung 4.0 - Qualifizierung des pädagogischen Personals als Erfolgsfaktor beruflicher Bildung in der digitalisierten Arbeitswelt. Wirtschaftspäd. Forum Bd. 63. Detmold. 
Tulodziecki, G., Herzig, B. \& Grafe, S. (2010). Medienbildung in Schule und Unterricht. Grundlagen und Beispiele. Bad Heilbrunn: UTB.

*Valtonen, T., Kontkanen, S., Dillon, P., Kukkonen, J. \& Väisänen, P. (2013). Upper secondary and vocational level teachers at social software. Educ Inf Technol, 19, 763-779. DOI 10.1007/s10639-013-9252-1

Venkatesh, V. \& Davis, F. D. (2000). A Theoretical Extension of Technology Acceptance Model: Four Longitudinal Field Studies. Management Science, 46(2), 186-204. Verfügbar unter: https://pubsonline.informs.org/doi/pdf/10.1287/mnsc.46.2.186.11926

*Virnes, M., Thiele, J., Manhart, M. \& Thalmann, St. (2017). Application Scenarios of Mobile Learning in Vocational Training: A Case Study of Ach So! In the construction Sector. EdMedia 2017 - Washington, DC, United States, June 20-23, 2017. Verfügbar unter: https://www.learntechlib.org/p/178308

Vollmer, T., Jaschke, St. \& Schwenger, U. (Hrsg.). (2017). Digitale Vernetzung der Facharbeit Gewerblich-technische Berufsbildung in einer Arbeitswelt des Internets der Dinge. Bielefeld: W. Bertelsmann Verlag. 
Jan Koschorreck \& Angelika Gundermann

\title{
Die Implikationen der Digitalisierung für das Lehrpersonal in der Erwachsenen- und Weiterbildung
}

\author{
Ein Review ausgewählter empirischer Ergebnisse und weiterer \\ theoriebildender Literatur
}

\begin{abstract}
Dieses Critical Review basiert auf einer Synthese aus 41 größtenteils internationalen empirischen Studien sowie auf kontextbildender Literatur aus Forschung und Praxis aus den Jahren 2016 bis 2019. Es bietet einen Überblick zu Implikationen der Digitalisierung für das Lehrpersonal in der Erwachsenen- und Weiterbildung (nachfolgend: EB/WB) in Bezug auf (1) Kompetenzen, (2) Haltung bzw. Rolle (3) Aus- und Fortbildung sowie (4) relevante Trends. Es zeigt sich, dass die Digitalisierung hohe Anforderungen an die Kompetenzen der Lehrenden in den Bereichen Didaktik, Methodik, Kommunikation, Interaktion und Organisation stellt. Es existieren fundierte Kompetenzmodelle, die sich für die systematische Erfassung der digitalen Kompetenzen eignen, aber noch entsprechend operationalisiert und gegebenenfalls empirisch getestet werden müssen. Die professionelle Haltung von Lehrenden im Umgang mit digitalen Technologien und der zu beobachtende Rollenwandel vom Wissensvermittelnden hin zum Lernbegleitenden insbesondere auch in digitalen Lernformaten sind zentrale Elemente bei der Digitalisierung in der EB/WB. Die Aneignung digitaler Kompetenzen verläuft vor allem informell bzw. im Selbststudium und dementsprechend heterogen. Geeignete Fortbildungsmaßnahmen sollten effizient und praxisnah gestaltet und durch Peers oder die eigene Institution unterstützt werden. Die Themen Kompetenzen, Aus- und Fortbildung sowie der Rollenwandel von Lehrenden im Rahmen der Digitalisierung bleiben auch perspektivisch relevant. Abschließend werden auf Basis des Reviews Implikationen für Forschung, Politik und Bildungspraxis diskutiert.
\end{abstract}

Schlüsselwörter: Erwachsenenbildung, Weiterbildung, Critical Review, Lehrkräfte, Kompetenzen, Rolle

\section{Implications of digitisation on teachers and trainers in adult and continuing education and training}

This critical review is based on a synthesis of 41 mostly international empirical studies as well as context literature published between 2016 and 2019. It provides an overview of the implications digitisation has on teachers and trainers in adult and continuing education and training in terms of (1) competences, (2) roles and attitudes, (3) continuing education and training, (4) relevant future trends. Digitisation places high demands on the teachers' competences regarding didactics, methodology, communication, interaction, and organisation. There are well-grounded competence models that are appropriate to systematically map digital competences, but their operationalisation and empirical testing are still lacking. The professional attitudes of teachers and trainers in adult and continuing 
education and training towards digital technologies and the perceivable change of their role from conveyor of knowledge to facilitator/curator especially in digital environments are crucial for the process of digitisation. Teachers and trainers acquire digital competences informally and thus heterogeneously. Appropriate courses need to be designed efficiently and aligned to teaching practice, supported by peers or the connected institution. Competences, further education and training as well as the changing roles of teachers and trainers within the scope of digitisation will remain relevant issues. Further implications for policy and the practice of adult and continuing education and training are discussed based on the findings.

Keywords: adult education, continuing education, training, critical review, trainers, teachers, competences, role, attitude

\section{Einleitung}

Die EB/WB ist mit Blick auf die Menge (potenzieller) Adressaten und Adressatinnen, die Zahl der Veranstaltungen und die der Anbieter der größte Bildungsbereich in Deutschland (Faulstich \& Zeuner, 1999; Schrader \& Loreit, 2018). Im Vergleich zur Schule oder Hochschule ist der Bereich unterfinanziert, die Förderung eher rückläufig (Dollhausen, 2008; Schrader, 2019). Die Struktur der Anbieter im Feld ist sehr vielfältig; sie reicht von staatlich bzw. gemeinschaftsorientierten Trägern wie den Volkshochschulen oder konfessionellen Bildungseinrichtungen über marktorientierte Anbieter von Weiterbildung wie beispielsweise Sprachlerninstitute bis hin zu unternehmenseigenen bzw. innerbetrieblichen Bildungseinrichtungen (Kraft, 2018; Schrader, 2011, 2019). Ähnliches gilt für die Beschäftigtenstruktur im Bereich Weiterbildung: Zum einen arbeitet hier eine eher geringe Zahl von unbefristet beschäftigten, hauptamtlichen pädagogischen Mitarbeiterinnen und Mitarbeitern. Sie sind unter Umständen anteilig in der Lehre tätig, ihre Aufgaben liegen aber hauptsächlich im Bereich Leitung, Bildungsadministration und Programmplanung (Autorengruppe wb-personalmonitor, 2016, S. 102). Der überwältigende Anteil der Lehre wird von mindestens 530.000 neben- bzw. freiberuflich beschäftigten Personen erbracht. Ihre wirtschaftliche Situation ist z. T. prekär: Mehr als die Hälfte der Honorarkräfte und Selbstständigen erwirtschaftete 2014 weniger als 1700 Euro Bruttoeinkommen im Monat (ebd., S. 87). Zwar verfügen 66,6\% der Lehrenden über einen akademischen Abschluss, es haben jedoch nur 26,3\% der Lehrenden ein explizit pädagogisches oder erziehungswissenschaftliches Studium absolviert (ebd., S. 111). Die Geschlechterverteilung innerhalb der Gruppe der Lehrenden ist mit 50,1\% zu 49,9\% nahezu ausgeglichen. Das Durchschnittsalter aller in der EB/WB tätigen Personen liegt bei 50,5 Jahren (ebd., S. 63, S. 68). Im Diskurs zur Digitalisierung von Weiterbildung stehen in der Praxis insbesondere Potenziale digitaler Technologien im Mittelpunkt, aber auch damit verbundene Anforderungen und Handlungsdruck (z. B. Schöll, 2017). Digitale Technologien können Lehrenden als Medium der Vermittlung wie auch als kreative Werkzeuge im Lernprozess dienen 
(vgl. Diethelm, 2018, S. 38f.). Beachtung finden digitale Technologien auch aufgrund ihrer Möglichkeiten, Lernangebote und -erfolge zu verbessern und neue Zielgruppen $\mathrm{zu}$ erreichen bzw. bestehende Zielgruppen noch passgenauer anzusprechen. Lehrende bestimmen maßgeblich den Erfolg oder Misserfolg von Lernangeboten mit und sind zentrale Akteure im Bemühen, das Potenzial digitaler Technologien für die Erwachsenenbildung zu realisieren (Mayert, 2018, S. 15f.; Rohs \& Weber, 2018, S. 18; Schrader, 2010, S. 30 f.). Daher ist es sinnvoll, nach Implikationen der Digitalisierung für die Lehrenden in der EB/WB zu fragen. Das Thema wird nachfolgend unter verschiedenen Teilaspekten betrachtet, die in diesem Sammelband als bereichsübergreifend relevant für die Fragestellung identifiziert wurden: (1) in Bezug auf die Kompetenzen sowie (2) die Haltung bzw. Rolle von Lehrenden, (3) hinsichtlich ihrer Aus- und Fortbildung und (4) mit Blick auf für Lehrende relevante Entwicklungen.

\section{Methode}

Die Erarbeitung erfolgte nach der Methode des Critical Reviews (Grant \& Booth, 2009), die sich im Vorgehen an Systematic Reviews orientiert, um auf der Basis einer umfangreichen Literaturrecherche Informationen über ein zuvor definiertes Thema zu liefern. Ziel ist es, einen konzeptionellen Beitrag zur Erfassung eines Themas zu leisten (Wilmers, Anda, Keller, Kerres \& Getto im gleichen Band; Grant \& Booth, 2009, S. 93 ff.).

Im Zuge der in diesem Sammelband zunächst für alle Bildungssektoren gleichartig konzipierten Literaturrecherche (Wilmers et al., S. $18 \mathrm{ff}$.) wurden die Wortgruppen für die bildungsbereichsspezifische Suche mit entsprechenden Begriffen ergänzt bzw. angepasst. So wurden beispielsweise in der Wortgruppe Pädagogisches Personal die Begriffe Lehrende, Trainer und Trainerin, Dozent und Dozentin, Erwachsenenbildner und Erwachsenenbildnerin, Kursleiter und Kursleiterin verwendet, da der Begriff Lehrer bzw. Lehrerin in der EW/WB nicht gebräuchlich ist.

Die Literaturrecherche ergab für den Zeitraum 2010 bis 2018 insgesamt 2185 Treffer mit durchschnittlich 239 Veröffentlichungen pro Jahr, die meisten für das Jahr 2017 mit 277 Titeln, gefolgt von 2016 mit 272, und den wenigsten für das Jahr 2014 mit 210 Titeln. 2018 erschienen mit 256 wiederum weniger Titel als in den Vorjahren, so dass sich hier kein Trend zu verstärkter Publikationstätigkeit in jüngster Zeit ablesen lässt. Angesichts dieser großen Zahl an Literaturfunden entschieden sich die Autoren für die Bearbeitung der Veröffentlichungen ab 2016, um vor allem aktuellere Dynamiken in den Blick zu nehmen. Damit wurden 805 Titel zunächst anhand der Abstracts und Schlagworte auf Passung zum Bildungsbereich und der Fragestellung geprüft. Bei diesem ersten Screening der Literaturfunde erwies es sich außerdem als notwendig, eine Abgrenzung $\mathrm{zu}$ den Bereichen berufliche Bildung und Hochschulbildung zu definieren. Texte mit Bezug zu beruf- 
licher Erstausbildung, zu dualer Ausbildung bzw. dualem Studium - im Sinne von Erstausbildung sowie geregelte Fortbildungen wie z.B. Meisterausbildung im Handwerk - wurden ausgeschlossen. Texte zur Hochschulbildung wurden nur rezipiert, wenn es sich um Übersichtsstudien (Metastudien oder Literaturreviews) handelte oder sie die Fernlehre, wissenschaftliche Weiterbildung und Studiengänge der Erwachsenenbildung thematisierten.

Zentrale Entscheidungskriterien für die Berücksichtigung von Texten waren die Validität des Vorgehens, die Reliabilität bzw. Plausibilität der Studie sowie deren Generalisierbarkeit (Booth, Sutton \& Papaioannou, 2016, S. 151-155) - für den Zweck dieses Reviews verstanden als Übertragbarkeit der Ergebnisse auf die EB/ WB im deutschsprachigen Raum bzw. die Anwendbarkeit auf Lehrende in diesem Bereich. Fehlender Bezug auf die Fragestellung und mangelnde Übertragbarkeit, zum Beispiel durch extrem spezifische Ausgangslagen und/oder länderspezifische Konstellationen, führten zum Ausschluss. Nach diesem Verfahren verblieben insgesamt 394 Publikationen. Diese wurden im Volltext geprüft und nach Art der Studie sortiert, inhaltlich zusammengefasst und kodiert und schließlich analysiert sowie hinsichtlich ihrer Qualität und ihrer Eignung für den Bericht bewertet.

Die durch diesen Prozess ermittelten berichtsrelevanten Publikationen wurden gemäß den festgelegten Teilaspekten kategorisiert (s. Wilmers et al. in diesem Band, S. 8). Parallel erfolgten eine Erfassung, Verschlagwortung und Zuordnung relevanter Textstellen. Mittels kommunikativer Validierung der Textstellen wurden diese in einem weiteren Schritt überprüft und thematisch geordnet, um verschiedene Aspekte innerhalb der Teilfragestellungen aufzudecken und eine Vorstrukturierung der vorliegenden Synthese zu erreichen. Schlussendlich fanden im vorliegenden Review insgesamt 57 Publikationen Berücksichtigung, 18 Titel theoriebildende Literatur und 41 empirische Studien (Tab. 1 und 2). 
Tabelle 1: Theorie- bzw. Kontextbildende Literatur

\begin{tabular}{|c|c|c|c|c|c|c|}
\hline \multirow[t]{2}{*}{ Autorlnnen } & \multirow[t]{2}{*}{ Jahr } & \multirow[t]{2}{*}{$\begin{array}{l}\text { Geogr. } \\
\text { Bezug }\end{array}$} & \multicolumn{4}{|c|}{$\begin{array}{l}\text { Bezug zu Teilaspekten der } \\
\text { Fragestellung }\end{array}$} \\
\hline & & & 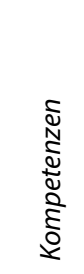 & 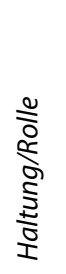 & 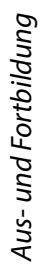 & 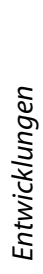 \\
\hline Arnold, R., Nuissl, E. \& Rohs, M. & 2017 & $\mathrm{DE}$ & & & & $x$ \\
\hline Bolten, R. & 2018 & DE & $x$ & & & \\
\hline Bremer, C. & 2017 & $\mathrm{DE}$ & $x$ & & & $x$ \\
\hline Helbig, C. \& Hofhues, S. & 2018 & $\mathrm{DE}$ & $x$ & & & \\
\hline Kühn, C., Robak, S. \& Fleige, M. & 2018 & $\mathrm{DE}$ & & & & $x$ \\
\hline Mayert, A. & 2018 & $\mathrm{DE}$ & $\mathrm{x}$ & & $x$ & $x$ \\
\hline Mayrberger, K. & 2017 & $\mathrm{DE}$ & & $x$ & & \\
\hline Niedermeier, S. \& Müller, C. & 2016 & $\mathrm{DE}$ & & & & $x$ \\
\hline Pachner, A. & 2018 & DE & $x$ & & & \\
\hline Richter, T. & 2017 & DE & & & $x$ & \\
\hline Rohs, M. \& Weber, C. & 2018 & $\mathrm{DE}$ & & & $x$ & \\
\hline Rossmann, E. D. & 2016 & $\mathrm{DE}$ & & & & $x$ \\
\hline Sgier, I., Haberzeth, E. \& Schüepp, P. & 2018 & $\mathrm{CH}$ & $\mathrm{x}$ & & $x$ & \\
\hline Staudacher, N. & 2019 & DE & & & & $x$ \\
\hline Umbach, S., Böving, H. \& Haberzeth, E. & 2018 & DE & & & & $\mathrm{x}$ \\
\hline Wiemeyer, J. & 2016 & $\mathrm{DE}$ & $\mathrm{x}$ & & & $x$ \\
\hline Wienberg, J., Heinrichs, H. S. \& Grotlüschen, A. & 2018 & $\mathrm{DE}$ & $x$ & $x$ & & \\
\hline Wildemeersch, D. \& Jütte, W. & 2017 & EU & & & & $x$ \\
\hline
\end{tabular}


Tabelle 2: Empirische Studien und Studien zu Modellentwicklungen

\section{Autorlnnen}

\section{Jahr Sample Art der Geograf. Unterkapitel n Studie Bezug}

\begin{tabular}{|c|c|c|c|c|c|c|c|c|}
\hline & & & & & 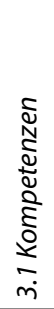 & 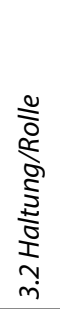 & 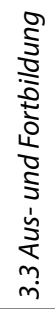 & 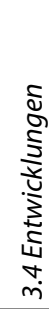 \\
\hline $\begin{array}{l}\text { Adams Becker, S., Brown, M., Dahlstrom, } \\
\text { E., Davis, A., DePaul, K., Diaz, V. \& Pomer- } \\
\text { antz, J. }\end{array}$ & 2018 & 71 & * & $\begin{array}{l}\text { interna- } \\
\text { tional }\end{array}$ & & & & $\mathrm{x}$ \\
\hline $\begin{array}{l}\text { Adams Becker, S., Cummins, M., Davis, A., } \\
\text { Freeman, A., Hall Giesinger, C. \& Anan- } \\
\text { thanarayanan, V. }\end{array}$ & 2017 & 78 & * & $\begin{array}{l}\text { interna- } \\
\text { tional }\end{array}$ & & & & $\mathrm{x}$ \\
\hline $\begin{array}{l}\text { Alexander, B., Ashford-Rowe, K., Barajas- } \\
\text { Murphy, N., Dobbin, G., Knott, J., McCor- } \\
\text { mack, M., Pomerantz, J., Seilhamer, R. \& } \\
\text { Weber, N. }\end{array}$ & 2019 & 98 & * & $\begin{array}{l}\text { interna- } \\
\text { tional }\end{array}$ & & & & $x$ \\
\hline Autorengruppe wb-Personalmonitor & 2016 & 6000 & $\%$ & $\mathrm{DE}$ & & & $\mathrm{x}$ & \\
\hline Bolten, R., Kohl, J. \& Rohs, M. & 2018 & 47 & $\%$ & $\mathrm{DE}$ & $\mathrm{x}$ & & & \\
\hline $\begin{array}{l}\text { Bolten, R., Rohs, M., Schmidt-Hertha, B. \& } \\
\text { Rott, K. J. }\end{array}$ & 2019 & $622^{\#}$ & $\%$ & $\mathrm{DE}$ & $\mathrm{x}$ & & & \\
\hline Bolten, R. \& Rott, K. & 2018 & $4 / 5$ & * & $\mathrm{DE}$ & $\mathrm{x}$ & $x$ & & \\
\hline Burchert, J. \& Grobe, R. & 2017 & 14 & * & $\mathrm{DE}$ & & $x$ & & \\
\hline Caena, F. \& Redecker, C. & 2019 & n.z. & M & EU & $x$ & & & \\
\hline Cendon, E. & 2018 & $26 / 7$ & * & $\mathrm{DE}$ & & $x$ & & \\
\hline Colliander, $\mathrm{H}$. & 2019 & 13 & ${ }^{*}$ & $\mathrm{EU}$ & & $\mathrm{x}$ & & \\
\hline $\begin{array}{l}\text { Ferguson, R., Herodotou, C., Coughlan, T., } \\
\text { Scanlon, E. \& Sharples, M. }\end{array}$ & 2018 & 167 & $\mathrm{R}$ & $\mathrm{EU}$ & $\mathrm{x}$ & & & \\
\hline Ghomi, M. \& Redecker, C. & 2019 & 335 & $\%$ & $\begin{array}{l}\text { interna- } \\
\text { tional }\end{array}$ & $\mathrm{x}$ & & & \\
\hline Kennedy, I. G., Latham, G. \& Jacinto, H. & 2016 & 200 & * & $\begin{array}{l}\text { interna- } \\
\text { tional }\end{array}$ & $\mathrm{x}$ & & & \\
\hline Klauser, F. \& Schlicht, J. & 2016 & $18 / 10$ & $\%$ & $\mathrm{DE}$ & & & $x$ & \\
\hline $\begin{array}{l}\text { Laurian-Fitzgerald, S., Fitzgerald, C. J., } \\
\text { Popa, C. A. \& Bochis, L. }\end{array}$ & 2018 & 241 & $\%$ & USA & $x$ & & & \\
\hline Littlejohn, A. \& Hood, N. & 2017 & $\begin{array}{c}521 \\
(468)\end{array}$ & * & EU & & $\mathrm{x}$ & & \\
\hline $\begin{array}{l}\text { Loizzo, J., Ertmer, P. A., Watson, W. R. \& } \\
\text { Watson, S. L. }\end{array}$ & 2017 & $671 / 12$ & $\% / *$ & US & $x$ & & & \\
\hline $\begin{array}{l}\text { Mavropoulos, A. A., Sipitanou, A. K. \& } \\
\text { Pampouri, A. A. }\end{array}$ & 2019 & 222 & $\%$ & GR & & & $\mathrm{x}$ & \\
\hline mmb Institut (Hrsg.) & 2016 & 68 & * & $\mathrm{DE}$ & & & & $\mathrm{x}$ \\
\hline mmb-Trendmonitor I/2017 & 2017 & 74 & $*$ & $\begin{array}{l}\mathrm{DE}, \mathrm{AT}, \\
\mathrm{CH}\end{array}$ & & & & $x$ \\
\hline
\end{tabular}




\begin{tabular}{|c|c|c|c|c|c|c|c|c|}
\hline & & & & & 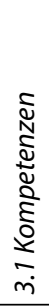 & 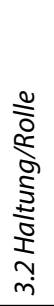 & 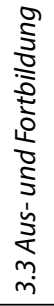 & 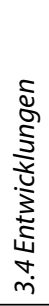 \\
\hline mmb Trendmonitor $2017 / 2018$ & 2018 & 61 & * & $\begin{array}{l}\mathrm{DE}, \mathrm{AT} \\
\mathrm{CH}\end{array}$ & & & & $\mathrm{x}$ \\
\hline mmb Trendmonitor 2018/2019 & 2019 & 65 & * & $\begin{array}{l}\mathrm{DE}, \mathrm{AT} \\
\mathrm{CH}\end{array}$ & & & & $x$ \\
\hline Montebello, M. & 2017 & 80 & * & EU & & $\mathrm{x}$ & & \\
\hline $\begin{array}{l}\text { Nafukho, F. M., Alfred, M., Chakraborty, } \\
\text { M., Johnson, M. \& Cherrstrom, C. A. }\end{array}$ & 2017 & 251 & $\%$ & USA & & & $\mathrm{x}$ & \\
\hline Paepe, L. de, Zhu, C. \& Depryck, K. & 2018 & $13^{\# \#}$ & * & EU & $\mathrm{x}$ & & & \\
\hline Paepe, L. de, Zhu, C. \& Depryck, K. & 2019 & $13^{\# \#}$ & * & EU & & $\mathrm{x}$ & & \\
\hline $\begin{array}{l}\text { Pérez-Paredes, P., Ordoñana Guillamón, C. } \\
\text { \& Aguado Jiménez, P. }\end{array}$ & 2018 & 230 & $\%$ & UK, ES & & & $\mathrm{x}$ & \\
\hline Redecker, C. \& Punie, Y. & 2017 & n. z. & M & EU & $\mathrm{x}$ & & & \\
\hline Roberts, J. & 2018 & 119 & $\%$ & $\begin{array}{l}\text { interna- } \\
\text { tional }\end{array}$ & & $\mathrm{x}$ & & \\
\hline Rohs, M. \& Bolten, R. & 2017 & 5 & * & $\mathrm{DE}$ & & & $\mathrm{x}$ & \\
\hline Rohs, M., Bolten, R. \& Kohl, J. & 2017 & 12 & * & $\begin{array}{l}\text { interna- } \\
\text { tional }\end{array}$ & $x$ & & & \\
\hline $\begin{array}{l}\text { Rohs, M., Rott, K. J., Schmidt-Hertha, B. \& } \\
\text { Bolten, R. }\end{array}$ & 2017 & n. $z$. & M & $\mathrm{DE}$ & $x$ & & & \\
\hline Rott, K. J. \& Schmidt-Hertha, B. & 2019 & $622^{\#}$ & $\%$ & $\mathrm{DE}$ & $\mathrm{x}$ & & & \\
\hline Schmid, U., Goertz, L. \& Behrens, J. & 2018 & $260 / 224$ & $\%$ & $\mathrm{DE}$ & $\mathrm{x}$ & & $\mathrm{x}$ & \\
\hline Schöb, S., Rohs, M., Biel, C. \& Scholze, T. & 2016 & 1367 & $\% / *$ & $\mathrm{DE}$ & & & $\mathrm{x}$ & \\
\hline Schrader, J. \& Schöb, S. & 2016 & 50 & $E$ & $\mathrm{DE}$ & & & $\mathrm{x}$ & \\
\hline Staab, M. \& Egetenmeyer, R. & 2019 & 166 & $\% / *$ & EU & & & $\mathrm{x}$ & \\
\hline Strauch, A. \& Lencer, S. & 2016 & n. z. & $\mathrm{M}$ & $\mathrm{DE}$ & $x$ & & & \\
\hline $\begin{array}{l}\text { Strauch, A., Lencer, S., Bosche, B., } \\
\text { Gladkova, V., Schneider, M. \& Treviño- } \\
\text { Eberhard, D. }\end{array}$ & 2019 & n. z. & $\mathrm{M}$ & $\mathrm{DE}$ & $x$ & & & \\
\hline Tan, S. C. & 2018 & 3 & $\mathrm{R}$ & $\begin{array}{l}\text { interna- } \\
\text { tional }\end{array}$ & $x$ & & & \\
\hline
\end{tabular}

Quantitative Studie $=\%$

Qualitative Studie $=$ *

(Quasi-)Experimentelle Studie $=\mathrm{E}$

Review $=$ R

Modellentwicklung $=M$

\#, \#\# etc. kennzeichnen Studien, die sich auf denselben Datensatz beziehen 


\section{Ergebnisse der Literaturanalyse}

In den folgenden Absätzen werden zunächst Ergebnisse der Analyse der kontextbzw. theoriebildenden Literatur wiedergegeben, die sich auf die vier oben genannten Teilaspekte beziehen. In den einzelnen Unterkapiteln stehen dann Ergebnisse aus den für das Review ermittelten empirischen Studien zu den Teilaspekten im Fokus.

In der kontext- bzw. theoriebildenden Literatur ist weithin anerkannt, dass die Digitalisierung die Anforderungen an Kompetenzen mindestens verändert, wenn nicht neue Kompetenzen fordert (Mayert, 2018; Pachner, 2018; Sgier, Haberzeth \& Schüepp, 2018). Helbig und Hofhues (2018) kommen in ihrer Analyse des medienpädagogischen Diskurses zu dem Schluss, dass die Digitalisierung es notwendig macht, medienpädagogische Leitideen in der EB/WB aufzugreifen, und medienpädagogische Kompetenzen auf Seiten der Lehrenden erfordert. Auch in Auseinandersetzungen mit konkreten Technologien auf konzeptioneller Ebene wird immer wieder die Bedeutung der Kompetenz der Lehrenden für deren erfolgreichen Einsatz betont (z.B. Mobiles Lernen: Bremer, 2017; Game-Based-Learning: Wiemeyer, 2016). Zugleich wird ein Kompetenzdefizit von Lehrenden konstatiert (Mayert, 2018; Rohs, Rott, Schmidt-Hertha \& Bolten, 2017).

Die pädagogische Haltung als Konglomerat individueller Einstellungen, Werte und Überzeugungen ist eng mit dem professionellen Selbstverständnis von Lehrpersonen verbunden (vgl. Schwer \& Solzbacher, 2014) und hat bedeutenden Anteil an der professionellen Handlungskompetenz von Lehrenden in der Erwachsenenund Weiterbildung (Bosche, Schneider \& Strauch, 2018, S. 48). Bolten (2018, S. 105) geht davon aus, dass die Haltung von Lehrenden im Sinne eines medienbezogenen Habitus das professionelle Handeln mit Medien und den Erwerb von Medienkompetenz beeinflusst bzw. begrenzt. Die Rolle der Lehrenden und das Verhältnis zu Lernenden wurde in den letzten Jahren insbesondere in der EB/WB mit der Rezeption konstruktivistischer Theorien neu definiert (vgl. Nuissl von Rein, 2012, S. 89 ff.). Dies trifft auch für digitale Lernformate zu (Wienberg, Heinrichs \& Grotlüschen, 2018; Mayrberger, 2017).

Aufgrund neuer mediendidaktischer und technischer Herausforderungen werden entsprechende Fort- und Weiterbildungen als essentiell betrachtet (Mayert, 2018; Richter, 2017; Rohs \& Weber, 2018; Sgier et al., 2018). Pachner (2018, S. 19) verweist in Zusammenhang mit der Frage nach Kompetenzen außerdem auf nationale bzw. übernationale bildungspolitische Setzungen, die diese Entwicklungen inzwischen verstärkt aufgreifen und in Forderungen nach Kompetenzaufbau auf Seiten der Lernenden und somit auch auf Seiten der Lehrenden überführen. Rohs und Weber (2018, S. 18) stellen mit Blick auf die wissenschaftliche Weiterbildung fest, dass nur wenige Fortbildungen spezifisch für Lehrpersonen in diesem Bereich angeboten werden.

Hinsichtlich zukünftiger Entwicklungen betonen sowohl die Forschung als auch Akteure aus Verbänden die weitreichenden Auswirkungen der Digitalisierung 
auf die Bildung (Bolten, Kohl \& Rohs, 2018, S. 1; Umbach, Böving \& Haberzeth, 2018, S. 16). Damit verbunden sind Forderungen nach Neubewertungen (Kühn, Robak \& Fleige, 2018, S. 184), nach (staatlicher) Förderung (Mayert, 2018, S. 15 f.; Rossmann, 2016, S. 6) oder nach Professionalisierung des Personals in der EB/WB (Wildemeersch \& Jütte, 2017, S. 18). Arnold, Nuissl und Rohs (2017, S. $233 \mathrm{ff}$.) sprechen dem Sektor der beruflichen Weiterbildung eine Vorreiterrolle in der Digitalisierung zu und begründen dies mit der starken Digitalisierung des Arbeitsumfeldes in den Unternehmen. Bremer (2017, S. 104) zufolge gewinnen im Zuge der Verbreitung mobiler Endgeräte informelle mobile Lernszenarien an Bedeutung. Game-Based-Learning bzw. Gamification werden als Trend vor allem in der beruflichen Weiterbildung beobachtet (Niedermeier \& Müller, 2016; Staudacher, 2019; Wiemeyer, 2016).

\subsection{Implikationen der Digitalisierung für Kompetenzen von Lehrpersonal}

Insgesamt beziehen sich 17 Studien aus dem Korpus auf den Zusammenhang von Digitalisierung und Kompetenzen von Lehrenden. Drei davon nehmen den Kompetenzstand von Lehrenden in den Blick (Schmid, Goertz \& Behrens, 2018; Bolten, Rohs, Schmidt-Hertha \& Rott, 2019; Rott \& Schmidt-Hertha, 2019). Sechs weitere thematisieren die Veränderungen von Kompetenzanforderungen bzw. neue Anforderungen durch die Digitalisierung (Ferguson, Herodotou, Coughlan, Scanlon, \& Sharples, 2018; Laurian-Fitzgerald, Fitzgerald, Popa \& Bochis, 2018; Loizzo, Ertmer, Watson \& Watson, 2017; Kennedy, Latham \& Jacinto, 2016; Paepe, Zhu \& Depryck, 2019; Tan, 2018). Acht Studien stehen im Zusammenhang mit der Entwicklung verschiedener Kompetenzmodelle mit unterschiedlicher Spezifizität in Bezug auf digitale Kompetenzen (Bolten \& Rott, 2018; Caena \& Redecker, 2019; Ghomi \& Redecker, 2019; Redecker \& Punie, 2017; Rohs, Bolten \& Kohl, 2017; Rohs, Rott et al., 2017; Strauch \& Lencer, 2016; Strauch et al., 2019). In der hier analysierten Literatur verwenden die jeweiligen Autorinnen und Autoren unterschiedliche Kompetenzbegriffe. Eine umfassende Diskussion der Unterschiede ist in Bezug auf die Fragestellung weder sinnvoll noch leistbar. Nachfolgend wird der Begriff der Kompetenz deshalb überwiegend als Sammelbegriff verwendet und nur vereinzelt unter Bezugnahme auf die Literatur differenziert, sofern es mit Blick auf die Fragestellung relevant erscheint.

Die systematische Verknüpfung von Anforderungen der Digitalisierung mit Kompetenzen von Lehrenden legt die Erarbeitung von Modellen nahe. Kompetenzmodelle bieten Orientierung und sind Ausgangspunkt einer Operationalisierung für empirische Bestandsaufnahmen, die Erstellung von Curricula und letztlich für die Gestaltung von Aus- bzw. Fortbildungsmaßnahmen (Rohs, Bolten \& Kohl, 2017, S. 3). 
Eine Vorstufe von Modellen sind umfangreiche Auflistungen von Kompetenzanforderungen an Lehrende. Eine solche erzielt beispielsweise die Studie von Kennedy, Latham und Jacinto (2016): Sie werteten in einer explorativ-qualitativen Studie 200 Textbeiträge von - in unterschiedlichen pädagogischen Kontexten aktiven - Mitgliedern des kommerziellen Forschernetzwerks ResearchGate zur Frage „What skills must 21 st century teachers have to promote high quality learning?" inhaltsanalytisch aus. Sie identifizieren 23 Themen. In der Auswertung der Diskussion zeigten sich Schwerpunkte in den Bereichen Feedback an Teilnehmende, Ermöglichung von Teilhabe, Medienkompetenz von Lehrenden, Umgang mit Unsicherheit und Veränderung durch Lehrende, sowie Arbeitsmotivation.

Mit dem GRETA-Kompetenzmodell liegt ein allgemeines Kompetenzmodell für Lehrende in der EB/WB vor (Strauch \& Lencer, 2016; Strauch et al., 2019). Es orientiert sich an einschlägigen theoretischen Grundlagen und basiert auf bestehenden Modellen und handlungsbezogenen Anforderungen aus der Praxis. Das Modell unterscheidet zunächst vier übergeordnete Kompetenzaspekte: „Professionelle Selbststeuerung“, „Berufspraktisches Wissen und Können“, „Fach- und Feldspezifisches Wissen“ sowie „Professionelle Werthaltungen und Überzeugungen“. Diese werden in eine unterschiedliche Anzahl von Kompetenzbereichen differenziert, die wiederum in Kompetenzfacetten aufgeschlüsselt sind. In einzelnen Kompetenzfacetten wie beispielsweise „Lehr-/Lernmethoden und Konzepte/neue Medien“ oder „Rahmenbedingungen und (mediale) Lernumgebungen“ finden sich Bezüge zu Anforderungen der Digitalisierung, beispielsweise im Bereich Didaktik und Methoden. Das Modell wurde auf der Basis von 20 Experteninterviews mit leitendem und planendem Personal sowie mit Lehrenden im Rahmen von Workshops entwickelt und kommunikativ validiert (Strauch \& Lencer, 2016, S. 4f.).

Das 2017 vorgestellte Digital Competence Framework for Educators (DigCompEdu) leistet eine systematische Darstellung von allgemeinen und digitalen Kompetenzen von Lehrenden. Es wurde vom Joint Research Centre der Europäischen Union entwickelt und beansprucht Gültigkeit für die gesamte Bildungskette, somit auch für den Bereich der EB/WB (Redecker \& Punie, 2017). Das Modell differenziert 22 Kompetenzen, die sich auf sechs Bereiche („Berufliches Engagement“, „Digitale Ressourcen“, „Lehren und Lernen“, „Evaluation“, „Lernerorientierung“, „Förderung der digitalen Kompetenz der Lernenden“) verteilen. Die Kompetenzen werden auf sechs Niveaustufen (A1 bis C2) operationalisiert, die vom Europäischen Referenzrahmen für Sprachen übernommen wurden. DigCompEdu wurde auf Basis der Auswahl und Analyse von 32 (aus einer Grundgesamtheit von 50) existierenden Frameworks, Modellen, Richtlinien oder Instrumenten zur Entwicklung digitaler Kompetenzen entworfen und anhand einer daran anschließenden Reihe von Konsultationen mit Experten und Akteuren aus der Bildung validiert (Caena \& Redecker, 2019, S. 361 f.). Eine Operationalisierung bzw. empirische Überprüfung für den Bereich EB/WB liegt zum Zeitpunkt dieses Reviews nicht vor, allerdings existiert 
ein auf dem Modell basierendes Self-Assessment-Tool ${ }^{1}$, dass sich in einer Pilotstudie mit 335 Pädagogen und Pädagoginnen, vorwiegend aus dem Schulbereich, als grundsätzlich valide und reliabel erwiesen hat (Ghomi \& Redecker, 2019, 547f.).

In einer umfassenden Analyse des Inhalts von zwölf Kompetenzbeschreibungen aus dem Bereich Erwachsenenbildung kommen Rohs, Bolten und Kohl (2017, S. $45 \mathrm{f}$.) $\mathrm{zu}$ dem Schluss, dass sich in jeder der analysierten Beschreibungen unterschiedlich gewichtete Anteile von medienpädagogischen Kompetenzfacetten wiederfinden, die vor allem im Bereich der Mediendidaktik verortet sind. Dabei stellen sie fest, dass die Modelle aus dem internationalen Raum medienpädagogische Aspekte insgesamt stärker betonen als es bei Modellen aus dem deutschsprachigen Raum der Fall ist. Auf Basis dieser Analyse entwerfen Rohs, Rott, Schmidt-Hertha und Bolten (2017) ein medienpädagogisches Kompetenzmodell. Darin differenzieren sie das Konstrukt „Medienpädagogische Handlungskompetenz“ in vier Kompetenzfacetten vor dem Hintergrund einer allgemeinen Medienkompetenz: mediendidaktische Kompetenz, fachbezogene Medienkompetenz, medienbezogene Feldkompetenz und medienbezogene personale Kompetenzen. In einer daran anschließenden qualitativen Studie im Umfang von neun Interviews und zwei Fokusgruppen wurde das Modell validiert bzw. weiterentwickelt (Bolten \& Rott, 2018). Dabei bestätigen die beiden Autorinnen durch systematische inhaltsanalytische Auswertung, dass sich Anforderungen der Digitalisierung von EB/WB auf verschiedene Ebenen des pädagogischen Handelns beziehen und auf die verschiedenen Kompetenzfacetten des Modells rekurrieren.

Das oben referierte Kompetenzmodell von Rohs, Rott, Schmidt-Hertha und Bolten (2017) wurde in einer umfangreichen Pilotstudie mit 622 Lehrenden aus dem Bereich Erwachsenenbildung getestet (Bolten et al., 2019). Wenngleich die Pilotstudie zunächst auf die Validierung des Tests gerichtet war, lassen sich auch bereits erste empirische Befunde zum Kompetenzstand von Lehrenden berichten. So identifizieren die Autoren sechs Faktoren mit insgesamt 33 Items, die 55\% der Varianz erklären und den oben beschriebenen Facetten des medienpädagogischen Kompetenzmodells zugeordnet werden können. Dazu zählen (1) der Umgang mit medienbezogenen Bedürfnissen der Teilnehmenden, (2) medienbezogene Teilnehmerorientierung, (3) mediendidaktische Kompetenz, (4) Wissen über die medienbezogene Umwelt der Teilnehmenden, (5) Ablehnen digitaler Medien für den Lehreinsatz und schließlich (6) Kursvorbereitung mit digitalen Medien. Mittels einer hierarchischen Clusteranalyse konnten aus den sechs Faktoren fünf verschiedene Cluster gebildet werden: Knapp die Hälfte der Befragten (47\%) fielen in den Cluster mit durchschnittlichen medienpädagogischen Kompetenzen, gefolgt von knapp 23\% mit geringem Wissen über die medienbezogene Umwelt ihrer Adressaten. Etwa $6 \%$ wiesen eine geringe medienbezogene Teilnehmerorientierung auf, $10 \%$ verfügen über nur geringe mediendidaktische Kompetenz und rund $14 \%$ lehnten den Medieneinsatz in der Lehre ab (ebd., S. 318 f.). Lehrende mit durchschnittlichen

1 „DigCompEdu Check-In“ für Lehrende in der Erwachsenenbildung: http://europa.eu/!ut86vP 
Kompetenzen verwenden digitale Medien zur Kursvorbereitung und lehnen den Einsatz digitaler Medien in Kursen nicht ab. Im Cluster mit ablehnender Haltung versammelt sich eine signifikant höhere Zahl von Lehrenden aus den Bereichen Gesundheit und Sport. Trotz der hohen Fallzahl der Studie ist die Repräsentativität der Ergebnisse nicht gegeben, wie die Autoren betonen: Die Stichprobe beinhaltet vor allem Lehrende der Volkshochschulen, zudem sind weibliche Lehrende deutlich überrepräsentiert und die Erhebungsmethode mittels Online-Fragebogen dürfte insbesondere Teilnehmende mit passenden digitalen Nutzungsgewohnheiten angesprochen haben (Rott \& Schmidt-Hertha, 2019). Weitere auf Basis der Daten publizierte Erkenntnisse zeigen, dass altersbezogene Unterschiede in Bezug auf medienpädagogische Kompetenzen bei Lehrenden lediglich im Bereich Mediendidaktik bedeutsam sind (ebd.).

Der breit rezipierte Bertelsmann Monitor Digitale Bildung lässt mittelbar auf vorhandene Kompetenzen bei Lehrenden schließen. Er diagnostiziert anhand der quantitativen Auswertung einer nicht-repräsentativen Befragung von 260 Lehrenden, dass die Befragten zwar vielfältig digitale Werkzeuge nutzen, jedoch vor allem zur Vor- und Nachbereitung der eigenen Kurse bzw. zur Durchführung von Prüfungen. Dagegen verwenden sie nur eine sehr eingeschränkte Auswahl bei der methodischdidaktischen Gestaltung im Kurs selbst. Hier ist auffällig, dass es sich bei den häufig in den Kursen eingesetzten Medien vor allem um solche handelt, die eher auf Rezeption ausgelegt sind (etwa Powerpoint, Videos und digitale Texte) und diese meist ergänzend eingesetzt werden (Schmid, Goertz \& Behrens, 2018, S. 30 ff.). Umfassende empirische Bestandsaufnahmen der digitalen Kompetenzen von Lehrenden in der EB/WB finden sich im betrachteten Zeitraum ansonsten weder in deutschsprachigen noch in internationalen Publikationen.

Fünf Studien im ermittelten Korpus thematisieren veränderte bzw. neue Kompetenzanforderungen durch digitale Technologien. Die methodischen Zugänge der Arbeiten sind sehr unterschiedlich; es handelt sich ausschließlich um internationale Publikationen.

Ferguson et al. (2018) untersuchen in einem narrativen Review von 167 Publikationen des internationalen FutureLearn Academic Network (FLAN) den Stand der Forschung zu MOOCs. Sie stellen fest, dass digitale Technologien Lernszenarien ermöglichen, die in bisher nicht gekanntem Maße skalierbar sind und entsprechend hohe Ansprüche an die Organisation und Durchführung stellen, wenn sie effektiv sein sollen. Zur Bewältigung der vielfältigen Aufgaben müssten Teams entwickelt werden, die sich entsprechend den unterschiedlichen Anforderungen (wie zum Beispiel der Bearbeitung von Lizenzfragen, dem didaktischen Design oder zur technischen Umsetzung bzw. Unterstützung) zusammensetzen (Ferguson et al., 2018, 206f.).

Tan (2018) analysiert anhand eines Reviews von drei Fallstudien aus den Bereichen wissenschaftliche Weiterbildung, Hochschule und Lehrerfortbildung verschiedene Herangehensweisen an Technologieintegration im Zusammenhang mit 
lebenslangem Lernen, die jeweils verschiedene Aspekte von Lernen fokussieren (Wissenserwerb, Teilhabe, Wissensgenerierung) und erarbeitet Implikationen für Lehrende. Er betont die Notwendigkeit einer systematischen Herangehensweise an den Technologieeinsatz in der Lehre, die vom individuellen Verständnis von Lehren und Lernen ausgeht, statt von den Grenzen und Möglichkeiten der Technologie.

Paepe, Zhu und Depryck (2018) bearbeiten in einer qualitativen Interviewstudie mit 13 Sprachlehrenden bzw. Stakeholdern aus dem Bereich Sprachen die Frage, welche Vor- und Nachteile diese in Bezug auf Online-Sprachlernangebote wahrnehmen. Die Befragten nennen die soziale Interaktion mit Teilnehmenden in OnlineUmgebungen als eine besondere Herausforderung, ebenso wie das Herstellen eines lernförderlichen Gemeinschaftssinns innerhalb der Gruppe (ebd, S. 26, S. 28).

Loizzo et al. (2017, S. 17 f.) stellten in einer Mixed-Methods-Studie durch quantitativ-deskriptive Auswertung einer Befragung von 671 Teilnehmenden eines MOOCs sowie der inhaltsanalytischen Auswertung von 12 Videointerviews fest, dass Lehrende gefordert sind, verschiedene MOOC-Konzepte zu kombinieren, um der großen Zahl und Heterogenität der Lernenden adäquat gerecht werden zu können. Gleichzeitig müssen sie die Umsetzung dynamisch und flexibel handhaben, um dauerhafte Responsivität gegenüber den Lernenden und ihren Lebensumständen zu gewährleisten.

Laurian-Fitzgerald und Kollegen leiten auf Basis eines Reviews und eigener Erfahrungen aus der Umsetzung diverser Online-Studiengängen sowie einer Befragung von 241 Studierenden eine Reihe an Empfehlungen für den Umgang mit online lernenden Erwachsenen ab. Lehrende müssen demnach insbesondere dazu fähig sein, konstruktiv mit Diversität umzugehen, Kurse lernendenzentriert zu entwickeln und kompetente sowie andauernde Lernbegleitung $\mathrm{zu}$ bieten (LaurianFitzgerald et al., 2018, S. 155, S. 160 ff.).

\subsection{Implikationen der Digitalisierung für die Haltung und die Rolle von Lehrpersonal}

Es fanden sich im Korpus drei Studien mit Fragestellungen zur pädagogischen Haltung in Bezug auf die Digitalisierung in der EB/WB (Bolten \& Rott, 2018; Burchert \& Grobe, 2017; Colliander, 2019).

Die Haltung der Lehrenden gegenüber digitalen Medien ist nach Meinung der Akteure in der EB/WB ausschlaggebend für die Verwendung dieser Medien in LehrLern-Situationen. Dies stellten Bolten und Rott in ihrer qualitativen Studie zu medienpädagogischen Kompetenzen in Interviews und Fokusgruppen fest (Bolten \& Rott, 2018, S. 147).

In einer Studie zur Implementierung digital gestützter beruflicher Weiterbildung fanden Burchert und Grobe in Interviews mit 14 Lehrenden und Verantwortlichen im Bildungsmanagement neben einer grundsätzlichen Skepsis bei einigen Befragten 
heraus, dass die meisten Interviewten das selbstorganisierte Lernen in Verbindung mit dem Einsatz von digitalen Medien grundsätzlich als pädagogische Chance begreifen (Burchert \& Grobe, 2017, S. 8). Die befragten Lehrkräfte sehen digitale Weiterbildungsangebote in direktem Zusammenhang mit selbstorganisiertem Lernen und bestätigen somit die Verschiebung der Rolle vom Lehrenden zum Lernbegleitenden (Buchert \& Grobe, 2017). Die ,individuelle Haltung zum (selbstorganisierten/lebenslangen) Lernen“ identifizieren Burchert und Grobe als eine der zentralen Herausforderungen bei der Implementierung digital gestützter beruflicher Weiterbildung (ebd., S. 7).

Welche Haltung Lehrkräfte in der Erwachsenenbildung zu Veränderungen unter anderem durch die Digitalisierung einnehmen, untersuchte Colliander in einer Befragung von schwedischen Zweitsprachlehrenden für Erwachsene. Sie befragte 13 Personen in einer qualitativen Interviewstudie. Colliander konnte zeigen, dass das Selbstverständnis Lehrender durch äußere Einwirkungen und aktiv durch die Lehrenden selbst verändert wird. Globale Entwicklungen wiederum, etwa die Digitalisierung, verwandeln auch die Lehrpraxis. Die Befragten äußerten, dass digitalisierte Lehre den Unterricht weniger lehrerzentriert machten (Colliander, 2019, S. 64). Die Reaktion der Lehrenden darauf hängt zum einen damit zusammen, wie diese die Veränderungen in Bezug auf ihre eigene Lehre und die Lernenden bewerteten. Zum anderen ist sie von der individuellen Situation und den individuellen Erfahrungen der Lehrperson abhängig. Verbesserten die Veränderungen Lehre und Lernen, wurden sie angenommen. Veränderungen, die in den Augen der Lehrenden einen negativen Effekt auf Lehren und Lernen hatten, wurden abgelehnt (ebd., S. 68).

Zwei Studien betrachten Rollenveränderungen bei Lehrpersonal aus Lehrendenperspektive (Paepe et al., 2019; Roberts, 2018), eine verknüpft die Perspektiven von Lehrenden und Lernenden (Cendon, 2018). Zwei Studien untersuchen den Umgang mit digitalen Tools und schließen daraus auf die Lehrendenrolle (OER bei Littlejohn \& Hood, 2016; Social-Media-Tools bei Montebello, 2017).

Eine qualitative Interviewstudie von Paepe et al. mit 13 Lehrenden bzw. Anbietern von Online-Sprachlernkursen zeigte unter anderem, dass die Befragten als kritischen Erfolgsfaktor für Online-Angebote einen Mangel an Motivation und Offenheit bei Lehrkräften feststellten, verbunden unter anderem mit der Furcht vor einem Wandel ihrer Lehrpraxis (Paepe et al., 2019, S. 282).

Roberts untersuchte in einer quantitativen Querschnittsstudie 119 Datensätze aus einer Online-Umfrage zur Rollenwahrnehmung bei in der Fernlehre tätigen Lehrenden und Forschenden an der University of South Africa. Ziel war es, zur aktuellen und zukünftigen Rolle der Fernlehrenden Kompetenzen zu ermitteln und Informationen für die zukünftige Gestaltung von beruflicher Weiterbildung am Fernlehre-Zweig der Universität zu erhalten. Grundlage der Befragung war eine Liste von zehn Rollenbeschreibungen. Bei zwei davon zeigte sich, dass sich die Einschätzung seitens der Lehrenden zu deren aktueller und zukünftiger Bedeutung deutlich verschiebt. Wichtiger werde die Rolle des Technologie-Experten und des 
Instructional Designers, Bereiche, in denen die Befragten ihre eigenen Kompetenzen als eher gering einschätzten und für die sie sich Weiterbildung erhofften (Roberts, 2018, S. 51).

Ergebnisse aus zwei Befragungen verknüpft Cendon (2018) in ihrer Untersuchung mit dem Ziel, aus der Perspektive der wissenschaftlichen Weiterbildung ein Rollenkonzept der Lehrenden zu entwerfen. Sie wertete Interviews mit 26 Studierenden sowie die Motivationsschreiben und zwei Gruppendiskussionen (mittels qualitativer Inhaltsanalyse nach Mayring) und sieben Experteninterviews mit Lehrenden in Weiterbildungsstudiengängen (mittels Grounded Theory) aus. Cendon betont die Bedeutung der Verknüpfung von Lehrenden- und Lernenden-Perspektive bei der Untersuchung von Lehr-/Lernpraktiken. Dies gelte umso mehr, wenn neue Konzepte vernetzten Lernens durch digitale Technologien die Grenzen zwischen Lernenden und Lehrenden verwischten (Cendon, 2018, S. 82). Cendon stellt fest, dass Lernende die Vielfalt von Lehrendenrollen und -strategien eher positiv bewerten und sieht Unterstützungsbedarf bei den Lehrenden in pädagogischen und technischen Fragen. Sie verweist auf den Vorschlag von Willcox, Sarma und Lippel (2016), einen „learning engineer" innerhalb der Weiterbildung an Hochschulen einzusetzen (Cendon, 2018, S. 86). Dies wäre nach Willcox et al. (2016, S. 25 f.) eine Person, die Kompetenzen zu Lernen, Technologie und Lehren in sich vereint.

Littlejohn und Hood fanden in einer Studie zum Wissenserwerb von Erwachsenenbildnern am Arbeitsplatz Hinweise darauf, dass veränderte professionelle Praktiken auch die Rolle des Lehrenden und des Lerners verändern (Littlejohn \& Hood, 2016, S. 506). Sie betrachteten die Auswirkungen der Nutzung von OER (Open Educational Resources, dt. offene Bildungsmaterialien, die meist in digitaler Form vorliegen) auf Lehrende in Hinblick auf deren Expertise und Wissen. In einer Online-Umfrage befragten die Autoren 521 Lehrende. Der überwiegende Teil der Befragten arbeitete in Großbritannien im Bereich der Hochschullehre. Für die Auswertung nutzten sie ein leicht modifiziertes, bereits validiertes Instrument zur Messung von Lernhandlungen am Arbeitsplatz (WLA scale, vgl. Fontana, Milligan, Littlejohn \& Margaryan, 2015). Sie kommen zu dem Ergebnis, dass Lehrende durch den Umgang mit OER das nötige Wissen erwerben, um ihre Lehrpraxis weiterzuentwickeln (Littlejohn \& Hood, 2016, S. 506 f.).

Rückschlüsse auf das Rollenbild von Lehrenden in der EB/WB lässt Montebellos Online-Befragung (Montebello, 2017) von 80 Lehrenden in einem Kurs zur beruflichen Weiterbildung in acht europäischen Partnerorganisationen der Grundbildung zu. Er untersuchte mit Methoden der Grounded Theory deren Umgang mit 31 digitalen Tools, deren Beschreibungen er Blooms Lernziel-Taxonomie zuordnete. Kreativität, Freiheit und Flexibilität nannten die Befragten als Vorteile, Frustration und große Anstrengung beim Lernen als Nachteile beim Einsatz der digitalen Tools. Die Befragten sprachen sich für den Einsatz von digitalen Tools unter klaren pädagogischen Zielsetzungen aus. Montebello stellt fest, dass „digitale Pädagogik“ (im engl. Original digital pedagogy) notwendigerweise ein Bestandteil beruflicher 
Weiterbildung von Lehrenden sein muss, um den ständigen Veränderungen in dem Bereich gewachsen zu sein. Dennoch werde die „klassische Rolle des Lehrenden als Leitender, Begleitender und Führender" weiterhin für den effektiven Lernprozess unabdingbar bleiben (ebd., S. 165).

\subsection{Implikationen der Digitalisierung für die Aus- bzw. Fortbildung von Lehrpersonal}

Fünf Studien im Korpus machen Aussagen zum (digitalen) Fortbildungsverhalten von Lehrenden (Autorengruppe wb-personalmonitor, 2016; Mavropoulos, Sipitanou \& Pampouri, 2019; Bolten et al., 2019; Schmid, Goertz \& Behrens, 2018; Rohs \& Bolten, 2017). Acht Studien im Korpus tragen empirische Erkenntnisse zu verschiedenen Aspekten der (informellen) Fortbildung von Lehrenden bei: zu Effekten von Qualifikation (Klauser \& Schlicht, 2016), zum Zusammenhang von Vertrautheit und Nutzung digitaler Technologien (Pérez-Paredes, Ordoñana Guillamón \& Aguado Jiménez, 2018; Schmid, Goertz \& Behrens, 2018; Schrader \& Schöb, 2016), sowie zur Gestaltung erfolgreicher Fortbildungen (Nafukho, Alfred, Chakraborty, Johnson \& Cherrstrom, 2017; Schöb, Rohs, Biel \& Scholze, 2016). Eine Studie evaluiert die Eignung eines digitalen Lernformats für grundständige Ausbildung von Lehrenden in der EB/WB (Staab \& Egetenmeyer, 2019).

Lehrende profitieren allgemein von Fort- und Weiterbildungsmaßnahmen (vgl. Marx, Goeze, Kelava \& Schrader, 2018). Der eingangs skizzierte Fortbildungsbedarf trifft auf eine - verglichen mit der Fortbildungsbereitschaft Erwachsener allgemein überdurchschnittliche Fortbildungsbereitschaft der Lehrenden. Dies gilt selbst dann, wenn den Lehrenden dadurch Kosten entstehen (Autorengruppe wb-personalmonitor, 2016, S. 125f.). Diese Beobachtung bestätigt sich auch für digitale Formate und andere nationale Bildungsräume. Entsprechendes haben z.B. Mavropoulos und Kollegen für Lehrende in der Erwachsenenbildung in Griechenland mittels einer summativen Evaluationsstudie eines Blended-Learning-Programms nachgewiesen (Mavropoulus et al., 2019, S. 283).

Allerdings deutet sich eine Diskrepanz zwischen dem allgemeinen Fortbildungsverhalten von Lehrenden und der Teilnahme an Maßnahmen zum Erwerb digitaler Kompetenzen an: Während von den 1338 befragten Lehrenden des wb-Personalmonitors je nach Beschäftigungsform zwischen 64 (hauptberufliche Honorarkräfte) und über $80 \%$ (Selbstständige bzw. Angestellte in der Weiterbildung) in den letzten 12 Monaten an einer Fortbildung teilgenommen haben (Autorengruppe wbpersonalmonitor, 2016, S. 125f.), stellen Bolten et al. (2019, S. 316) in Bezug auf Fortbildungen zu digitalen Kompetenzen fest, dass $45 \%$ der 622 befragten Lehrenden an keiner derartigen Veranstaltung im Verlauf der letzten fünf Jahre teilgenommen haben. Ein Drittel gab an, in diesem Zeitraum eine bis zwei Fortbildungsveranstaltungen mit Bezug zu digitalen Medien besucht zu haben, $20 \%$ 
besuchten mehr als drei Veranstaltungen. Berücksichtigt man diese Differenz in den Quoten in Verbindung mit den unterschiedlichen Bezugszeiträumen der beiden Studien von 12 Monaten gegenüber fünf Jahren so lässt sich begründet vermuten, dass Lehrende Fortbildungen zu digitalen Themen eher seltener als andere besuchen. Dazu passt die Feststellung aus dem Bertelsmann Monitor, dass sich die Lehrenden die notwendigen Kompetenzen vor allem mittels Selbststudium bzw. informellem Lernen aneignen (Schmid, Goertz \& Behrens, 2018, S. 36f.). In einer qualitativen Studie zum Verlauf der medienpädagogischen Professionalisierung wurden Lehrende an Volkshochschulen in themenzentrierten Interviews befragt (Rohs \& Bolten, 2017). Rohs und Bolten arbeiten heraus, dass die Aneignung medienpädagogischer Kompetenzen bzw. Wissens sehr heterogen verläuft. Sie vollzieht sich in einem Wechselspiel von informellem, non-formalem und formalem Lernen, wobei ersteres für alle untersuchten Lehrenden aus zeitökonomischen Gründen besonders bedeutend ist. Zugleich benennt die Studie intrinsische Motivation als bedeutenden Faktor für die Entwicklung von medienpädagogischer Kompetenz (ebd., S. 38 f.).

Im Rahmen einer Untersuchung der Wirksamkeit und Wirtschaftlichkeit eines technologiegestützten Fernlehrgangs im Vergleich zu einem Präsenzlehrgang befragten Klauser und Schlicht zwölf Lehrpersonen. Sie kommen zu dem Schluss, dass von den untersuchten Lehrpersonen solche mit teletutorieller Zusatzausbildung den Kolleginnen und Kollegen ohne eine solche Qualifikation in einer technologiegestützten Umgebung in didaktisch-methodischer Hinsicht überlegen sind. Dies betrifft zum Beispiel das adäquate Formulieren von Aufgaben, das Kommunizieren von Lernzielen oder Feedback (Klauser \& Schlicht, 2016, S. 182).

Peréz-Paredes et al. untersuchten in einer quantitativen Studie mit spanischen und britischen Sprachlehrkräften die Kenntnis und Nutzung von mobilen bzw. offenen Technologien. Sie zeigten, dass die Vertrautheit mit digitalen Tools mit dem Grad der Qualifikation der Lehrkraft verknüpft ist und signifikant mit der tatsächlichen Nutzung digitaler Werkzeuge durch Lehrende korreliert, jedoch nicht mit dem Alter der Lehrkraft oder der Berufserfahrung (Pérez-Paredes et al., 2018, S. 531 f.). Den Zusammenhang zwischen der Vertrautheit mit und der Nutzung von digitalen Technologien durch Lehrende deutet auch der Bertelsmann Monitor Digitalisierung für das deutsche Weiterbildungspersonal an: Die Zahl der Lehrenden, die zum Zeitpunkt der Erhebung in den letzten 12 Monaten eine OnlineVeranstaltung durchgeführt hatten, liegt hier bei $27 \%$, für gemischte Formate und für Blended Learning bei $23 \%$. Im Vergleich antworteten $22 \%$ der Teilnehmenden, dass sie häufig E-Learning-Veranstaltungen durchführen und $19 \%$ gaben an, häufig Blended-Learning-Konzepte zu verwenden (Schmid, Goertz \& Behrens, 2018, S. 32). Kenntnis und Nutzung von digitalen Werkzeugen kann zur Verbesserung der Kursplanung beitragen, wie Schrader und Schöb in einer quasi-experimentellen Interventionsstudie am Beispiel einer Kursplanungs-App mit angehenden Lehrkräften der Erwachsenenbildung zeigen (Schrader \& Schöb, 2016). 
Zur Frage, welche Faktoren für die erfolgreiche (digitale) Fortbildung von Lehrenden in der EB/WB bedeutend sind und wie sie gestaltet sein sollten, findet sich in der berücksichtigten Literatur eine quasi-experimentelle Querschnittsstudie mit 251 Lehrenden der Erwachsenenbildung im Rahmen einer Blended-LearningIntervention. Untersucht wurde, inwiefern sich die Eigenschaften der Lernenden, die Gestaltung der Fortbildung sowie die Arbeitsbedingungen in der Praxis auf den Transfer des Gelernten in die eigene Praxis auswirken. Die Autoren identifizieren drei signifikante Faktoren für erfolgreichen Lerntransfer: die intrinsische Lernmotivation der Lernenden, die Anforderung und Unterstützung der Arbeitsstelle und schließlich die effektive und praxisrelevante Gestaltung der Fortbildung (Nafukho et al., 2017, S. 347 ff.). Ein Ansatz für die erfolgreiche Konzeption von Online-Lernformaten für Lehrende der Erwachsenenbildung kann dabei die enge Abstimmung mit den Lehrenden sein, wie Schöb et al. in ihrer multimethodischen Bedarfserhebung feststellen (Schöb et al., 2016). Die Ergebnisse dokumentieren eine hohe Akzeptanz der Lehrenden gegenüber Inhalten, die mittels Fokusgruppen eng mit den Anforderungen der Praxis verknüpft wurden. Zudem zeigen sie, dass die Mitwirkungsbereitschaft an Online-Fortbildungsformaten von der Medienaffinität und dem Ergebnis einer kritischen Kosten-Nutzen-Abschätzung durch die Lehrenden beeinflusst wird.

Zur Integration digitaler Technologien in die grundständige Ausbildung von Lehrenden findet sich im ermittelten Korpus eine Studie von Staab und Egetenmeyer. Sie evaluierten ein Blended-Learning-Arrangement im Rahmen des Erasmus+Projekts COMPALL. Im Rahmen des Projekts wurde ein Studienmodul zum Thema „international vergleichenden Forschung in der Erwachsenenbildung und des lebenslangen Lernens" entwickelt und als Blended-Learning-Format umgesetzt (Staab \& Egentenmeyer, 2019, S. 282). Das Modul wurde mit Hilfe einer quantitativ ausgewerteten Befragung evaluiert, an der Studierende eines Masterstudiengangs Erwachsenenbildung bzw. Promovierende in diesem Bereich teilnahmen. Die Autorinnen stellten fest, dass das Format für die Ausbildung von Lehrenden der EB/WB geeignet ist. Es ermögliche nachhaltige Lernprozesse sowie das Aufgreifen verschiedener Lernbedarfe auf Seiten der angehenden Lehrenden und leiste einen Beitrag zur Entwicklung kognitiver, motivationaler und sozial-kommunikativer Kompetenzen (ebd., S. 288).

\subsection{Zukünftige Entwicklungen im Umfeld der Lehrenden durch die Digitalisierung in der Erwachsenenbildung}

Bei den betrachteten Veröffentlichungen gibt es zwei Berichtsreihen, die explizit Fragen zu zukünftigen Entwicklungen erforschen, die die Digitalisierung im Bildungsbereich betreffen. 
Auf der Grundlage eines modifizierten Delphi-Prozesses analysiert ein international besetzter Beirat aus Expertinnen und Experten seit 2002 globale Entwicklungen im sogenannten Horizon Project. Jedes Jahr werden im Horizon Report sechs Trends, sechs Entwicklungen und sechs Herausforderungen in Bezug zu Bildungstechnologien und -praktiken beschrieben, die wahrscheinlich in den kommenden fünf Jahren bestimmend in diesem Sektor werden könnten. Die Untersuchungsergebnisse sollen die Technologieplanung von Lehrenden, (Hochschul-) Leitungen, Entscheidern in der Politik und Technologie-Experten unterstützen (Alexander et al., 2019, S. 3).

Die Ergebnisse für den Bereich Hochschulbildung sind für den Sektor der Erwachsenen- und Weiterbildung interessant, da Überschneidungen zur Hochschulbildung im Bereich der wissenschaftlichen Weiterbildung oder der Angebote von sogenannten „offenen“ Hochschulen (z. B. MOOCs) gegeben sind. Der Horizon Report nennt Trends und Herausforderungen für drei Zeitfenster (kurzfristig: in ein bis zwei Jahren, mittelfristig: in drei bis fünf Jahren, langfristig: in fünf oder mehr Jahren) und teilt die identifizierten Herausforderungen in drei abgestufte Kategorien ein: lösbar, schwierig und komplex. Im betrachteten Zeitraum lässt sich anhand der Prognosen des Horizon Projects im Zeitverlauf vor allem der Trend des Neudenkens der Lehrendenrolle nachverfolgen: Der Report von 2017 bezeichnet diese Herausforderung in seiner Hochschulausgabe (engl. Originaltitel: Higher Education) erstmals als eine der komplexen anstehenden Herausforderungen beim Einsatz moderner Technologie in der Bildung für die kommenden fünf Jahre (Adams Becker et al., 2017, S. 34 f.). Damit platziert der Report das Thema auf der höchsten Stufe, vorbehalten für Herausforderungen, deren Definition und Handhabung sehr anspruchsvoll sind. Die Experten gehen davon aus, dass von Lehrenden zunehmend erwartet wird, dass sie eine Auswahl an technologiebasierten Tools nutzen und sich in Online-Diskussionen oder durch kollaborative Mitwirkung einbringen. Die durch den Einsatz von digitaler Technologie geförderte Zunahme kompetenzorientierter Lehr-Lern-Arrangements verstärkte diese Herausforderung. (Adams Becker et al., 2017, S. 34). Der Horizon Report 2018 übernahm diese Einschätzung. Die Herausforderung wurde immer noch als sehr anspruchsvoll eingestuft (Adams Becker et al., 2018, S. 34f.). Der Horizon Report 2019 nennt das Neudenken der Lehrpraxis in der gleichen Kategorie und stellt dies in Zusammenhang mit der Rollenverschiebung bei den Lehrenden. Diese habe den Bedarf einer Neubewertung verstärkt. Die Umgestaltung von Lehrangeboten aufgrund der Nutzung von digitalen Tools ermögliche es Lehrenden lernerzentrierte Ansätze zu nutzen, in denen sie als Lernbegleitende agieren. Berufliche Weiterbildung, die den Gebrauch digitaler Tools unterstütze, führe zur Zusammenarbeit in Instructional-Design-Teams und mit weiteren im Bildungsbereich Tätigen, und beschleunige die Anwendung neuer Lehrpraxen (Alexander et al., 2019, S. 19). Die Rolle der Lehrenden und ihre Verwandlung durch digitale Ressourcen, Internet und mobile Geräte war bereits 2013 im Horizon Report als ein Schlüsseltrend genannt worden. Lehrende würden 
sich in Zukunft eher als Guides bei der Auswahl von Lerncontent verstehen, hieß es dort (Johnson et al., 2013, S. 8).

Eine weitere zweistufige Delphi-Studie erstellte das $\mathrm{mmb}$ Institut, um wichtige Elemente des digitalen Lernens $\mathrm{zu}$ untersuchen. Die Studie betrachtet die Bildungssektoren Schule, Ausbildung, Hochschule und Weiterbildung für den Zeithorizont bis 2025. 68 Expertinnen und Experten aus Deutschland, Österreich und der Schweiz arbeiteten daran mit und legten sieben Thesen vor. Relevant im Zusammenhang mit diesem Review ist insbesondere die These, dass die größte Herausforderung bei der Digitalisierung der Bildung allgemein und damit auch im Bereich der Weiterbildung die digitale Kompetenz der Lehrenden darstellt (4,4 von 5 auf einer 5-stelligen Skala, 5 entspricht sehr großer Herausforderung; mmb Institut, 2016, S. 3). Danach rangieren Didaktische Ansätze (4,2 für alle Bildungssektoren) und als drittes im Bereich Weiterbildung die Digitale Kompetenz der Entscheider (4,0 für alle Bildungssektoren). Eine zweite Befragungswelle (Delphi-Stufe 2) fragte die Experten nach den ihrer Meinung nach wichtigsten Maßnahmen für den jeweiligen Bildungssektor. Für den Bereich Weiterbildung nannten die Befragten neben Weiterbildung, Qualifizierung, Anreizmodelle, Ausstattung der Einrichtungen und Qualitätssiegel für gute Angebote auch das Vorantreiben des Rollenwandels der Lehrenden vom Dozenten zum Coach (mmb Institut, 2016, S. 6-7).

Das mmb Institut veröffentlicht jährlich zudem die Trendstudie mmb Learning Delphi. Jedes Jahr werden in einem zweistufigen Delphi-Prozess E-LearningExpertinnen und -Experten aus Deutschland, Österreich und der Schweiz zu Trends im digitalen Lernen befragt. Im Zentrum stehen Themen mit wirtschaftlichem Bezug, da es um Entwicklungen im Bereich der Weiterbildungswirtschaft geht. Verfolgen lassen sich Aussagen der Expertinnen und Experten zur Bedeutung von Anwendungen als Lernform in Unternehmen (mmb Trendmonitor I/2017, S. 5-6, mmb Trendmonitor 2017/2018, S. 5-6, mmb Trendmonitor 2018/2019, S. 6-7) jeweils anteilig nach ihrer Zustimmung zur Frage „Werden die folgenden Anwendungen in den kommenden drei Jahren eine zentrale Bedeutung oder eine geringe Bedeutung als Lernform für das betriebliche Lernen in Unternehmen haben?“. Die Befragten nannten in den Untersuchungen seit 2016 Blended Learning (2018 $97 \%$ ), Videos (2018 $94 \%$ ), Micro Learning/Learning Nuggets (2018 91\%) und mobile Anwendungen/Apps (2018 89\%) als die Anwendungen, die ihrer Ansicht nach in den kommenden drei Jahren eine zentrale Bedeutung als Lernform haben werden (mmb Trendmonitor I/2017, S. 5; mmb Trendmonitor 2017/2018, S. 5; mmb Trendmonitor 2018/2019, S. 6). 


\section{Zusammenfassung und Diskussion}

Insgesamt besteht in der Forschung auf theoretischer und konzeptioneller Ebene Konsens darüber, dass die Digitalisierung bestehende Kompetenzanforderungen verändert bzw. neue Anforderungen schafft (Pachner, 2018; Rohs, Bolten \& Kohl, 2017; Sgier et al., 2018). Das ermittelte Korpus beinhaltet drei Kompetenzmodelle, welche die Anforderungen an Lehrende durch die Digitalisierung systematisch zu erfassen suchen: das allgemeine Kompetenzmodell GRETA für Lehrende mit Aspekten digitaler Kompetenzen (Strauch et al., 2019), das auf EU-Ebene entwickelte, bildungsbereichsübergreifende Rahmenmodell für digitale Kompetenzen von Lehrenden DigCompEdu (Redecker \& Punie, 2017), sowie das medienpädagogische Kompetenzmodell (Rohs, Rott et al., 2017). Jedes der Modelle ist theoretisch fundiert und wurde im Entstehungsprozess durch Expertinnen und Experten und/ oder Akteurinnen und Akteure aus der Praxis validiert. Eine weitere Gemeinsamkeit ist die Differenzierung der Anforderungen auf verschiedenen Abstraktionsebenen. Bisher wurde lediglich das medienpädagogische Kompetenzmodell für den Bereich EB/WB in einer Pilotstudie empirisch validiert, wobei die Ergebnisse nicht repräsentativ sind und zum Zeitpunkt der Fertigstellung dieses Reviews nur in Teilen publiziert sind (Bolten et al., 2019; Rott \& Schmidt-Hertha, 2019). In den empirischen Studien, die sich mit den konkreten Veränderungen der Anforderungen an Lehrende befassen, werden diese als systematische Konzeption, Planung und Durchführung (Ferguson et al., 2018; Tan, 2018), Teamwork (Ferguson et al., 2018), Kommunikation (Kennedy et al., 2016; Paepe et al., 2018), Flexibilität, (Loizzo et al., 2017) Lernendenorientierung und -begleitung (ebd.; Laurian-Fitzgerald et al., 2018), Umgang mit Diversität/Heterogenität (ebd.), Medienkompetenz und Motivation sowie Umgang mit Unsicherheit seitens der Lehrenden (Kennedy et al., 2016) beschrieben. Diese Kompetenzanforderungen lassen sich im GRETA-Kompetenzmodell verorten. So lässt sich feststellen, dass sich die Digitalisierung vor allem auf das berufspraktische Wissen und Können auswirkt. Dabei sind die im Modell benannten Bereiche Didaktik und Methodik (Loizzo et al., 2017), Kommunikation und Interaktion (Kennedy et al., 2016; Laurian-Fitzgerald et al., 2018; Paepe et al., 2019), Beratung (Laurian-Fitzgerald et al., 2018) sowie Organisation (Ferguson et al., 2018, Loizzo et al., 2017) besonders betroffen. Auch Facetten der professionellen Selbststeuerung in GRETA, beispielsweise der Umgang mit Feedback oder berufliche Weiterentwicklung werden berührt (Kennedy et al., 2016).

$\mathrm{Zu}$ den tatsächlich vorhandenen digitalen Kompetenzen ist die empirische Erkenntnislage im Korpus mit zwei nicht-repräsentativen Studien sehr eingeschränkt: Demnach verfügen laut einer Clusteranalyse an die 50\% der Lehrenden lediglich über durchschnittliche digitale Kompetenzen. Die weiteren Gruppen sind eher als defizitär charakterisiert, was den Umgang mit digitalen Lernmedien betrifft, beispielsweise durch fehlende mediendidaktische Kompetenz oder geringem Wissen zur medienbezogenen Umwelt ihrer Adressaten (Bolten et al., 2019). 
Altersbezogene Unterschiede zeigen sich lediglich im Bereich Mediendidaktik (Rott \& Schmidt-Hertha, 2019). Lehrende nutzen digitale Medien vor allem zur Vor- und Nachbereitung und für Prüfungen. In der Kursgestaltung setzen sie nur eine eingeschränkte Auswahl überwiegend rezeptionsorientierter digitaler Medien ein (Schmid, Goertz \& Behrens, 2018).

$\mathrm{Zu}$ Kompetenzen fehlen repräsentative Bestandsstudien ebenso wie längsschnittliche qualitative und quantitative Studien, mit deren Hilfe sich Entwicklungen von Anforderungen bzw. die Kompetenzentwicklung bei Lehrenden nachzeichnen ließen. Die oben beschriebenen Modelle sind als Basis für solche Studien geeignet. Das GRETA-Modell (Strauch et al., 2019) zu allgemeinen Lehrkompetenzen hat den Vorteil, dass sich hier wie eben ausgeführt auch digitale Kompetenzen integrieren lassen und so beispielsweise auch Zusammenhänge von digitalen und nichtdigitalen Kompetenzen getestet werden können. Die Tatsache, dass sich die oben beschriebenen Kompetenzanforderungen auf das GRETA-Modell abbilden lassen, spricht aus Sicht der Verfassenden eher für eine Veränderung von bestehenden Kompetenzanforderungen an Lehrende durch die Digitalisierung und weniger für die Genese neuer Anforderungen. Im Sinne der Herstellung von internationaler, mindestens aber innereuropäischer Vergleichbarkeit bei der Kompetenzerfassung wäre qua Herkunft die Verwendung des DigCompEdu-Modells naheliegend. Dieses Modell müsste in entsprechenden Studien jedoch erst für den Bereich Erwachsenenbildung operationalisiert und empirisch validiert werden. Prinzipiell scheint jedes der hier vorgestellten Modelle geeignet, um als Basis für umfassende Bestandsaufnahmen der digitalen Kompetenzen von Lehrenden $\mathrm{zu}$ dienen. Die dadurch gewonnenen Informationen können wiederum Grundlage für Akteure und Akteurinnen in der EB/WB und der Bildungspolitik sein, um auf Basis wissenschaftlicher Evidenz wirkungsvolle Strategien zu entwickeln und sinnvolle Entscheidungen über Steuerungsmaßnahmen auf verschiedenen Ebenen zu treffen.

Die theoriebildende Forschung erkennt die professionelle Haltung von Lehrenden im Umgang mit digitalen Technologien und den insgesamt $\mathrm{zu}$ beobachtenden Rollenwandel vom Wissensvermittelnden hin zum Lernbegleitenden als zentrale Elemente bei der Digitalisierung der EB/WB (Bolten, 2018; Wienberger et al., 2018; Mayrberger, 2017). Die empirischen Arbeiten im Korpus stützen bzw. differenzieren diese Position: Für die Verwendung von digitalen Medien in Lehr-Lern-Situationen ist die Haltung der Lehrenden ausschlaggebend (Bolten \& Rott, 2018). Ein Mangel an Motivation und Offenheit bei Lehrkräften und deren Furcht vor einem Wandel ihrer Lehrpraxis sind kritische Faktoren bei der erfolgreichen Umsetzung von Online-Angeboten (Paepe et al., 2019). Beim Einsatz von digital gestützter beruflicher Bildung ist die individuelle Haltung von Lehrenden zum selbstorganisierten Lernen maßgeblich (Burchert \& Grobe, 2017). Ihre Haltung zu Veränderungen durch die Digitalisierung machen Lehrende abhängig von positiv bzw. negativ erlebten Effekten auf Lehren und Lernen (Colliander, 2019). Lehrende schätzen, dass ihre Rolle sich zukünftig weiter zum Technologie-Experten und Instructional 
Designer hin verschiebt (Roberts, 2018). Neue Konzepte vernetzten Lernens durch digitale Technologien verwischen die Rollengrenzen zwischen Lehrenden und Lernenden (Cendon, 2018). Die Nutzung von OER beim Wissenserwerb von Erwachsenenbildnern am Arbeitsplatz verändert die Rolle der Lehrenden hin zu mehr Partizipation der Lernenden (Littlejohn \& Hood, 2016). Lehrende setzen digitale Tools unter klaren pädagogischen Zielsetzungen ein und behalten dabei ihre klassische Rolle als Leitende (Montebello, 2017). Der Autor der letztgenannten Studie ist der einzige im betrachteten Konvolut, der aufgrund der Aussagen der befragten Lehrenden aus dem Bereich Grundbildung zu dem Ergebnis kommt, dass die Rolle des Lehrenden sich wenig verändern werde. Möglicherweise urteilen die Befragten so aufgrund der Lernenden-Zielgruppe, die besondere Ansprüche an die Anleitung in Lehr-Lernsituationen stellt. Andererseits bestätigt Montebello indirekt die Einschätzungen der oben zitierten Studien zu Herausforderungen bei der Digitalisierung der Bildung, die für die kommenden Jahre den Wandel der Lehrendenrolle als hochkomplexe Aufgabe formulieren (Alexander et al., 2019; Adams Becker et al., 2018; Adams Becker et al., 2017; mmb Institut, 2016; Montobello, 2017).

Die vorgestellten Publikationen zur Haltung bzw. Rolle von Lehrenden beschränken sich teilweise auf einzelne Kurse und Anwendungen (Paepe et al., 2019; Burchert \& Grobe, 2017; Montebello, 2017) oder institutionell begrenzte Untersuchungsgebiete (Roberts, 2018; Littlejohn \& Hood, 2016). Anhand umfassenderer Daten könnte indes Aus- und Weiterbildung geplant werden, denn Kompetenzentwicklung ist ein wichtiger Faktor in Hinblick auf einen Wandel von Haltung und Rolle (Colliander, 2019, S. 69). Das oben genannte GRETA-Modell (Bosche et al., 2018) bietet Kompetenzdefinitionen zur Professionellen Selbststeuerung, die Ansatzpunkte für Untersuchungen sein können. Längsschnittstudien oder idealerweise Panelstudien, die untersuchen, wie sich Haltung und Rolle im Laufe der Digitalisierung von Bildungsformaten entwickeln, könnten sich anschließen. Diese Methoden eignen sich insbesondere für die Untersuchung von Einstellungen und Haltungen, da Veränderungen über längere Zeiträume stattfinden (vgl. Mochmann, 2019).

Digitale Lehr- und Lernformate sind in der Praxis keine Seltenheit mehr (Schmid, Goertz \& Behrens, 2018). Das identifizierte Kompetenzdefizit (Mayert, 2018; Rohs, Rott et al., 2017) macht Fort- und Weiterbildungen von Lehrenden zwingend notwendig, so der Konsens in Theorie und Praxis (Mayert, 2018; Richter, 2017; Roberts, 2018; Rohs \& Weber, 2018; Sgier et al., 2018). Empirisch ist zunächst eine große Fortbildungsbereitschaft der Lehrenden festzustellen (Autorengruppe wb-personalmonitor, 2016), gleichwohl verläuft der Aufbau entsprechender Kompetenzen sehr heterogen, wobei intrinsische Motivation und individuelle Medienaffinität eine bedeutenden Rolle spielen (Nafukho et al., 2017; Rohs \& Bolten, 2017; Schöb, Rohs, Biel \& Scholze, 2016). Lehrende bilden sich eher informell bzw. durch Selbststudium fort und besuchen Veranstaltungen $\mathrm{zu}$ digitalen Themen seltener als andere tätigkeitsbezogene Weiterbildungsangebote (vgl. Bolten et al., 2019; Schmid \& Behrens, 
2017). Dabei profitieren Lehrende von der Teilnahme an Fortbildungsmaßnahmen: Sie agieren in technologiegestützten Umgebungen versierter, sind vertrauter mit digitalen Werkzeugen und setzen sie entsprechend häufiger ein (Klauser \& Schlicht, 2016; Pérez-Paredes et al., 2018; Schrader \& Schöb, 2016). Erfolgreiche Fortbildungen müssen effizient gestaltet sein und sich eng an den Anforderungen der Praxis orientieren (Schöb et al., 2016). Die Unterstützung von Kollegen und Kolleginnen oder der Bildungseinrichtung können zum Erfolg beitragen (Nafukho et al., 2017). Auch der Kompetenzaufbau im Rahmen der grundständigen Ausbildung von Lehrenden in der EB/WB kann vom Einsatz von Blended-Learning-Formaten profitieren (Staab \& Egetenmeyer, 2019).

Ähnlich wie in den anderen Teilfragestellungen fehlen in Bezug auf die Ausund Fortbildung grundlegende quantitative Daten, zum Beispiel zur Zahl und zum Inhalt von Fortbildungen zu digitalen Themen oder zur Bedarfslage seitens der Lehrenden. In Anbetracht der absehbar konstant hohen Entwicklungsdynamik digitaler Technologien und ihrem breiten gesellschaftlichen Einfluss gerät eine solche Bedarfserhebung zu einer Daueraufgabe, wie sie bereits für die empirische Erfassung digitaler Kompetenzen der breiten Bevölkerung Standard ist. Die große Bedeutung von informellen und non-formalen Lernprozessen in der Fortbildung von Lehrenden kann wahrscheinlich auf die Beschäftigungsstrukturen von Lehrenden in der EB zurückgeführt werden und deutet damit ein strukturelles Defizit mit Blick auf die Förderung von bzw. Möglichkeiten zum formalen Erwerb dieser Kompetenzen durch Lehrende an (Autorengruppe wb-personalmonitor, 2016, S. $125 \mathrm{ff}$.). Die Eignung von (teil-)digitalen Formaten für die Aus- und Fortbildung von Lehrenden allgemein wird in den ermittelten Studien explizit bekräftigt (Mavropoulos et al., 2019; Staab \& Egetenmeyer, 2019) oder nicht in Frage gestellt (Nafukho et al., 2017; Schöb et al., 2016). Die ermittelten Studien zu Effekten bzw. Erfolgsfaktoren von (teil-/digitalen) Fortbildungen für Lehrende sind allerdings ausnahmslos Evaluations- bzw. Fallstudien, die in ihren Bedingungen vielfältig und wenig kontrolliert sind, geschweige denn an etablierten Standards bzw. Modellen ausgerichtet. Dabei wird die Doppelrolle digitaler Technologien als Lerngegenstand und Lernmedium nicht reflektiert, ebenso wenig wie der Mangel an digitalen Kompetenzen in seiner Bedeutung als Zugangshürde zu digitalen Fortbildungsangeboten. Es finden sich keine Studien zur Frage, welche (teil-/digitalen) Formate in der Aus- und Fortbildung von Lehrenden wie stark geeignet sind, um spezifische digitale bzw. medienpädagogische Kompetenzen zu vermitteln. Es fehlen (vergleichende) quasi-experimentelle Studien zum digitalen Kompetenzaufbau von Lehrenden, die Technologien und Gestaltungsparameter systematisch variieren. Erkenntnisse zu Faktoren einer effektiven und nachhaltig erfolgreichen Implementation von Angeboten zum Erwerb digitaler Kompetenzen in der Aus- und Fortbildung von Lehrenden kommen im Bezugszeitraum nicht vor (Rohs \& Bolten, 2017, 310 ff.).

Mögliche Auswirkungen digitaler Technologien auf Lehrkräfte behandelt vor allem die theoriebildende Literatur (Bolten et al., 2018; Umbach et al., 2018). 
Hierin werden auch Handlungsaufforderungen an Praxis oder Politik formuliert (Kühn et al., 2018; Mayert, 2018; Rossmann, 2016), Bewertungen zum Stand der Digitalisierung vorgenommen (Arnold et al., 2017; Bremer, 2017) oder einzelne Lernanwendungen beurteilt (Niedermeier \& Müller, 2016; Staudacher, 2019; Wiemeyer, 2016). Prognosen auf empirischer Basis liegen im ermittelten Korpus ausschließlich als Studien vor, die nach der Delphi-Methodik vorgehen. Der Horizon Report nennt in den Ausgaben von 2017 bis 2019 das Neudenken der Lehrendenrolle bzw. das Neudenken der Lehrpraxis als hochkomplexe Herausforderungen für die nächsten fünf Jahre (Alexander et al., 2019; Adams Becker et al., 2018; Adams Becker et al., 2017). Die digitale Kompetenz der Lehrenden stellt eine sehr große Herausforderung bei der Digitalisierung der Bildung im Bereich der Weiterbildung dar. Wichtigste Maßnahmen im Bereich sind Fortbildung, Qualifizierung und auch das Anstoßen des Rollenwandels der Lehrenden (mmb Institut, 2016). Blended Learning, Videos, Micro Learning/Learning Nuggets und mobile Anwendungen/ Apps sind Anwendungen im Bereich E-Learning, die in den kommenden drei Jahren die meiste Bedeutung haben werden (mmb Trendmonitor I/2017; mmb Trendmonitor 2017/2018; mmb Trendmonitor 2018/2019).

Die von den betrachteten Delphi-Studien genannten Trends, Entwicklungen und Herausforderungen bieten Forschenden zahlreiche Anhaltspunkte für Untersuchungen etwa auf Ebene von Institutionen, Projekten und Praktikerinnen und Praktikern. Aus wissenschaftlicher Sicht ist dabei besonders die Frage relevant, ob sich die Konstanten aus den Trendberichten auch empirisch bestätigen lassen. Die Themen „Neudenken der Lehrendenrolle“ bzw. „Neudenken der Lehrpraxis“, die in den vergangenen drei Jahren als anspruchsvolle Herausforderungen für die nächsten fünf Jahre genannt wurden, böten sich hier an (Adams Becker et al., 2017; Adams Becker et al., 2018; Alexander et al., 2019). Die mmb-Trendstudien bieten jährlich ein Ranking einzelner Anwendungen als Lernformen in Unternehmen, so dass erfolgreiche „Aufsteiger“ identifiziert werden können, die konkrete Anlässe für Untersuchungen bieten.

\subsection{Implikationen und Desiderata für künftige Forschungsaktivitäten zum Thema}

Für alle betrachteten Aspekte der Gesamtfragestellung gilt, dass die empirische Erkenntnislage im betrachteten Zeitraum wenig gesättigt ist. Viele Studien dienen der Evaluation einzelner Interventionen oder Projekte und sind in Bezug auf die Fragestellung des Reviews nur für einzelne Aspekte ergiebig, z. B. die dargestellten Veränderungen von Kompetenzanforderungen (Paepe et al., 2018; Loizzo et al., 2017; Laurian-Fitzgerald et al., 2018), der Haltung bzw. Lehrendenrolle (Colliander, 2019; Paepe et al., 2019; Burchert \& Grobe, 2017; Montebello, 2017) oder im Bereich Fortbildung (Klauser und Schlicht, 2016; Pérez-Paredes et al., 2018; Nafukho et al., 
2017). Im Design dominieren Querschnittsstudien das Bild, Längsschnittstudien sind nicht vorhanden. Dabei überwiegen im Korpus qualitative gegenüber quantitativen Studien leicht (20 vs 14), Mixed-Methods-Designs und (quasi-)experimentelle Interventionsstudien sind mit gerade einmal drei bzw. einer Studie deutlich in der Minderheit (s. Tabelle 2). Großangelegte quantitative Studien sind rar: Sechs Studien können ein $n>300$ vorweisen, nur eine weist ein $n>700$ aus (s. Tabelle 2). Dieses Muster zeigt sich für die Erwachsenenbildung auch in internationalen Reviews (vgl. Boeren, 2017). Der wb-personalmonitor ist als einzige Studie im Korpus in Bezug auf Lehrende repräsentativ (Autorengruppe wb-personalmonitor, 2016, S. 31). Der breit rezipierte Bertelsmann Monitor Digitale Bildung kann ausschließlich für die befragten Bildungsadressaten Repräsentativität beanspruchen, nicht jedoch für die Lehrenden: Hier wurden lediglich 260 Personen befragt (vgl. Schmid, Goertz \& Behrens, 2018, S. 10). Insgesamt fehlen grundlegende quantitative Bestandserhebungen, beispielsweise hinsichtlich vorhandener Kompetenzen von Lehrenden oder des Fortbildungsangebots bzw. -bedarfs. Gerade mit Blick auf die scheinbar gut kontrollierbaren digitalen Umgebungen wäre hier mehr zu erwarten, beispielsweise was Interventionen für den Kompetenzaufbau von Lehrenden betrifft. Denkbar ist auch anhand von Untersuchungen zu Technologie-Trends Aussagen über Anforderungen an Lehrende zu erhalten, insbesondere in Bezug auf die Gestaltung von Lehr-LernArrangements (z.B. neuartige Tools, Verhältnis Lehrende/Lernende, Lernformen). Dies wäre ein sinnvoller Beitrag zur Ausarbeitung von Kompetenzmodellen und der Entwicklung angepasster Aus- und Weiterbildungsangebote für Lehrende. Digitale Technologien, die als relevant für die Weiterentwicklung im Bildungsbereich identifiziert wurden, könnten im Rahmen von Interventionsstudien auf ihre Relevanz für Lehrende untersucht werden. Studien, die nach den Gelingensbedingungen für die Implementation von Kompetenzstandards oder effektiven und nachhaltigen Fortbildungsstrukturen fragen, fehlen ebenso. Hier öffnet sich folglich ein weites Feld für systematische, an Kompetenzstandards orientierte Interventions- und Implementationsstudien, um Erkenntnisse zu erlangen, welche Formen der Aus- und Fortbildung von Lehrenden effektiv sind und wie diese erfolgreich und nachhaltig durch Institutionen oder überinstitutionelle Akteure der Erwachsenenbildung und Weiterbildung etabliert werden können.

Auffällig ist, dass eine grundsätzliche kritische Auseinandersetzung mit der Digitalisierung und ihren Effekten auf Lehrende und die Gestaltung von Bildungsangeboten in den hier betrachteten Publikationen kaum stattfindet. Digitalisierung ist kein neutraler Begriff, sondern ist im gesellschaftlichen bzw. politischen Diskurs mit Setzungen aufgeladen. Beispielsweise werden beim Erwerb digitaler Kompetenzen klassische Bildungsideale mit wirtschaftsorientierten Zielsetzungen wie z.B. Beschäftigungsfähigkeit unreflektiert auf eine Stufe gestellt (vgl. Dander, 2018, S. 264f.). Digitale Entwicklungen werden in den Studien mit ihren Chancen und Herausforderungen wohl wahrgenommen, in ihrer Sinnhaftigkeit oder Unvermeidbarkeit aber nicht in Frage gestellt, wie das folgende Beispiel zeigt: 
„[D] evelopments in information and communication technologies (ICT) are changing the nature of work. This leads in turn to curriculum changes, and thus [...] teachers must be prepared to change" (Colliander, 2019, S. 55 f.). Lediglich Rohs und Weber (2018, S. $12 \mathrm{ff}$.) adressieren in ihrer Bestandsaufnahme zum Thema digitale Medien in der wissenschaftlichen Weiterbildung auch Risiken, dabei stehen Lehrende jedoch nicht im Fokus. In anderen Bildungsbereichen wie etwa Schule oder frühe Bildung sind allgemein Risiken und negative Aspekte von digitalen Medien und der Digitalisierung ein prominenter Teil des Diskurses (vgl. z. B. Fröhlich-Gildhoff \& Fröhlich-Gildhoff, 2017; Schaumburg, 2015).

\subsection{Implikationen für Praxis und Politik}

Bei der Recherche für dieses Review standen die Lehrenden im Fokus. Die methodische Vielfalt und die hohe Zahl von Studien mit Einzelfallcharakter erschweren die Rezeption durch Akteure der Praxis. Dennoch lassen sich Implikationen destillieren. Lehrende sind entscheidend für die Entwicklung und Durchführung erfolgreicher digitaler Angebote. Dazu benötigen sie Unterstützung, um den notwendigen Rollenwechsel $\mathrm{zu}$ vollziehen, eine adäquate Haltung zu entwickeln und erforderliche Kompetenzen aufzubauen. Hier sind passgenaue Fortbildungen nötig, die auf vorliegende Kompetenzmodelle aufbauen (z. B. Redecker \& Punie, 2017; Rohs, Rott et al., 2017; Strauch et al., 2019). Zudem müssen Lehrende zusammenarbeiten, um die vielfältigen Anforderungen digitaler Bildungsangebote bewältigen zu können. Hierzu eignen sich auch ggf. feste Teams (Ferguson et al., 2018). Diese böten nebenbei Gelegenheiten zum flankierenden informellen Peer-Lernen, das mit der bevorzugten Aneignungsstrategie für digitale Kompetenzen bei Lehrenden korrespondiert. Vor diesem Hintergrund ist die bestehende Beschäftigungsstruktur der Lehrenden vermutlich ein nicht unbeträchtliches Hindernis, wenn es darum geht entsprechende Gemeinschaften $\mathrm{zu}$ initiieren und $\mathrm{zu}$ fördern. Hier sind sowohl die Bildungseinrichtungen gefragt, entsprechende Möglichkeiten und Anreize zu bieten und als auch die Politik, entsprechende Anstrengungen substanziell zu fördern.

Die vorliegende Evidenz bestätigt, dass die Digitalisierung hohe Anforderungen an Lehrende stellt. Die angesprochenen Trendstudien (z. B. Adams Becker et al., 2018; Adams Becker et al., 2017; mmb Trendmonitor 2017/2018; mmb Trendmonitor 2018/2019) spiegeln eine große Dynamik im Sektor digitaler Bildungstechnologien wider, auf die Lehrende reagieren müssen, indem sie ihre Kompetenzen stetig erweitern bzw. anpassen. Auf europäischer Ebene ist die Notwendigkeit des Aufbaus digitaler Kompetenzen von Lehrenden im bildungspolitischen Diskurs angekommen (vgl. Pachner, 2018). Sie mündet in konkrete praxisrelevante Entwicklungen, z. B. in das DigCompEdu-Kompetenzmodell (Redecker \& Punie, 2017). Das Modell wird von der Praxis bereits aufgegriffen (vgl. DVV, 2019, S. 4). Auch die Unterstützung der Lehrenden und ihre Qualifizierung ist auf dieser Ebene als 
Notwendigkeit anerkannt (vgl. Europäische Kommission, 2018, S. 6). Das Projekt COMPALL kann in diesem Zusammenhang verortet werden. Es wurde vom europäischen ERASMUS+-Programm gefördert und setzte erfolgreich digitale Medien ein, um die Internationalisierung der akademischen Ausbildung in der EB/WB zu unterstützen. Auch im Diskurs in Deutschland ist die Bedeutung der Lehrenden und die Dringlichkeit ihrer Qualifikation anerkannt (vgl. BMBF, 2019; KMK, 2017, S. $56 \mathrm{f}$.). Wie oben ausgeführt fehlt es in der EB/WB jedoch derzeit noch an grundlegender wissenschaftlicher Evidenz für bildungspolitische Steuerungsmaßnahmen, beispielsweise zum Bestand und dem Niveau von digitalen Kompetenzen bei Lehrenden. Lohnend können Projekte sein, die entsprechende Evidenz produzieren oder Möglichkeiten und Bedingungen für die Implementation effektiver und nachhaltiger Maßnahmen für Lehrende in Zusammenarbeit mit der Praxis erkunden. Im Zuge aktuellster Entwicklungen in Verbindung mit der Corona-Pandemie und der damit einhergehenden Weisung strikter Kontaktvermeidung ist ein regelrechter Zusammenbruch der Präsenzlehre zu beobachten. Die Umsetzung digitaler Veranstaltungsformate ist für viele Lehrende innerhalb kürzester Zeit existenziell bedeutsam geworden. Es ist zu erwarten, dass diese Umwälzung auch nach dem Abebben der Pandemie die Rahmenbedingungen für die Nutzung digitaler Medien in der Bildung verändern wird. In Anbetracht der großen Forschungslücken sind weitere Untersuchungen daher zukünftig nicht nur unabdingbar, sondern dringender denn je.

\section{Literatur}

* durch Reviewprozess ausgewählte Studie

*Adams Becker, S., Brown, M., Dahlstrom, E., Davis, A., DePaul, K., Diaz, V. et al. (2018). NMC Horizon Report: 2018 Higher Education Edition. Louisville: EDUCAUSE.

*Adams Becker, S., Cummins, M., Davis, A., Freeman, A., Hall Giesinger, C. \& Ananthanarayanan, V. (2017). NMC Horizon Report. 2017 Higher Education Edition. Austin: New Media Consortium Verfügbar unter ERIC - ED582134 - NMC Horizon Report: 2017 Higher Education Edition, New Media Consortium, 2017.

*Alexander, B., Ashford-Rowe, K., Barajas-Murphy, N., Dobbin, G., Knott, J., McCormack, M., Pomerantz, J., Seilhamer, R. \& Weber, N. (2019). EDUCAUSE Horizon Report. 2019 Higher Education Edition. Louisville: EDUCAUSE.

${ }^{*}$ Arnold, R., Nuissl, E. \& Rohs, M. (2017). Erwachsenenbildung. Eine Einführung in Grundlagen, Probleme und Perspektiven. Baltmannsweiler: Schneider Hohengehren.

*Autorengruppe wb-personalmonitor. (2016). Das Personal in der Weiterbildung. Arbeitsund Beschäftigungsbedingungen, Qualifikationen, Einstellungen zu Arbeit und Beruf. Bielefeld: W. Bertelsmann Verlag. https://doi.org/10.3278/85/0015w

$\mathrm{BMBF}=$ Bundesministerium für Bildung und Forschung. (2019). Strategiepapier Nationale Weiterbildungsstrategie. o.O. Verfügbar unter https://www.bmbf.de/files/NWS_Strategie papier_barrierefrei_DE.pdf 
Boeren, E. (2017). The Methodological Underdog. A Review of Quantitative Research in the Key Adult Education Journals. Adult Education Quarterly: A Journal of Research and Theory, 68(1), 63-79. https://doi.org/10.1177/0741713617739347

*Bolten, R. (2018). Der mediale Habitus als System von Grenzen medienpädagogischen Handelns von Lehrenden in der Erwachsenenbildung. MedienPädagogik: Zeitschrift für Theorie und Praxis der Medienbildung, (32), 96-107. https://doi.org/10.21240/ MPAED/32/2018.10.26.X

*Bolten, R., Kohl, J. \& Rohs, M. (2018). Digitale Infrastruktur der Volkshochschulen in Rheinland-Pfalz (Beiträge zur Erwachsenenbildung). Verfügbar unter http://nbn-resolv ing.de/urn:nbn:de:hbz:386-kluedo-52376

${ }^{\star}$ Bolten, R., Rohs, M., Schmidt-Hertha, B. \& Rott, K. J. (2019). Measurement of media pedagogical competences of adult educators. European journal for Research on the Education and Learning of Adults, 10(3), 307-324. https://doi.org/10.3384/rela.2000-7426.ojs393

*Bolten, R. \& Rott, K. J. (2018). Medienpädagogische Kompetenz. Anforderungen an Lehrende in der Erwachsenenbildung. Perspektiven der Praxis. MedienPädagogik: Zeitschrift für Theorie und Praxis der Medienbildung, Heft 30: Medienpädagogik und Erwachsenenbildung, 137-153. https://doi.org/10.21240/MPAED/30/2018.03.05.X

Booth, A., Sutton, A. \& Papaioannou, D. (2016). Systematic approaches to a successful literature review (2. Auflage). Los Angeles: Sage.

Bosche, B., Schneider, M. \& Strauch, A. (2018). Lehrkompetenzen in der Erwachsenen- und Weiterbildung. Bilanzierung und Möglichkeiten der Anerkennung mit dem Instrument PortfolioPlus. Berufsbildung in Wissenschaft und Praxis, 47(1), 47-50.

${ }^{*}$ Bremer, C. (2017). Mobiles Lernen in der Erwachsenenbildung. Optionen für Lernszenarien. In F. Thissen (Hrsg.), Lernen in virtuellen Räumen. Perspektiven des mobilen Lernens (Lernwelten, S. 88-109). Berlin: De Gruyter Saur. https://doi.org/10. 1515/9783110501131-007

${ }^{\star}$ Burchert, J. \& Grobe, R. (2017). Herausforderungen bei der Implementierung digital gestützter beruflicher Weiterbildung. Die Sicht von WeiterbildnerInnen und BildungsmanagerInnen auf Strukturen kulturelle Praktiken und Agency. Magazin Erwachsenenbildung.at, 11(30). Verfügbar unter http://nbn-resolving.de/urn:nbn: de:0111-pedocs128852

${ }^{\star}$ Caena, F. \& Redecker, C. (2019). Aligning teacher competence frameworks to 21st century challenges. The case for the European Digital Competence Framework for Educators (Digcompedu). European Journal of Education, 14, 317. https://doi.org/10.1111/ ejed.12345

${ }^{\star}$ Cendon, E. (2018). Lifelong Learning at Universities. Future Perspectives for Teaching and Learning. Journal of New Approaches in Educational Research, 7(2), 81-87. https://eric. ed.gov/?id=EJ1185400. https://doi.org/10.7821/naer.2018.7.320

${ }^{*}$ Colliander, H. (2019). Being Transformed and Transforming Oneself in a Time of Change. A Study of Teacher Identity in Second Language Education for Adults. Studies in the Education of Adults, 51(1), 55-73. https://doi.org/10.1080/02660830.2018.1526447

Dander, V. (2018). Ideologische Aspekte von „Digitalisierung“. Eine Kritik des bildungspolitischen Diskurses um das KMK-Strategiepapier „Bildung in der digitalen Welt“. In C. Leineweber \& C. de Witt (Hrsg.), Digitale Transformation im Diskurs. Kritische Perspektiven auf Entwicklungen und Tendenzen im Zeitalter des Digitalen (S. 252-279). Online-Sammelband.

Diethelm, I. (2018). Digitalisierung und Schule: zwischen Buzzword-Bingo und Allgemeinbildungsauftrag. Zeitschrift für Bildungsverwaltung, 34(2), 37-46. Verfügbar unter https://www.dgbv.de/mediapool/118/1188921/data/2018/ZBV-Heft-2018-2.pdf 
Dollhausen, K. (Hrsg.). (2008). Planungskulturen in der Weiterbildung. Angebotsplanungen zwischen wirtschaftlichen Erfordernissen und pädagogischem Anspruch (Theorie und Praxis der Erwachsenenbildung). Bielefeld: Bertelsmann. Verfügbar unter http://deposit.d-nb.de/cgi-bin/dokserv?id=3105424\&prov=M\&dok_var=1\&dok_ext=htm

DVV = Deutscher Volkshochschulverband. (2019). Manifest zur digitalen Transformation von Volkshochschulen. o. O., o. V. Verfügbar unter https://www.volkshochschule.de/ medien/downloads/verbandswelt/digitalisierungsstrategie/Manifest_Digitale_Transfor mation.pdf

Europäische Kommission. (2018). Aktionsplan für digitale Bildung. Brüssel: Eigenverlag. Verfügbar unter https://eur-lex.europa.eu/legal-content/DE/TXT/PDF/?uri=CELEX: 52018DC0022\&from $=$ EN

Faulstich, P., Zeuner, C. (1999). Erwachsenenbildung. Eine handlungsorientierte Einführung in Theorie, Didaktik und Adressaten. Weinheim und München: Beltz.

${ }^{\star}$ Ferguson, R., Herodotou, C., Coughlan, T., Scanlon, E. \& Sharples, M. (2018). MOOC Development. In R. Luckin (Hrsg.), Enhancing Learning and Teaching with Technology. What the Research Says (S. 205-213). London: UCL IOE Press. https://www.cul.ioe. press.com

Fontana, R. P., Milligan, C., Littlejohn, A. \& Margaryan, A. (2015). Measuring self-regulated learning in the workplace. International Journal of Training and Development, 19(1), 32-52. https://doi.org/10.1111/ijtd.12046

Fröhlich-Gildhoff, K. \& Fröhlich-Gildhoff, M. (2017). Digitale Medien in der Kita die Risiken werden unterschätzt! Frühe Bildung, 6(4), 225-228. https://doi.org/10. 1026/2191-9186/a000332

${ }^{\star}$ Ghomi, M. \& Redecker, C. (2019). Digital Competence of Educators (DigCompEdu). Development and Evaluation of a Self-assessment Instrument for Teachers' Digital Competence. In Proceedings of the 11th International Conference on Computer Supported Education (S. 541-548). SCITEPRESS - Science and Technology Publications. https://doi.org/10.5220/0007679005410548

Grant, M. J. \& Booth, A. (2009). A typology of reviews. An analysis of 14 review types and associated methodologies. Health information and libraries journal, 26(2), 91-108. https://doi.org/10.1111/j.1471-1842.2009.00848.x

${ }^{\star}$ Helbig, C. \& Hofhues, S. (2018). Leitideen in der medienpädagogischen Erwachsenenbildung. Ein analytischer Blick. MedienPädagogik: Zeitschrift für Theorie und Praxis der Medienbildung, Heft 30: Medienpädagogik und Erwachsenenbildung, (30), 1-17. https:// doi.org/10.21240/MPAED/30/2018.02.26.X

Herring, M. C., Koehler, M. J. \& Mishra, P. (2016). Handbook of technological pedagogical content knowledge (TPACK) for educators (2. Auflage). New York: Routledge. https:// doi.org/10.4324/9781315771328

Johnson, L., Adams Becker, S., Cummins, M., Estrada, V., Freeman, A. \& Hall, C. (2016). NMC Horizon Report: 2016 Higher Education Edition: Deutsche Ausgabe. Austin: The New Media Consortium. Verfügbar unter https://www.mmkh.de/fileadmin/dokumen te/Publikationen/2016-nmc-horizon-report-he-DE.pdf

Johnson, L., Adams Becker, S., Cummins, M., Estrada, V., Freeman, A. \& Ludgate, H. (2013). NMC Horizon Report: 2013 Higher Education Edition: Deutsche Ausgabe. Austin: The New Media Consortium. Verfügbar unter https://www.mmkh.de/filead $\mathrm{min} /$ dokumente/Publikationen/2013-horizon-report-HE-German.pdf

${ }^{\star}$ Kennedy, I. G., Latham, G. \& Jacinto, H. (2016). Education skills for 21st century teachers. Voices from a global online educators' forum (Springer briefs in education). Cham: Springer. https://doi.org/10.1007/978-3-319-22608-8 
${ }^{*}$ Klauser, F. \& Schlicht, J. (2016). Vernetzung - Kernprozess und Resultat technologiegestützter Bildung, Gestaltungsaufgaben für Teletutoren - Best Practice. In M. Schulz, B. Griebenow, A. Neusius, C. Vogeler \& K. Papenberg (Hrsg.), Fernausbildung schärft Perspektiven ... Technologiegestützte Bildung als Motor von Innovationsprozessen (Grundlagen der Weiterbildung, S. 170-185). Augsburg: Ziel-Verl.

KMK = Ständige Konferenz der Kultusminister der Länder in der Bundesrepublik Deutschland. (2017). Bildung in der digitalen Welt. Strategie der Kultusministerkonferenz. Beschluss der Kultusministerkonferenz vom 08.12.2016 in der Fassung vom 07.12.2017. Berlin: Eigenverlag. Verfügbar unter https://www.kmk.org/fileadmin/ Dateien/pdf/PresseUndAktuelles/2018/Digitalstrategie_2017_mit_Weiterbildung.pdf

Kraft, S. (2018). Berufsfeld Weiterbildung. In R. Tippelt \& A. v. Hippel (Hrsg.), Handbuch Erwachsenenbildung/Weiterbildung. Band 2. (6., überarb. u. aktual. Aufl., Springer Reference Sozialwissenschaften, S. 1109-1128). Wiesbaden: Springer VS. https://doi. org/10.1007/978-3-531-19979-5_51

${ }^{\star}$ Kühn, C., Robak, S. \& Fleige, M. (2018). Programmplanung in einer Kultur der Digitalität. Verbundprojekt der Universität Hannover und des Deutschen Instituts für Erwachsenenbildung. Erwachsenenbildung (KEB), 64(4), S. 184-186.

${ }^{\star}$ Laurian-Fitzgerald, S., Fitzgerald, C. J., Popa, C. A. \& Bochis, L. (2018). The New Normal: Adult Online Learners. In C. J. Fitzgerald, S. Laurian-Fitzgerald \& C. Popa (Hrsg.), Handbook of Research on Student-Centered Strategies in Online Adult Learning Environments (Advances in Educational Technologies and Instructional Design (AETID) Book Series, S. 140-171). https://doi.org/10.4018/978-1-5225-5085-3.ch007

${ }^{\star}$ Littlejohn, A. \& Hood, N. (2016). How Educators Build Knowledge and Expand Their Practice. The Case of Open Education Resources. British Journal of Educational Technology, 48(2), 499-510. https://doi.org/10.1111/bjet.12438

${ }^{\star}$ Loizzo, J., Ertmer, P. A., Watson, W. R. \& Watson, S. L. (2017). Adult MOOC Learners as Self-Directed. Perceptions of Motivation, Success, and Completion. Online Learning, 21(2), 24 S. Verfügbar unter https://files.eric.ed.gov/fulltext/EJ1149353.pdf. https://doi. org/10.24059/olj.v21i2.889

Marx, C., Goeze, A., Kelava, A. \& Schrader, J. (2018). Lehrkräfte in der Erwachsenenund Weiterbildung - Zusammenhänge zwischen Vorbildung und Erfahrung mit dem Wissen über Lehr-Lernmethoden und -konzepte. Zeitschrift für Weiterbildungsforschung, 41(1), 57-77. https://doi.org/10.1007/s40955-018-0108-6

${ }^{*}$ Mavropoulos, A. A., Sipitanou, A. K. \& Pampouri, A. A. (2019). „Training of Adult Trainers". Implementation and Evaluation of a Higher Education Program in Greece. International Review of Research in Open and Distributed Learning, 20(1), 279-288. https://doi.org/10.19173/irrodl.v20i1.4143

*Mayert, A. (2018). Digitalisierung in der öffentlich geförderten Erwachsenenbildung. Große Erwartungen, wenig Unterstützung, dennoch Chancen. Forum Erwachsenenbildung, (3), 14-18. https://www.waxmann.com/waxmann-zeitschriften/waxmann-zeitschriftendetails/?no_cache=1\&tx_p2waxmann_pi2[zeitschrift]=ZEI1028\&tx_p2waxmann_ pi2[ausgabe]=AUG100292\&tx_p2waxmann_pi2[action]=ausgabe\&tx_p2waxmann_ pi2[controller]=Zeitschrift\&cHash=0d94112076d8fb00edafc16629fd0eea

${ }^{\star}$ Mayrberger, K. (2017). Miteinander lernen auf Augenhöhe. Partizipatives Lernen unter den Bedingungen der Digitalisierung gestalten. Weiterbildung, (4), 32-35.

${ }^{*}$ mmb Institut (Hrsg.). (2016). Digitale Bildung auf dem Weg ins Jahr 2025. Schlussbericht zur Trendstudie. Essen: mmb Institut. Verfügbar unter https://www.mastersolution.de/ files/nachrichten/blog/2017/studie-zur-digitalen-bildung-learntec-2017.pdf 
${ }^{\star}$ mmb-Trendmonitor I/2017. (2017). Corporate Learning wird zum Cyber-Learning. Ergebnisse der 11. Trendstudie „mmb Learning Delphi“. Essen: mmb Institut. Verfügbar unter https://mmb-institut.de/wp-content/uploads/mmb-Trendmonitor_2016-2017.pdf

*mmb-Trendmonitor 2017/2018. (2018). Erklärfilme als Umsatzbringer der Stunde. Ergebnisse der 12. Trendstudie „mmb Learning Delphi“. Essen: mmb Institut. Verfügbar unter https://www.mmb-institut.de/wp-content/uploads/mmb-Trendmonitor_2017-2018.pdf

${ }^{*}$ mmb-Trendmonitor 2018/2019. (2018). Auf dem Weg zum Assisted Learning? Digitale Lernanwendungen werden informeller und intelligenter. Ergebnisse der 13. Trendstudie „mmb Learning Delphi“. Essen: mmb Institut. Verfügbar unter https://www.mmb-insti tut.de/wp-content/uploads/mmb-Trendmonitor_2018-2019.pdf

Mochmann, E. (2019). Quantitative Daten für die Sekundärananlyse. In N. Baur \& J. Blasius (Hrsg.), Handbuch Methoden der empirischen Sozialforschung (2. Auflage, S. 259-270). Wiesbaden: Springer VS. https://doi.org/10.1007/978-3-658-21308-4_17

${ }^{\star}$ Montebello, M. Digital Pedagogies for Teachers; CPD [Konferenzbericht] Paper presented at the International Association for Development of the Information Society (IADIS) International Conference on Educational Technologies (5th, Sydney, Australia, Dec 11-13, 2017). Verfügbar unter https://eric.ed.gov/?id=ED579307

*Nafukho, F. M., Alfred, M., Chakraborty, M., Johnson, M. \& Cherrstrom, C. A. (2017). Predicting Workplace Transfer of Learning. A Study of Adult Learners Enrolled in a Continuing Professional Education Training Program. European Journal of Training and Development, 41(4), 327-353. https://doi.org/10.1108/EJTD-10-2016-0079

${ }^{*}$ Niedermeier, S. \& Müller, C. (2016). Game-Based-Learning in Aus- und Weiterbildung - von der Idee zur Umsetzung. In J. Wachtler \& M. Ebner (Hrsg.), Digitale Medien: Zusammenarbeit in der Bildung (Medien in der Wissenschaft. 71, S. 190-200). Münster: Waxmann. Verfügbar unter http://nbn-resolving.org/urn:nbn:de:0111-pedocs-157927

Nuissl von Rein, E. (2012). Didaktik und lernende Erwachsene. In W. Gieseke, E. Nuissl von Rein \& I. Schüßler (Hrsg.), Reflexion zur Selbstbildung. Festschrift für Rolf Arnold (S. 83-101). Bielefeld: W. Bertelsmann.

*Pachner, A. (2018). Digital unterstützte Lernprozesse - Chancen und Herausforderungen für die Rolle der Lernenden. Forum Erwachsenenbildung, (3), 19-23. Verfügbar unter https://www.waxmann.com/waxmann-zeitschriftendetails/?no_cache=1\&tx_p2wax mann_pi2[zeitschrift]=ZEI1028\&tx_p2waxmann_pi2 [ausgabe]=AUG100292\&tx_ p2waxmann_pi2[action]=ausgabe\&tx_p2waxmann_pi2[controller]=Zeitschrift\&cHash $=0 \mathrm{~d} 94112076 \mathrm{~d} 8 \mathrm{fb} 00 \mathrm{edafc} 16629 \mathrm{fd} 0 \mathrm{eea}$

*Paepe, L. de, Zhu, C. \& Depryck, K. (2018). Online Dutch L2 Learning in Adult Education. Educators' and Providers' Viewpoints on Needs, Advantages and Disadvantages. Open Learning, 33(1), 18-33. https://doi.org/10.1080/02680513.2017.1414586

${ }^{\star}$ Paepe, L. de, Zhu, C. \& Depryck, K. (2019). Development and implementation of online Dutch L2 courses in adult education. Educators' and providers' perceptions of constraints and critical success factors. Innovation in Language Learning and Teaching, 22(13, 3), 277-291. https://doi.org/10.1080/17501229.2018.1462369

*Pérez-Paredes, P., Ordoñana Guillamón, C. \& Aguado Jiménez, P. (2018). Language Teachers' Perceptions on the Use of OER Language Processing Technologies in MALL. Computer Assisted Language Learning, 31, 522-545. https://doi.org/10.1080/09588221. 2017.1418754

${ }^{\star}$ Redecker, C. \& Punie, Y. (2017). European framework for the digital competence of educators. DigCompEdu (EUR, Scientific and technical research series, Bd. 28775). Luxembourg: Publications Office.

${ }^{\star}$ Richter, T. (2017). Betriebliche Weiterbildung als Antwort auf die Implementierung von Industrie 4.0. In G. Spöttl \& L. Windelband (Hrsg.), Industrie 4.0. Risiken und Chancen 
für die Berufsbildung (Berufsbildung, Arbeit und Innovation. 44, S. 241-260). Bielefeld: Bertelsmann.

${ }^{\star}$ Roberts, J. (2018). Future and Changing Roles of Staff in Distance Education. A Study to Identify Training and Professional Development Needs. Distance Education, 39(1), 3753. https://doi.org/10.1080/01587919.2017.1419818

${ }^{\star}$ Rohs, M. \& Bolten, R. (2017). Medienpädagogische Professionalisierung von Erwachsenenbildner*innen. Eine biographische Annäherung (Beiträge zur Erwachsenenbildung. 4). Kaiserslautern: Techn. Univ. Verfügbar unter http://nbn-resolving.de/urn:nbn:de: hbz:386-kluedo-45475

${ }^{\star}$ Rohs, M., Bolten, R. \& Kohl, J. (2017). Medienpädagogische Kompetenzen in Kompetenzbeschreibungen für Erwachsenenbildner* innen. Beiträge zur Erwachsenenbildung Nr. 5). Kaiserslautern: Techn. Univ. Verfügbar unter https://nbn-resolving.org/urn:nbn:de: hbz:386-kluedo-46905

${ }^{\star}$ Rohs, M., Rott, K. J., Schmidt-Hertha, B. \& Bolten, R. (2017). Medienpädagogische Kompetenzen von ErwachsenenbildnerInnen. Magazin Erwachsenenbildung.at, 11(30). Verfügbar unter http://nbn-resolving.de/urn:nbn:de:0111-pedocs-128873

${ }^{\star}$ Rohs, M. \& Weber, C. (2018). Digitale Medien in der wissenschaftlichen Weiterbildung. Handbuch Wissenschaftliche Weiterbildung, 1-25. https://doi.org/10.1007/978-3-65817674-7_23-1

${ }^{\star}$ Rossmann, E. D. (2016). Nur Weiterbildung kann die digitale Spaltung verhindern. Der Nationale IT-Gipfel, die digitale Bildungsstrategie der Bundesregierung und die Potenziale der Erwachsenenbildung. Dis.kurs, (4), 4-7.

${ }^{\star}$ Rott, K. J. \& Schmidt-Hertha, B. (2019). Medienpädagogische Kompetenz von erwachsenenpädagogischen Fachkräften - Eine Frage des Alters? In E. Haberzeth \& I. Sgier (Hrsg.), Digitalisierung und Lernen. Gestaltungsperspektiven für das professionelle Handeln in der Erwachsenenbildung und Weiterbildung (Forum Hochschuldidaktik und Erwachsenenbildung, Band 8, S. 137-154). Bern: hep.

Schaumburg, H. (2015). Chancen und Risiken digitaler Medien in der Schule. In Bertelsmann Stiftung (Hrsg.), Individuell fördern mit digitalen Medien. Chancen, Risiken, Erfolgsfaktoren (S. 20-94). Bielefeld: Verlag Bertelsmann Stiftung.

${ }^{*}$ Schmid, U., Goertz, L. \& Behrens, J. (Hrsg.). (2017). Die Weiterbildung im digitalen Zeitalter (Monitor Digitale Bildung. 04). Gütersloh. https://doi.org/10.11586/2018007

${ }^{\star}$ Schöb, S., Rohs, M., Biel, C. \& Scholze, T. (2016). Professionalisierung von Lehrhandeln in einer digital geprägten Lernkultur. Bedarf und Ansatzpunkte der Entwicklung einer Online-Lernumgebung. In O. Dörner, C. Iller, H. Pätzold \& S. Robak (Hrsg.), Differente Lernkulturen - regional, national, transnational (Schriftenreihe der Sektion Erwachsenenbildung in der Deutschen Gesellschaft für Erziehungswissenschaft (DGfE), S. 175-187). Opladen u. a.: Budrich. https://doi.org/10.2307/j.ctvbkk1kt.16

Schöll, I. (2017). (Keine) Zeit für Experimente. Beobachtungen zur Digitalisierung der Volkshochschulen. DIE-Zeitschrift für Erwachsenenbildung, (3), 32-34. https://doi. org/10.3278/DIE1703W032

Schrader, J. (2010). Fortbildung von Lehrenden der Erwachsenenbildung: Notwendig? Sinnvoll? Möglich? In J. Schrader, R. Hohmann \& S. Hartz (Hrsg.), Mediengestützte Fallarbeit: Konzepte, Erfahrungen und Befunde zur Kompetenzentwicklung von Erwachsenenbildnern (S. 25-68). Bielefeld: W. Bertelsmann.

Schrader, J. (2011). Struktur und Wandel der Weiterbildung. Bielefeld: Bertelsmann W. Verfügbar unter http://www.content-select.com/index.php?id=bib_view\&ean=9783763 948475

Schrader, J. (2019). Institutionelle Rahmenbedingungen, Anbieter, Angebote und LehrLernprozesse der Erwachsenen- und Weiterbildung. In O. Köller, M. Hasselhorn, F. W. 
Hesse, K. Maaz, J. Schrader, H. Solga et al. (Hrsg.), Das Bildungswesen in Deutschland. Bestand und Potenziale (S. 701-729). Stuttgart: utb.

Schrader, J. \& Loreit, F. (2018). Was ist Erwachsenenbildung? Geschichte, Hintergrund, Ziele. Deutscher Kulturrat. Verfügbar unter https://www.kulturrat.de/themen/kulturelle-bil dung/kulturelle-erwachsenenbildung/was-ist-erwachsenenbildung/

${ }^{*}$ Schrader, J. \& Schöb, S. (2016). Die Planung von Lehr-Lern-Einheiten mit digitalen Medien. Konzepte und Befunde. Zeitschrift für Weiterbildungsforschung - Report, (3), 331-347. https://doi.org/10.1007/s40955-016-0078-5

Schwer, C. \& Solzbacher, C. (Hrsg.). (2014). Professionelle pädagogische Haltung. Historische, theoretische und empirische Zugänge zu einem viel strapazierten Begriff. Bad Heilbrunn: Klinkhardt.

${ }^{*}$ Sgier, I., Haberzeth, E. \& Schüepp, P. (2018). Digitalisierung in der Weiterbildung. Ergebnisse der jährlichen Umfrage bei Weiterbildungsanbietern. Zürich: SVEB, PHZH. Verfügbar unter http://edudoc.ch/record/130478/files/SVEB_Weiterbildungsstudie 2017_2018.pdf

*Staab, M. \& Egetenmeyer, R. (2019). Mit Blended Learning zur Internationalisierung der Hochschulbildung - Die Erasmus+ Strategische Partnerschaft COMPALL. In H. Angenent, B. Heidkamp \& D. Kergel (Hrsg.), Digital Diversity: Bildung und Lernen im Kontext gesellschaftlicher Transformationen (S. 279-290). Wiesbaden: Springer Fachmedien Wiesbaden. https://doi.org/10.1007/978-3-658-26753-7_17

*Staudacher, N. (2019). Digitale Spiele und ihr Potenzial als Bildungs- und Lernräume. Paralleltitel: Digital games and their potential as spaces for education and learning. Magazin Erwachsenenbildung.at, 13, 35-36. Verfügbar unter http://nbn-resolving.org/ urn:nbn:de:0111-pedocs-166730

${ }^{*}$ Strauch, A. \& Lencer, S. (2016). Das GRETA-Kompetenzmodell für Lehrende in der Erwachsenen- und Weiterbildung. (texte.online). Verfügbar unter https://www.diebonn.de/doks/2016-erwachsenenbildung-02.pdf

*Strauch, A., Lencer, S., Bosche, B., Gladkova, V., Schneider, M. \& Treviño-Eberhard, D. (2019). GRETA - kompetent handeln in Training, Kurs \& Seminar. Das GRETA-Kompetenzmodell (texte.online). Verfügbar unter https://www.die-bonn.de/docs/Handrei chung_KM_2019.pdf

${ }^{*}$ Tan, S. C. (2018). Technologies for adult and lifelong education. In M. Milana, S. Webb, J. Holford, R. Waller \& P. Jarvis (Hrsg.), The Palgrave International Handbook on Adult and Lifelong Education and Learning (S. 917-937). London: Palgrave Macmillan UK. https://doi.org/10.1057/978-1-137-55783-4

${ }^{\star}$ Umbach, S., Böving, H. \& Haberzeth, E. (2018). Kompetenzverschiebungen und menschliche Handlungsqualitäten im Digitalisierungsprozess. Ergebnisse aus Betriebsfallstudien in Einzelhandel und Logistik und Konsequenzen. Berufsbildung in Wissenschaft und Praxis, 47(1), 16-19. Verfügbar unter https://www.bibb.de/veroeffentlichungen/de/ bwp/show $/ 8560$

*Wiemeyer, J. (2016). Spielerisches und entdeckendes Lernen - neue Potenziale für die Bildung? In M. Schulz, B. Griebenow, A. Neusius, C. Vogeler \& K. Papenberg (Hrsg.), Fernausbildung schärft Perspektiven ... Technologiegestützte Bildung als Motor von Innovationsprozessen (Grundlagen der Weiterbildung, S. 409-424). Augsburg: Ziel-Verl.

${ }^{\star}$ Wienberg, J., Heinrichs, H. S. \& Grotlüschen, A. (2018). Ideen, Standards und Stolpersteine kollaborativer E-Learning-Projekte. Forum Erwachsenenbildung, (3), 33-37. Verfügbar unter https://www.waxmann.com/waxmann-zeitschriften/waxmann-zeitschriftende tails/?no_cache=1\&tx_p2waxmann_pi2[zeitschrift]=ZEI1028\&tx_p2waxmann_pi2[aus gabe]=AUG100292\&tx_p2waxmann_pi2[action]=ausgabe\&tx_p2waxmann_pi2[con troller] $=$ Zeitschrift \&cHash=0d94112076d8fb00edafc16629fd0eea 
${ }^{*}$ Wildemeersch, D. \& Jütte, W. (2017). Editorial. Digital the new normal - multiple challenges for the education and learning of adults. European Journal for Research on the Education and Learning of Adults, 8(1), 7-20. https://doi.org/10.3384/rela.2000-7426. relae13

Willcox, K. E., Sarma, S. \& Lippel, P. (2016). Online Education: A Catalyst for Higher Education Reforms. Final Report of the MIT Online Education Policy Initiative. Cambridge (MA): MIT. Verfügbar unter https://dspace.mit.edu/bitstream/handle/ 1721.1/103912/MIT\%20Online\%20Education\%20Policy\%20Initiative\%20April\%20 2016_0.pdf?sequence $=1$ \&isAllowed $=y$ 
